\title{
Characterization of skeletal muscle performance and morphology following acute and chronic mechanical loading paradigms
}

\author{
Brent A. Baker \\ West Virginia University
}

Follow this and additional works at: https://researchrepository.wvu.edu/etd

\section{Recommended Citation}

Baker, Brent A., "Characterization of skeletal muscle performance and morphology following acute and chronic mechanical loading paradigms" (2007). Graduate Theses, Dissertations, and Problem Reports. 4289.

https://researchrepository.wvu.edu/etd/4289

This Dissertation is protected by copyright and/or related rights. It has been brought to you by the The Research Repository @ WVU with permission from the rights-holder(s). You are free to use this Dissertation in any way that is permitted by the copyright and related rights legislation that applies to your use. For other uses you must obtain permission from the rights-holder(s) directly, unless additional rights are indicated by a Creative Commons license in the record and/ or on the work itself. This Dissertation has been accepted for inclusion in WVU Graduate Theses, Dissertations, and Problem Reports collection by an authorized administrator of The Research Repository @ WVU.

For more information, please contact researchrepository@mail.wvu.edu. 
Characterization of Skeletal Muscle Performance and Morphology Following Acute and Chronic Mechanical Loading Paradigms

Brent A. Baker, MS, ATC.

Dissertation submitted to the School of Medicine at West Virginia University

In partial fulfillment of the requirements

For the degree of

Doctor of Philosophy

In Exercise Physiology

Robert G. Cutlip, Ph.D., Chair

Stephen E. Alway, Ph.D.

Robert R. Mercer, Ph.D.

Michael L. Kashon, Ph.D.

Randall W. Bryner, Ed.D.

Robert D. Chetlin, Ph.D.

Morgantown, West Virginia

2007

Keywords: Aging, Muscle Injury, Muscle Adaptation, Muscle Regeneration, StretchShortening Contractions 


\begin{abstract}
Characterization of Skeletal Muscle Performance and Morphology Following Acute and Chronic Mechanical Loading Paradigms

Brent A. Baker
\end{abstract}

Unaccustomed muscular activity (incorporating lengthening-type movements) results in skeletal muscle damage, yet effects in performance and muscle morphology from movements such as stretch-shortening contractions (which are common-type movements in daily activities) are not well characterized or quantified. Thus, determining time- and dose-dependent responses resulting from acute mechanical exposure will aid critically in understanding muscle injury kinetics and the role of repair and regeneration following this type of exposure. Furthermore, skeletal muscle mass and function declines with age and these events result in a significant impact on the aging workforce. Differentiating the response following both acute and chronic adaptive exposures in young and aged populations will advance our understanding of how the regenerative capacity of skeletal muscle may differ with age. In the absence of overt muscle damage, oxidative stress mismanagement may influence the adaptive/mal-adaptive response following high-intensity mechanical loading. The glutathione system greatly impacts multiple pathways and functions directly as a reducing agent, serving as a substrate for glutathione peroxidase (and other enzymes), and functioning in the recycling of radicals generated from other molecules in skeletal muscle. The adaptive response ensuing from manipulating the glutathione system was ascertained with respect to age following chronic mechanical exposures. Surprisingly, the impact of oxidative stress was negligible, irrespective of age following SSC 
exposure. However, our results suggest initial muscle regeneration is a critical element in assuring successful, long-term adaptation. Remarkably the capacity to respond efficiently to an initial mechanical stimulus as one ages, may be one of the most important factors that ultimately influences adaptation of skeletal muscle. 


\section{Dedications}

Since I have now reached a culminating point in my education, I am left to reflect on those closest to me that have been so loving and so tolerable of me during the pursuit of my dreams and aspirations. To my wife Anna, thank you does not begin to convey my gratitude within me for your love, patience, and support throughout this time in my life. Yet, here I am, finally at this point and I say thank you - I love you always. To my dearest Mia Luciana, in time you will come to understand that you are a shining light, not only to me but your mother too, and during this time you have been my light - I love you baby girl with lots of Eskimo kisses! To my mom, dad, and nan, without your love, endless sacrifices, and constant intercession throughout my life, I would not be were I am - I love you and admire you always. To my brother Bradley, thank you so much for being there just to listen and believing in me - I love you and appreciate you greatly. 


\section{Acknowledgements}

There are several individuals that I would like to extend my thanks to whom have been instrumental in the completion of this dissertation project and throughout my graduate education. My most gracious thanks goes out to my advisor, mentor, and friend Dr. Robert G. Cutlip, whose guidance and belief in my abilities have made an impact on me beyond the scope of this work. Thank you so very much for your patience and constant encouragement, as well as for being a model human being. I have matured immensely as a scientist over the course of our time together, and will continue to carry a positive outlook into all future endeavors because of your efforts. Additionally, I would like to give a special thanks to my committee members, Dr. Stephen Alway, Dr. Robert Mercer, Dr. Michael Kashon, Dr. Randall Bryner, and Dr. Robert Chetlin for continual support and encouragement along this arduous process. For your patience and understanding, for your tolerance in my limitations, and for the time you have taken in your lives to make my educational and professional progression a priority - thank you always. The innumerable individuals that have impacted my graduate education cannot go unnoticed nor can their impact be understated, so at this time I extend my deepest thanks and indebtedness to all for your advice, assistance, and kindness. Finally, the research opportunity afforded to me by working at NIOSH; this will always be valued. I thank all of those individuals who have supported me throughout my time with this agency, especially in the Health Effects Laboratory Division. My employment at NIOSH has allowed me to develop professionally in one of the most constructive and dynamic environments available. 
Table of Contents

Abstract................................................................................ii

Dedications.......................................................................

Acknowledgments...........................................................................v

Table of Contents..................................................................vi

List of Tables and Figures.......................................................

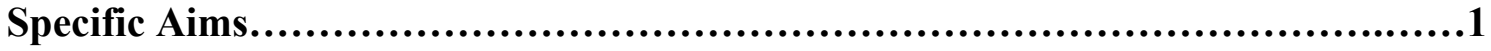

Background and Significance.....................................................

References...............................................................................20

Chapter 1: Stereological Analysis of Muscle Morphology Following Exposure to Repetitive Stretch-Shortening Cycles in a Rat Model

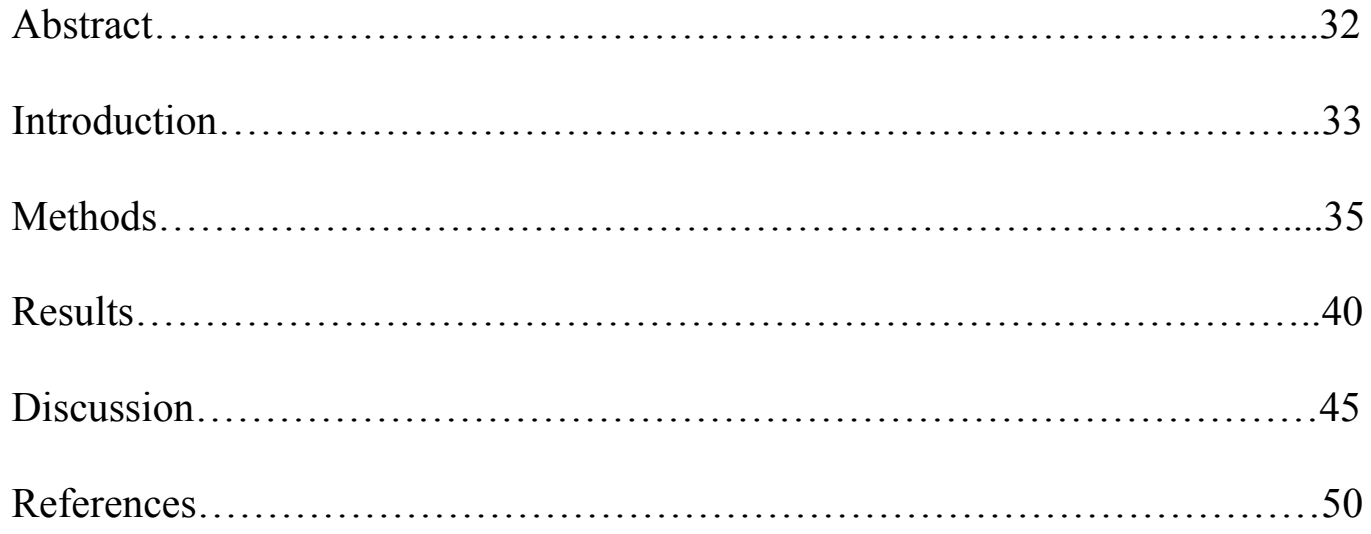

Chapter 2: Impact of Repetition Number on Muscle Performance and Histological Response

Abstract. .70 


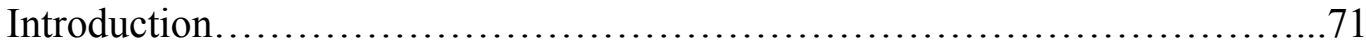

Methods............................................................... 74

Results............................................................. 79

Discussion........................................................... 80

References.......................................................... 84

Chapter 3: Adaptive Stretch-Shortening Contractions: Diminished Regenerative Capacity with Aging

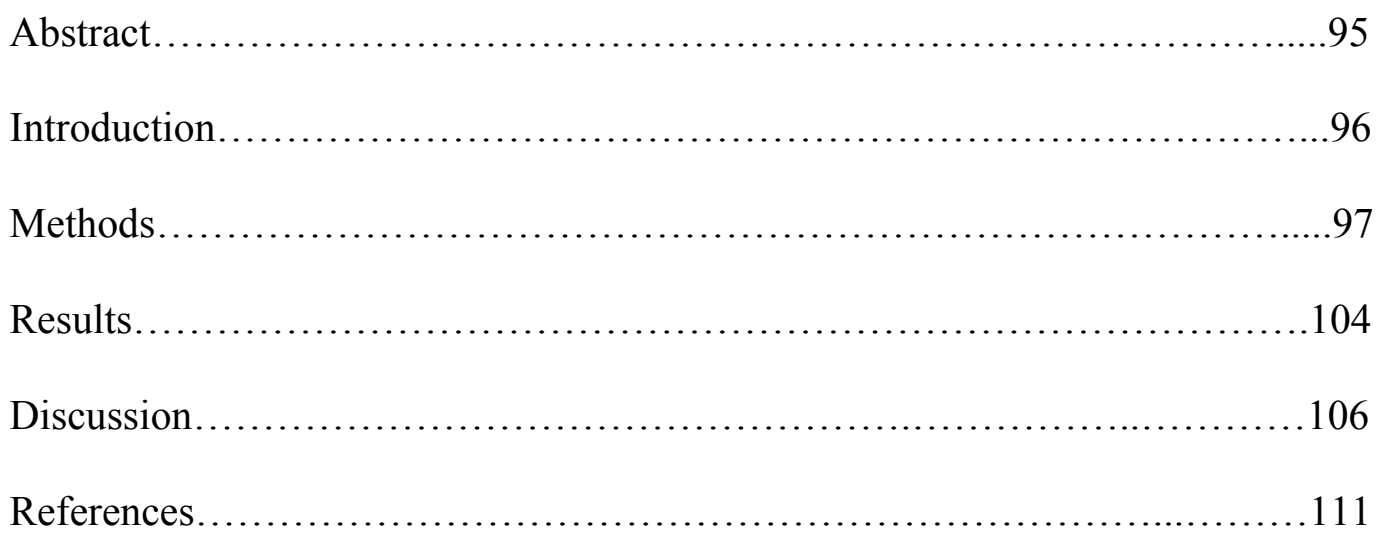

Chapter 4: Chronic Exposure to Stretch-Shortening Contractions Results in Skeletal Muscle Adaptation in Young Rats and Maladaptation in Old Rats

Abstract............................................................ 131

Introduction............................................................ 132

Methods........................................................... 134

Results........................................................141

Discussion......................................................... 145 
References.

Chapter 5: Effects of Glutathione Depletion and Age on Performance and Skeletal Muscle Morphology Following Chronic Stretch-Shortening Contraction Exposure

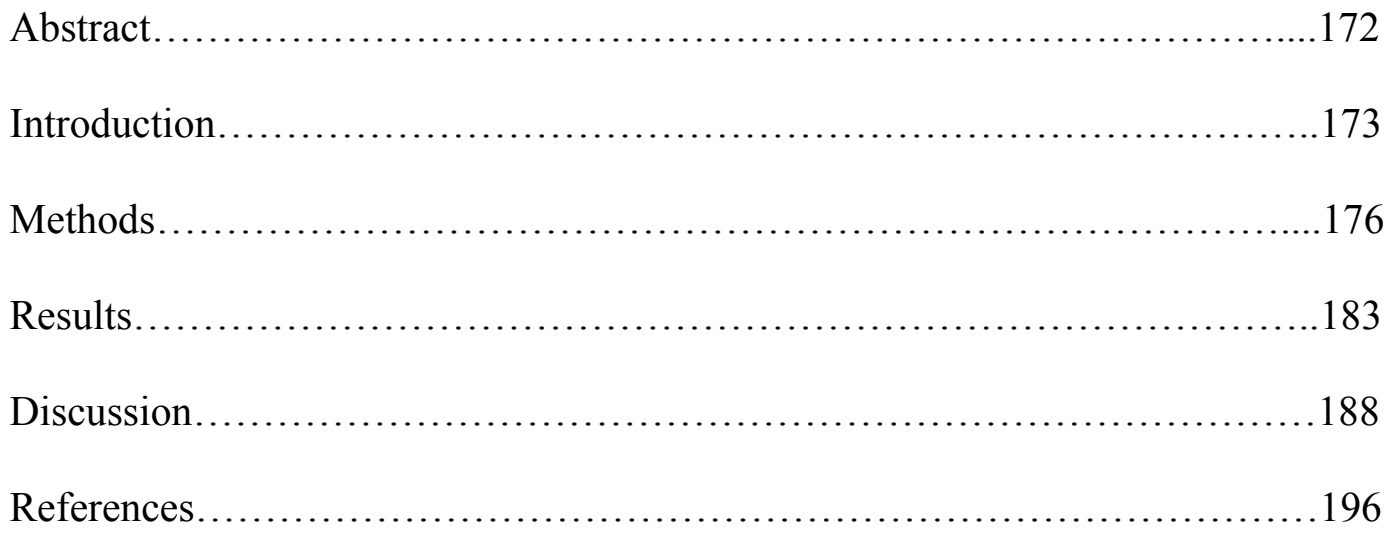

Chapter 6: Myosin Heavy Chain Immunohistochemistry and Fiber Adaptation Following Chronic Stretch-Shortening Contraction Exposure: Influence of Glutathione Modulation and Age

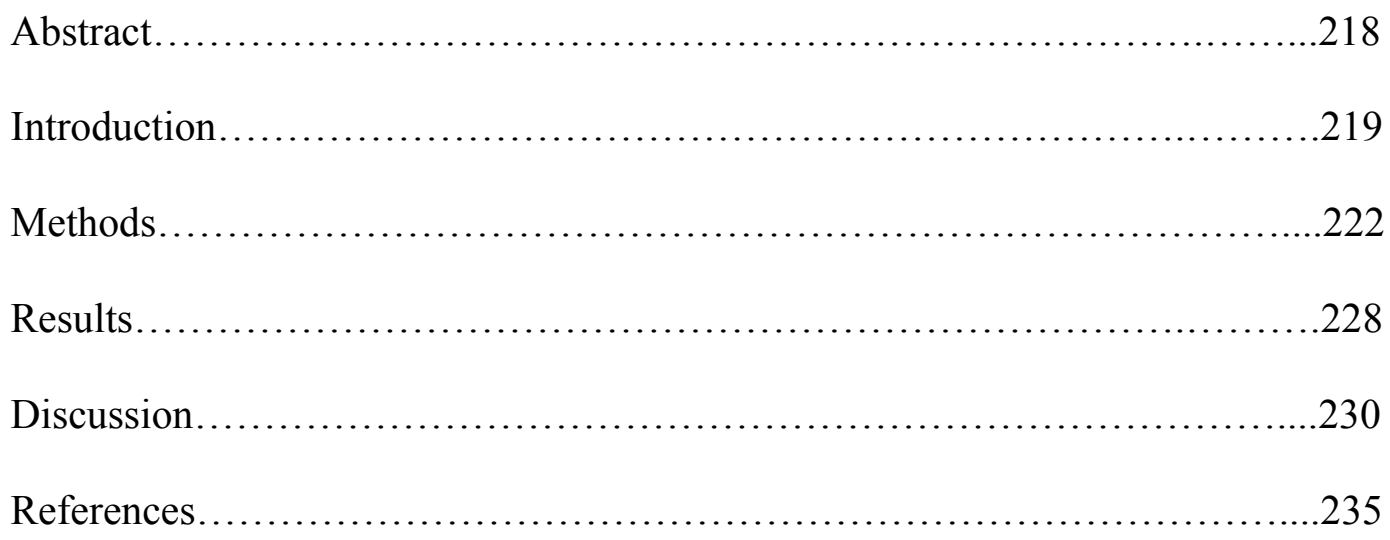

General Discussion...................................................251

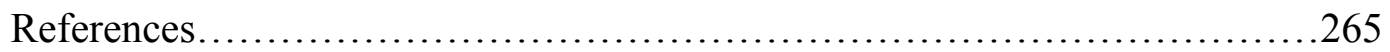




\section{Lists of Figures}

\section{Background and Significance}

Figure 1: In Vivo Rodent Dynamometer....................................29

Figure 2: The Glutathione Pathway: GSH Inhibition via BSO ...................30

\section{Chapter 1:}

Table 1: Absolute and Relative TA Muscle Weights.

Figure 1: Representative TA Photomicrographs Defining Normal and Degenerative Myofibers..................................................58

Figure 2: Isometric Force Analyses........................................59

Figure 3: Normalized Tibialis Anterior Muscle Wet-weights...................60

Figure 4: Representative TA Hematoxylin and Eosin Micrographs..............61

Figure 5: Stereology Analyses: Volume Density .......................................62

Figure 6: Stereology Analyses: Average Thickness.........................63

Figure 7a: Response Kinetics..........................................64

Figure 7b: Response Kinetics.......................................65

Figure 8a: Response Kinetics......................................66

Figure 8b: Response Kinetics.......................................67

Figure 8c: Response Kinetics.....................................68

\section{Chapter 2:}

Figure 1: Representative Drawing of TA muscle and Cross-sectional Sampling...89 
Figure 2: Isometric Force Analyses........................................99

Figure 3: Stereology Analyses: Volume Density.............................91

Figure 4: Stereology Analyses: Volume Density...............................92

Figure 5: Representative TA Hematoxylin and Eosin Micrographs.................93

\section{Chapter 3:}

Table 1: Body Weights and Muscle Wet-weights............................118

Figure 1: Isometric Force Analyses.........................................119

Figure 2a and 2b: Single SSC Force Analyses..............................120

Figure 3a and 3b: Single SSC Work Analyses.................................121

Figure 4: Normalized Muscle Wet-weights................................122

Figure 5a and 5b: Muscle Quality ........................................123

Figure 6: Representative TA Hematoxylin and Eosin Micrographs.................124

Figure 7a and 7b: Stereology Analyses: Volume Density.......................125

Figure 7c and 7d: Stereology Analyses: Volume Density......................126

Figure 8a: Representative $\mathrm{MHC}_{\text {dev }}{ }^{+}$Micrographs..............................127

Figure 8b: Stereology Analyses: $\mathrm{MHC}_{\mathrm{dev}}{ }^{+}$Immunohistochemistry................128

\section{Chapter 4:}

Figure 1a and 1b: Representative Force and Position Tracings.....................161

Figure 2: Temporal Isometric Force Analyses................................162

Figure 3a and 3b: Temporal Single SSC Force Analyses........................163

Figure 4a and 4b: Temporal Single SSC Work Analyses.........................164 
Figure 5: Normalized TA Muscle Wet-weights.............................. 165

Figure 6a and 6b: Representative TA Hematoxylin and Eosin Micrographs.........166

Figure 7: TA Muscle Fiber Histograms....................................167

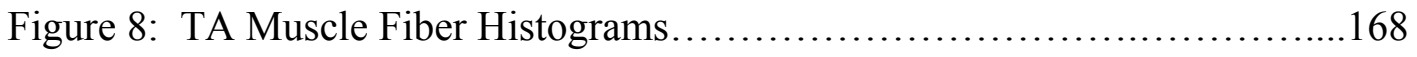

Figure 9: Muscle Quality.............................................169

Figure 10: Stereology Analyses: Volume Density...........................170

\section{Chapter 5:}

Figure 1a and 1b: Single SSC Peak Force Analyses........................203

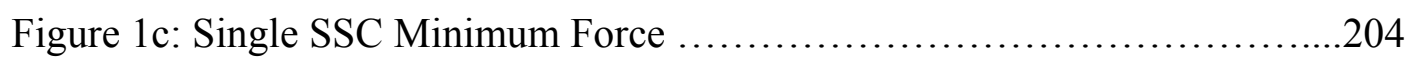

Figure 2a and 2b: Single SSC Work Analyses..............................205

Figure 2c: Single SSC Work Analyses...................................206

Figure 3: Normalized Tibialis Anterior Muscle Wet-weights..................207

Figure 4: Muscle Quality.............................................208

Figure 5a and 5b: Stereology Analyses: Volume Density.....................209

Figure 5c and 5d: Stereology Analyses: Volume Density.....................210

Figure 6a-6d: Representative TA Hematoxylin and Eosin Micrographs........211

Figure 7a-7d: Representative TA Hematoxylin and Eosin Micrographs.........212

Figure 8a: TA Muscle Fiber Histograms..................................213

Figure 8b: TA Muscle Fiber Histograms.................................2 214

Figure 8c: TA Muscle Fiber Histograms. ..............................2 215

Figure 8d: TA Muscle Fiber Histograms .................................216 


\section{Chapter 6:}

Figure 1a and 1b: TA Muscle Cumulative Frequency Histograms..............241

Figure 1c: TA Muscle Cumulative Frequency Histograms .....................242

Figure 2a and 2b: TA Muscle Cumulative Frequency Histograms ..............243

Figure 2c: TA Muscle Cumulative Frequency Histograms ......................244

Figure 3a-3d: Representative $\mathrm{MHC}_{\text {slow }}{ }^{+}$Micrographs .......................245

Figure 3e and 3f: Representative $\mathrm{MHC}_{\text {slow }}{ }^{+}$Micrographs ......................246

Figure 4: Stereology Analyses: $\mathrm{MHC}_{\text {slow }}{ }^{+}$Immunohistochemistry ..............247

Figure 5a-5d: Representative $\mathrm{MHC}_{\text {fast }}{ }^{+}$Micrographs ......................248

Figure 5e and 5f: Representative $\mathrm{MHC}_{\text {fast }}{ }^{+}$Micrographs .....................249

Figure 6: Stereology Analyses: $\mathrm{MHC}_{\text {fast }}{ }^{+}$Immunohistochemistry ................250 


\section{$\underline{\text { Specific Aims }}$}

The increased incidence of contraction-induced muscle injury that occurs in the workplace is not well explained, but it has been approximated that there are 700,000 lost time incidents per year in the United States due to repetitive-strain injury. Additionally, given that the related health-care costs for musculo-skeletal disorders reaches into the billions each year (Reville et al., 2002), it is remarkable that a clear understanding of the underlying mechanisms that regulate the target muscle/s plasticity with respect to acute and repetitive mechanical loading does not exist. Thus, it is prudent that our awareness of the functional, physiological, and biochemical processes that allow for adaptation or result in mal-adaptation or overt skeletal muscle injury to acute and repetitive mechanical loading is enhanced. Another factor that must be considered in workplace settings is the age of an individual, especially since the current workforce is aging at a significant rate ((NRC) and (IM), 2001, Statistics, 1999). Thus, as the workforce ages and susceptibility to contraction-induced injury increases, there becomes a greater need for a more concise understanding detailing both injury and adaptive thresholds for acute and repetitive mechanical loading environments. Furthermore this essential concept must be acknowledged and accounted for with respect to the aging population as well as for developing and modifying preventive strategies such as those that modulate the oxidative and inflammatory environment of the host organism in the absence of overt soft-tissue injury. Since aging is characterized by a progressive deterioration in various physiological functions and metabolic processes, the involvement may be accredited to changes in genetic machinery as well as "wear and tear" mechanisms such as, increased oxidative stress, increased inflammation, and/or muscle fiber phenotype plasticity. Collectively, a significant void continues to be apparent in our understanding of how the involvement of these multiple factors is integrated into affecting muscular adaptation. 


\section{Central Hypothesis}

Characterizing and determining time- and dose-dependent contraction-induced injury responses in skeletal muscle following acute mechanical loading exposure. Additionally, in the absence of skeletal muscle degeneration, this project will identify and quantify acute adaptive events in muscle regeneration and determine the role age has on this response. Finally, the adaptability of skeletal muscle in young and old rodents will be determined following chronic, repetitive mechanical loading exposures, and it will be ascertained if modulating the glutathione system by decreasing glutathione levels in skeletal muscle of young and old rodents will diminish the adaptive profile following chronic loading exposures. The overall objective of this study will elucidate: 1) the time- and dose-response relationship of muscle degeneration, the inflammatory response, and repair and regeneration responses associated with acute mechanical loading, 2) the impact aging has on the acute temporal response to adaptive SSC loading, 3) if aging influences the adaptive response of skeletal muscle following chronic SSC loading, 4) if depletion of the glutathione system and the oxidative stress/antioxidant profile impacts skeletal muscle adaptation, and 5) if the role age has on early muscle regeneration following acute SSC loading. This project will investigate fundamental functional parameters (muscle isometric and dynamic forces), muscle morphology, and cellular biochemistry in aging muscle tissue following acute and chronic, repetitive stretch-shortening contraction (SSC) exposures. This research will also evaluate if mal-adaptation is inherent to aging or influenced by the mismanagement of oxidative stress, and possible factors that account for differences previously observed with aging that are involved in early skeletal muscle regeneration. In order to achieve these objectives, the specific aim and hypotheses below are designed to quantitatively assess these voids. Each specific aim is 
stated with a brief description of how each specific aim will be accomplished.

$\underline{\text { Specific Aim 1: }}$ To systematically quantify skeletal muscle degeneration and inflammation and changes occurring to the interstitial space in a time-dependent manner, and determine which parameters could be related to the changes in muscle performance.

- Method: Young rodents will be exposed to an acute bout of $150 \mathrm{SSCs}$ in vivo, and muscle performance and muscle morphology will be quantified temporally.

- Hypothesis 1.1: Indices of myofiber degeneration and inflammation, as well as modifications observed in the interstitial space, will be increased temporally as a result of the SSC-induced muscle injury in young rodents.

Hypothesis 1.2: Quantitative measures of muscle morphology will correlate with changes in muscle functional performance temporally in young rodents.

Specific Aim 2: To determine the effects that increasing repetition number has on skeletal muscle functional performance 48 hours following an acute SSC exposure, and to quantify the dose-dependent effects increasing repetition number has on skeletal muscle morphology 48 hours following an acute SSC exposure.

- Method: Young rodents will be exposed to an acute bout of increasing numbers of SSCs in vivo, and muscle performance and muscle morphology will be quantified at 48 hours.

- Hypothesis 2.1: Young rodents exposed to increasing numbers of SSC 
repetitions will have increased functional performance decrements 48 hours following acute SSC exposure.

- Hypothesis 2.2: Young rodents exposed to increasing numbers of SSC repetitions will have increased stereological indices of myofiber injury, inflammation, and increased disruption to the interstitial space 48 hours following acute SSC exposure.

Specific Aim 3: To quantify the acute performance and myofiber changes that are responsible for initiating the adaptive response in young and old rodents following an adaptive SSC exposure temporally.

- Method: Young and old rodents will be exposed to an acute bout of adaptive SSCs in vivo, and performance, muscle morphology, and developmental myosin heavy chain expression will be quantified temporally.

- Hypothesis 3.1: Young and old rodents exposed to an acute bout of adaptive SSCs will demonstrate functional loss following acute, adaptive SSC exposure.

- Hypothesis 3.2: Young and old rodents will exhibit indices for myofiber degeneration and inflammation, and both will exhibit increased muscle regeneration 120 hours following SSC exposure.

Specific Aim 4: To determine if aging affects skeletal muscle safety threshold or ability to adapt to chronic, repetitive SSC exposure, and to quantify the isometric and dynamic performance 
during the exposure period as well as myofiber plasticity following the completion of the chronic exposure period.

- Method: Young and old rodents will be exposed to a 4.5 week chronic bout of adaptive SSCs in vivo, and performance and muscle morphology will be quantified following exposure.

- Hypothesis 4.1: Young rodents will adapt to chronic, repetitive SSC exposures, whereas old rodents will not adapt to the same chronic loading protocol as evidenced by a lower muscle safety threshold or tolerance

Specific Aim 5: To determine the influence dietary supplementation of $10 \mathrm{mM}$ L-Buthionine Sulfoximine (BSO), a glutathione antagonist (Griffith and Meister, 1979), has on the adaptability of young and old rat tibialis anterior muscle during chronic, repetitive SSC exposure. Since BSO is a glutathione antagonist and an irreversible inhibitor of $\gamma$-glutamylcysteine synthase (GCS) the rate-limiting enzyme for glutathione synthesis, the overall antioxidant profile will be diminished. The significance of supplementing the diet with a glutathione agonist will be assessed both functionally and morphologically.

- Method: Young and old rodents will be supplemented with BSO in their drinking water and exposed to 4.5 weeks of adaptive SSCs in vivo, and performance and muscle morphology will be quantified following exposure.

- Hypothesis 5.1: Glutathione depletion will decrease the performance of rat dorsi-flexor muscles in young and old rodents exposed to chronic SSC exposures.

- Hypothesis 5.2: Glutathione depletion will increase morphological indices 
of inflammation and degeneration in young rodents and exacerbate this response in old rodents exposed to chronic SSC exposures.

- Hypothesis 5.3: Glutathione depletion will decrease skeletal muscle mass and myofiber cross-sectional area in young and old rodents exposed to chronic SSC exposures.

Specific Aim 6: To determine the influence glutathione depletion has on the adaptability of skeletal muscle myosin heavy chains and on muscle morphology with aging following chronic SSC exposure.

- Method: Young and old rodents will be supplemented with BSO in their drinking water and exposed to 4.5 weeks of adaptive SSCs in vivo, and myosin heavy chain immunohistochemistry will be quantified following exposure.

- Hypothesis 5.1: Skeletal muscles undergoing glutathione depletion and chronic SSC exposure will not express developmental myosin as a result of decreased muscle regeneration.

- Hypothesis 5.2: The population of small myofibers that exist in old rodents following SSC exposure will not express developmental myosin, because these fibers contribute to age-related mal-adaptation. 


\section{Background and Significance}

\section{$\underline{\text { Aging Perspective }}$}

The National Occupational Research agenda has identified musculo-skeletal injury mechanisms as one of the primary focus areas in the United States. Coincidently, the age distribution of the workforce in the United States is predicted to be shifting to an older population, and the 55-64 year old demographic is now the fastest growing sector of the labor force in the United States (Statistics, 1999). In fact, as of last year, 20 percent of the workforce has now become comprised of workers over the age of 55 years of age ((NRC) and (IM), 2001).

It is commonly accepted that aging results in a loss of muscle fibers and atrophy of the remaining muscle fibers (this contributes to the overall loss of muscle mass), decreased endurance capacity, decreased fatigue resistance as well as a decrease in strength and force production (Leveille, 2004, McArdle et al., 2002). Specifically, the age-related loss of skeletal muscle mass has been termed sarcopenia (Evans, 1995), as muscle mass (reported by decreased cross-sectional area) has been shown to decrease by approximately $30-40 \%$ in old versus young subjects and has been reviewed extensively (Porter et al., 1995, Vandervoort, 2002, Doherty, 2003) . Sarcopenia is an age driven phenomenon, because all elderly individuals have decreased muscle mass and strength compared to healthy younger individuals (Roubenoff, 2001). Accompanying this loss of muscle mass is the loss of muscle strength (decrease in force production), which typically results in a $2040 \%$ reduction in muscle strength (Doherty, 2003). Contributing to the onset and progression of sarcopenia are a number of mechanisms including: denervation (Larsson et al., 1979, Jackson et al., 1984), loss of motor units (Doherty et al., 1993), an increased production of radical and non-radical species (more specifically known as reactive oxygen species -ROS and reactive nitrogen species - RNS) in resting and working muscle (Ji 
and Peterson, 2004, Weindruch, 1995), contraction-induced muscle injury (Faulkner et al., 1995), death of myofibers either by degeneration/necrosis or apoptosis (Brooks and Faulkner, 1996) accompanied by a decline in satellite cell turnover and myofiber regeneration (Grounds, 1998), and, the more recent hypothesis, mitochondrial dysfunction (Alway et al., 2002, Dirks and Leeuwenburgh, 2002, Aiken et al., 2002) with preferential apoptosis of muscle satellite cells in aged tissue (Siu and Alway, 2005, Krajnak et al., 2006). Thus, due to time itself being associated with the aging process, it is not clear if the death of skeletal muscle fibers is inherently regulated by the multi-factorial aging process, or more specifically by singular, acute events that are repeated regularly. Nonetheless, these age-related changes in skeletal muscle may make the tissue less capable of responding to repetitive stressors (Brooks and Faulkner, 1994, Cutlip et al., 2006). In addition, the functional relevance of a sarcopenic state mentioned above may have multiple underlying mechanisms. Since it is well known that skeletal muscle force production (which translates into the ability to produce muscular strength and power) is directly proportional to the skeletal muscle cross-sectional area, events that play a role in the reduction of skeletal muscle mass will be intimately tied to functional loss.

\section{Contraction-Induced Skeletal Muscle Injury}

Single stretches as well as repetitive muscular contractions, or stretch-shortening contractions (SSCs), may lead to overt skeletal muscle injury (inflammation, myofiber degeneration, and dysfunction) skeletal muscle adaptation (regeneration and growth with functional gains), and/or mal-adaptation (a sub-degenerative or sub-necrotic state that is usually associated with low levels of persistent inflammation as well as loss of function); however, none of these processes are likely to be mutually exclusive. In most cases, muscles compensate for increased demands in a 
systematic fashion, yet situations do arise in which the muscle does not adequately meet those demands; this leads to overt skeletal muscle injury. Exposure to concentric (shortening) or isometric muscle actions does not normally produce muscle injury (Lieber et al., 1996, Baker et al., 2006b, Warren et al., 1993a, Faulkner et al., 1995). However, exposure to eccentric contractions has been shown to result in an isometric force deficit (Warren et al., 1999a), structural disruption at the cellular level, and cellular infiltrates as a result of an inflammatory response (Lieber et al., 1996, Faulkner et al., 1989, Friden et al., 1983a, McCully and Faulkner, 1985). Also it has been shown that exposure to injurious eccentric muscle actions results in disruption of the cellular membrane, loss of intermediate filaments and structural proteins, and the influx of extracellular proteins into the cell (Komulainen et al., 1998, Komulainen et al., 2000, Friden and Lieber, 1998, Lieber et al., 1994). Sarcomeric lesions, disorganized actin, and Z-disc streaming also result after injury (Stupka et al., 2001, Vijayan et al., 2001, Devor and Faulkner, 1999, Lieber et al., 1991). During the injury process, damaged cells lose apposition to neighboring cells and there is evidence of cellular infiltrates such as neutrophils and macrophages entering damaged myofibers (Koh et al., 2003, Devor and Faulkner, 1999). During this time satellite cells (quiescent muscle precursor cells) are activated, proliferate, differentiate, and finally fuse with the existing myofiber (Charge and Rudnicki, 2004, Hawke and Garry, 2001). Further, developmental myosin heavy chain is expressed in injured fibers during this time period, and this has been suggested to comprise the recapitulation of the developmental program (McCormick and Schultz, 1994). At this time, the muscle demonstrates a mixture of both degenerative and regenerative processes. Finally, central nuclei appear and are present at extended time points following the initial exposure indicating resolution from previous injury (Bigard et al., 1997a, Hesselink et al., 1996). Even though numerous models have been used to 
study the functional and biological mechanisms of contraction-induced muscle injury, there is a paucity of studies quantifying both skeletal muscle damage and inflammation after contractioninduced muscle injury (Koh et al., 2003, Geronilla et al., 2003). Recently, the ability to rapidly quantify both skeletal muscle degeneration and inflammation in the same tissue following an injurious exposure has been shown to reveal further insight into the injury and repair process of skeletal muscle (Baker et al., 2006a, Baker et al., 2006b, Baker et al., In press-a, Cutlip et al., 2006) (Baker et al., In review 2007).

\section{Contraction-Induced Skeletal Muscle Injury: Influence of Age}

It is clear that skeletal muscle's susceptibility to contraction-induced injury is increased with age in both humans (Manfredi et al., 1991) and animals (Brooks and Faulkner, 1996). This is indicated by an increased force deficit (Koh et al., 2003, Brooks and Faulkner, 1996, Zerba et al., 1990) and slower recovery of performance measures in old versus young animals following exposure (McBride et al., 1995, Sacco and Jones, 1992, Manfredi et al., 1991, Brooks and Faulkner, 1990). Thus, it is clear that aging impairs the ability of skeletal muscle to adapt to chronic mechanical loading. While most studies conducted to-date have investigated the effect of age on the performance response and morphological changes after a single injurious exposure of eccentric contractions, there are very few studies that have investigated age effects on muscle degeneration and adaptation due to chronic high-intensity mechanical loading using SSCs (Cutlip et al., 2006).

What is quite evident is the disparity that exists in the previous literature regarding the effects of age on skeletal muscle's adaptive/mal-adaptive response following chronic mechanical loading. Moreover, chronic exposure paradigms have focused on aerobic means of exposure to 
investigate the adaptive response. There is evidence that 10 weeks of treadmill training attenuated eccentric muscle damage in vitro in both young and older rats (Gosselin, 2000). Further, Leeuwenburgh and Ji found that exhaustive swim exercise in the presence of glutathione depletion exacerbated glutathione status, and glutathione homeostasis was critical for oxidant/antioxidant regulation following exposure (Leeuwenburgh and Ji, 1995). Even though under-utilized, chronic mechanical exposures have been employed in an attempt to understand how the adaptive process is regulated. Previously, 6 weeks of eccentric contractions provided a protective effect by preventing a substantial force deficit and morphological evidence of damage in muscles from both young and old animals (Brooks et al., 2001) exposed to a protocol that is injurious in an acute exposure. However, while both adult and old mice adapted successfully to the conditioning protocol (1 bout of eccentric contractions per week), the older mice adapted more slowly than the younger adult mice (Brooks et al., 2001). While these studies showed that muscles from older animals could be conditioned to be protected from eccentric contractioninduced injury, the conditioning stimulus was inadequate to promote hypertrophy of the target muscles or increase performance.

\section{High-Intensity Mechanical Loading Paradigm (Adaptive Exposure)}

As with the studies eluded to above, the considerable body of existing evidence involving the comprehensive analysis of skeletal muscle comes from animal studies, due to the challenges that arise with accessibility and feasibility of testing in human populations, and this has made animal models appealing for laboratory testing (Alway et al., 2005). Even though studying aging in humans is not easy, the scientific literature indicates that animal models are appropriate for the investigation of skeletal muscle adaptation and injury. Moreover, natural movement is 
comprised of SSCs (reciprocal concentric and eccentric muscle actions), and are an effective means to introduce high-intensity mechanical loading (i.e. resistance exercise) in skeletal muscle, and in vivo rodent dynamometry (depicted in Figure 1) is an effective means to evaluate skeletal muscle's response to mechanical loading (Cutlip et al., 1997a). SSCs have been studied in the context of human locomotion and athletic performance (Avela and Komi, 1998) and have been shown to produce muscle injury due to the eccentric component of the cycle (Horita et al., 1999). Since natural muscle function is comprised of SSCs, this approach provides an improved physiologically relevant exposure model over the traditional eccentric-only injury model (Komi, 2000). The use of anesthetized rats allows for controlled stimulation of the muscle group of interest and rigorous functional, morphological, and biochemical analyses of the muscle tissue following exposure (Lieber et al., 1996, Stauber and Smith, 1998, Warren et al., 1991). The micro-architecture of rodent and human skeletal muscle is quite similar, and, in previous studies, the human and rodent response to acute injury resulted in similar performance decrements, morphological changes, and biochemical expression (Clarkson and Tremblay, 1988, Jones et al., 1986). It has been reported that mechanical loading results in skeletal muscle injury and is associated with increased oxidative stress levels (Brickson et al., 2001, Clarkson and Hubal, 2002). Since aging by itself increases the oxidative stress level in skeletal muscle (Gianni et al., 2004) the detrimental effects may be exacerbated with the addition of chronic mechanical loading exposures; this may preclude the target muscle from properly undergoing degeneration, apoptosis, repairing, or adapting (Dirks and Leeuwenburgh, 2004). Historically, the characterization of skeletal muscle plasticity over the past 20-30 years has been focused on muscle repair and regeneration following events producing overt muscle injury. 
Once again, the effects of high-intensity mechanical loading and the subsequent response of muscle in animals and humans have focused on the acute effects of injurious muscular exposures (Brooks and Faulkner, 1990, Brooks and Faulkner, 1996, Bejma and Ji, 1999), or on endurance-type exposures (Leeuwenburgh et al., 1994, Gore et al., 1998, Fitts et al., 1984). These studies demonstrate that muscles from older animals are more likely to be injured as a result of the exposure, and less likely to recover after exposure to an acute bout of force generating eccentric contractions (Brooks and Faulkner, 1990, Brooks and Faulkner, 1996). However, it is unclear if during all mechanical loading movements an individual is undergoing damaging muscular contractions. In this scenario (no significant muscle degeneration) there is likely to be a greater influence of oxidative stress-induced alterations, versus what classically has been described with overt skeletal muscle injury (McArdle et al., 2001). Likewise, numerous occupations (e.g. construction work, manufacturing work, automobile assembly), where workers endure repetitive muscular movements that require the generation of high levels of force during the execution of SSCs occur daily. In effect, the worker is continually in an adaptive phase (be that either positive or negative) where the stressor may be stimulating various pathways leading to increased levels of oxidative stress, inflammation, degeneration/apoptosis, and repair and regeneration mechanisms in the activated target muscle/s. Collectively, there are few studies that have examined the long-term impact on the adaptive response of aging skeletal muscle by manipulation of the antioxidant system following chronic SSC exposures, and this void must be addressed.

\section{Oxidative Stress and Mechanical Loading}


Mechanical loading exposures (i.e. resistance-type exercise) have been shown to increase the production of ROS/RNS, and this may exceed the naturally occurring endogenous levels of antioxidant defenses within an organism (Davies et al., 1982). Numerous studies have indicated that oxidative stress may at least in part play a role in aging-induced skeletal muscle maladaptation (Ji et al., 1998, Bejma and Ji, 1999), and recent data from our lab is in general agreement with this concept, which hypothesizes that the inability to cope with oxidative stress and the ensuing inflammatory cascade may be a key limiting factor in adaptability, especially in aged populations (McArdle and Jackson, 2000, Cutlip et al., 2006). Moreover, the lack of adaptation observed with aged animals is likely to be attributed to the inability to effectively manage oxidative stress, since reactive oxygen species and/or reactive nitrogen species (ROS/RNS) are constantly generated in active muscle cells and oxidative stress increases if these species are not properly countered by antioxidant defenses. Since skeletal muscle represents the largest pool of total glutathione in the body, it is plausible that glutathione is the most important non-protein thiol source that maintains most cells in a reduced environment (Meister, 1991). Because of glutathione's role as a major antioxidant in the cell, it serves various functions such as directly acting as a reducing agent, serving as a substrate for glutathione peroxidase (and others), and recycling radicals generated from other antioxidant molecules, the adaptive responses ensuing from modulation of the glutathione system may be ascertained. Glutathione depletion can be accomplished using L-Buthionine Sulfoximine (BSO), which is an irreversible inhibitor of $\gamma$-glutamylcysteine synthase (GCS, arrow - Figure 1) (Meister, 1991). In a recent study from our lab, young and old rodents were supplemented with either BSO (10mM in water) or placebo (normal water) and measures of oxidative stress were quantified (Hollander et al., in preparation). BSO was effective in decreasing total glutathione levels in the target musculature, 
irrespective of age. Additionally, BSO treatment increased measures of hydrogen peroxide in both young and old rodents (indicating increased oxidative stress), yet this increase in hydrogen peroxide was prevented with chronic, repetitive SSC exposure in young rodents only. Also chronic SSC exposure increased indices of lipid peroxidation and cell death in aged rodents only, but had no effect on hydrogen peroxide, irrespective of age. Given that oxidative stress is primarily buffered in muscles of young organisms, limited functional impairment and minimal detrimental effects to the cell may be encountered. However when the body's capacity to buffer oxidative stress is compromised, such is the case with aging, oxidative stress may influence maladaptive cellular processes. Part of this mal-adaptation observed with aging that occurs with chronic mechanical loading may be due to an imbalance in oxidative stress in the target skeletal muscle/s, as several studies have indicated that oxidative stress participates in this adaptive response by imparting changes in various gene pathways (Wretman et al., 2001, Jackson et al., 2002). Oxidative stress is greater in metabolically active tissues (i.e. skeletal muscle) from aged animals and humans, so it may contribute to the higher level of mal-adaptation in skeletal muscle of aged animals. Events that increase oxidative stress, such as mechanical loading and increased physical activity may also decrease the functional threshold (tissue tolerance) by negatively influencing the host's internal cellular environment; however these questions have not been systematically investigated. Yet, it is for this reason antioxidant supplementation has been proposed as an approach to counter the negative effects of increased oxidative stress and subsequent damage. Numerous antioxidants (vitamins, proteins, enzymes and pathways) are involved in attenuating oxidative-induced damage, and a few of the most commonly studied have been vitamin C, vitamin E, n-acetylcysteine, superoxide dismutase (SOD), catalase, glutathione peroxidase, glutathione reductase. This may be exemplified as oxidative stress can influence 
reduction-oxidation pathways as well as other inducible antioxidant systems (such as the glutathione system). In preliminary studies from our lab, both a vitamin $\mathrm{C} \& \mathrm{E}$ combination dietary regime as well as dietary supplementation with curcumin enhanced functional and morphological adaptation as well as decreased estimates of cellular infiltrates in old rodents compared with old rodents that received no dietary countermeasures (unpublished data). Together these data indicate that in old rodents dietary supplementation with antioxidants may prove beneficial. BSO has been documented both physiologically and biochemically to chronically deplete levels of glutathione, and BSO would be expected to result in a more vulnerable state to mechanical loading-induced elevations in oxidative stress (Martensson, 1989, Leeuwenburgh and Ji, 1995) (Hollander et al., In preparation). The adaptability of many antioxidant pathways are influenced by regular physical activity and have been shown to impact a range of adaptive responses following chronic exercise in skeletal muscle adaptation (Ji, 1995, Ji et al., 1998, Radak et al., 2005, Powers et al., 1999).

\section{Skeletal Muscle Adaptation (Hypertrophy/Hyperplasia)}

Oxidative stress results from increased free radical production from numerable sources following physical exposure and has been demonstrated to produce negative changes in functional (McArdle et al., 2001) and cellular responses (McBride, 1998, McArdle et al., 1999) which is exacerbated in aged skeletal muscle (Zerba et al., 1990). More specifically, ROS has been suggested as a contributing mechanism to the injurious response observed in skeletal muscle following resistance training/mechanical loading (Uchiyama et al., 2006). However, others have suggested that oxidative stress may also be a critical stimulus for skeletal muscle adaptation (Urso and Clarkson, 2003, McArdle et al., 2002), since the oxidative stress profile influences 
various adaptive pathways in muscle repair and regeneration (McArdle et al., 2004, McArdle et al., 2001, McArdle et al., 2006). During and following the repair and regeneration phase in postnatal skeletal muscle, increased skeletal muscle mass is accomplished primarily via increases in contractile tissue. New contractile proteins are added in one of two ways: 1) in series by the addition of new sarcomeres and 2) in parallel by the addition of myofibrils. The contribution that hyperplasia imparts on increasing skeletal muscle mass has been a matter of contention, since conflicting data exists illustrating hyperplasia’s involvement (Gollnick et al., 1981, Gonyea, 1980, Alway et al., 1989) Nevertheless, it is the normally quiescent satellite cell (muscle stem cell) (Mauro, 1961), located between the plasma membrane and basement membrane of myofibers, that has intrinsic mitotic activity and contributes myonuclei to regenerating and hypertrophying skeletal muscles (Moss and Leblond, 1970, Darr and Schultz, 1987). The addition of satellite cells and sarcomeres to existing myofiber of a target muscle results in increased myofiber size, and it has been shown that the "type" of sarcomere that is added following periods of increased muscular activity is of the slow-type myosin, regardless of the existing myosin composition of the target muscle (Williams et al., 1986). Since there are countless factors influencing the adaptive process following increased use, it is sensible to direct one's focus on a specific feature of this dynamic process. Myosin heavy chain (MHC) expression is modulated in young and aged populations in response to altered neural and functional demands (Roy et al., 1997, Alway et al., 1995), and may be influential in regulating the onset of the adaptive response (Smith et al., 1999). In addition to the regulation of slow-type myosin stated above, it has been reported that following muscle injury, electrical stimulation, and/or mechanical stretch to tissue, the developmental MHC isoform is re-expressed and is an important factor in the regenerative and adaptive state (Lieber et al., 1994, Alway et al., 1995, 
Smith et al., 1999). In particular, it has been reported that following a single bout of exerciseinduced muscle injury, there is a transient increase in the expression of developmental MHC in small, regenerating myofibers (Smith et al., 1999). However, others have shown that there is an increase in the expression of developmental MHC following periods of chronic mechanical loading in which hypertrophy is present, and this expression occurs in mature myofibers that are not undergoing degeneration/ regeneration (Phelan and Gonyea, 1997). Consequently, chronic SSC exposure may lead to changes in MHC expression and distribution with age that ultimately impacts muscle hypertrophy.

\section{Summary}

The characterization and quantification of muscle performance and histopathology in contraction-induced skeletal muscle injury by time- and dose-dependent responses as well as the capacity and mechanisms influencing muscle and interstitial space repair, remodeling, and regeneration must be precisely determined for acute mechanical loading paradigms and the influence age has on these responses. Subsequently, these same factors should be explored with the use of chronic, repetitive mechanical loading models to determine both adaptive and injury thresholds of skeletal muscle, as well as to establish the safety threshold or tolerance of skeletal muscle with repeated loading exposures. However, in the absence of overt skeletal muscle injury mismanagement of oxidative stress may contribute to the adaptive/mal-adaptive response exhibited by skeletal muscle. One factor that may be manipulated to observe the functional and biological changes is the oxidant/antioxidant system, specifically glutathione. To date there are no studies that have determined the influence diminished glutathione content in skeletal muscle from young and old rodents has on the adaptive response following chronic SSC exposures that 
are sufficient to promote physiological adaptation and performance increases in skeletal muscle of young, unsupplemented rodents. Finally, identifying the acute events that are responsible for initiating adaptive processes (repair and regeneration), and how these events differ with age in skeletal muscle is important following mechanical loading of skeletal muscle 


\section{$\underline{\text { References }}$}

(NRC), N. R. C. \& (IM), I. F. M. (2001) Musculoskeletal Disorders and the Workplace, Washington, D.C., National Academy Press.

AIKEN, J., BUA, E., CAO, Z., LOPEZ, M., WANAGAT, J., MCKENZIE, D. \& MCKIERNAN, S. (2002) Mitochondrial DNA deletion mutations and sarcopenia. . Ann NY Acad Sci, 959, 412-423.

ALWAY, S., SIU, P., MURLASITS, Z. \& BUTLER, D. (2005) Muscle hypertrophy models: applications for research on aging. Can J Appl Physiol, 5, 591-624.

ALWAY, S. E., CARSON, J. A. \& ROMAN, W. J. (1995) Adaptation in myosin expression of avian skeletal muscle after weighting and unweighting. J Muscle Res Cell Motil, 16, 11122.

ALWAY, S. E., DEGENS, H., KRISHNAMURTHY, G. \& SMITH, C. A. (2002) Potential role for Id myogenic repressors in apoptosis and attenuation of hypertrophy in muscles of aged rats. Am J Physiol Cell Physiol, 283, C66-76.

ALWAY, S. E., WINCHESTER, P. K., DAVIS, M. E. \& GONYEA, W. J. (1989) Regionalized adaptations and muscle fiber proliferation in stretch-induced enlargement. J Appl Physiol, $66,771-781$.

AVELA, J. \& KOMI, P. V. (1998) Reduced stretch reflex sensitivity and muscle stiffness after long-lasting stretch-shortening cycle exercise in humans. Eur J Appl Physiol Occup Physiol, 78, 403-10.

BAKER, B. A., HOLLANDER, M. S., MERCER, R. R., KASHON, M. L., \& CUTLIP, R. G. (In review 2007) Adaptive stretch-shortening contractions: diminished regenerative capacity with aging.

BAKER, B. A., MERCER, R. R., GERONILLA, K. B., KASHON, M. L., MILLER, G. R. \& CUTLIP, R. G. (2006a) Stereological analysis of muscle morphology following exposure to repetitive stretch-shortening cycles in a rat model. Appl Physiol Nutr Metab, 31, 16779.

BAKER, B. A., MERCER, R. R., GERONILLA, K. B., KASHON, M. L., MILLER, G. R. \& CUTLIP, R. G. (In press) Impact of SSC Repetition Number on Muscle Performance and Histological Response. Med Sci Sports Exerc. 
BAKER, B. A., RAO, K. M., MERCER, R. R., GERONILLA, K. B., KASHON, M. L., MILLER, G. R. \& CUTLIP, R. G. (2006b) Quantitative histology and MGF gene expression in rats following SSC exercise in vivo. Med Sci Sports Exerc, 38, 463-71.

BEJMA, J. \& JI, L. L. (1999) Aging and acute exercise enhance free radical generation in rat skeletal muscle. J Appl Physiol, 87, 465-70.

BIGARD, A. X., MERINO, D., LIENHARD, F., SERRURIER, B. \& GUEZENNEC, C. Y. (1997) Muscle damage induced by running training during recovery from hindlimb suspension: the effect of dantrolene sodium. Eur J Appl Physiol Occup Physiol, 76, 4217.

BRICKSON, S., HOLLANDER, J., CORR, D. T., JI, L. L. \& BEST, T. M. (2001) Oxidant production and immune response after stretch injury in skeletal muscle. Med Sci Sports Exerc, 33, 2010-2015.

BROOKS, S. V. \& FAULKNER, J. A. (1990) Contraction-induced injury: recovery of skeletal muscles in young and old mice. Am J Physiol, 258, C436-42.

BROOKS, S. V. \& FAULKNER, J. A. (1994) Isometric, shortening, and lengthening contractions of muscle fiber segments from adult and old mice. Am J Physiol, 267, C50713.

BROOKS, S. V. \& FAULKNER, J. A. (1996) The magnitude of the initial injury induced by stretches of maximally activated muscle fibres of mice and rats increases in old age. $J$ Physiol, 497 ( Pt 2), 573-80.

BROOKS, S. V., OPITECK, J. A. \& FAULKNER, J. A. (2001) Conditioning of skeletal muscles in adult and old mice for protection from contraction-induced injury. J Gerontol A Biol Sci Med Sci, 56, B163-71.

CHARGE, S. B. P. \& RUDNICKI, M. A. (2004) Cellular and molecular regulation of muscle regeneration. Physiol Rev, 84, 209-238.

CLARKSON, P. M. \& HUBAL, M. J. (2002) Exercise-induced muscle damage in humans. Am J Phys Med Rehabil, 81, S52-69.

CLARKSON, P. M. \& TREMBLAY, I. (1988) Exercise-induced muscle damage, repair, and adaptation in humans. J Appl Physiol, 65, 1-6.

CUTLIP, R. G., BAKER, B. A., GERONILLA, K. B., MERCER, R. R., KASHON, M. L., MILLER, G. R., MURLASITS, Z. S. \& ALWAY, S. E. (2006) Chronic exposure of 
stretch-shortening contractions results in skeletal muscle adaptation in young rats and maladaptation in old rats. Applied Physiology, Nutrition and Metabolism, 31, 573-587.

CUTLIP, R. G., STAUBER, W. T., WILLISON, R. H., MCINTOSH, T. A. \& MEANS, K. H. (1997) Dynamometer for rat plantar flexor muscles in vivo. Medical \& Biological Engineering \& Computing, 35, 540-3.

DARR, K. C. \& SCHULTZ, E. (1987) Exercise-induced satellite cell activation in growing and mature skeletal muscle. J Appl Physiol, 63, 1816-1821.

DAVIES, K. J., QUINTANILHA, A. T., BROOKS, G. A. \& PACKER, L. (1982) Free radicals and tissue damage produced by exercise. Biochem Biophys Res Commun, 107, 1198 1205.

DEVOR, S. T. \& FAULKNER, J. A. (1999) Regeneration of new fibers in muscles of old rats reduces contraction-induced injury. J Appl Physiol, 87, 750-6.

DIRKS, A. \& LEEUWENBURGH, C. (2002) Apoptosis in skeletal muscle with aging. Am J Physiol Regul Integr Comp Physiol, 282, 519-527.

DIRKS, A. J. \& LEEUWENBURGH, C. (2004) Aging and lifelong calorie restriction result in adaptations of skeletal muscle apoptosis repressor, apoptosis-inducing factor, X-linked inhibitor of apoptosis, caspase-3, and caspase-12. Free Radic Biol Med, 36, 27-39.

DOHERTY, T. J. (2003) Aging and sarcopenia. J Appl Physiol, 95, 1717-1727.

DOHERTY, T. J., VANDERVOORT, A. A., TAYLOR, A. W. \& BROWN, W. F. (1993) Effects of motor unit losses on strength in older men and women. J Appl Physiol, 74, 868-874.

EVANS, W. J. (1995) What is sarcopenia? J Gerontol A Biol Sci Med Sci, 50, 5-8.

FAULKNER, J. A., BROOKS, S. V. \& ZERBA, E. (1995) Muscle atrophy and weakness with aging: contraction-induced injury as an underlying mechanism. J Gerontol A Biol Sci Med Sci, 50 Spec No, 124-9.

FAULKNER, J. A., JONES, D. A. \& ROUND, J. M. (1989) Injury to skeletal muscles of mice by forced lengthening during contractions. Q J Exp Physiol, 74, 661-70.

FITTS, R. H., TROUP, J. P., WITZMANN, F. A. \& HOLLOSZY, J. O. (1984) The effect of ageing and exercise on skeletal muscle function. Mech Ageing Dev, 27, 161-72. 
FRIDEN, J. \& LIEBER, R. L. (1998) Segmental muscle fiber lesions after repetitive eccentric contractions. Cell \& Tissue Research, 293, 165-71.

FRIDEN, J., SEGER, J., SJOSTROM, M. \& EKBLOM, B. (1983) Adaptive response in human skeletal muscle subjected to prolonged eccentric training. Int J Sports Med, 4, 177-83.

GERONILLA, K. B., MILLER, G. R., MOWREY, K. F., WU, J. Z., KASHON, M. L., BRUMBAUGH, K., REYNOLDS, J., HUBBS, A. \& CUTLIP, R. G. (2003) Dynamic force responses of skeletal muscle during stretch-shortening cycles. Eur J Appl Physiol, 90, 144-53.

GIANNI, P., JAN, K. J., DOUGLAS, M. J., STUART, P. M. \& TARNOPOLSKY, M. A. (2004) Oxidative stress and the mitochondrial theory of aging in human skeletal muscle. Exp Gerontol, 39, 1391-1400.

GOLLNICK, P. D., TIMSON, B. F., MOORE, R. L. \& RIEDY, M. (1981) Muscular enlargement and number of fibers in skeletal muscle of rats. $J$ Appl Physiol, 50, 936-943.

GONYEA, W. J. (1980) Role of exercise in inducing increases in skeletal muscle fiber number. $J$ Appl Physiol, 48, 421-6.

GORE, M., FIEBIG, R., HOLLANDER, J., LEEUWENBURGH, C., OHNO, H. \& JI, L. L. (1998) Endurance training alters antioxidant enzyme gene expression in rat skeletal muscle. Can J Physiol Pharmacol, 76, 1139-45.

GOSSELIN, L. E. (2000) Attenuation of force deficit after lengthening contractions in soleus muscle from trained rats. J Appl Physiol, 88, 1254-8.

GROUNDS, M. D. (1998) Age-associated changes in the response of skeletal muscle cells to exercise and regeneration. Annals of the New York Academy of Sciences, 854, 78-91.

HAWKE, T. J. \& GARRY, D. J. (2001) Myogenic satellite cells: physiology to molecular biology. J Appl Physiol, 91, 534-551.

HESSELINK, M. K., KUIPERS, H., GEURTEN, P. \& VAN STRAATEN, H. (1996) Structural muscle damage and muscle strength after incremental number of isometric and forced lengthening contractions. J Muscle Res Cell Motil, 17, 335-41.

HOLLANDER, M.S., (In preparation 2007)

HORITA, T., KOMI, P. V., NICOL, C. \& KYROLAINEN, H. (1999) Effect of exhausting stretch-shortening cycle exercise on the time course of mechanical behaviour in the drop jump: possible role of muscle damage. Eur J Appl Physiol Occup Physiol, 79, 160-7. 
JACKSON, M. J., JONES, D. A. \& EDWARDS, R. H. (1984) Experimental skeletal muscle damage: the nature of the calcium-activated degenerative processes. Eur J Clin Invest, $14,369-74$.

JACKSON, M. J., PAPA, S., BOLANOS, J., BRUCKDORFER, R., CARLSEN, H., ELLIOT, R. M., FLIER, J., GRIFFITHS, H. R., HEALES, S., HOLST, B., LORUSSO, M., LUND, E., MOSKAUG, J. O., MOSER, U., DI PAOLA, M., POLIDORI, M. C., SIGNORILE, A., STAHL, W., VINA-RIBES, J. \& ASTLEY, S. B. (2002) Antioxidants, reactive oxygen and nitrogen species, gene induction and mitochondrial function. Mol Aspects Med, 23, 209-285.

JI, L. L. (1995) Oxidative stress during exercise: implication of antioxidant nutrients. Free Radic Biol Med, 18, 1079-1086.

JI, L. L., LEEUWENBURGH, C., LEICHTWEIS, S., GORE, M., FIEBIG, R., HOLLANDER, J. \& BEJMA, J. (1998) Oxidative stress and aging. Role of exercise and its influences on antioxidant systems. Ann NY Acad Sci, 854, 102-117.

JI, L. L. \& PETERSON, D. M. (2004) Aging, Exercise, and Phytochemicals Promises and Pitfalls. Ann N Y Acad Sci, 1019, 453-461.

JONES, D. A., NEWHAM, D. J., ROUND, J. M. \& TOLFREE, S. E. (1986) Experimental human muscle damage: morphological changes in relation to other indices of damage. $J$ Physiol, 375, 435-48.

KOH, T. J., PETERSON, J. M., PIZZA, F. X. \& BROOKS, S. V. (2003) Passive stretches protect skeletal muscle of adult and old mice from lengthening contraction-induced injury. J Gerontol A Biol Sci Med Sci, 58, 592-7.

KOMI, P. V. (2000) Stretch-shortening cycle: a powerful model to study normal and fatigued muscle. J Biomech, 33, 1197-206.

KOMULAINEN, J., KALLIOKOSKI, R., KOSKINEN, S. O., DROST, M. R., KUIPERS, H. \& HESSELINK, M. K. (2000) Controlled lengthening or shortening contraction-induced damage is followed by fiber hypertrophy in rat skeletal muscle. Int J Sports Med, 21, 107-12.

KOMULAINEN, J., TAKALA, T. E., KUIPERS, H. \& HESSELINK, M. K. (1998) The disruption of myofibre structures in rat skeletal muscle after forced lengthening contractions. Pflugers Arch, 436, 735-41. 
KRAJNAK, K., WAUGH, S., MILLER, R., BAKER, B., GERONILlA, K., ALWAY, S. E. \& CUTLIP, R. G. (2006) Proapoptotic factor bax is increased in satellite cells in the tibialis anterior muscles of old rats. Muscle Nerve, 24, 720-730.

LARSSON, L., GRIMBY, G. \& KARLSSON, J. (1979) Muscle strength and speed of movement in relation to age and muscle morphology. J Appl Physiol, 46, 451-456.

LEEUWENBURGH, C., FIEBIG, R., CHANDWANEY, R. \& JI, L. L. (1994) Aging and exercise training in skeletal muscle: responses of glutathione and antioxidant enzyme systems. Am J Physiol, 267, R439-45.

LEEUWENBURGH, C. \& JI, L. L. (1995) Glutathione Depletion in Rested and Exercised Mice: Biochemical Consequence and Adaptation. Arch Biochem Biophys, 316, 941-949.

LEVEILLE, S. G. (2004) Musculoskeletal aging. Curr Opin Rheumatol, 16, 114-8.

LIEBER, R. L., SCHMITZ, M. C., MISHRA, D. K. \& FRIDEN, J. (1994) Contractile and cellular remodeling in rabbit skeletal muscle after cyclic eccentric contractions. $J$ Appl Physiol, 77, 1926-34.

LIEBER, R. L., THORNELL, L. E. \& FRIDEN, J. (1996) Muscle cytoskeletal disruption occurs within the first 15 min of cyclic eccentric contraction. J Appl Physiol, 80, 278-84.

LIEBER, R. L., WOODBURN, T. M. \& FRIDEN, J. (1991) Muscle damage induced by eccentric contractions of 25\% strain. J Appl Physiol, 70, 2498-507.

MANFREDI, T. G., FIELDING, R. A., O'REILLY, K. P., MEREDITH, C. N., LEE, H. Y. \& EVANS, W. J. (1991) Plasma creatine kinase activity and exercise-induced muscle damage in older men. Med Sci Sports Exerc, 23, 1028-34.

MARTENSSON, J., MEISTER, A. (1989) Mitochondrial damage in muscle occurs after marked depletion of glutathione and is prevented by giving glutathione monoester. Proc Natl Acad Sci U S A, 86, 471-475.

MAURO, A. (1961) Satellite cell of skeletal muscle fibers. J Biophys Biochem Cytol, 9, 493-5.

MCARDLE, A., BROOME, C. S., KAYANI, A. C., TUlly, M. D., ClOSE, G. L., VASILAKI, A. \& JACKSON, M. J. (2006) HSF expression in skeletal muscle during myogenesis: Implications for failed regeneration in old mice. Exp Gerontol, 41, 497-500.

MCARDLE, A. \& JACKSON, M. J. (2000) Exercise, oxidative stress and ageing. J Anat, 197, 539-41. 
MCARDLE, A., PATTWELL, D., VASILAKI, A., GRIFFITHS, R. D. \& JACKSON, M. J. (2001) Contractile activity-induced oxidative stress: cellular origin and adaptive responses. Am J Physiol Cell Physiol, 280, 621-627.

MCARDLE, A., VAN DER MEULEN, J. H., CATAPANO, M., SYMONS, M. C., FAULKNER, J. A. \& JACKSON, M. J. (1999) Free radical activity following contraction-induced injury to the extensor digitorum longus muscles of rats. Free Radic Biol Med, 26, 1085-91.

MCARDLE, A., VASILAKI, A. \& JACKSON, M. J. (2002) Exercise and skeletal muscle ageing: cellular and molecular mechanisms. Ageing Research Reviews, 1, 79-93.

MCARDLE, F., SPIERS, S., ALDEMIR, H., VASILAKI, A., BEAVER, A., L., I., MCARDLE, A. \& JACKSON, M. J. (2004) Preconditioning of skeletal muscle against contractioninduced damage: the role of adaptations to oxidants in mice. J Physiol, 561, 233-244.

MCBRIDE, J. M., KRAEMER, W.J., TRIPLETT-MCBRIDE, T., SEBASTIANELLI, W. (1998) Effect of resistance exercise on free radical production. Med Sci Sports Exerc, 30, 67-72.

MCBRIDE, T. A., GORIN, F. A. \& CARLSEN, R. C. (1995) Prolonged recovery and reduced adaptation in aged rat muscle following eccentric exercise. Mech Ageing Dev, 83, 185200.

MCCORMICK, K. \& SCHULTZ, E. (1994) Role of satellite cells in altering myosin expression during avian skeletal muscle hypertrophy. Dev Dynamics, 199, 52-63.

MCCULLY, K. K. \& FAULKNER, J. A. (1985) Injury to skeletal muscle fibers of mice following lengthening contractions. J Appl Physiol, 59, 119-26.

MEISTER, A. (1991) Glutathione deficiency produced by inhibition of its synthesis, and its reversal; applications in research and therapy. Pharmac. Ther., 51.

MOSS, F. P. \& LEBLOND, C. P. (1970) Satellite cells as the source of nuclei in muscles of growing rats. Anat Rec, 170, 421-436.

PHELAN, J. N. \& GONYEA, W. J. (1997) Effect of radiation on satellite cell activity and protein expression in overloaded mammalian skeletal muscle. Anat Rec, 247, 179-188.

PORTER, M. M., VANDERVOORT, A. A. \& LEXELL, J. (1995) Aging of human muscle: structure, function, and adaptability. Scand J Med Sci Sports, 5, 129-142. 
POWERS, S. K., JI, L. L. \& LEEUWENBURGH, C. (1999) Exercise training-induced alterations in skeletal muscle antioxidant capacity: a brief review. Med Sci Sports Exerc, 31, 987-997.

RADAK, Z., CHUNG, H. Y. \& GOTO, S. (2005) Exercise and hormesis: oxidative stress-related adaptation for successful aging. Biogeront, 6, 71-75.

ROUBENOFF, R. (2001) Origins and clinical relevance of sarcopenia. Can J Appl Physiol, 26, 78-89.

ROY, R. R., TALMADGE, R. J., FOX, K., LEE, M., ISHIHARA, A. \& EDGERTON, V. R. (1997) Modulation of MHC isoforms in funtionally overloaded and exercised rat plantaris fibers. J Appl Physiol, 83, 280-290.

SACCO, P. \& JONES, D. A. (1992) The protective effect of damaging eccentric exercise against repeated bouts of exercise in the mouse tibialis anterior muscle. Exp Physiol, 77, 757-60.

SIU, P. M. \& ALWAY, S. E. (2005) Mitochondria-associated apoptotic signalling in denervated rat skeletal muscle. J Physiol, 565, 309-323.

SMITH, H. K., PLYLEY, M. J., RODGERS, C. D. \& MCKEE, N. H. (1999) Expression of developmental myosin and morphological characteristics in adult rat skeletal muscle following exercise-induced injury. Eur J Physiol, 80, 84-91.

STATISTICS, B. O. L. (1999) Civilian labor force 16 years and older by sex, age, race, and Hispanic origin, 1988, 1998, and projected 2008.

STAUBER, W. T. \& SMITH, C. A. (1998) Cellular responses in exertion-induced skeletal muscle injury. Mol Cell Biochem, 179, 189-96.

STUPKA, N., TARNOPOLSKY, M. A., YARDLEY, N. J. \& PHILLIPS, S. M. (2001) Cellular adaptation to repeated eccentric exercise-induced muscle damage. J Appl Physiol, 91, 1669-78.

UCHIYAMA, S., TSUKAMOTO, H., YOSHIMURA, S. \& TAMAKI, T. (2006) Relationship between oxidative stress in muscle tissue and weight-lifting-induced muscle damage. Eur J Physiol, 452, 109-116.

URSO, M. L. \& CLARKSON, P. M. (2003) Oxidative stress, exercise, and antioxidant supplementation. Toxicology, 189, 41-54.

VANDERVOORT, A. A. (2002) Aging of the human neuromuscular system. Muscle Nerve, 25, $17-25$. 
VIJAYAN, K., THOMPSON, J. L., NORENBERG, K. M., FITTS, R. H. \& RILEY, D. A. (2001) Fiber-type susceptibility to eccentric contraction-induced damage of hindlimbunloaded rat AL muscles. J Appl Physiol, 90, 770-6.

WARREN, G. L., HAYES, D. A., LOWE, D. A. \& ARMSTRONG, R. B. (1993) Mechanical factors in the initiation of eccentric contraction-induced injury in rat soleus muscle. $J$ Physiol, 464, 457-75.

WARREN, G. L., HAYES, D. A., LOWE, D. A., GUO, W. \& ARMSTRONG, R. B. (1991) Mechanical Factors in Exercise-Induced Muscle Injury. FASEB Journal, 5.

WARREN, G. L., INGALLS, C. P., SHAH, S. J. \& ARMSTRONG, R. B. (1999) Uncoupling of in vivo torque production from EMG in mouse muscles injured by eccentric contractions. J Physiol, 515 ( Pt 2), 609-19.

WEINDRUCH, R. (1995) Interventions based on the possibility that oxidative stress contributes to sarcopenia. J Gerontol A Biol Sci Med Sci, 50, 157-161.

WILLIAMS, P., WATT, P., BICIK, V.\& GOLDSPINK, G. (1986) Effect of stretch combined with electrical stimulation on the type of sarcomeres produced at the ends of muscle fibers. Exp Neurol, 93, 500-509.

WRETMAN, C., LIONIKAS, A., WIDEGREN, U., LANNERGREN, J., WESTERBLAD, H. \& HENRIKSSON, J. (2001) Effects of concentric and eccentric contractions on phosphorylation of MAPK $\mathrm{erk}^{\mathrm{e} / 2}$ and $\mathrm{MAPK}^{\mathrm{p} 38}$ in isolated rat skeletal muscle. $J$ Physiol, $535,155-164$.

ZERBA, E., KOMOROWSKI, T. E. \& FAULKNER, J. A. (1990) Free radical injury to skeletal muscles of young, adult, and old mice. Am J Physiol, 258, C429-35. 
Figure 1: In Vivo Rodent Dynamometer

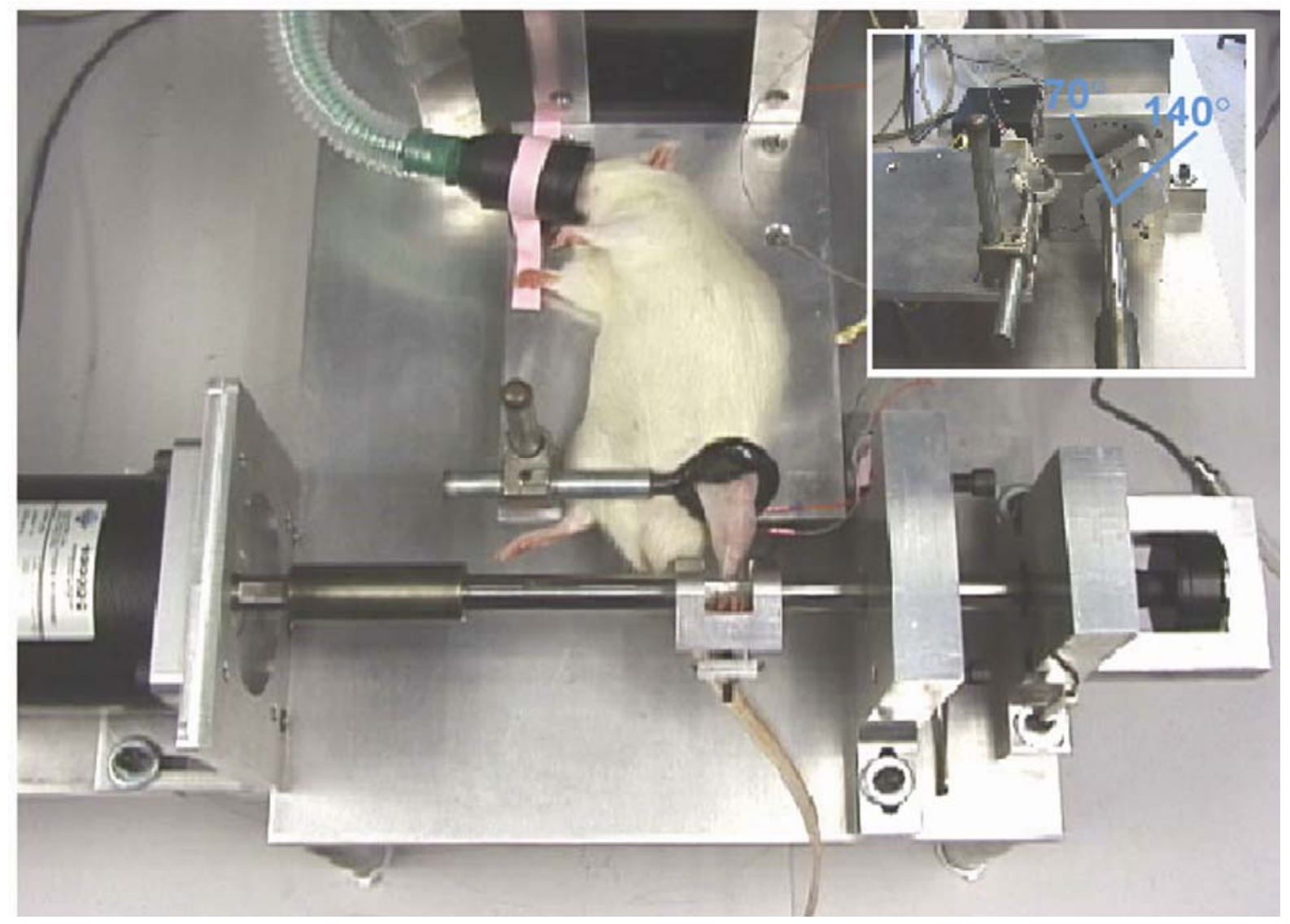


Figure 2: The Glutathione Pathway: GSH Inhibition via BSO

Glutathione deficiency

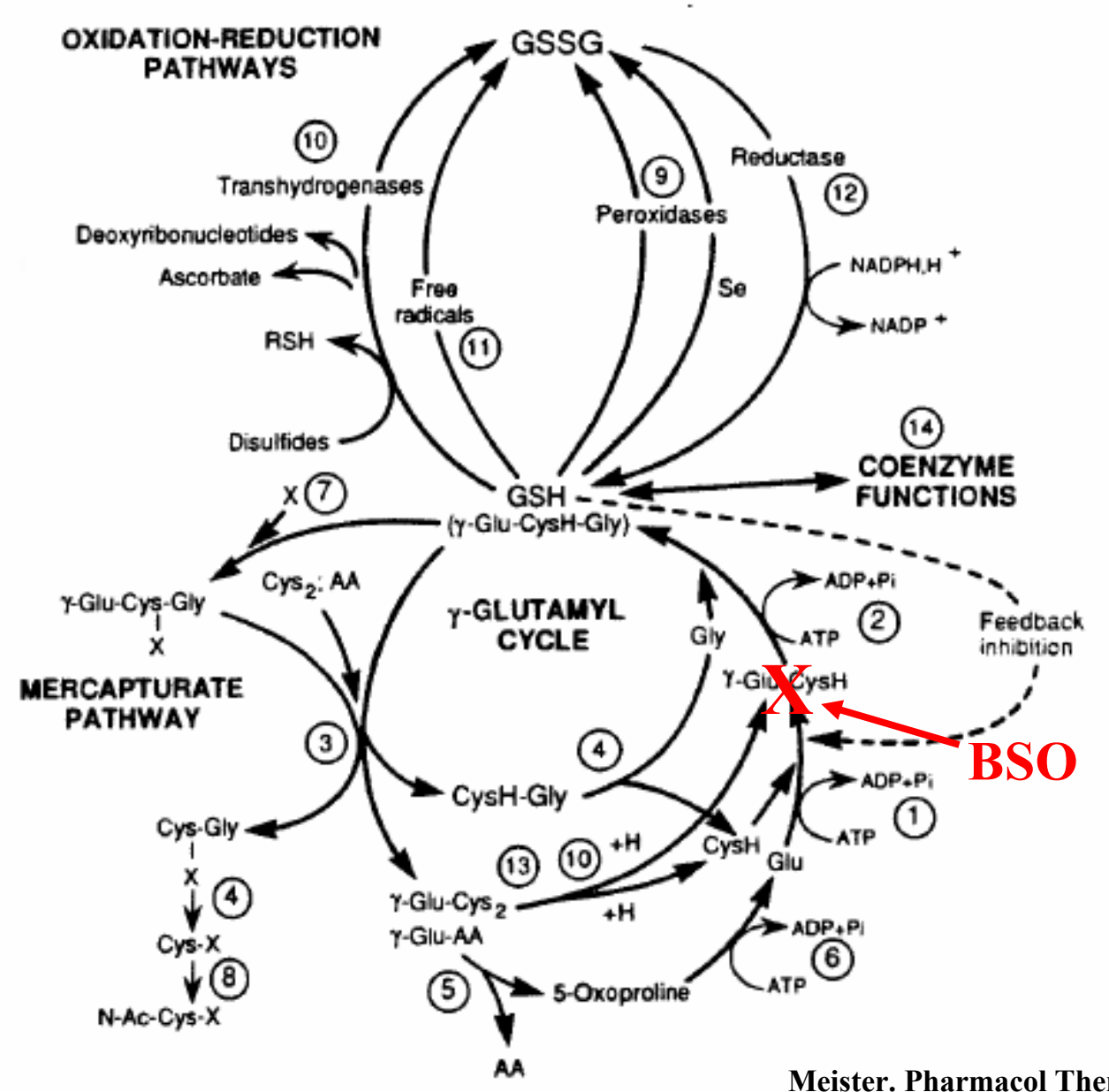

Meister. Pharmacol Ther. 1991 
Stereological Analysis of Muscle Morphology Following Exposure to Repetitive StretchShortening Cycles in a Rat Model.

Brent A. Baker, MS, ${ }^{1}$ Robert R. Mercer, ${ }^{1}$ Ken B. Geronilla, MS, ${ }^{1}$ Michael L. Kashon, PhD, ${ }^{1}$ G. R. Miller, BS, ${ }^{1}$ and Robert G. Cutlip, PhD ${ }^{1}$

${ }^{1}$ National Institute for Occupational Safety and Health (NIOSH), Health Effects Laboratory Division, Morgantown, WV, 26505, USA.

Address correspondence to:

Robert G. Cutlip, Ph.D.

National Institute for Occupational Safety and Health

Health Effects Laboratory Division

1095 Don Nehlen Drive

$\mathrm{M} / \mathrm{S} 2027$

Morgantown, WV 26505

Fax: (304) 285-6265; Tel: (304) 285-5968; E-mail: rgc8@,cdc.gov

Short title: Stretch Shortening Cycle Muscle Injury 


\section{ABSTRACT:}

Repetitive motion is one risk factor associated with contraction-induced muscle injury, which leads to skeletal muscle degeneration, inflammation, and dysfunction. Aim: Since current methods are unable to quantify the acute degenerative and inflammatory responses of muscle tissue concurrently, the purpose of this study was to quantify the temporal myofiber response following exposure to injurious stretch-shortening cycles using a standardized stereological technique. Methods: Functional testing was performed on the ankle dorsiflexor muscles of Sprague-Dawley rats in vivo. Rats were anesthetized and exposed to 15-sets of 10 stretchshortening cycles (SSCs). Control rats were exposed to 15-sets of single isometric contractions of the same stimulation duration. Changes in muscle morphometry were assessed at $0.5,24,48$, 72 , and 240 hours post-exposure to quantify the degree of myofiber degeneration and inflammation in the tibialis anterior muscle from each group. Results: There was an increase in the volume density and average thickness of degenerating myofibers over time in the muscle collected from rats exposed to stretch-shortening cycles $(\mathrm{p}<0.0001)$ that was significantly greater than in muscle exposed to isometric contractions at 24,48 and $72 \mathrm{~h}$ post- exposure ( $\mathrm{p}=$ $0.003)$. The volume density of degenerative myofibers was associated with functional deficits at $48 \mathrm{~h}$. Conclusion: Stereological quantification of degenerative myofibers and interstitial space changes were associated with functional defects at 48-72 $\mathrm{h}$ post SSC-induced injury, thus, demonstrating stereology is an accurate measure of SSC-induced skeletal muscle injury.

Abbreviations: AL, adductor longus; ANOVA, analysis of variance; CI, cellular interstitium; $\mathrm{CON}$, isometric-control group; EDL, extensor digitorum longus; H\&E, hematoxylin and eosin; $\mathrm{Hz}$, hertz; LSD, least significant difference; LTA, left tibialis anterior; $\mu \mathrm{m}$, microns; min, minutes; ms, milliseconds; NCI, non-cellular interstitium; OCT, optimum cutting temperature medium; rad, radians; s, seconds; SSC, stretch-shortening cycle group; TA, tibialis anterior; V, volts

$\underline{\text { Key Words: }}$ stereology; morphometry; myofiber degeneration; interstitial space; stretchshortening cycles

Correspondence to: Robert G. Cutlip; e-mail: rgc8@,cdc.gov 


\section{INTRODUCTION}

A variety of methods have been used to examine the physiological and cellular responses to contraction-induced muscle injury. Exposure to concentric (shortening) or isometric muscle actions does not normally produce muscle injury (Warren et al., 1993a, Warren et al., 1999b, Faulkner et al., 1995, Lieber et al., 1996). However, exposure to eccentric contractions has been shown to result in an isometric force deficit (Warren et al., 1999b), structural disruption at the cellular level, and cellular infiltrates as a result of an inflammatory response (Warren et al., 1999b, Lieber et al., 1996, Faulkner et al., 1989, Warren et al., 1993c, Friden et al., 1983b, McCully and Faulkner, 1985). Even though numerous models have been used to study the functional and biological mechanisms of contraction-induced muscle injury, there is a paucity of studies quantifying both skeletal muscle damage and inflammation after contraction-induced muscle injury (Koh et al., 2003, Geronilla et al., 2003). The ability to rapidly quantify both skeletal muscle degeneration and inflammation in the same tissue following an injurious exposure would yield further insight into the injury and repair process of skeletal muscle. It has been shown that exposure to injurious eccentric muscle actions results in disruption of the cellular membrane, loss of intermediate filaments and structural proteins, and the influx of extracellular proteins into the cell (Komulainen et al., 2000, Komulainen et al., 1998, Friden and Lieber, 1998, Lieber et al., 1994). Sarcomeric lesions, disorganized actin, and Z-disc streaming also result after injury (Stupka et al., 2001, Vijayan et al., 2001, Devor and Faulkner, 1999, Lieber et al., 1991). During the injury process, damaged cells lose apposition to neighboring cells, and there is evidence of cellular infiltrates such as neutrophils and macrophages entering the damaged cells (Koh et al., 2003, Devor and Faulkner, 1999). Days after injury, the regenerative process is initiated and central nuclei appear present (Bigard et al., 1997b, Hesselink 
et al., 1996). At this time the muscle demonstrates a mixture of both degenerative and regenerative processes.

Three distinct methods have been used to quantify muscle damage: 1) By the percent of injured fibers (Koh et al., 2003, Faulkner et al., 1989, Van Der Meulen et al., 1997), the area fraction of damaged fibers (McCully and Faulkner, 1986, Lieber and Friden, 1993), or the number of damaged fibers (Devor and Faulkner, 1999), 2) By the number or percentage of fibers affected by a change in the structural proteins (desmin, fibronectin, and dystrophin) indicative of damage, or by the number of fibers that have central nuclei, indicative of regeneration (11-14), and 3) Muscle ultrastructure disruption as classified by fibers with sarcomeric lesions (15-18).

However, most, if not all, have not established strict classification criteria for quantifying myofiber damage. Contraction-induced models have been used to study muscle damage in extensor digitorum longus (EDL) and tibialis anterior (TA) muscles in rats (Van Der Meulen et al., 1997), mice (Koh et al., 2003), and rabbits (Lieber and Friden, 1993). Classification criteria for damaged versus non-damaged fibers in these studies included myofiber swelling, degenerative changes such as pale or discontinuous staining of the cytoplasm, the presence of infiltrating inflammatory cells, and the state of cell nuclei (Devor and Faulkner, 1999, Koh et al., 2003, Van Der Meulen et al., 1997, McCully and Faulkner, 1986, Lieber and Friden, 1993, Faulkner et al., 1989). While these approaches were well suited for the overall assessment of muscle damage, they did not lend themselves to quantitative assessment of the myofiber injury present. In addition these methods did not address the interstitial space's responses and modifications to the induced injury.

Even though there are benefits to investigating muscle damage with the existing morphological procedures, there are clear and distinct limitations to the current approaches. 
Even though it is well recognized that degeneration occurs to the myofiber and that interstitial space components participate in both degeneration as well as the inflammatory process, neither the degeneration nor inflammatory components are fully understood. For this reason it was imperative to utilize a standardized technique that rapidly and concurrently was able to quantify degeneration and inflammation (Krajnak K, 2004). Thus, the purpose of the present study was to systematically quantify skeletal muscle degeneration and changes occurring to the interstitial space, which could be related to the changes in muscle performance. To accomplish this, we determined the morphological properties of normal and degenerative myofibers based on a rigorous set of definitions and quantified the corresponding changes in the myofiber and the interstitium using a standardized stereological method (Underwood, 1970). We hypothesized that indices of myofiber degeneration and inflammation as well as modifications observed in the interstitial space would increase temporally as a result of the SSC-induced muscle injury. In order to determine if these quantitative measures of muscle morphology paralleled corresponding changes in muscle physiology, measurements of muscle functional properties were made. For this purpose animals were tested via a custom-built dynamometer (Cutlip et al., 1997a), which was used to regulate muscle contraction and record muscle output parameters produced by electrical stimulation of rat dorsi flexor muscles in vivo.

\section{MATERIALS and METHODS}

Animals: Male Sprague-Dawley rats $(n=72)$ were used in the present study $(422 \pm$ $19 \mathrm{~g}, 12$ weeks of age). Rats were housed in an AAALAC approved animal quarters where the temperature and light/dark cycle (dark cycle from 7:00 AM to 7:00 PM) were held constant and food and water were provided ad libitum. All rats were exposed to a standardized experimental 
protocol that complied with the Guidelines for the Care and Use of Laboratory Animals, which was approved by the NIOSH Animal Care and Use Committee. Animals were randomly assigned to either an isometric-control group $(\mathrm{CON}, \mathrm{n}=36)$ or a stretch-shortening cycle group $(\mathrm{SSC}, \mathrm{n}=$ 36). Each group was then randomly subdivided into $0.5,6,24,48,72$, or 240 hours (h) recovery groups $(n=6)$. A sub-set of animals, which constituted the CON-group at 0.5 and $48 \mathrm{~h}$ and SSC-group at 0.5 and 48-h recovery, were reported in a previous study (Geronilla et al., 2003).

Experimental Setup: Animals were tested on a custom-built rodent dynamometer as previously described (Cutlip et al., 1997a). Rat tibialis anterior (TA) muscles were exposed to a SSC protocol as previously described by (Geronilla et al., 2003). Briefly, rats were anesthetized with isoflurane gas in an "induction" tank (Surgivet Anesco Inc.), placed supine on a heated x-y positioning table of the rodent dynamometer, with an anesthetic mask over the nose and mouth of the animal. The knee was secured with a knee holder, and the left foot was secured in the load cell fixture with the ankle axis (assumed to be between the medial and lateral malleoli aligned with the axis of rotation of the load cell fixture). Each animal was monitored during the procedure to maintain proper anesthetic depth and body temperature.

Functional Testing: Platinum stimulating electrodes (catalogue \# F-E2, Grass Medical Instruments, Quincy MA, USA) were placed subcutaneously to span the common peroneal nerve. Activation of the electrical stimulator resulted in muscle contraction of the dorsi flexor muscle group. Stimulator settings were optimized to maximize dorsi flexor contractile performance as previously described (Geronilla et al., 2003, Cutlip et al., 2004). Muscle stimulation for all protocols was conducted at $120 \mathrm{Hertz}(\mathrm{Hz})$ stimulation frequency, 0.2- 
milliseconds (ms) pulse duration, and 4 Volts $(\mathrm{V})$ in magnitude. The joint angle of the rat ankle was defined as the angle between the tibia and the plantar surface of the foot. The angular position of the load cell corresponded with the foot position. Vertical forces applied to an aluminum sleeve fitted over the dorsum of the foot were translated to a load cell transducer (Sensotec, Inc.) in the load cell fixture. The force produced by the dorsiflexor muscles was measured at the interface of the aluminum sleeve and the dorsum of the foot. An isometric contraction was performed at 1.57 radians (rad) ankle angle using $300 \mathrm{~ms}$ simulation duration. An isometric contraction was performed two minutes preceding (pre) and 2 minutes (min) following (post) either fifteen sets of ten stretch-shortening contractions (SSC group) or fifteen isometric contractions (CON group), and prior to sacrifice at $0.5,6,24,48,72$, or $240 \mathrm{~h}$. All animals were returned to their home cages in the animal quarters after their respective treatment protocol and remained there with access to food and water ad libitum until time of sacrifice. Animals were monitored by the NIOSH animal quarters staff after their treatment exposure and did not show any signs of distress.

Injury Protocol: The contraction-induced injury group (SSC group) was exposed to 15-sets of SSCs. The SSCs were performed by full activation of the dorsi flexor muscles for $100 \mathrm{~ms}$, and then moving the load cell fixture from $1.13 \mathrm{rad}$ to $2.00 \mathrm{rad}$ angular position at a velocity of 8.72 $\mathrm{rad} / \mathrm{s}$, in a reciprocal fashion, for 10 oscillations. After 10 oscillations, the load cell fixture was stopped at angular position $1.13 \mathrm{rad}$ and the dorsi flexor group was deactivated $300 \mathrm{~ms}$ later. The total stimulation time per set was 2.8 seconds (s). The 15 -sets of 10 oscillations were conducted at 1-min intervals. 
Isometric Control Protocol: The CON group was exposed to 15 isometric contractions at 1min intervals. During each contraction, dorsi flexor muscles were stimulated for $2.8 \mathrm{~s}$ at $1.57 \mathrm{rad}$ using the same stimulation parameters and duration as in the SSC group.

Histology: Following completion of the $2^{\text {nd }}$ post-test rats from both the CON group and SSC group were weighed, anesthetized with sodium pentobarbital (ip, $10 \mathrm{mg} / 100 \mathrm{~g}$ body weight) and exsanguinated at $0.5,6,24,48,72$, and $240 \mathrm{~h}$ after exposure. The left TA (LTA) muscle was dissected, cleaned, and weighed (See Table 1 for absolute and relative muscle wet weights). The midbelly region was cut from the muscle and mounted on cork, immersed in optimal cutting temperature medium (OCT), frozen in isopentane cooled with liquid nitrogen, and stored at $80^{\circ} \mathrm{C}$. The midbelly region was selected to obtain the maximum tissue sample. Transverse sections were cut at 12 microns $(\mu \mathrm{m})$, mounted on pre-coated microscope slides, air dried, and stained using a routine procedure with Harris H\&E. Permount was used to attach coverslips to microscope slides. Stereological analyses were evaluated on a Leica DMLB microscope.

Myofiber Definitions: Stereology was used to quantify the degree of myofiber degeneration, and the accompanying changes in the interstitial space in the TA muscle from each group. Myofibers were defined by the following criteria: Normal myofibers demonstrated: 1) Complete contact with adjacent myofibers, 2) A smooth outer membrane, and 3) No presence of internal inflammatory cells. Degenerative myofibers displayed: 1) A loss of contact with adjacent myofibers, 2) Presence of internal inflammatory cells, and 3) An outer membrane interdigitated with inflammatory cells (Figure 1). 
Stereology: Quantitative morphometric methods were used to measure the volume fraction, surface densities, and average thickness of normal myofibers, degenerative myofibers, and the interstitial space as previously described (Underwood, 1970) The interstitium was divided into the endomysium and the perimysium space, which included capillaries. Stereology was also used to quantify the degree of inflammation, which was quantified as either non-cellular interstitium (NCI) indicative of edema, or cellular interstitium (CI); CI consisted of all possible infiltrating cells such as, but not limited to, inflammatory, endothelial, and fibroblasts. Again, volume and surface density was measured using standard morphometric analyses (Underwood, 1970). A stage micrometer was used to identify the mid-point of the sample section. Point and intercept counts using a 121-point / 11-line overlay graticule (12.5 mm square with 100 divisions) at 40x magnification were taken at five equally spaced sites across the section. This process was repeated, $2 \mathrm{~mm}$ on either side of the mid-point of the section for a total of 1210- points and 110intercept lines per section. Volume density or the percent (\%) tissue volume (referred to as $\%$ volume from this point) was computed from the percentage of points over the tissue section to points over normal myofibers, degenerative myofibers, cellular interstitium (CI) and noncellular interstitium plus capillaries (NCI). Intercepts over the line overlay were counted for the perimeter of normal myofibers, degenerative myofibers, and interstitium to myofiber transitions. Points and intercepts over blood vessels greater than $25 \mu \mathrm{m}$ in diameter were excluded. Average thickness or average distance was computed from two times the ratio of volume to surface density according to standard morphometric analysis (Underwood, 1970).

Statistical Analysis: Statistical analyses were conducted using SAS Version 8 (SAS Institute, Cary, NC). Muscle weights of the left tibialis anterior and isometric force data (difference in 
isometric force generated between the pre-test and the tests during the recovery period) were analyzed with 2-way analyses of variance (ANOVA). Where appropriate, post-hoc comparisons were conducted using Fishers LSD tests. Stereological measurements of the volume and thickness of cellular and noncellular components were analyzed using 2-way (treatment $x$ time) ANOVAs. Post-hoc comparisons were made using Fishers LSD tests. Since the data was deemed as ordinal, a non-parametric correlation coefficient was implemented to assess the relationship between force and volume and thickness. Therefore to compare measurements of $\%$ force drop, \% volume degenerative myofiber, \% volume $\mathrm{NCI}$, and \% volume $\mathrm{CI}$ in CON-group and SSC-group animals, Spearman's Rho was used for analyses of data, and results were reported via the Spearman rank-order correlation coefficient $\left(r_{s}\right)$. One section per animal with 6 animals per group was evaluated and the results expressed as mean \pm S.E.M.

\section{$\underline{\text { RESULTS }}$}

Analyses of Isometric Force Deficits: The isometric force decrement between the pre- and post-tests in both the SSC and CON groups are shown in Figure 2. There was a significant interaction between experimental treatment and recovery time $(\mathrm{p}=0.0016)$. Comparisons at each time point show that the isometric force decrement is significantly greater for the SSC group than the CON group at 6, 24, 48 and $72 \mathrm{~h}$ (Figure 2). In both groups there was an initial force decrement, which persisted for at least 30 minutes. However, in the CON group, the force decrement rapidly approaches baseline values by $6 \mathrm{~h}$ (The zero time point is within the $95 \%$ confidence interval around the point estimate), while in the SSC group this does not occur until $240 \mathrm{~h}$. Total recovery from contraction-induced injury was observed at $240 \mathrm{~h}$, which was recognized by no difference in isometric post-test and pre-test forces (Figure 2). 
Analyses of TA muscle wet weights: Weights of the LTA muscles (normalized to body weight) are shown in Figure 3. There was a significant interaction between experimental treatment and recovery time $(\mathrm{p}=0.0007)$. Analyses revealed that at 24,48 and $72 \mathrm{~h}$, the SSC groups had significantly higher muscle weights than the CON groups $(\mathrm{p}<0.05)$. However, at $240 \mathrm{~h}$ this difference was reversed $(p=0.024)$, with the muscle weights in the CON group being higher than weights in the SSC group. There were no statistically significant differences in the body mass of treatment groups prior to the application of treatments $(p=0.6148)$, and thus these differences in LTA weights cannot be attributed simply to differences in body mass.

Analyses of Temporal TA muscle response: Tissue sections from the 72-h CON group showed normal myofiber morphometry (Figure 4a). In addition, the 240-h CON group also showed normal fiber morphometry (Figure 4b). All tissue sections from the 72-h SSC group showed evidence of extensive interstitial swelling of the perimysium and endomysium (NCI), and interstitial hypercellularity (CI), as well as degenerative myofibers (Figure 4c). The 240-h SSC group had numerous myofibers with central nuclei, which was indicative of a degenerative/regenerative process (Figure 4d).

Stereological Analyses of myofiber degeneration: Statistical analyses for measurements of degeneration showed that there were no degenerative myofibers present in muscle collected from animals in the CON groups. In contrast, there was an increase in the $\%$ volume and average thickness of degenerating myofibers over time in the muscle collected from rats exposed to the SSC protocol $(p<0.0001)$. Further analyses revealed that the $\%$ volume and average thickness of degenerating myofibers from muscle exposed to the SSC protocol was significantly increased 
above muscle exposed to isometric contractions at 24-, 48- and 72-h post-exposure (Figure $5 \&$ Figure 6), respectively; $(p=0.003)$. There were no degenerative myofibers seen in muscle at 240-h post-exposure.

Stereological analyses of inflammation: Stereological estimates of inflammation and changes that occurred in the interstitial space also were analyzed. The 2-way ANOVA analyzing the $\%$ volume of the NCI produced a treatment $\mathrm{x}$ time interaction $(\mathrm{p}=0.009)$. Further analyses revealed that the $\%$ volume of the NCI was higher in the SSC group at 48-h post-exposure than in the CON group (Figure 5; $<<0.0001$ ). This is consistent with the increased edema seen in injured tissue collected at this time point (Geronilla et al., 2003). Measuring the \% volume of the $\mathrm{CI}$ and the average thickness of the interstitial space quantified cellular inflammation. The 2way ANOVAs for these variables produced treatment $\mathrm{x}$ time interactions (volume: $\mathrm{p}<0.0001$; thickness: $\mathrm{p}<0.0001)$. Post-hoc comparisons revealed that the $\%$ volume of $\mathrm{CI}$ was higher in muscles exposed to the SSC protocol at 48-h $(\mathrm{p}<0.0001)$ and $72-\mathrm{h}(\mathrm{p}<0.0001)$ post-exposure than in CON-group muscles (Figure 5). The average thickness of the interstitial space also was higher in muscle exposed to the SSC protocol than muscle from the CON-group rats at 48-h (p $<$ $0.0001)$ and $72-\mathrm{h}(\mathrm{p}<0.0001)$ post- injury (Figure 6$)$. These data are consistent with the presence of swollen and necrotic myofibers in injured tissues at these time points (Geronilla et al., 2003).

Response Kinetics for Function versus \% Volume or Average Thickness: In TA muscles for which contractile and stereological analyses were performed temporally following exposure to SSC contractions, response kinetics were profiled for degeneration and inflammation, 
respectively (Figures $7 \mathrm{a}, \mathrm{b}$ and $8 \mathrm{a}-\mathrm{c}$ ). Figure $7 \mathrm{a}$ depicts the response kinetics for the $\%$ force drop versus the $\%$ degenerative myofiber volume. Since the $\%$ force drop curve was identical in all profiles, this kinetic profile was repeated in all figures. The $\%$ force drop was affected most significantly from $0.5 \mathrm{~h}-48 \mathrm{~h}$, but returned towards baseline by $240 \mathrm{~h}$, while the $\%$ degenerative-myofiber volume displayed a significant increase at $24 \mathrm{~h}$ and $72 \mathrm{~h}$ following SSC exposure. The $\%$ degenerative-myofiber volume returned towards baseline by $240 \mathrm{~h}$. In figure $7 \mathrm{~b}$ the response kinetics for $\%$ force drop versus the average degenerative thickness was illustrated. Once again we see a very modest increase at early time points $0 \mathrm{~h}-6 \mathrm{~h}$, but a significant increase in the average degenerative thickness $24 \mathrm{~h}-72 \mathrm{~h}$. However, at $240 \mathrm{~h}$ we do not see a return to baseline in the average degenerative thickness.

Figures $8 \mathrm{a}$ and $8 \mathrm{~b}$ illustrate the response kinetics for the $\%$ force drop compared to the $\%$ $\mathrm{NCI}$ and $\% \mathrm{CI}$ volume, respectively. The \% volume NCI displayed a significant increase at $48 \mathrm{~h}$. The $\%$ volume NCI remained elevated at $72 \mathrm{~h}$ and $240 \mathrm{~h}$. Figure $8 \mathrm{~b}$ illustrates that at the early time points, $0.5 \mathrm{~h}-24 \mathrm{~h}$, no significant changes in $\% \mathrm{CI}$ were observed. At $48 \mathrm{~h}$ and $72 \mathrm{~h} \mathrm{a}$ significant increase in the \% volume CI was observed, which as seen in the NCI, remained elevated at $240 \mathrm{~h}$. In figure $8 \mathrm{c}$ no change in the thickness of the interstitial space was detected at $0.5 \mathrm{~h}-24 \mathrm{~h}$, but a significant increase in the thickness of the interstitial space was observed at 48 $\mathrm{h}$ and $72 \mathrm{~h}$. By 240-h post-injury the thickness of the interstitial space had returned to subbaseline values. Correlations of Isometric Force Drop\%, Volume Density, and Average Thickness: In TA muscles for which contractile and stereological analyses were performed temporally following exposure to either SSC contractions or isometric contractions, correlations were present. A negative correlation was observed between $\%$ force drop and $\%$ degenerative myofiber volume in the SSC group at 48-h post-injury, $\mathrm{r}_{\mathrm{s}}=-0.77$ (illustrated in Fig. 7a). Also a 
negative correlation was observed between the $\%$ force drop and the $\%$ volume NCI of the SSC group at 72 -h post- injury, $r_{s}=-0.66$ (illustrated in Fig. 8 a). In addition, $\%$ force drop and $\%$ volume CI was positively correlated at 48 -h post-injury, $\mathrm{r}_{\mathrm{s}}=0.77$ (illustrated in Fig. $8 \mathrm{~b}$ ).

\section{DISCUSSION}

The major findings of this study were that levels of myofiber degeneration, inflammation, and related changes in the interstitial space were quantifiable utilizing this standardized stereological technique (Krajnak K, 2004). Additionally, we show degenerative myofibers and interstitial changes were associated with functional performance temporally. Specifically, our working hypothesis was supported, and our results were indicative of increased temporal indices of degeneration and inflammation, and these measures were associated with functional performance. These results are in agreement with data shown in prior investigations (McCully and Faulkner, 1986, McCully and Faulkner, 1985), yet the majority of previous studies (Devor and Faulkner, 1999, Van Der Meulen et al., 1997, Lieber and Friden, 1993) are limited by having reported the number, percent, or cross-sectional area of damaged fibers at single time points following exposure. Also, previous histological studies have failed to directly quantify myofiber degeneration and its relationship to evident functional deficits following contraction-induced exposure. While the ability to characterize early-phase muscle damage is a fundamental benefit of immunohistochemical techniques, a lack of agreement in the extent of muscle damage reported morphologically and the magnitude of force loss still exists (Komulainen et al., 2000, Komulainen et al., 1998, Friden and Lieber, 1998).

An important aspect in quantifying skeletal muscle's degenerative response is that the reported biological changes, although small, may be extremely significant functionally. Since 
we are comparing significant increases and/or decreases to a control group that is not being affected, changes in vivo should not be discounted because they do not compare well with in vitro or in situ values that have been reported. In contrast, the capacity to detect significant changes and associations with functional measures illustrates how highly sensitive this piece of biological evidence is and should not be disregarded. By establishing rigorous exclusion criteria for categorizing cellular normality, degeneration, and inflammation, we were able to objectively determine the histological status of the muscle temporally after an injurious SSC exposure.

It was essential to devise a rapid method in which the most naïve examiner would be proficient in establishing highly reproducible results and would be able to verify the results, since observer and sampling biases do yield varied results (Glaser, 2000). Further comparisons, which were based on a review of the literature by Warren and colleagues (Warren et al., 1999b) in which histological methods were used in previous studies, have shown the inability to overcome researcher/observer bias, or sampling error, thus relating the extent of muscle damage morphologically to functional deficit. This may be predominantly due to sampling methods and classification techniques. Without implementing stringent classification criteria used to quantify the myofiber's response to a given exposure, inadequacies often exist. Various instances arise where fibers appear to be histologically damaged, however there may be no noted functional impairment because the force deficit observed was due to fatigue only (Vijayan et al., 2001). As a result, disconnects appear when attempting to relate muscle damage incurred at the ultrastructural level to function, and in the majority of the cases the interstitium's involvement is not detailed (Stupka et al., 2001). Early, single time points utilized for histological/ morphological samples have been shown to be problematic and do not relate to the time-course observed for decrements in contractile properties. Consequently, the capacity to characterize 
ultrastructural events and objectively quantify and/or predict myofiber degeneration and functional decrements may be limited. In the current study the stereological measurements of myofiber degeneration and the concomitant changes observed with the interstitial space were highly consistent with that seen in general histological examination (Geronilla et al., 2003) but yielded quantitative results.

Although prior stereological methods have examined cellular structure and organization of skeletal muscle (Baranska, 1997), they have not attempted to directly quantify the degree of myofiber degeneration and inflammation in the tissue concurrently. Moreover, a concern arises at the ultrastructural level regarding the procedure used to identify the functional deficit. One study in particular conducted by Lieber and colleagues (Lieber et al., 1994), reported a significant negative correlation between maximum tetanic tension and the percentage of desminnegative fibers in the TA and EDL of rabbits. The histological and contractile performance results 1 day and 2 days following exposure to eccentric exercise were presented for a sub-set of muscles. They reported that infiltration of inflammatory cells was present, but did not attempt to characterize the influence that cellular infiltration had on tetanic tension.

In addition, there are a paucity of studies quantifying both myofiber degeneration and inflammation following contraction-induced muscle injury (Friden et al., 1983b), (McCully and Faulkner, 1985). Our ability to quantify myofiber degeneration and inflammatory processes directly and their relationship to the quantified functional decrements can yield temporal insight into the role myofiber degeneration and inflammation plays in repair and recovery. While some histological approaches and techniques have been termed "too insensitive" to demonstrate the ability to quantify or detect cellular infiltration as a result of the imposed injury (Faulkner et al., 1989), this stereological technique does so convincingly. 
The presence of degenerative (necrotic) myofibers was significant in the TA muscle 24-h through 72 -h post-injury. In addition, the $\%$ force drop showed a peak response at 6 -h postinjury and remained significantly elevated until $72 \mathrm{~h}$. The $\%$ force drop was associated with the $\%$ degenerative myofiber volume and the degenerative myofiber average thickness 48 -h postexposure for the SSC group. If the increased degenerative volume leads to an increased degenerative myofiber thickness, then degenerative thickness may serve a critical purpose in producing modifications in and to the interstitial space. Furthermore, by 240 -h post-exposure there was neither an isometric force deficit nor presence of degenerative myofibers in the SSC group, signifying that myofibers were fully recovered from SSC-induced injury at this time. Even though less than $1 \%$ of the degenerative myofiber volume existed at $240 \mathrm{~h}$, a non-return to baseline in the degenerative myofiber thickness was evident at $240 \mathrm{~h}$; hence the residual degenerative thickness may be an important determinant of adaptation to the interstitial space.

The importance of inflammation and its contribution in repair and regeneration of skeletal muscle have been considered previously (Tidball et al., 1995), although it's importance is not thoroughly understood. In this study, the NCI response was not evident at early time points, but $\%$ volume NCI was significant at 48 -h post-injury. Interestingly, the increase in $\%$ volume $\mathrm{NCI}$ was associated with a significant increase in the $\%$ force drop at $72 \mathrm{~h}$. Furthermore, the increase observed in \% volume $\mathrm{CI}$ at $48 \mathrm{~h}$ was also associated with the increase in \% force drop, yet as the isometric force started to recover (\% force drop decreased), the $\% \mathrm{CI}$ continued to significantly increase until 72-h post-injury. One explanation is the increase in \% volume $\mathrm{CI}$ at $48 \mathrm{~h}$ and $72 \mathrm{~h}$ may be indicative of an increase in cellular infiltrates, specifically macrophages ((Koh et al., 2003),(Lapointe et al., 2002)) but not neutrophil infiltration (Pizza, 2002), as indicated by the temporal increases observed in the CI. Even though no association was 
observed at $48 \mathrm{~h}$ for the $\%$ volume $\mathrm{NCI}$ and $\%$ force drop, or at $72 \mathrm{~h}$ for the $\%$ volume $\mathrm{CI}$ and $\%$ force drop, the interstitial space's average thickness increase was associated with the $\%$ force drop at both 48-h and 72-h post-injury. Furthermore it should be noted that an increased thickness of a degenerating myofiber (myofiber swelling and cellular infiltrates) would be expected due to the increased permeability of the myofiber to edema and cellular infiltrates; our results depict this event as there was an observed increase in the \% volume $\mathrm{NCI}$ at $48 \mathrm{~h}$ and the $\%$ volume CI at 48 and $72 \mathrm{~h}$. While the case may indeed be that degenerative myofiber thickness' influence on the interstitial space does not limit the resolution capacity of skeletal muscle, it is plausible that non-cellular and cellular interstitium directly influences the interstitial space long-term, and this contributes extensively to the modifications to influence surviving myofibers. Thus, we propose that the non-cellular and cellular interstitium is influential in regulating the interstitial space's architecture; and, that CI influences on the interstitial space may be a highly sensitive measure for quantifying skeletal muscle's degenerative response. Further investigation is needed to determine the physiological contribution of CI (e.g. specific cell populations influential in regulating the interstitial space and myofiber's response - cell-cell interactions) and interstitial thickness has on SSC-induced injury in additional experimental groups (e.g. aged populations, dietary supplemented populations).

A limitation of the current study, which may be acknowledged for all investigative purposes, is that the results may be relative when comparisons and conclusions are made between species. Thus, we have demonstrated that this standardized stereological technique can rapidly assess, and more importantly quantify, myofiber degeneration, inflammation, and the associated changes occurring in and to the interstitial space temporally in muscle tissue morphologically after exposure to injurious SSCs. Further, these quantitative measurements of 
myofiber degeneration and inflammation are associated with isometric force deficits observed 24 h through $72 \mathrm{~h}$ post-injury.

\section{ACKNOWLEDGEMENTS}

The authors would like to thank Dr. Ann Hubbs and Dr. Petia Simeonova of the National

Institute for Occupational Safety and Health (NIOSH) and Dr. Stephen Alway of West Virginia University School of Medicine for their critical review and comments regarding this manuscript. 


\section{$\underline{\text { References: }}$}

Baranska (1997) Experimental and Molecular Pathology, 64, 13-21.

Bigard, A. X., Merino, D., Lienhard, F., Serrurier, B. and Guezennec, C. Y. (1997) Eur J Appl Physiol Occup Physiol, 75, 380-7.

Cutlip, R. G., Geronilla, K. B., Baker, B. A., Kashon, M. L., Miller, G. R. and Schopper, A. W. (2004) J Appl Physiol, 96, 507-16.

Cutlip, R. G., Stauber, W. T., Willison, R. H., McIntosh, T. A. and Means, K. H. (1997) Medical \& Biological Engineering \& Computing, 35, 540-3.

Devor, S. T. and Faulkner, J. A. (1999) J Appl Physiol, 87, 750-6.

Faulkner, J. A., Brooks, S. V. and Zerba, E. (1995) J Gerontol A Biol Sci Med Sci, 50 Spec No, 124-9.

Faulkner, J. A., Jones, D. A. and Round, J. M. (1989) Q J Exp Physiol, 74, 661-70.

Friden, J. and Lieber, R. L. (1998) Cell \& Tissue Research, 293, 165-71.

Friden, J., Sjostrom, M. and Ekblom, B. (1983) Int J Sports Med, 4, 170-6.

Geronilla, K. B., Miller, G. R., Mowrey, K. F., Wu, J. Z., Kashon, M. L., Brumbaugh, K., Reynolds, J., Hubbs, A. and Cutlip, R. G. (2003) Eur J Appl Physiol, 90, 144-53.

Glaser (2000) Journal of Chemical Neuroanatomy, 20, 115-126.

Hesselink, M. K., Kuipers, H., Geurten, P. and Van Straaten, H. (1996) J Muscle Res Cell Motil, 17, 335-41.

Koh, T. J., Peterson, J. M., Pizza, F. X. and Brooks, S. V. (2003) J Gerontol A Biol Sci Med Sci, 58, 592-7.

Komulainen, J., Kalliokoski, R., Koskinen, S. O., Drost, M. R., Kuipers, H. and Hesselink, M. K. (2000) Int J Sports Med, 21, 107-12. 
Komulainen, J., Takala, T. E., Kuipers, H. and Hesselink, M. K. (1998) Pflugers Arch, 436, 73541.

Krajnak K, M. R., Baker BA, Geronilla KB, Miller GR, Cutlip RG (2004), Vol. 36(5) Medicine and Science in Sports and Exercise, Indianapolis, IN, pp. \#1831.

Lapointe, B. M., Frenette, J. and Cote, C. H. (2002) J Appl Physiol, 92, 1995-2004.

Lieber, R. L. and Friden, J. (1993) J Appl Physiol, 74, 520-6.

Lieber, R. L., Schmitz, M. C., Mishra, D. K. and Friden, J. (1994) J Appl Physiol, 77, 1926-34.

Lieber, R. L., Thornell, L. E. and Friden, J. (1996) J Appl Physiol, 80, 278-84.

Lieber, R. L., Woodburn, T. M. and Friden, J. (1991) J Appl Physiol, 70, 2498-507.

McCully, K. K. and Faulkner, J. A. (1985) J Appl Physiol, 59, 119-26.

McCully, K. K. and Faulkner, J. A. (1986) J Appl Physiol, 61, 293-9.

Pizza, F. X. (2002) Journal of Applied Physiology, 92, 1873-1878.

Stupka, N., Tarnopolsky, M. A., Yardley, N. J. and Phillips, S. M. (2001) J Appl Physiol, 91, 1669-78.

Tidball, J. G., Albrecht, D. E., Lokensgard, B. E. and Spencer, M. J. (1995) J Cell Sci, 108 ( Pt 6), 2197-204.

Underwood, E. E. (1970) Quantatative Stereology, Addison-Wesley Publishing Co, Reading, MA.

Van Der Meulen, J. H., McArdle, A., Jackson, M. J. and Faulkner, J. A. (1997) J Appl Physiol, 83, 817-23.

Vijayan, K., Thompson, J. L., Norenberg, K. M., Fitts, R. H. and Riley, D. A. (2001) J Appl Physiol, 90, 770-6. 
Warren, G. L., Hayes, D. A., Lowe, D. A. and Armstrong, R. B. (1993a) J Physiol, 464, 457-75.

Warren, G. L., Lowe, D. A. and Armstrong, R. B. (1999) Sports Medicine, 27, 43-59.

Warren, G. L., Lowe, D. A., Hayes, D. A., Karwoski, C. J., Prior, B. M. and Armstrong, R. B. (1993b) J Physiol, 468, 487-99. 


\section{TABLE CAPTION}

\section{Table 1.}

$\underline{\text { Absolute and Relative Left Tibialis Anterior Muscle Weights. }}$

The left tibialis anterior absolute and relative muscle weights are shown as mean values +/- s.e.m. Each row signifies the respective muscle wet weights at the specified sacrifice time point. The upper panel of the table represents the isometric-control $(\mathrm{CON})$, while the lower panel of the table represents the stretch-shortening cycle (SSC) group.

\section{FIGURE CAPTIONS}

\section{Figure 1.}

Examples of Myofiber Definitions.

The top three light micrographs illustrate the typical close apposition and smooth membrane profiles of normal myofibers (arrows). The three lower profiles illustrate the loss of contact with neighboring myofibers (arrows), which was one criterion used to define degenerative myofiber profiles. As illustrated, these myofibers also displayed a highly ruffled outer membrane, which was interdigitated with inflammatory cells.

\section{Figure 2.}

Isometric force changes in the isometric-control groups (CON) and stretch-shortening groups (SSC) after injury.

The effect of exposure to SSCs on change in isometric forces generated by the dorsi flexors from 0.5 hours to 240 hours after the exposure was significantly different than those forces resulting 
from exposure to isometric contractions $(*$ indicates significant differences, $\mathrm{p}<0.0016)$. Data shown are mean values $+/$ - s.e.m.

\section{Figure 3.}

Wet weights of the left tibialis anterior muscle (standardized to $100 \mathrm{~g}$ body weight) in animals exposed to isometric-control (CON) or stretch-shortening cycle (SSC) protocols.

There was a significant interaction between experimental treatment and recovery time on left tibialis anterior muscle weights ( $\mathrm{p}=0.0007)$. At 24,48 and $72 \mathrm{~h}$ the SSC groups had significantly higher muscle weights than did the CON groups, while at 240 hours this difference was reversed. Data shown are mean values $+/$ - s.e.m (* indicates significantly different from CON groups relative to the same time points, $\mathrm{p}<0.05)$

\section{Figure 4.}

Morphological changes in muscle tissue after exposure to isometric or stretch-shortening protocols.

Tissue sections from the 72-hour isometric-control group showed normal myofiber arrangement (Figure 4a). The 240-hour isometric-control group also showed normal fiber arrangement (Figure 4b). All tissue sections from the 72-hour SSC-exposed group showed extensive interstitial swelling of the perimysium and endomysium NCI (arrow - NCI), swollen myofibers, interstitial hypercellularity due to CI (arrow - CI), and degenerative myofibers (asterisk). (Figure 4c). The 240-hour SSC-exposed group had numerous 
fibers with central nuclei (arrow - CN) and virtually no remaining degenerative fibers (Figure 4d). The scale shown is $100 \mu \mathrm{m}$.

\section{Figure 5.}

The volume of cellular and non-cellular components of muscle exposed to isometric (CON) or stretch-shortening cycle (SSC) contractions. Isometric contractions $(\mathrm{CON})$ did not significantly alter the interstitial space or produce the formation of degenerative myofibers. In contrast, exposure to SSCs resulted in increased volume of tissue occupied by degenerative myofibers (Deg. Myo), CI and $\mathrm{NCI}(*$ indicates significantly greater than isometric-control group muscles relative to $\mathrm{t}$ he same time point, $(\mathrm{p}<0.01)$.

\section{Figure 6.}

The thickness of degenerative myofibers and the interstitial space of muscle exposed to Isometric (CON) or stretch-shortening cycle (SSC) contractions.

The thickness of degenerative myofibers and the interstitial space response after exposure to stretch shortening contractions $(*$ indicates significantly greater than isometric control group relative to the same time point, $\mathrm{p}<0.01$ ). These variables are not affected by exposure to isometric contractions. 


\section{Figure 7 (a and b).}

a. Response kinetics for the \% force drop versus \% volume of degenerative myofibers for SSCexposed groups. Circled variables and asterisk illustrate observed correlation between \% force drop and \% volume of degenerative myofibers.

b. Response kinetics for the $\%$ force drop versus the average thickness of degenerative myofibers for SSC-exposed groups.

\section{Figure 8 (alb, and c).}

a. Response kinetics for the \% force drop versus \% volume of NCI for SSC-exposed groups. Circled variables and asterisk illustrate observed correlation between $\%$ force drop and \% volume of NCI.

b. Response kinetics for the \% force drop versus \% volume of CI for SSC-exposed groups. Circled variables and asterisk illustrate observed correlation between $\%$ force drop and \% volume of CI.

c. Response kinetics for the $\%$ force drop versus average thickness of the interstitial space for SSC-exposed groups. 
Table 1

\begin{tabular}{|l|l|l|}
\hline CON Group (Recovery -h) & $\begin{array}{l}\text { LTA Absolute Muscle } \\
\text { Wt. (mg) }\end{array}$ & $\begin{array}{l}\text { LTA Relative Muscle Wt. } \\
\text { (mg/100g body weight) }\end{array}$ \\
\hline 0.5 & $831 \pm 37.0$ & $196 \pm 7.8$ \\
\hline 6 & $864 \pm 19.3$ & $202 \pm 3.1$ \\
\hline 24 & $864 \pm 22.2$ & $202 \pm 6.6$ \\
\hline 48 & $797 \pm 15.8$ & $193 \pm 5.3$ \\
\hline 72 & $792 \pm 14.0$ & $187 \pm 0.9$ \\
\hline 240 & $899 \pm 33.7$ & $202 \pm 5.8$ \\
\hline & & \\
\hline SSC Group (Recovery - h) & $\begin{array}{l}\text { LTA Absolute Muscle } \\
\text { Wt. (mg) }\end{array}$ & $\begin{array}{l}\text { LTA Relative Muscle Wt. } \\
\text { (mg/100g body weight) }\end{array}$ \\
\hline 0.5 & $819 \pm 23.9$ & $197.2 \pm 5.8$ \\
\hline 6 & $907.3 \pm 47.9$ & $212 \pm 4.9$ \\
\hline 24 & $938 \pm 21.1$ & $220 \pm 2.3$ \\
\hline 48 & $963 \pm 23.5$ & $229 \pm 8.5$ \\
\hline 72 & $870 \pm 20.8$ & $205 \pm 7.1$ \\
\hline 240 & $801 \pm 41.7$ & $184 \pm 7.0$ \\
\hline
\end{tabular}


Figure 1
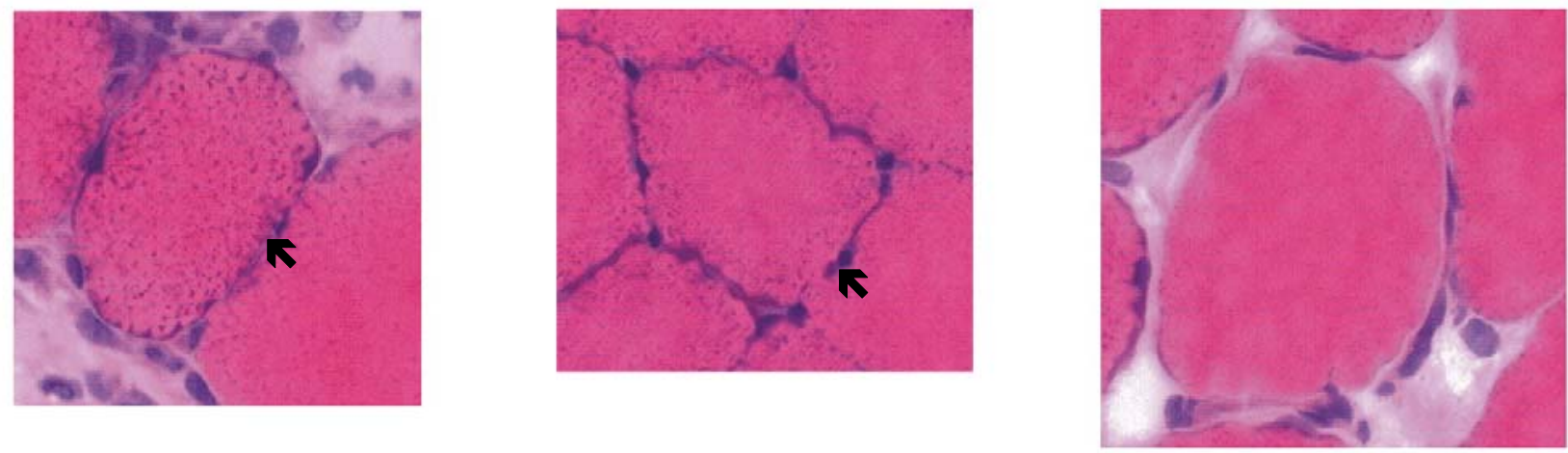

Normal Myofibers
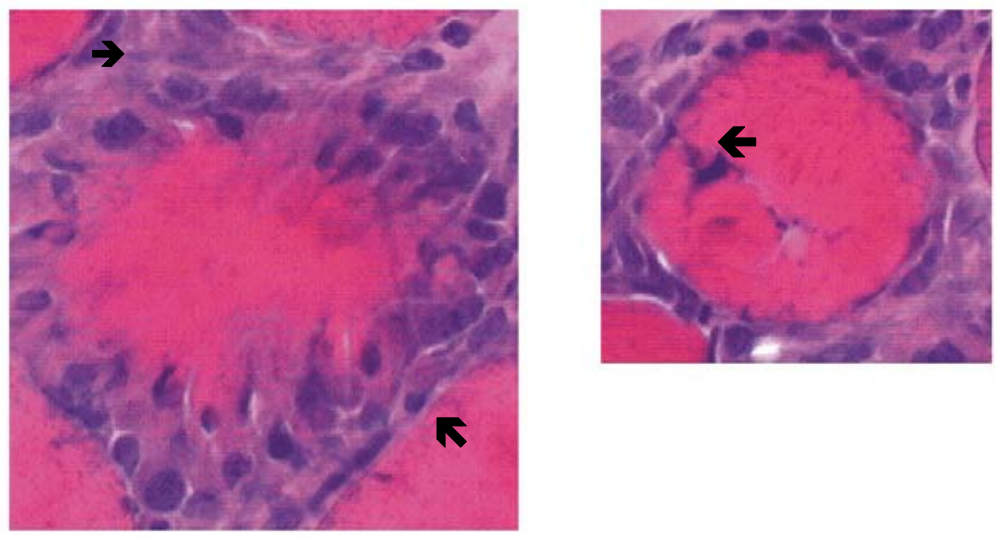

Degenerative Myofibers

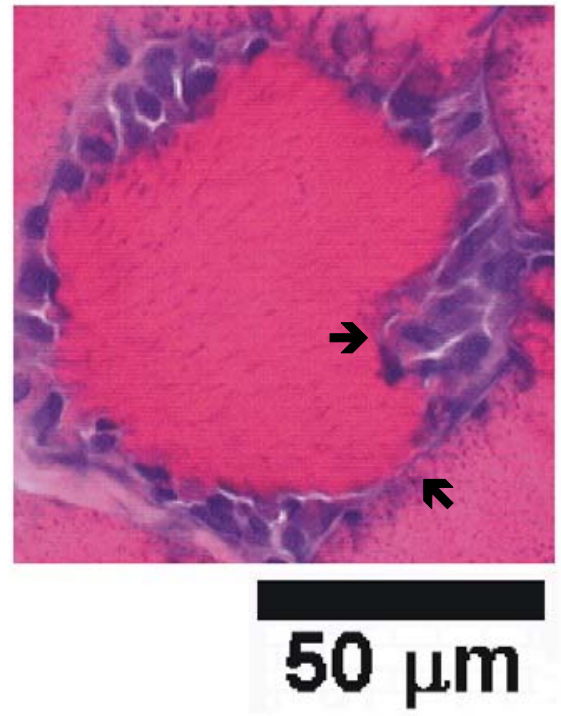


Figure 2

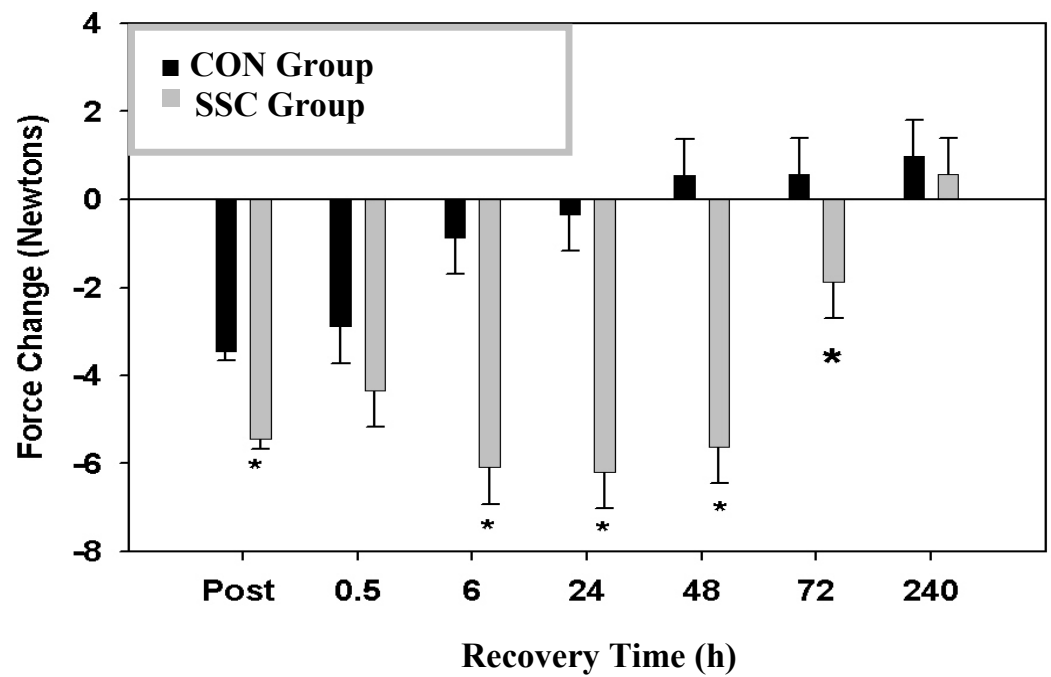


Figure 3

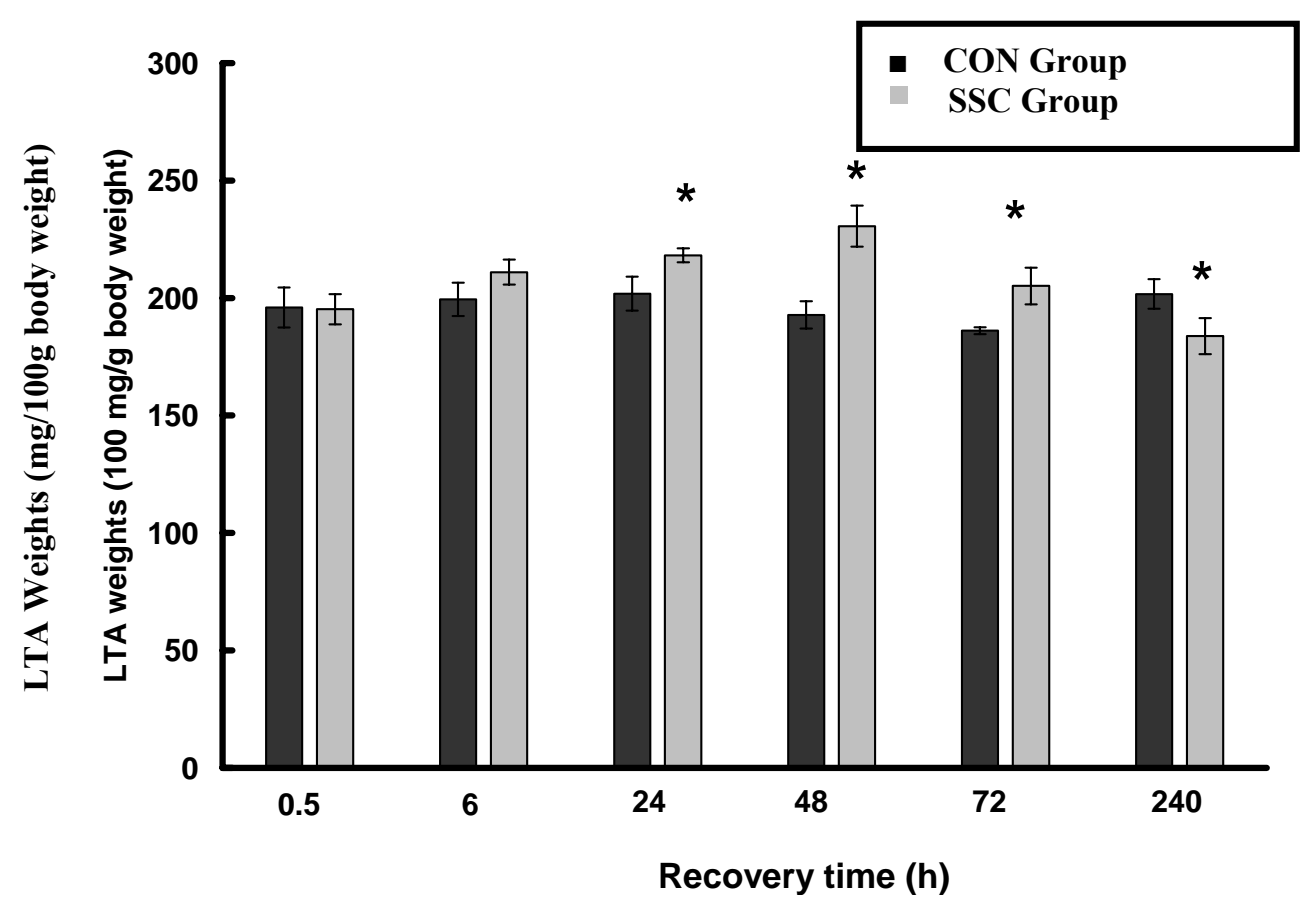


Figure 4

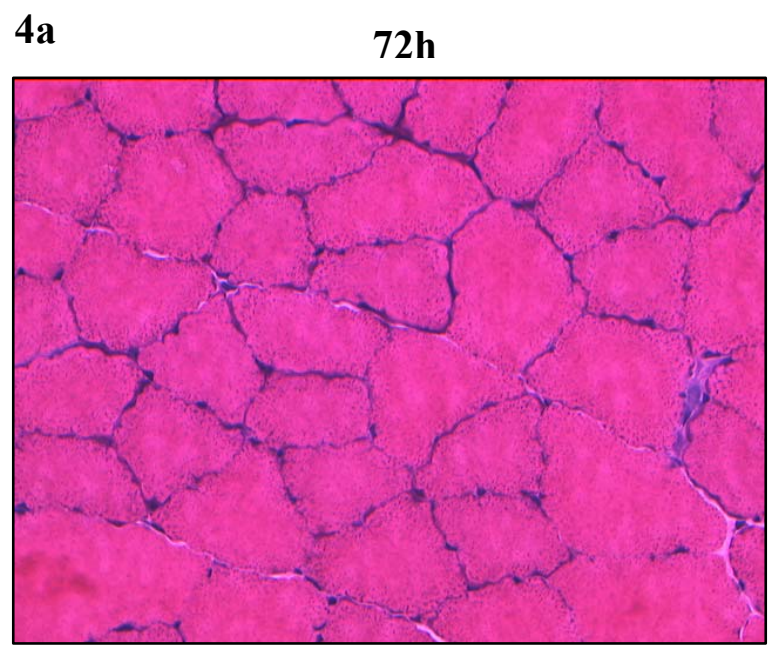

4b

240h

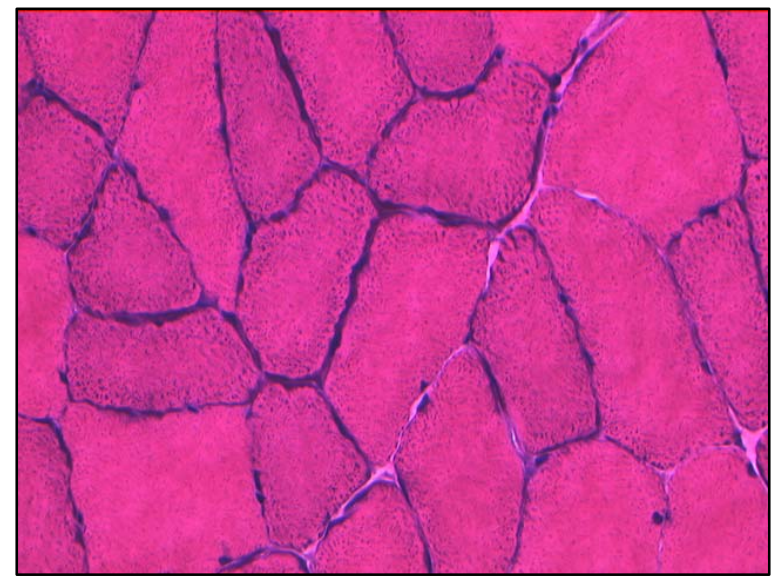

$4 c$

4d
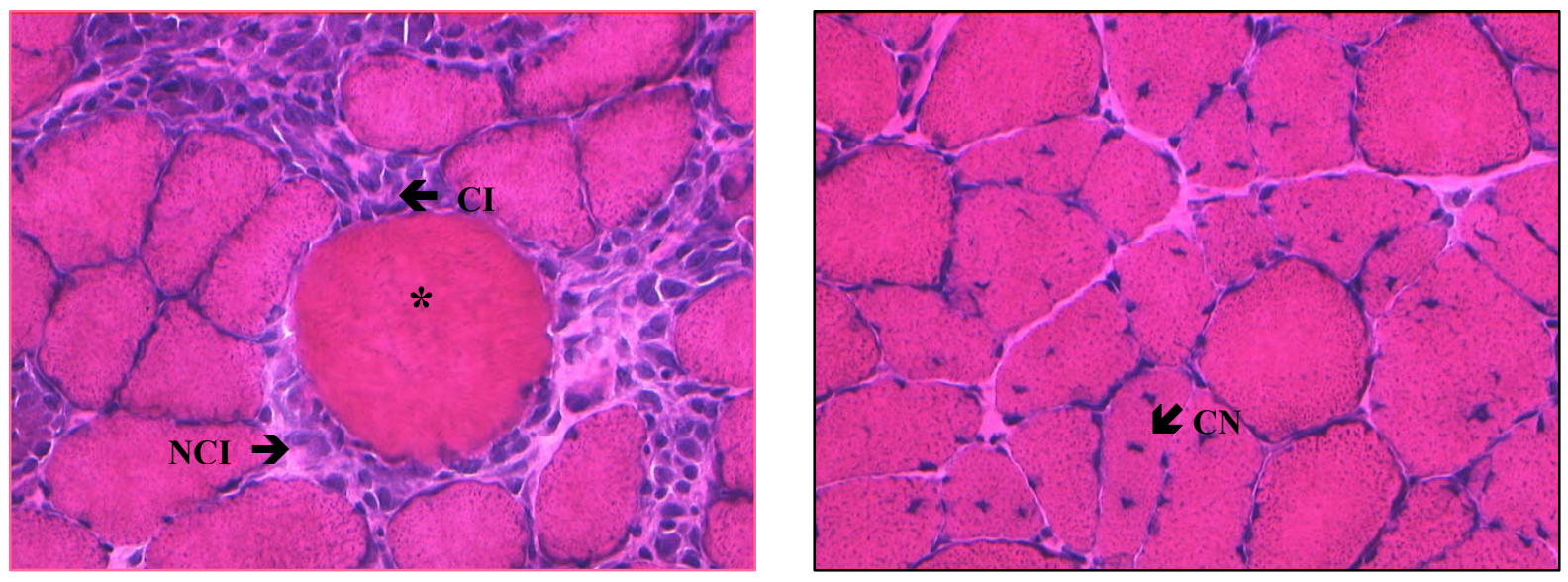
Figure 5

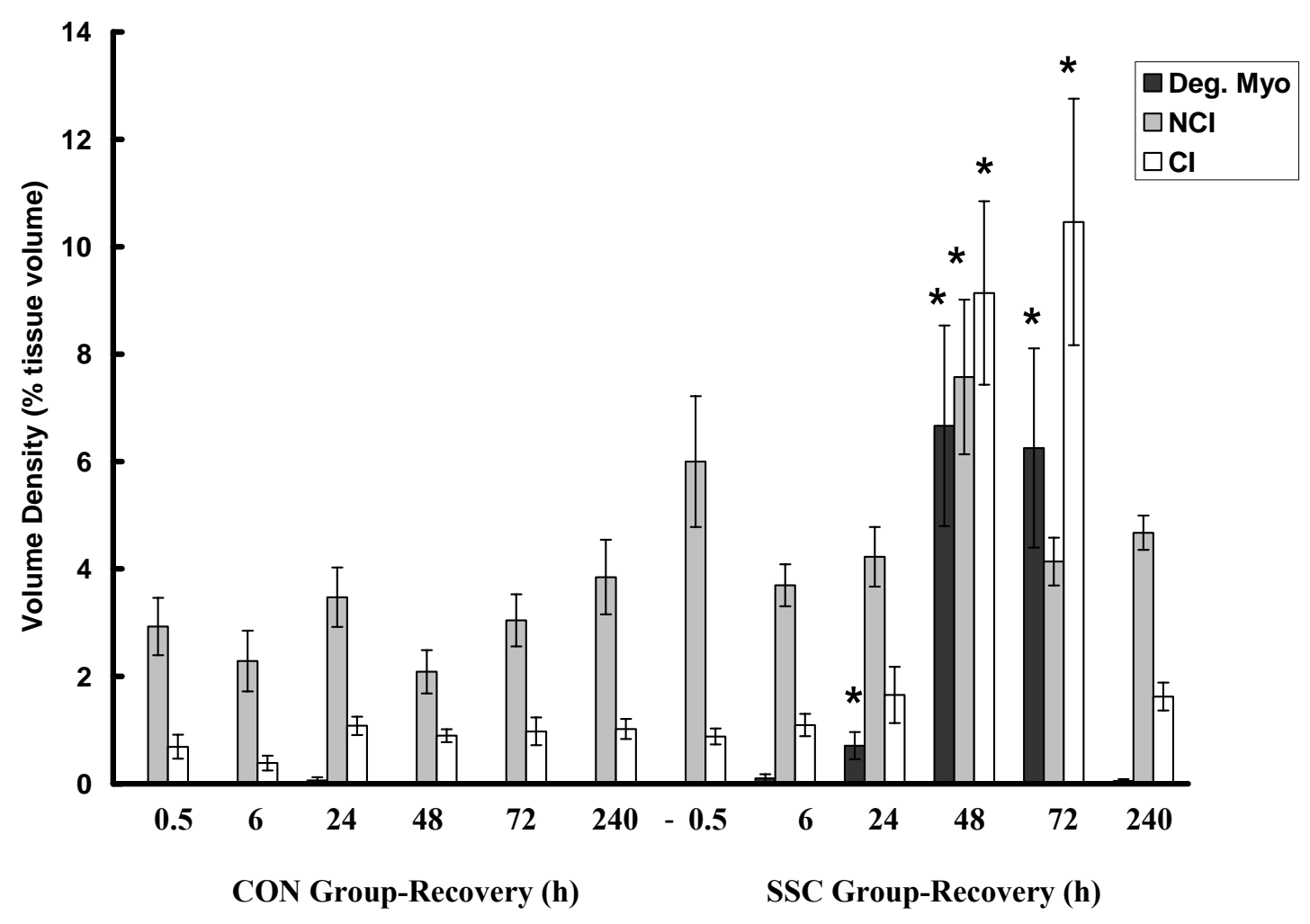


Figure 6

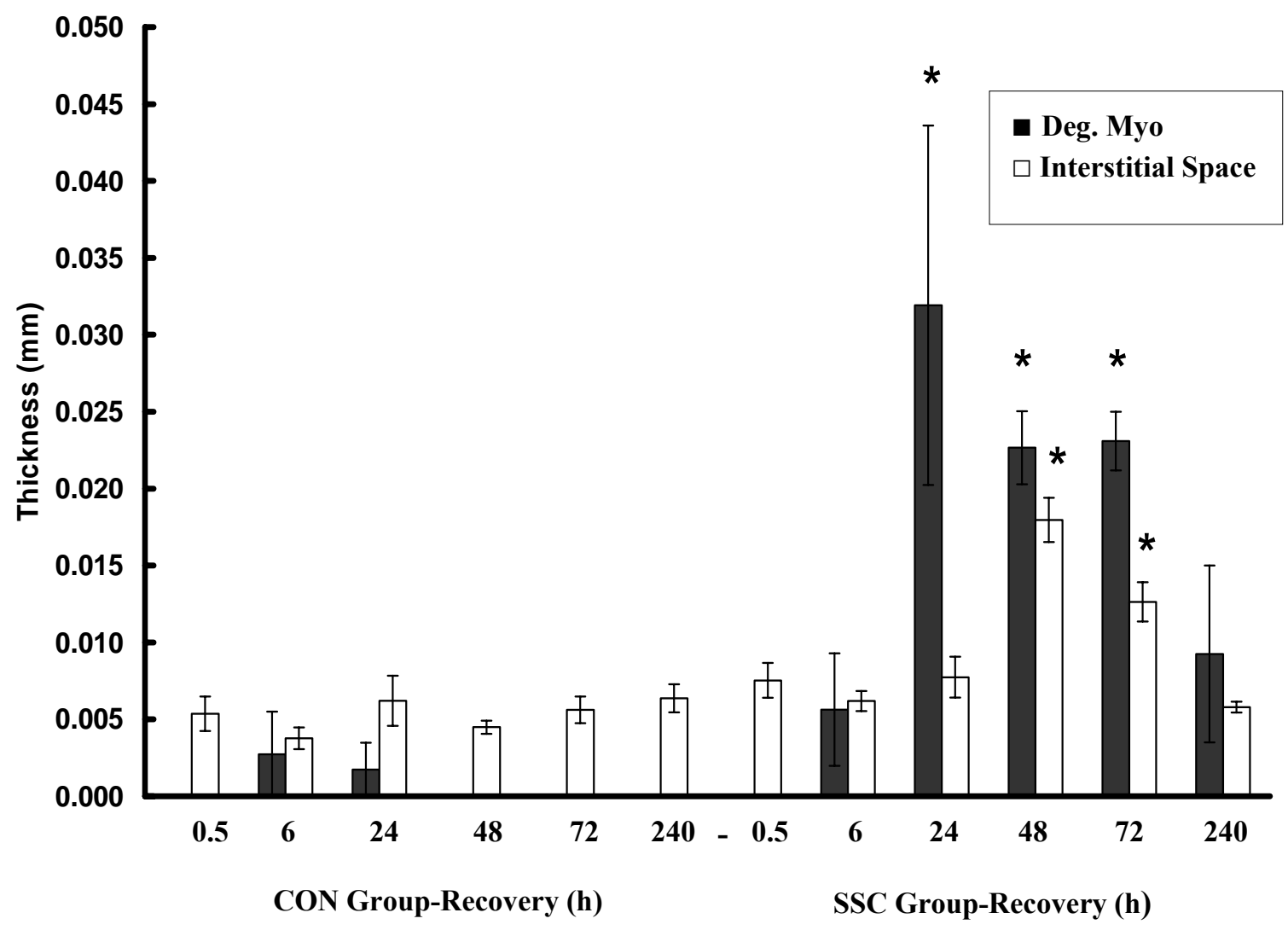




\section{Figure 7a}

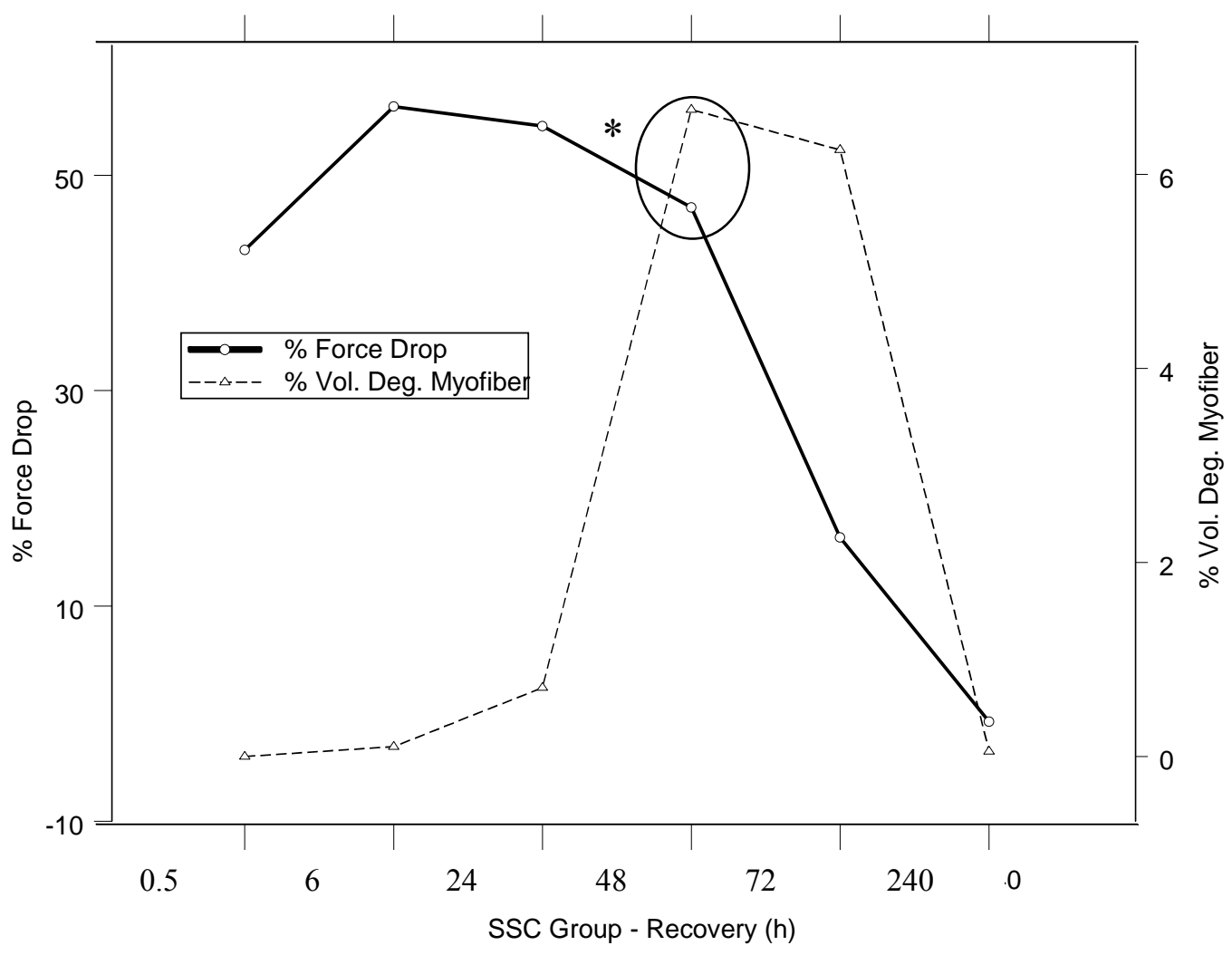


Figure 7b

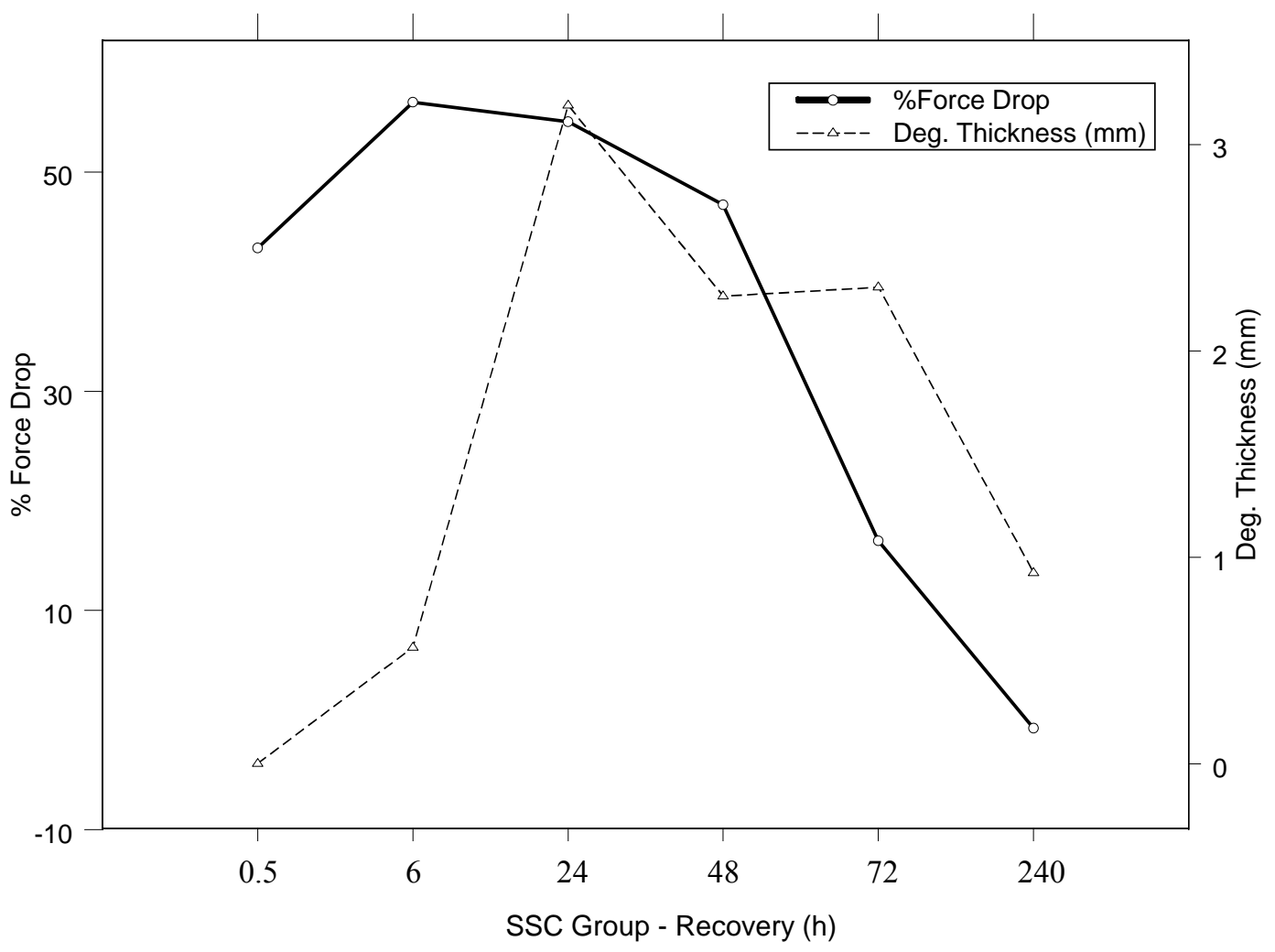


Figure 8a

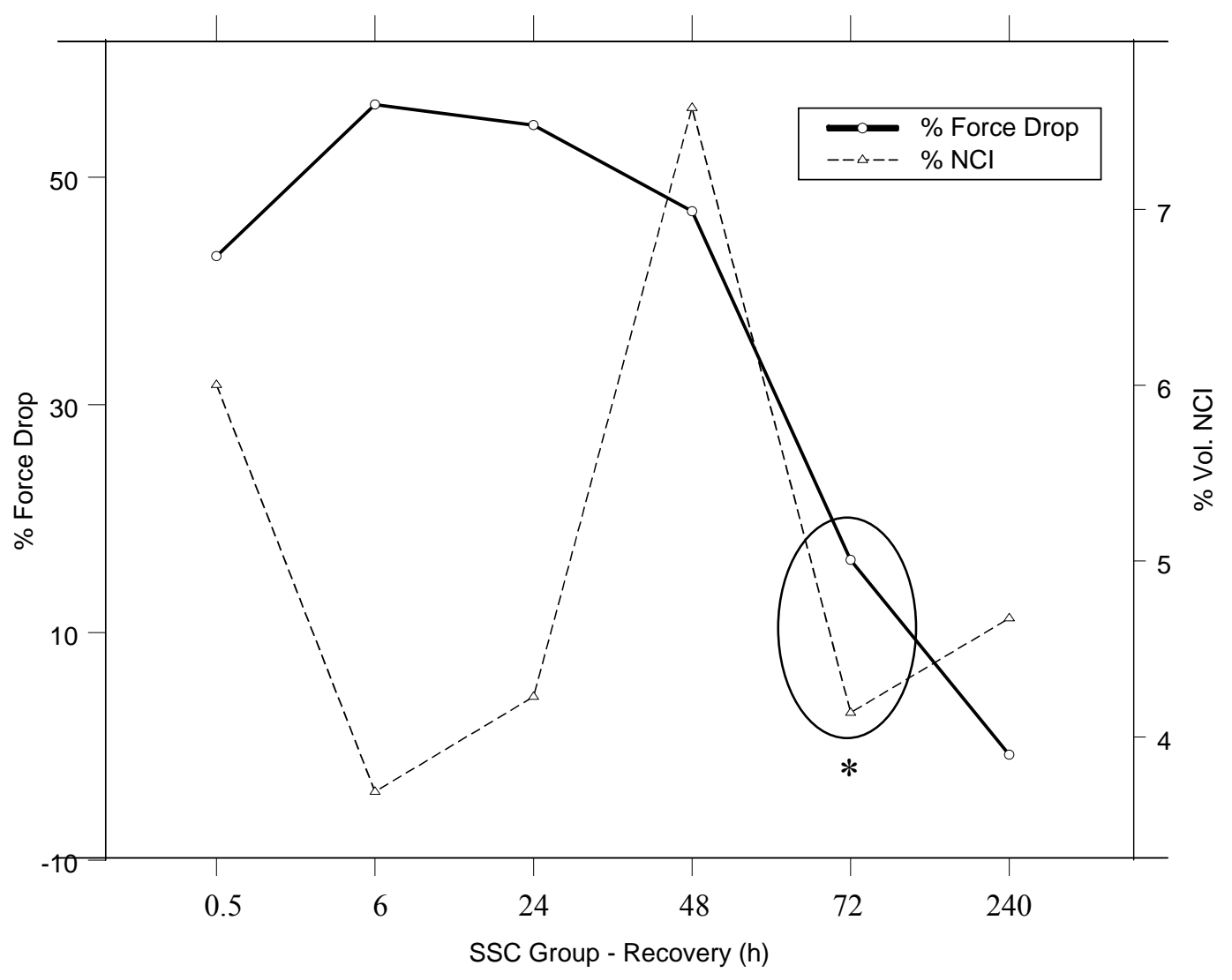


Figure 8b

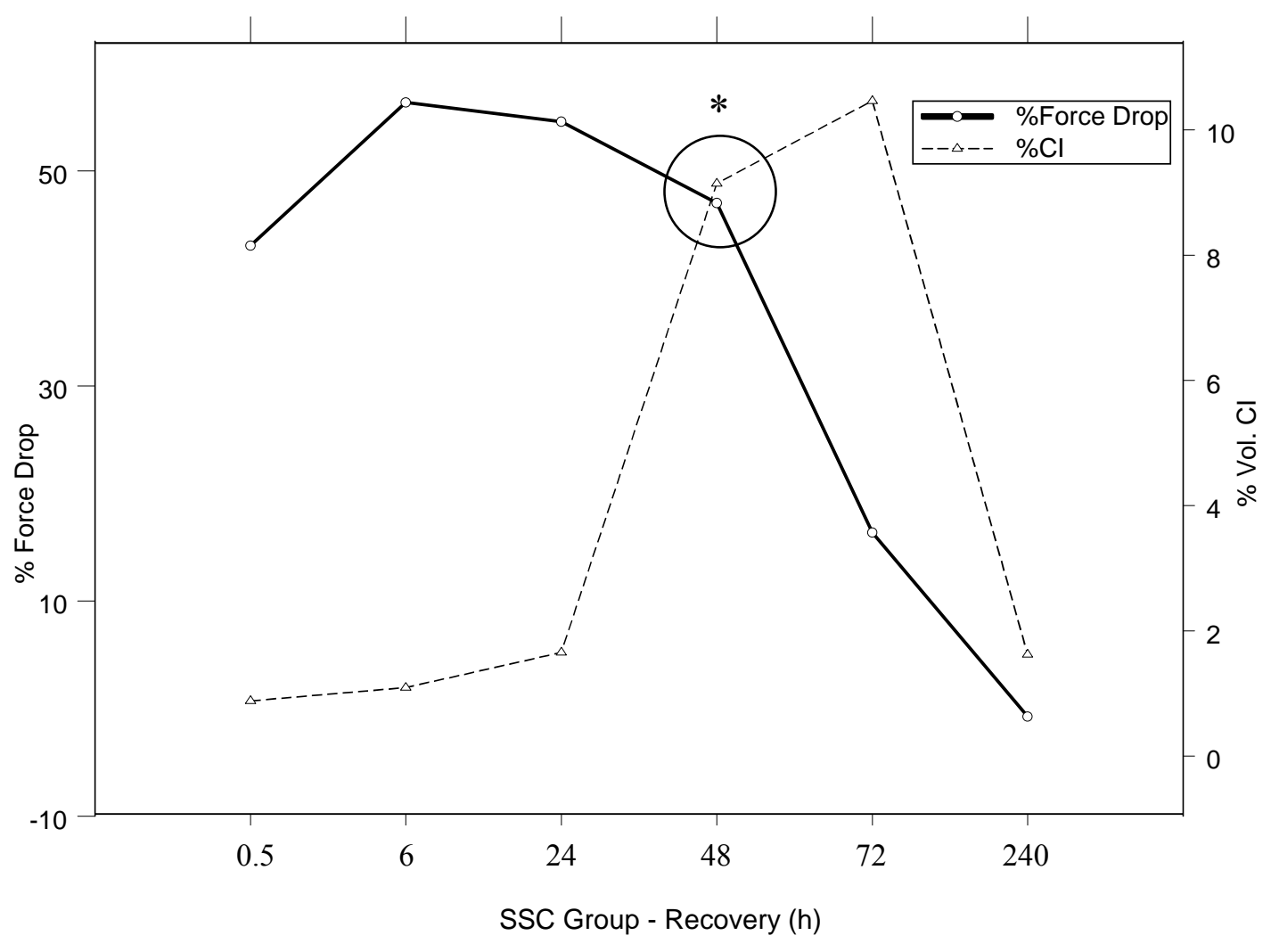


Figure 8c

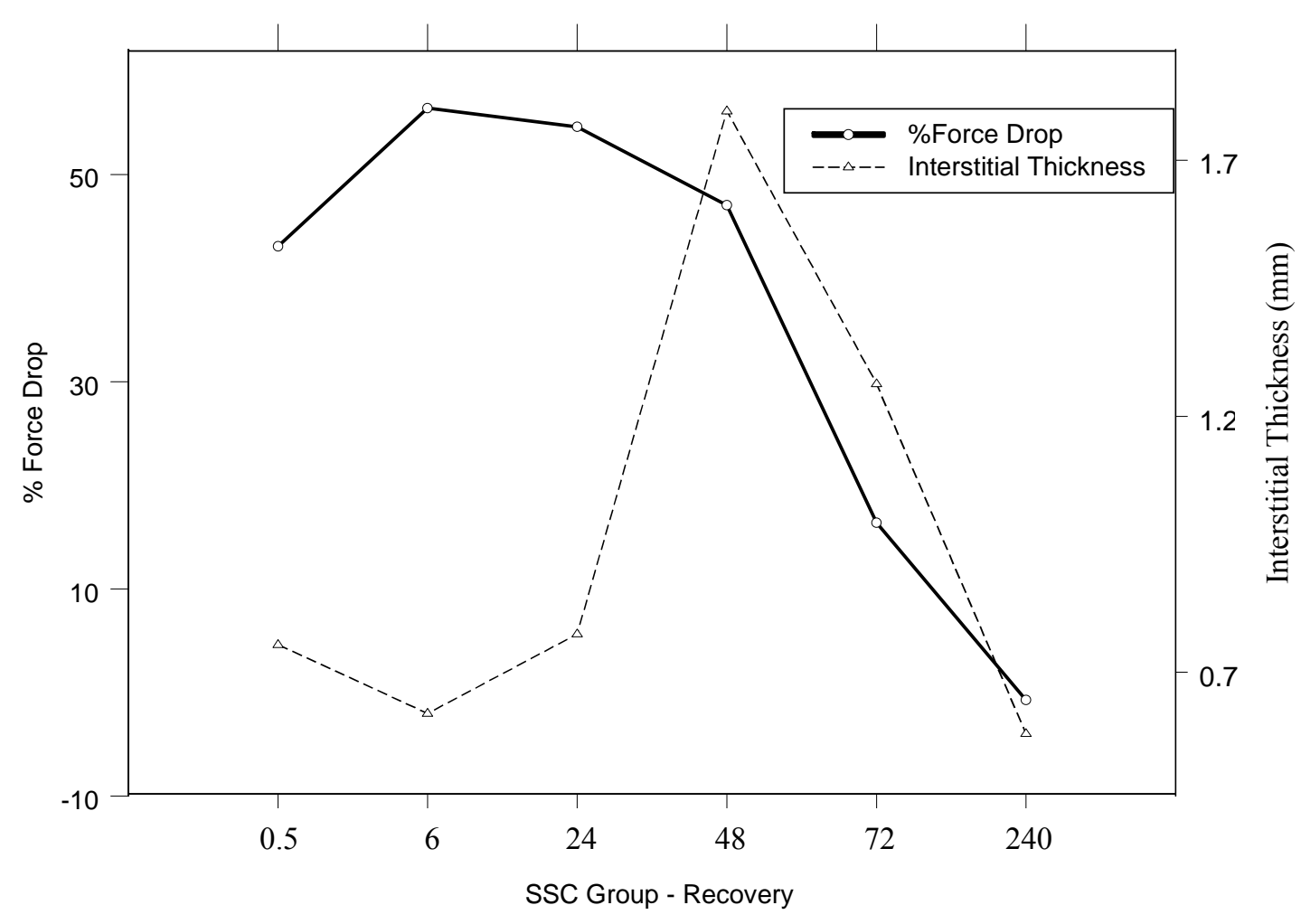


Impact of Repetition Number on Muscle Performance and Histological Response

\author{
B.A. Baker, MS ${ }^{1}$, R.R. Mercer, PhD $^{1}$, K.B. Geronilla, MS ${ }^{1}$, M.L. Kashon, \\ PhD $^{1}$, G.R. Miller, BS ${ }^{1}$, R.G. Cutlip, PhD $^{1}$ \\ ${ }^{1}$ National Institute for Occupational Safety and Health (NIOSH), Health Effects Laboratory \\ Division, Morgantown, WV, 26505, USA.
}

Disclaimer: The findings and conclusions in this report are those of the author(s) and do not necessarily represent the views of the National Institute for Occupational Safety and Health.

Address correspondence to:

Robert G. Cutlip, Ph.D.

1095 Willowdale Road MS L-2027

Morgantown, WV 26505

Phone: 304-285-5968

Fax: 304-285-6265

email: rgc8@cdc.gov

Running title: Stretch-Shorten Cycle Induced Muscle Injury 


\section{$\underline{\text { Abstract }}$}

Skeletal muscle injury is major concern in sport- and occupation-related fields. Purpose: We investigated the effects of increasing Stretch-shortening contraction (SSC) repetition number in vivo, and the resulting changes in functional performance and quantitative morphometry in rat skeletal muscle. Methods: Functional testing was performed on the ankle dorsiflexor muscles of Sprague-Dawley rats, which were randomly exposed to 30 SSCs, 70 SSCs, 150 SSCs, or 15 isometric contractions of equal duration. Following exposure, changes in functional performance and muscle morphometry were assessed at $48 \mathrm{~h}$ post-exposure. Stereology was used to quantify the volume density of degenerative myofibers and normal myofibers in the tibialis anterior muscle from each group as well as measures of inflammation and swelling, and changes in the interstitial space. Results: At $48 \mathrm{~h}$ there was a significant decline in isometric force for the 70 and $150 \mathrm{SSC}$ groups $(\mathrm{p}<0.05$ and $\mathrm{p}<0.05$, respectively). Stereological measures indicated a significant decrease in the percentage of volume density of normal myofibers in the 70 and $150 \mathrm{SSC}$ groups $(\mathrm{p}<0.05)$. Measures for percentage of volume density of degenerative myofibers and inflammation were increased ( $\mathrm{p}<0.0001$ and $\mathrm{p}<0.05$, respectively) in the 70 and 150 SSC groups. Moreover, a significant increase in the percentage of volume density of degenerative myofibers in the $150 \mathrm{SSC}$ group when compared to the $70 \mathrm{SSC}$ group was observed $(\mathrm{p}<0.05)$. Conclusion: These data strongly suggest that exposure to increasing $\mathrm{SSC}$ repetitions results in increased functional decrements and morphometric indices of myofiber degeneration and inflammation, and that there is an apparent threshold (repetition number) at which this occurs.

Keywords: Muscle Injury; Stereology; dorsiflexor muscles; in vivo 


\section{Introduction:}

Paragraph 1: Soft tissue injury (including skeletal muscle) is pronounced in the work-place, and accounts for $\sim 38 \%$ of all medically diagnosed injuries (Welch et al., 2005). This leads to a significant increase in lost workdays and increased disability (Welch, 1999). The use of muscle contractions to study skeletal muscle injury mechanics is beneficial in understanding the etiology of work-related musculoskeletal disorders. Findings from volitional animal models of repetitive motion (Barr and Barbe, 2004, Barbe and Barr, 2006), human models of exercise overload (Clarkson and Sayers, 1999, Carp et al., 2006, Ljung et al., 1999, Reid and MacGowan, 1998, Clarkson and Hubal, 2002), and electrically stimulated rat dynamometer models (Baker et al., 2006a, Baker et al., 2006b, Geronilla et al., 2003, Pizza et al., 2005) demonstrate that the cellular pathways of activation and the accompanying inflammation and histopathology are congruent.

Paragraph 2: Stretch-shortening contractions (SSCs - reciprocal eccentric/concentric contractions) are physiologic in nature and have been studied in the context of human locomotion and athletic performance (Avela and Komi, 1998). Exposure to SSCs have been shown to produce muscle injury due to the eccentric component of the cycle (Cutlip et al., 1997b, Cutlip et al., 2004, Geronilla et al., 2003, Horita et al., 1999). Thus, the amount of mechanical loading does have a graded effect on both changes in muscle performance and the extent of myofiber injury (Geronilla et al., 2003). In fact, varying the amount of repetitions may shape the physiological response in the continuum from adaptation to mal-adaptation and injury.

Paragraph 3: Studies of acute muscle injury have focused on the force response of muscle to a single exposure using a broad range of muscle contractions from 1-1800 repetitions (Brooks and 
Faulkner, 1990, Brooks et al., 1995, Lieber and Friden, 1988, Lieber et al., 1996). The results from these studies indicate that muscle injuries may be due solely to eccentric muscle actions. It is possible that while muscles are absorbing energy, mechanical damage may occur. High mechanical forces produced during eccentric muscle actions have been causal in the underlying etiology of muscle strain injuries (Warren et al., 1993a, Warren et al., 1993b). However, the influence of multiple repetitions on surpassing the target muscle's safety threshold, or tolerance level, and initiating an injury response is not fully understood.

Paragraph 4: There is clear evidence that the number of forced lengthening contractions has an effect on the amount of resultant muscle injury and force deficit (Hesselink et al., 1996). Hesselink and colleagues have demonstrated that in rat muscle $\sim 240$ stretches may be the threshold for inducing the maximum loss in isometric force, with an insignificant additional loss in isometric force encountered following 300 repetitions (Hesselink et al., 1996). Still, these results only allow us to conclude what may occur at the ceiling of contraction-induced muscle injury, while a very crucial component of contraction-induced muscle injury may be overlooked - the safety threshold. Single stretch models that have stretched muscle within the physiological range have not resulted in muscle damage or a pronounced force deficit (Brooks et al., 1995, Hunter and Faulkner, 1997). In other studies, it required more than one stretch within the physiological range to produce muscle injury (Geronilla et al., 2003, Warren et al., 1993a, Gosselin and Burton, 2002, Willems and Stauber, 2000, Willems and Stauber, 2001).

Paragraph 5: A variety of methods have been used to examine the physiological and cellular responses to contraction-induced muscle injury. Importantly, these methods have not established 
strict classification criteria for quantifying myofiber degeneration. Moreover, these methods did not address the interstitial space's response and modifications to the induced injury. By establishing rigorous exclusion criteria for categorizing cellular normality, degeneration, and inflammation, we recently have been able to rapidly and objectively determine the histological status of the muscle and interstitial space temporally following various SSC-injury exposures (Baker et al., 2006a, Baker et al., 2006b). However, the effect of repetition number in vivo using SSCs and the resulting quantitative degree of myofiber inflammation and degeneration has not been studied to date.

Paragraph 6: In the current study our purpose was to investigate the effects that increasing repetition number exhibited on rat skeletal muscle exposed in vivo to electrically evoked (supramaximal) SSCs of the left limb that activated the dorsiflexor muscle group, including the tibialis anterior muscle. The specific aims of the current study were twofold: (i) to investigate the effects that increasing repetition number has on skeletal muscle functional performance 48 hours following an acute SSC exposure and (ii) to investigate the effects that increasing repetition number has on skeletal muscle morphology 48 hours following an acute SSC exposure. Specifically, we hypothesized that animals exposed to increasing numbers of SSC repetitions will have increased functional performance decrements 48 hours following acute SSC exposure. Additionally, we hypothesized that animals exposed to increasing numbers of SSC repetitions will have increased stereological indices of myofiber injury, inflammation, and increased disruption to the interstitial space 48 hours following acute SSC exposure. 


\section{Methods}

Paragraph 7: Animals: Male Sprague-Dawley rats (422 \pm 19 g, 12 weeks of age) $(n=24)$ were used in the present study. Rats were housed in an AAALAC accredited animal quarters where the temperature and light/dark cycle (dark cycle from 7:00 AM to 7:00 PM) were controlled and food and water were provided ad libitum. All rats were exposed to a standardized experimental protocol approved by the NIOSH Animal Care and Use Committee that complied with the Guide for the Care and Use of Laboratory Animals. Animals were randomly assigned to either an isometric-control group (CON, $n=6), 30 \mathrm{SSC}$ group $(\mathrm{n}=6)$, a $70 \mathrm{SSC}$ group $(\mathrm{n}=6)$, or a 150 SSC group $(n=6)$. A sub-set of animals, which constituted the CON-group at $48 \mathrm{~h}$ and $150 \mathrm{SSC}$ group at 48-h recovery, were reported in a previous study (Baker et al., 2006a).

Paragraph 8: Experimental Setup: Animals were tested on a custom-built rodent dynamometer as previously described. Rat dorsi-flexor muscles were exposed to a SSC protocol as previously described by (Baker et al., 2006a). Briefly, rats were anesthetized with isoflurane gas in an "induction" tank (Surgivet Anesco Inc.), placed supine on a heated x-y positioning table of the rodent dynamometer, with an anesthetic mask over the nose and mouth of the animal. The knee was secured with a knee holder and the left foot (exposed limb) was secured in the load cell fixture with the ankle axis (assumed to be between the medial and lateral malleoli aligned with the axis of rotation of the load cell fixture). Each animal was monitored during the procedure to maintain proper anesthetic depth and body temperature.

Paragraph 9: Functional Testing: Platinum stimulating electrodes (catalogue \# F-E2, Grass Medical Instruments, Quincy MA, USA) were placed subcutaneously to span the common 
peroneal nerve. Activation of the electrical stimulator resulted in muscle contraction of the dorsi-flexor muscle group. Stimulator settings were optimized to maximize dorsi- flexor contractile performance as previously described (Geronilla et al., 2003). Muscle stimulation for all protocols was conducted at $120 \mathrm{Hertz}(\mathrm{Hz})$ stimulation frequency, 0.2-milliseconds (ms) pulse duration, and 4 Volts (V) in magnitude. The joint angle of the rat ankle was defined as the angle between the tibia and the plantar surface of the foot. The angular position of the load cell corresponded with the foot position. Vertical forces applied to an aluminum sleeve fitted over the dorsum of the foot were translated to a load cell transducer (Sensotec, Inc.) in the load cell fixture. The force produced by the dorsiflexor muscles was measured at the interface of the aluminum sleeve and the dorsum of the foot. An isometric contraction was performed at 1.57 radians (rad) ankle angle using $300 \mathrm{~ms}$ simulation duration. An isometric contraction was performed two minutes preceding (pre) and 2 minutes (min) following (post) either 3 sets of 10 SSCs (30 SSCs), 7 sets of 10 SSCs (70 SSCs), 15 sets of 10 SSCs (150 SSCs), or fifteen isometric contractions (CON group), and 48 hours post-SSC exposure (immediately prior to the animal being euthanized). All animals were returned to their home cages in the animal quarters after their respective treatment protocol and remained there with access to food and water ad libitum until time of sacrifice. Animals were monitored after their treatment exposure and did not show any signs of distress.

Paragraph 10: Injury Protocol: The contraction-induced injury groups were exposed to 30 SSCs, 70 SSCs, or 150 SSCs. The SSCs were electrically evoked (supra-maximal) via stimulation of the common peroneal nerve of the left limb, which activated the dorsiflexor muscle group, including the tibialis anterior muscle for $100 \mathrm{~ms}$, and then moving the load cell 
fixture from $1.13 \mathrm{rad}$ to $2.00 \mathrm{rad}$ angular position at a velocity of $8.72 \mathrm{rad} / \mathrm{s}$, in a reciprocal fashion, for 10 oscillations. After 10 oscillations, the load cell fixture was stopped at angular position $1.13 \mathrm{rad}$ and the dorsi-flexor group was deactivated $300 \mathrm{~ms}$ later. The total stimulation time per set was 2.8 seconds (s). The repetitions were conducted at 1-min intervals.

Paragraph 11: Isometric Control Protocol: The CON group was exposed to 15 isometric contractions at 1-min intervals. During each contraction, dorsi flexor muscles were stimulated for $2.8 \mathrm{~s}$ at $1.57 \mathrm{rad}$ using the same stimulation parameters and duration as in the SSC group.

Paragraph 12: Histology: Following completion of the $2^{\text {nd }}$ post-test rats from both the CON group and SSC group were weighed, anesthetized with sodium pentobarbital (ip, $10 \mathrm{mg} / 100 \mathrm{~g}$ body weight) and exsanguinated at $48 \mathrm{~h}$ following SSC exposure. The left tibialis anterior muscle (LTA) and right tibialis anterior muscle (RTA) were dissected, cleaned, and weighed. The mid-belly region was cut from the muscle and mounted on cork, immersed in optimal cutting temperature medium (OCT), frozen in isopentane cooled with liquid nitrogen, and stored at $-80^{\circ} \mathrm{C}$. The mid-belly region was selected to obtain the maximum tissue sample. Transverse sections were cut at 12 microns $(\mu \mathrm{m})$, mounted on pre-coated microscope slides, air dried, and stained using a routine procedure with Harris H\&E. Permount was used to attach coverslips to microscope slides. Stereological analyses were evaluated on a Leica DMLB microscope.

Paragraph 13: Myofiber Definitions: Stereology was used to quantify the degree of myofiber degeneration, and the accompanying changes in the interstitial space in the TA muscle from each group. Myofibers have been defined previously (Baker et al., 2006a), but briefly: Normal 
myofibers demonstrated: (i) Complete contact with adjacent myofibers, (ii) A smooth outer membrane, and (iii) No presence of internal inflammatory cells. Degenerative myofibers displayed: (i) A loss of contact with adjacent myofibers, (ii) Presence of internal inflammatory cells, and (iii) An outer membrane interdigitated with inflammatory cells.

Paragraph 14: Stereology: Quantitative morphometric methods were used to measure the volume fraction and surface densities of normal myofibers, degenerative myofibers, and the interstitial space as previously described (Underwood, 1970). The interstitium was divided into the endomysium and the perimysium space, which included capillaries. Stereology was also used to quantify the degree of inflammation, which was quantified as either non-cellular interstitium (NCI) indicative of edema, or cellular interstitium (CI); CI consisted of all possible infiltrating cells such as, but not limited to, inflammatory, endothelial, and fibroblasts. A stage micrometer was used to identify the mid-point of the sample section. Point and intercept counts using a 121point / 11-line overlay graticule (12.5 mm square with 100 divisions) at 40x magnification were taken at five equally spaced sites across the section. This process was repeated, $2 \mathrm{~mm}$ on either side of the mid-point of the section for a total of 1210- points and 110-intercept lines per section (Figure 1). Volume density or the percent (\%) tissue volume was computed from the percentage of points over the tissue section to points over normal myofibers, degenerative myofibers, CI, and NCI. Intercepts over the line overlay were counted for the perimeter of normal myofibers, degenerative myofibers, and interstitium to myofiber transitions. Points and intercepts over blood vessels greater than $25 \mu \mathrm{m}$ in diameter were excluded. 
Paragraph 15: Statistical Analysis: Statistical analyses were conducted using SAS Version 8 (SAS Institute, Cary NC). Mixed model analyses of variance with repeated measures were utilized for the analysis of isometric force measures. Post hoc analysis where main effects or interactions were significant was subsequently performed using Fisher's LSD. Stereological measurements of the volume of cellular and non-cellular components were analyzed using 2-way (treatment $\mathrm{x}$ limb) ANOVAs with the animal as the random factor accounting for measures in both limbs. Post-hoc comparisons were made using Fishers LSD tests. Data for the measurement of the percentage of volume density of degenerative myofibers was regarded as ordinal (due to the consensus of zero scores for the control and therefore the lack of variance that would be present as opposed to the exposed animals), so a nonparametric Kruskal-Wallis test was applied, and when differences were statistically significant, a Mann-Whitney U-test was performed. One section per animal with 6 animals per group was evaluated and the results expressed as mean \pm S.E.M.

\section{$\underline{\text { Results }}$}

Paragraph 16: Functional Measurements: No difference was observed in the maximum preexposure isometric force between any of the SSC groups or the isometric-control group $(\mathrm{p}=$ 0.4402, Figure 2). However, $48 \mathrm{hrs}$ following the exposure, the isometric control group exhibited a $5.6 \%$ increase in force, while animals exposed to the SSCs exhibited a loss of force of $14.9 \%$, $26.4 \%$, and $40.5 \%$ respectively for the $30 \mathrm{SSC}, 70 \mathrm{SSC}$, and $150 \mathrm{SSC}$ groups. Thus, at 48 hours post-exposure, the isometric force generated by the 150 SSC group was statistically lower than its pre-exposure isometric force test, the CON group $(\mathrm{p}<0.05)$, the $30 \mathrm{SSC}$ group $(\mathrm{p}<0.05)$, and 
the 70 SSC group ( $p<0.05$, Figure 2). However, all other comparisons were not statistically different.

\section{Quantitative Morphology:}

Paragraph 17: Stereological Analyses of Normal Myofibers: There was no difference in the percentage of volume density of normal myofibers in the contra-lateral control, isometriccontrol, or 30 SSC groups. However, there was a decrease in percentage of volume density of normal myofibers in the 70 and 150 SSC groups $(\mathrm{p}<0.05$, Figure 3$)$. Additionally, there was a significant decrease in the percentage of volume density of normal myofibers between the 70 and 150 SSC groups, with 150 SSCs resulting in a decreased percentage of volume density of normal myofibers $(\mathrm{p}<0.05$, Figure 3$)$.

Paragraph 18: Stereological Analyses of Degenerative Myofibers: SSC exposure did result in an increased percentage of volume density of degenerative myofibers in the 70 and 150 SSC groups ( $\mathrm{p}<0.0001$, Figure 4$)$. There was also a significant difference in the percentage of volume density of degenerative myofibers between the 70 and 150 SSC groups, with 150 SSCs resulting in a significantly greater percentage of volume density of degenerative myofibers ( $\mathrm{p}<$ 0.05, Figure 4). Representative images illustrate that none of the contra-lateral control muscles exhibited stereological indices for degenerative myofibers (Figure 5a). In addition, no degenerative myofibers were observed in the isometric-control muscles (Figure 5b). Surprisingly, there were no significant levels of degenerative myofibers in the 30 SSC group when compared to control groups (Figure 5c). As reported above, myofiber degeneration is 
present in histological images from both the 70 and $150 \mathrm{SSC}$ groups (Figures $5 \mathrm{~d}$ and $5 \mathrm{e}$, respectively).

Paragraph 19: Stereological Analyses of Inflammation: Non-cellular and cellular interstitium (indicative of edema and infiltration of inflammatory cells, respectively) was quantified to determine the overall inflammatory changes occurring following acute exposure. The percentage of volume density of non-cellular interstitium was not different in the contra-lateral control, isometric-control, or $30 \mathrm{SSC}$ groups (Figure 5a-c). Conversely, 70 and $150 \mathrm{SSCs}$ did result in an increase in the percentage of volume density of non-cellular interstitium when compared with the control groups $(\mathrm{p}<0.05$, Figure 4; Figure $5 \mathrm{~d}$ and $5 \mathrm{e})$. There was no difference in percentage of volume density of cellular interstitium in the control groups or $30 \mathrm{SSC}$ group, yet an increase in the percentage of volume of cellular interstitium was evident in the 70 and $150 \mathrm{SSC}$ groups when compared to control groups ( $\mathrm{p}<0.05$, Figure 4; Figure $5 \mathrm{~d}$ and $5 \mathrm{e})$.

\section{$\underline{\text { Discussion }}$}

Paragraph 20: The most significant finding of the current study was that increasing numbers of SSCs did produce increasing indices of myofiber degeneration and inflammation and, additionally, paralleled the decrease in functional deficit exhibited by the decline in isometric force production in the SSC groups. The morphological results were obtained using a systematic sampling technique (stereology) that is rapid and sensitive (a major advantage), and quite distinct from the approaches previously used to quantify muscle injury (Baker et al., 2006a, Baker et al., 2006b). Furthermore, a clear division was evident with respect to the number of SSC repetitions required to induce the subsequent inflammatory cascade and degenerative response, thus 
surpassing the TA's safety threshold at 70 SSC repetitions. In contrast, there was no myofiber degeneration or inflammatory response observed in the contra-lateral control group or the isometric-control group or $30 \mathrm{SSC}$ group. Additionally, the isometric control group displayed a significant recovery of isometric force $48 \mathrm{~h}$ post-exposure, whereas no such recovery was observed in the SSC-exposed groups. Our results are in agreement with previous results reported by Geronilla and colleagues (Geronilla et al., 2003), and further their initial observations that myofiber necrosis and myositis increased with increasing repetition number. Additionally, we observed an increasing quantity of degeneration and inflammation with increasing SSC number, and this increase clearly exhibits a dose-response finding (this is consistent and corroborates the findings of Hesselink and Colleagues (Hesselink et al., 1996).

Paragraph 21: As with previous reports (Hesselink et al., 1996), our findings are not consistent with those reported previously by others, most notably McCully and Faulkner (McCully and Faulkner, 1985, McCully and Faulkner, 1986). These authors reported that skeletal muscle damage "levels off" following a specified number of repetitions. Many likely explanations may be attributed to these data, which conflict with our present findings. First, the exposure protocols that were employed in the previous studies mentioned used forced lengthening contractions, whereas we used stretch-shortening cycle contractions to induce myofiber injury. In addition to the type of contraction involved, is the active or passive state of the contraction involved. A number of previous investigations have only used active lengthening contractions that are immediately followed by a passive shortening contraction. Although the aim of previous studies was to ascertain the involvement of lengthening contractions on skeletal muscle injury, this paradigm may not account for the physiological status of an active shortening phase contraction. 
Realizing the physiological relevance of this feature is important, for the results reported in previous works may overestimate the safety threshold for skeletal muscle in an in vivo preparation. Alternatively, the current protocol used may not have utilized enough repetitions to achieve the ceiling effect that has been reported. However our focus was not on attaining a ceiling effect, but to ascertain when the initiation of myofiber injury may first appear with respect to increasing repetition number. Secondly, as noted by Hesselink and colleagues (Hesselink et al., 1996) the target muscle investigated (tibialis anterior versus extensor digitorum longus) may contribute to variations in the response observed. Finally, the animal species used (e.g. mouse, rabbit, and rat) or even strain of rat used may possibly influence the observed results.

Paragraph 22: In summary, we observed an increase in the stereological indices for myofiber degeneration, non-cellular interstitium (edema), and cellular interstitium (cell infiltrates) with increasing SSC repetitions. These measures became significant and continued to increase at the 70 SSC repetition number, thus suggesting a clear threshold for the target muscle's safety threshold with increasing number of repetitions. Conversely, we did not see any measures increase in the isometric-control group or in the 30 SSC group. Moreover, functional capacity, assessed by isometric force production, was decreased in both the isometric-control group and the SSC groups. However, the decrease in force production was only observed at $48 \mathrm{~h}$ postexposure in the SSC groups; with the isometric-control group showing a full recovery. Thus, identifying the safety threshold of skeletal muscle with respect to increasing number of repetitions is of major importance when designing preventative strategies in vocational and recreation arenas, as well as for understanding the etiology of acute-loading injuries. These data 
strongly suggest that there is a level of exposure where the capacity to withstand the initial injury is compromised, and hence exceeds the muscle's safety threshold.

\section{Acknowledgments:}

The authors would like to thank Dr. Renguang Dong, Dr. Petia Simeonova, and Dr. Paul Nicolaysen of the National Institute for Occupational Safety and Health (NIOSH) for their critical review and comments regarding this manuscript. 


\section{References}

1. Avela, J. and P. V. Komi. Reduced stretch reflex sensitivity and muscle stiffness after long-lasting stretch-shortening cycle exercise in humans. Eur J Appl Physiol Occup Physiol. 78:403-410, 1998.

2. Baker, B. A., R. R. Mercer, K. B. Geronilla, M. L. Kashon, G. R. Miller, and R. G. Cutlip. Stereological analysis of muscle morphology following exposure to repetitive stretch-shortening cycles in a rat model. Appl Physiol Nutr Metab. 31:167-179, 2006.

3. Baker, B. A., K. M. Rao, R. R. Mercer, et al. Quantitative histology and MGF gene expression in rats following SSC exercise in vivo. Med Sci Sports Exerc. 38:463-471, 2006.

4. Barbe, M. F. and A. E. Barr. Inflammation and the pathophysiology of work-related musculoskeletal disorders. Brain Behav Immun. 20:423-429, 2006.

5. Barr, A. E. and M. F. Barbe. Inflammation reduces physiological tissue tolerance in the development of work-related musculoskeletal disorders. J Electromyogr Kinesiol. 14:77$85,2004$.

6. Brooks, S. V. and J. A. Faulkner. Contraction-induced injury: recovery of skeletal muscles in young and old mice. Am J Physiol. 258:C436-442, 1990.

7. Brooks, S. V., E. Zerba, and J. A. Faulkner. Injury to muscle fibres after single stretches of passive and maximally stimulated muscles in mice. J Physiol. 488 ( Pt 2):459-469, 1995.

8. Carp, S. J., M. F. Barbe, K. A. Winter, M. Amin, and A. E. Barr. Inflammatory biomarkers increase with severity of upper extremity overuse disorders. Clin Sci (Lond), 2006.

9. Clarkson, P. M. and M. J. Hubal. Exercise-induced muscle damage in humans. Am J Phys Med Rehabil. 81:S52-69, 2002.

10. Clarkson, P. M. and S. P. Sayers. Etiology of exercise-induced muscle damage. Can J Appl Physiol. 24:234-248, 1999.

11. Cutlip, R. G., K. B. Geronilla, B. A. Baker, M. L. Kashon, G. R. Miller, and A. W. Schopper. Impact of muscle length during stretch-shortening contractions on real-time and temporal muscle performance measures in rats in vivo. J Appl Physiol. 96:507-516, 2004. 
12. Cutlip, R. G., W. T. Stauber, R. H. Willison, T. A. McIntosh, and K. H. Means. Dynamometer for rat plantar flexor muscles in vivo. Med Biol Eng Comput. 35:540-543, 1997.

13. Geronilla, K. B., G. R. Miller, K. F. Mowrey, et al. Dynamic force responses of skeletal muscle during stretch-shortening cycles. Eur J Appl Physiol. 90:144-153, 2003.

14. Gosselin, L. E. and H. Burton. Impact of initial muscle length on force deficit following lengthening contractions in mammalian skeletal muscle. Muscle Nerve. 25:822-827, 2002.

15. Hesselink, M. K., H. Kuipers, P. Geurten, and H. Van Straaten. Structural muscle damage and muscle strength after incremental number of isometric and forced lengthening contractions. J Muscle Res Cell Motil. 17:335-341, 1996.

16. Horita, T., P. V. Komi, C. Nicol, and H. Kyrolainen. Effect of exhausting stretchshortening cycle exercise on the time course of mechanical behaviour in the drop jump: possible role of muscle damage. Eur J Appl Physiol Occup Physiol. 79:160-167, 1999.

17. Hunter, K. D. and J. A. Faulkner. Pliometric contraction-induced injury of mouse skeletal muscle: effect of initial length. J Appl Physiol. 82:278-283, 1997.

18. Lieber, R. L. and J. Friden. Selective damage of fast glycolytic muscle fibres with eccentric contraction of the rabbit tibialis anterior. Acta Physiol Scand. 133:587-588, 1988.

19. Lieber, R. L., L. E. Thornell, and J. Friden. Muscle cytoskeletal disruption occurs within the first 15 min of cyclic eccentric contraction. J Appl Physiol. 80:278-284, 1996.

20. Ljung, B. O., R. L. Lieber, and J. Friden. Wrist extensor muscle pathology in lateral epicondylitis. J Hand Surg [Br]. 24:177-183, 1999.

21. McCully, K. K. and J. A. Faulkner. Characteristics of lengthening contractions associated with injury to skeletal muscle fibers. J Appl Physiol. 61:293-299, 1986.

22. McCully, K. K. and J. A. Faulkner. Injury to skeletal muscle fibers of mice following lengthening contractions. J Appl Physiol. 59:119-126, 1985.

23. Pizza, F. X., J. M. Peterson, J. H. Baas, and T. J. Koh. Neutrophils contribute to muscle injury and impair its resolution after lengthening contractions in mice. $J$ Physiol.

562:899-913, 2005.

24. Reid, W. D. and N. A. MacGowan. Respiratory muscle injury in animal models and humans. Mol Cell Biochem. 179:63-80, 1998. 
25. Underwood, E. E. Quantitative Stereology. Reading, MA: Addison-Wesley Publishing Co, 1970, 23-34.

26. Warren, G. L., D. A. Hayes, D. A. Lowe, and R. B. Armstrong. Mechanical factors in the initiation of eccentric contraction-induced injury in rat soleus muscle. J Physiol. 464:457$475,1993$.

27. Warren, G. L., D. A. Hayes, D. A. Lowe, B. M. Prior, and R. B. Armstrong. Materials fatigue initiates eccentric contraction-induced injury in rat soleus muscle. J Physiol. 464:477-489, 1993.

28. Welch, L. S. Chronic symptoms in construction workers treated for musculoskeletal injuries. Am. J. Ind. Med. 36:532-540, 1999.

29. Welch, L. S., K. L. Hunting, and J. A. Murawski. Occupational injuries among construction workers treated in a major metropolitan emergency department in the United States. Scand J Work Environ Health. 31 Suppl 2:11-21, 2005.

30. Willems, M. E. and W. T. Stauber. Changes in force by repeated stretches of skeletal muscle in young and old female Sprague Dawley rats. Aging (Milano). 12:478-481, 2000.

31. Willems, M. E. and W. T. Stauber. Force deficits after repeated stretches of activated skeletal muscles in female and male rats. Acta Physiol Scand. 172:63-67, 2001. 


\section{$\underline{\text { Figure Captions }}$}

\section{Figure 1.}

Representative drawing of a tibialis anterior muscle cross-section prepared on a slide. The vertical, red mid-line represents the middle of the section, while the vertical black lines represent the sampling fields (separated from the mid-line by $2 \mathrm{~mm}$ on either side). Each horizontal arrow represents the equal-distant site that is sampled using a 121-point / 11-line overlay graticule.

\section{Figure 2.}

Isometric force change of muscles $48 \mathrm{~h}$ after SSCs of varying repetition numbers. Different letters denote significance $(A, B)$ and are reported at the $\mathrm{p}<0.05$ level. Group $(\mathrm{n}=6)$ data shown are mean values +/- S.E.M.

\section{Figure 3.}

The \% volume density of normal myofibers of muscles exposed to SSCs of varying repetition numbers. Different letters denote significance (A, B, C) and are reported at the $\mathrm{p}<0.05$ level. Group $(n=6)$ data shown are mean values +/- S.E.M.

\section{Figure 4.}

$\underline{\text { The } \% \text { volume density of degenerative myofibers, non-cellular interstitium, and cellular }}$ interstitium of muscles exposed to SSCs of varying repetition numbers. Different letters denote significance $(A, B, C)$ and are reported at the $p<0.05$ level. Group $(n=6)$ data shown are mean values +/- S.E.M. 


\section{Figure 5 (a-e):}

Hematoxylin and Eosin stained sections of the non-exposed tibialis anterior muscle (Figures 5aRTA (contra-lateral control)) and exposed tibialis anterior muscle (Figure $5 \mathrm{~b}-\mathrm{CON}$ (isometric control), Figure 5c-30 SSC group, Figure 5d-70 SSC group, Figure 5e- 150 SSC group), respectively. Micrographs shown are representative of the overall groups. The micrograph from the RTA, CON, and 30 SSC groups (Figure 5a, 5b, 5c) depict normal morphology. The micrograph from the $70 \mathrm{SSC}$ and $150 \mathrm{SSC}$ groups (Figure 5d, 5e) depict myofiber degeneration as well as cellular and non-cellular infiltrates. The 150 SSC group (Figure 5e) exhibits a significant increase in both degenerative myofibers and inflammation. All micrographs are captured at 40x magnification. 
Figure 1

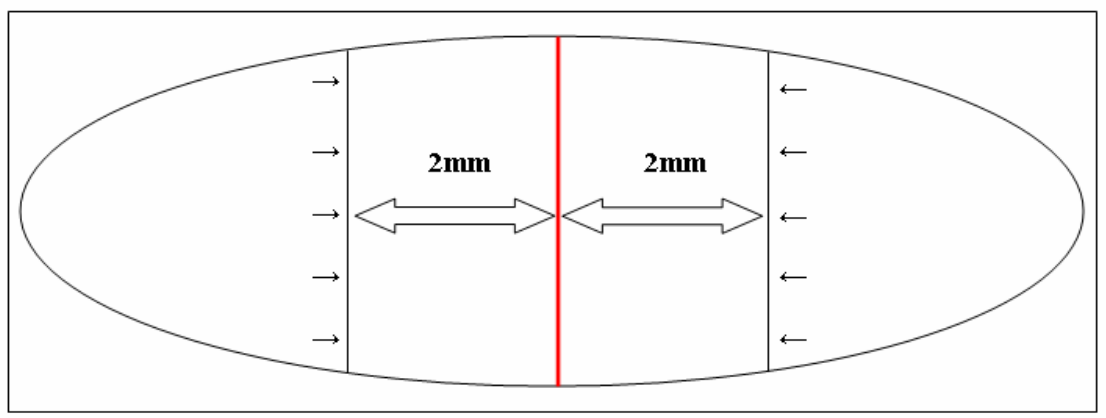


Figure 2

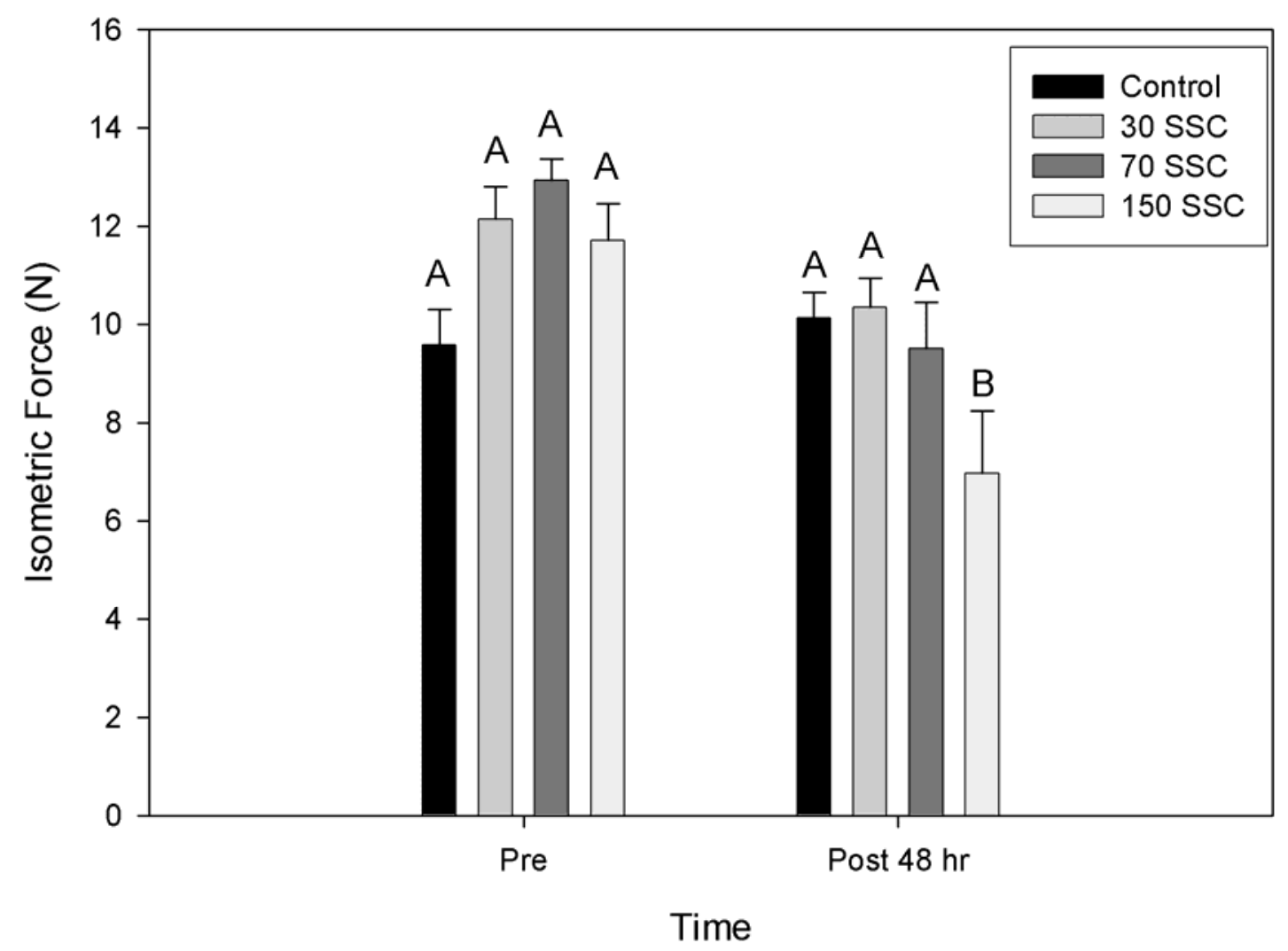


Figure 3

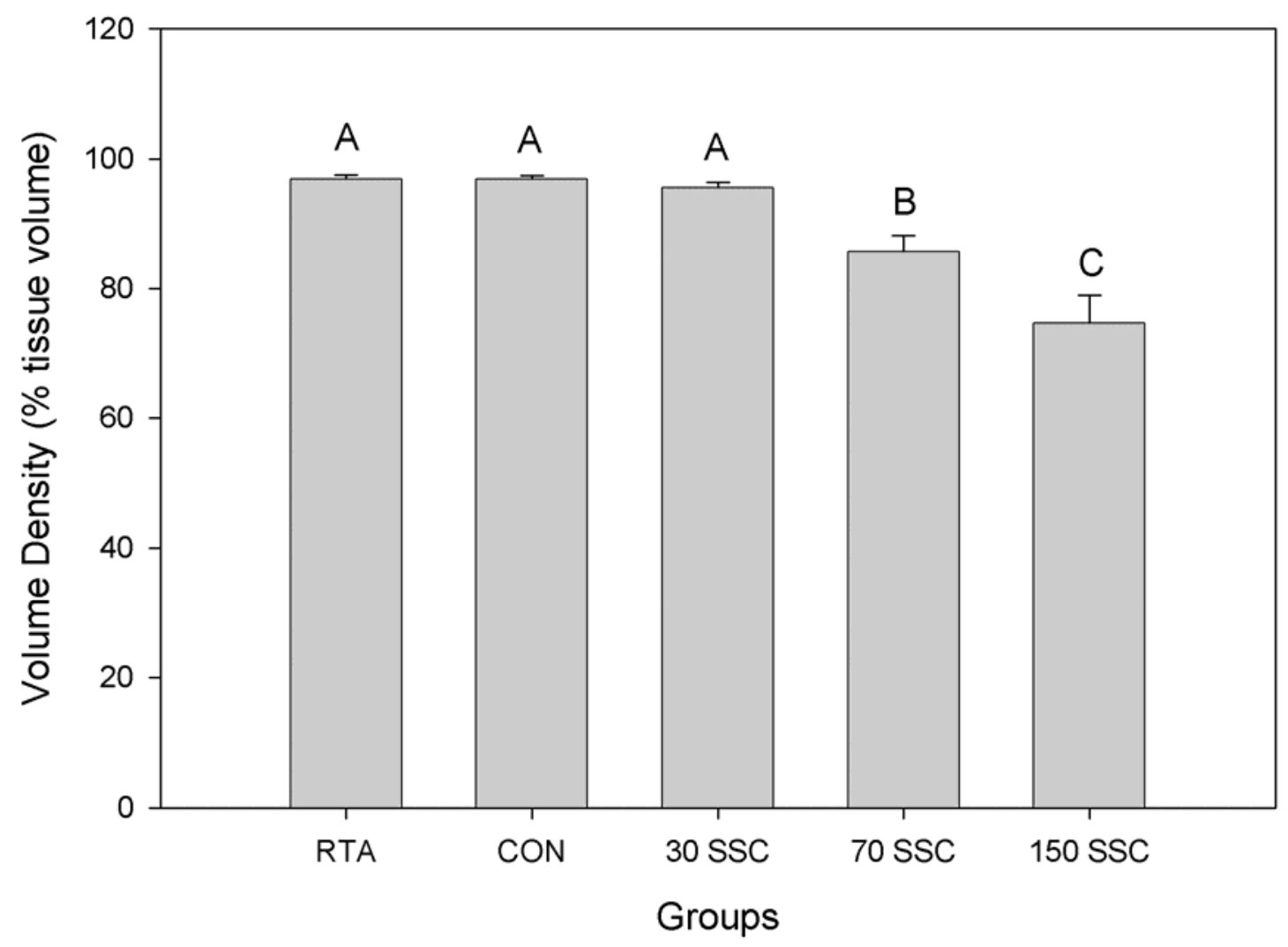


Figure 4

\section{Stereology}

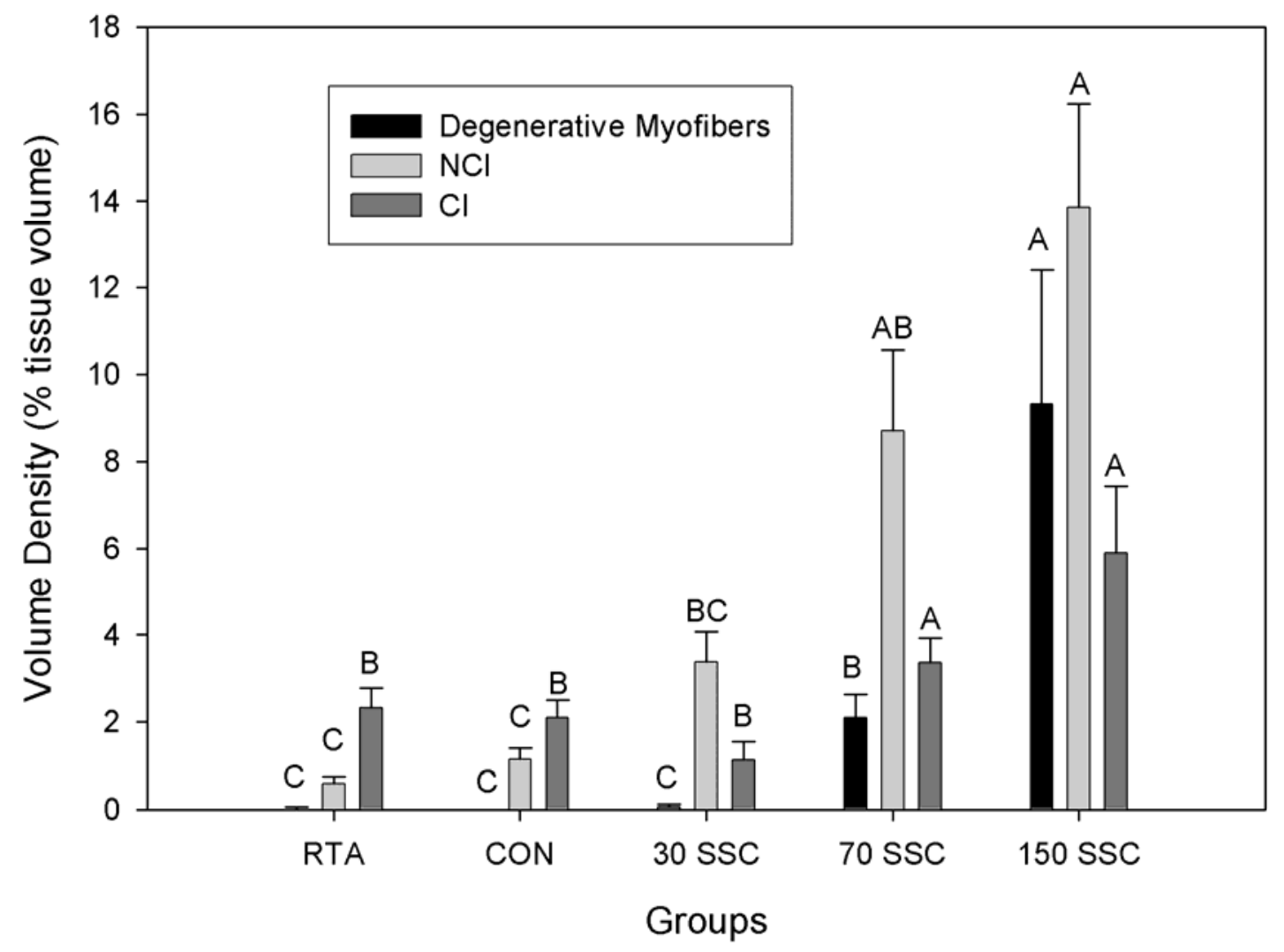


Figure 5
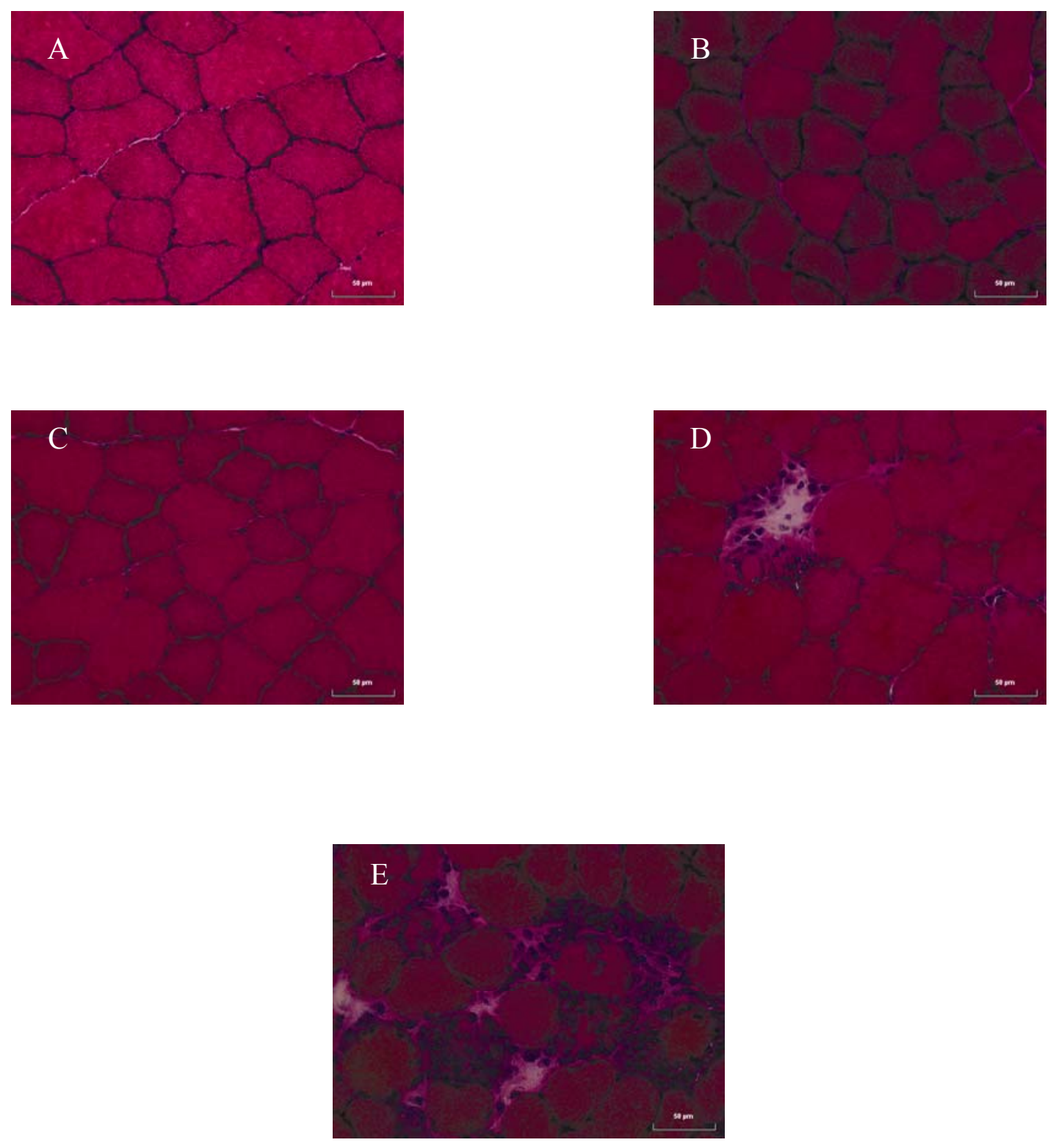
Adaptive Stretch-Shortening Contractions: Diminished Regenerative Capacity with Aging

\author{
B.A. Baker ${ }^{1}$, M.S. Hollander ${ }^{1}$, R.R. Mercer $^{1}$, M.L. Kashon ${ }^{1}$, and \\ R.G. Cutlip ${ }^{1}$

\footnotetext{
${ }^{1}$ National Institute for Occupational Safety and Health (NIOSH), Health Effects Laboratory Division, Morgantown, WV, 26505, USA.
}

Address correspondence to:

Robert G. Cutlip, Ph.D.

National Institute for Occupational Safety and Health

Health Effects Laboratory Division

1095 Don Nehlen Drive

$\mathrm{M} / \mathrm{S} 2027$

Morgantown, WV 26505

Fax: (304) 285-6265; Tel: (304) 285-5968; E-mail: rgc8@cdc.gov

Running head: Diminished Regenerative Capacity with Aging 


\begin{abstract}
The current study determined the acute events responsible for initiating adaptive processes and the influence age has on skeletal muscle regeneration. Changes in muscle performance and morphometry were quantified in young and old rodents following acute exposure to adaptive stretch-shortening contractions at $6,24,48,72$, and 120 hours post exposure $(n=6$ per age per recovery). Following the exposure old rodents were negatively influenced in all performance measures throughout the $120 \mathrm{~h}$ acute phase. Estimates of edema were increased in the old versus young exposed limb at $120 \mathrm{~h}$ recovery. Both young and old rodents displayed an increase in $\mathrm{MHC}_{\mathrm{dev}}{ }^{+}$labeling in the exposed limb, indicating muscle adaptation and regeneration. However, old rodents displayed diminished $\mathrm{MHC}_{\mathrm{dev}}{ }^{+}$labeling compared to young rodents, suggesting limited regenerative capacity. Based on these data, diminished muscle regeneration with aging may limit skeletal muscle following adaptive loading.
\end{abstract}

Keywords: Stretch-shortening contractions, dorsiflexor muscles, degenerative myofibers, inflammation, muscle regeneration

Disclaimer: "The findings and conclusions in this report are those of the author(s) and do not necessarily represent the views of the National Institute for Occupational Safety and Health." 


\section{INTRODUCTION}

Acute exposure to shortening (concentric) or isometric muscle actions does not normally produce muscle injury (Warren et al., 1993a, Warren et al., 1999b, Lieber et al., 1996). In contrast, exposure to lengthening (eccentric) movements and stretch-shortening contractions (SSCs reciprocal stretch-shortening contractions) has been shown to result in performance deficits (Geronilla et al., 2003, Warren et al., 1993b), myofiber degeneration (Baker et al., 2006a, Baker et al., 2006b), and inflammation (Baker et al., 2006a, Baker et al., In press-a). However, repeated mechanical exposures have been shown to result in adaptation, mal-adaptation, and even overt injury in both young and old age-groups (Brooks et al., 2001, McBride et al., 1995, Cutlip et al., 2006). While muscles from old animals are able to adapt to increased loads, aging inevitably diminishes the resulting muscle hypertrophy observed in old rodents (Degens and Alway, 2003, Alway et al., 2002), although the underlying mechanisms are poorly understood. After an injurious exposure to eccentric muscle contractions, there is an increased force deficit (Zerba and Faulkner, 1990) and slower recovery of performance (McBride et al., 1995) in whole muscles (McBride et al., 1995, Brooks and Faulkner, 1990, Brooks and Faulkner, 1996, Koh et al., 2003) and single fibers (Brooks and Faulkner, 1996) of old animals compared to young counterparts. Thus, identifying the acute events that are responsible for initiating the adaptive process and the differential control of these events with age in skeletal muscle is of major relevance, particularly when attempting to develop countermeasures in aging populations. Recently, we have investigated various input parameters owing to the induction of contractioninduced muscle injury (Baker et al., 2006a, Baker et al., 2006b, Baker et al., In press-a, Cutlip et al., 2004, Cutlip et al., 2005). Furthermore, from these studies, we have been able to optimize the exposure protocol (recovery kinetics, range of motion, duty cycle, number of repetitions, 
velocity) and produce substantial performance gains as well as muscle hypertrophy in young rodents versus old counterparts with as little as 4.5 weeks of training (Cutlip et al., 2006). The ability to prevent or protect against overt injury in older animals is thought to be attenuated due to higher eccentric force generation during eccentric muscle actions (Brooks and Faulkner, 1994) when compared to young animals, and this difference is magnified with increased stretch velocity (Eddinger et al., 1986). While previous data have suggested that muscle injury (myofiber degeneration) may be a prerequisite for adaptation, it is not known if this is absolutely required and, additionally, if chronic mal-adaptation results from an initial injurious exposure.

The purpose of this study was to quantify the acute-phase myofiber response in young and old rodents exposed to an acute bout of SSCs, which were optimized to elicit performance gains and muscle hypertrophy in young rodents subjected to chronic SSCs (Cutlip et al., 2006). Since previous literature has indicated that decreased performance and muscle injury is customary following lengthening-type movements and that aging exacerbates this response, we sought to determine whether aging affects the ability of skeletal muscle to respond functionally and morphologically to an acute, adaptive bout of SSCs. Identifying the underlying processes that impact the muscle's initial adaptive response with aging may be beneficial in optimizing current strategies for recovery and rehabilitation following loading of skeletal muscle.

\section{METHODS}

Animals: Male Fischer Brown Norway Hybrid rats (F344 x BN F1, N =60) were obtained from the National Institutes on Aging colony. Young adult $(\mathrm{N}=30,325 \mathrm{~g} \pm 29 \mathrm{~g} \mathrm{SD}, 12$ weeks of age) and old ( $\mathrm{N}=30,584 \mathrm{~g} \pm 43 \mathrm{~g} \mathrm{SD}, 30$ months) rats were housed in an AAALAC accredited animal quarters. Temperature and light/dark cycle (dark cycle from 7:00 AM to 7:00 PM) were held 
constant for all animals; food and water were provided ad libitum. After one week of acclimatization, all animals were segregated by age and randomized to SSC groups with 6, 24, 48,72 , or 120 hours $(h)$ recoveries $(n=6$ per recovery time). Each group was exposed to a standardized experimental protocol approved by the NIOSH Animal Care and Use Committee and euthanized at designated recovery points.

Experimental setup: The dorsiflexor muscles were tested on a custom-built rodent dynamometer (Cutlip et al., 1997a), as described previously (Cutlip et al., 2004). Briefly, the dynamometer provides precise control over the muscle length and muscle force output parameters to be studied. A Labview-based virtual instrument was developed that governed a National Instruments data acquisition board (PCI-MIO-16XE-10) and Unidex 100 motion controller (Aerotech Inc, Pittsburgh, PA, USA) for precise control of a brushless DC servomotor (1410 DC, Aerotech Inc, Pittsburgh, PA, USA) and muscle stimulator (Model SD9, Grass Medical Instruments, Quincy MA, USA). The software also acquired and stored position, force, and velocity data in real-time as described below.

Rats were anesthetized with $2 \%$ isoflurane gas using a small animal anesthetic system (Surgivet Anesco Inc., Waukesha, WI, USA). Isoflurane was chosen because it has no effect on in vivo force production (Ingalls et al., 1996). After anesthesia, each rat was placed supine on the heated $x-y$ positioning table of the rodent dynamometer, with an anesthetic mask placed over its nose and mouth. The knee was secured in flexion (at $90^{\circ}$ ) with a knee holder. The left foot was secured in the load cell fixture using a custom-built foot holder with the ankle axis (assumed to be between the medial and lateral malleoli) aligned with the axis of rotation of the load cell 
fixture. Each animal was monitored during the protocol to ensure proper anesthetic depth and body temperature.

Functional testing: As with the experimental set-up, the functional testing has been described in detail (Cutlip et al., 2004). Briefly though, the joint position of the animal was defined by the angle between the tibia and the plantar surface of the foot. The angular position of the load cell fixture corresponded with angular position of the ankle. The force produced by the dorsiflexor muscles was measured at the interface of the aluminum sleeve and the dorsum of the foot. Platinum stimulating electrodes (Grass Medical Instruments, Quincy MA, USA) were placed subcutaneously each exposure session to span the peroneal nerve. Activation of the electrical stimulator resulted in muscle contraction of the dorsiflexor muscle group.

Isometric and Dynamic Force Tests: Testing for isometric and single SSCs have been detailed in length previously (Cutlip et al., 2004). Briefly, an isometric and a single SSC were measured on the dorsi-flexor muscle group preceding and immediately following the acute SSC protocol as well as at each designated recovery point.

$\underline{\text { SSC protocol: }}$ The young and old age groups were exposed to 8 sets of 10 repetitions of SSCs. Within each set, there was a 2 second (s) rest between each SSC. For each repetition, the dorsiflexor muscles were fully activated for $100 \mathrm{~ms}$ duration via the electrical stimulator, and the eccentric contraction phase was initiated with a $60 \%$ s movement velocity of the load cell fixture over the prescribed range of motion of $90-140^{\circ}$ ankle angle. The load cell fixture was then immediately returned in the concentric phase at $60^{\circ} / \mathrm{s}$ to the starting position of $90^{\circ}$ ankle angle. 
The dorsiflexor muscles were then deactivated $300 \mathrm{~ms}$ later. Total stimulation time per repetition was $2.06 \mathrm{~s}$. The exposure paradigm was based on a recent version of the protocol that, when administered chronically, resulted in increased adaptation both functionally and morphologically in young rodents (Cutlip et al., 2006).

Muscle Processing: At the previously described recoveries following the exposure, rats were weighed, anesthetized with sodium pentobarbital (ip, $10 \mathrm{mg} / 100 \mathrm{~g}$ body weight) and exsanguinated. The left (exposed - LTA) and right (contra-lateral control - RTA) tibialis anterior muscles were dissected, weighed, and divided into 5 equal-distant, transverse "zones" ("Zone 1" most proximal - "Zone 5" most distal), and mounted onto cork board with OTC (VWR, West Chester, Pennsylvania), frozen in liquid nitrogen-cooled isopentane, and stored at $-80^{\circ} \mathrm{C}$ until sectioned for histology.

Histology and Immunohistochemistry: "Zone 3" was selected to obtain the maximum tissue sample; this corresponds to the TA mid-belly. Sequential, frozen transverse sections were cut at 12 micron $(\mu \mathrm{m})$ thickness, mounted on pre-coated microscope slides, air dried, and stored at $-80^{\circ}$ until processing. Once slide from the exposed and control limb of each rat was stained with hematoxylin \& eosin using Harris' procedure. Muscle morphology was assessed using quantitative morphometry (Underwood, 1970). Immunohistochemistry for developmental myosin heavy chain $\left(\mathrm{MHC}_{\mathrm{dev}}\right)$, a marker for muscle regeneration, was performed on one set of sections (one slide) from the exposed and control TA of each rodent. The frozen sections used for $\mathrm{MHC}_{\mathrm{dev}}$ immunolabeling were brought to room temperature and rinsed in PBS $+0.1 \mathrm{M}$ Glycine for 5 min. All slides were incubated in blocking buffer (5\% normal donkey serum 
diluted in PBS + Triton X-100) at RT for $2 \mathrm{~h}$. Slides were then incubated in either mouse anti$\mathrm{MHC}_{\mathrm{dev}}$ antibody diluted in blocking buffer at $1: 20$ for $1 \mathrm{~h}$ at $4^{\circ} \mathrm{C}$. Following incubation, sections were rinsed in PBS and incubated for $1 \mathrm{~h}$ at RT in Cy2 labeled donkey anti-mouse $\operatorname{IgG}(1: 200)$. Slides were then rinsed, incubated in DAPI to stain nuclei (1:1000, Sigma, St. Louis, MO), coverslipped using Prolong Gold antifade reagent (Molecular Probes), and allowed to dry in a cool, dark area. A number of slides were processed in the absence of the primary antibody as controls for non-specific binding, and tissue from $5 \mathrm{~d}$ rat pup and $5 \mathrm{~d}$ injured TA muscle were stained and served as positive controls.

Myofiber Definitions for Cross-sectional Stereology: Stereology was used to quantify the degree of myofiber degeneration, and the accompanying changes in the interstitial space in the TA muscle from each group. Myofibers have been defined previously (Baker et al., 2006a), but briefly: Normal myofibers demonstrated: (i) Complete contact with adjacent myofibers, (ii) A smooth outer membrane, and (iii) No presence of internal inflammatory cells. Degenerative myofibers displayed: (i) A loss of contact with adjacent myofibers, (ii) Presence of internal inflammatory cells, and (iii) An outer membrane interdigitated with inflammatory cells.

Stereology: Quantitative morphometric methods were used to measure the volume fraction, surface densities, and average thickness of normal myofibers, degenerative myofibers, and the interstitial space (Baker et al., 2006a). The interstitium was divided into endomysium and perimysium spaces, which included capillaries. A standardized stereological technique, as previously discussed (Baker et al., 2006a), was used to quantify the degree of myofiber degeneration and inflammation, which was quantified as either non-cellular interstitium (NCI), 
indicative of edema, or cellular interstitium (CI), indicative of cellular infiltrates. Fiber volume and surface density were measured using standard morphometric analyses (Underwood, 1970, Weibel, 1972, Weibel, 1974, Weibel, 1975). Briefly, one of the H\&E stained sections was taken from each animal. A stage micrometer was used to identify the mid-point of the section. Point and intercept counts using a 121-point / 11-line overlay graticule (12.5 mm square with 100 divisions) at 40x magnification were taken at 5 equally spaced points across the section. This process was repeated $2 \mathrm{~mm}$ on either side of the mid-point of the section for a total of 1210 points and 110 intercept lines per section. Volume density or percent volume was computed from the percentage of points over the tissue section to points over normal myofibers, degenerative myofibers, cellular interstitium and non-cellular interstitium plus capillaries (Weibel, 1972, Weibel, 1974, Weibel, 1975). Intercepts over the line overlay were counted for the perimeter of normal myofibers, degenerative myofibers, and interstitium to myofiber transitions. Points and intercepts over blood vessels greater than 25 microns $(\mu \mathrm{m})$ in diameter were excluded. Average thickness or average distance was computed from two times the ratio of volume to surface density according to standard morphometric analysis (Underwood, 1970). One section per animal with an $\mathrm{n}=6$ for young and old animals per group was evaluated and the results expressed as mean \pm S.E.M.

$\underline{\text { MHC }}_{\text {dev }}$ Localization and Quantification: Photomicrographs were captured and saved using an Olympus Photomicroscope and Simple PCI Image Analysis Software. Quantification of positively immunolabeled $\mathrm{MHC}_{\mathrm{dev}}$ sections was performed using 1 section per slide from the contra-lateral control RTA and exposed LTA muscles from each group. Digital color images were analyzed using standard quantitative morphometric methods (Weibel, 1972, Weibel, 1974, 
Weibel, 1975, Underwood, 1970) to measure the volume density (\% tissue fraction) of $\mathrm{MHC}_{\mathrm{dev}}^{+}$ positively labeled myofibers. Briefly, each of the MHC sections was quantified independently, as one section per slide was analyzed from each animal per group and quantified using standard morphometric techniques (Underwood, 1970). Volume density was computed from the percentage of points over the tissue section to points over each of the $\mathrm{MHC}_{\mathrm{dev}}{ }^{+}$labeled myofibers. MHC data are reported as mean $\pm \mathrm{SE}$ and analyzed as described below.

Muscle quality: Pre-test isometric force measured at the last session for each respective recovery was normalized to muscle wet-weight of the tibialis anterior of the exposed limb obtained at sacrifice as previously described (Degens and Alway, 2003, Cutlip et al., 2006).

\section{Data Analysis:}

Performance data: Isometric forces for respective recovery points were compared using a twoway ANOVA (age x time). Single SSC force and work data were compared similarly. All data are presented as means \pm SEM unless otherwise noted, and differences with a probability of $\mathrm{p}<$ 0.05 were considered significant.

Body weights, muscle wet-weights, and muscle quality: Data for body weight differences between the young and old rodents were analyzed using a two-sample t-test procedure assuming unequal variances. Data for muscle wet-weight differences were analyzed using a three-way (age x limb x time) ANOVA. Muscle quality measurements were analyzed using a two-way (age $\mathrm{x}$ time) ANOVA. All data are presented as means \pm SEM unless otherwise noted, and differences with a probability of $\mathrm{p}<0.05$ were considered significant. 
$\underline{\text { Stereology and MHC }} \underline{\text { dev }}$ IHC: Stereological measurements for volume and thickness of cellular and non-cellular components and muscle quality were analyzed using three-way ANOVA (age $\mathrm{x}$ limb $\mathrm{x}$ time) with the animal as the random factor accounting for measures in both limbs. $\mathrm{MHC}_{\mathrm{dev}}$ volume density was analyzed using two-way mixed model (age x limb) ANOVA with the animal as the random factor accounting for measures in both limbs. All data are presented as means \pm SEM unless otherwise noted, and differences with a probability of $\mathrm{p}<0.05$ were considered significant. All data were analyzed using JMP v. 5.1 (SAS Institute Inc., Cary, NC).

\section{$\underline{\text { RESULTS }}$}

\section{Functional Performance}

Isometric performance: Post-test isometric force was decreased in young and old rodents $6 \mathrm{~h}$ following exposure, irrespective of age ( $p<0.05$; Figure 1). Age negatively influenced the ability to produce isometric force, as old rodents' post-test isometric force values were decreased compared with young rodents' post-test isometric force values from $24-120 \mathrm{~h}(\mathrm{p}<0.05$; Figure $1)$.

\section{Single SSC (Dynamic performance):}

Peak eccentric force, minimum force, and negative and positive work were all influenced similarly, as post-test measures were decreased in young and old rodents at $6 \mathrm{~h}$ following exposure, irrespective of age $(\mathrm{p}<0.05$; Figures $2 \mathrm{a}$ and $2 \mathrm{~b}, 3 \mathrm{a}$ and $3 \mathrm{~b})$. Further, age negatively affected the ability of old rodents to produce dynamic force and work compared with young rodents from $24-120 \mathrm{~h}(\mathrm{p}<0.05$; Figures $2 \mathrm{a}$ and $2 \mathrm{~b}, 3 \mathrm{a}$ and $3 \mathrm{~b})$. 


\section{$\underline{\text { Biological Measures }}$}

Muscle wet-weights: Body weights and tibialis anterior muscle wet-weights of the exposed limb and contra-lateral control limb were normalized to tibia length following the acute exposure (Table 1). There was a significant interaction between age and time for muscle wet-weight $(\mathrm{p}<$ 0.05). LTA muscle wet-weight was increased compared to the RTA muscle wet-weight in the young rodents at 6 h recovery $(\mathrm{p}<0.05$, Figure 4$)$, and the LTA muscle wet-weight was increased compared to the RTA muscle wet-weight in old rodents at $24 \mathrm{~h}$ recovery $(\mathrm{p}<0.05$, Figure 4) as a result of exposure. Further analyses revealed young rodent's LTA muscle wetweight was significantly increased compared with older counterparts at $6 \mathrm{~h}, 72 \mathrm{~h}$, and $120 \mathrm{~h}(\mathrm{p}<$ 0.05; Figure 4).

Muscle quality: Age significantly impacted the mean value of isometric force normalized to muscle wet-mass $(\mathrm{p}<0.001)$, as muscle quality was $0.015 \pm 0.001 \mathrm{~N} / \mathrm{mg}$ and $0.001 \pm 0.0004$ $\mathrm{N} / \mathrm{mg}$ for the young and old groups, respectively, (Fig. 5a). The young group had a significantly greater muscle quality in the exposed limb at $24 \mathrm{~h}, 48 \mathrm{~h}$, and $120 \mathrm{~h}$ when compared with $6 \mathrm{~h}$ recovery $(\mathrm{p}<0.001$, Figure $5 b)$.

Stereological analyses of normal and degenerative myofibers: Figure 6 depicts representative micrographs of tibialis anterior cross sections from the exposed limb of young and old rodents at 24,72 , and 120 recoveries. No changes in normal myofibers were observed between any of the groups (Figure 7a). Statistical analysis showed that there was degenerative myofibers present only in TA muscle analyzed from the young rodents at $72 \mathrm{~h}$ recovery $(\mathrm{p}=0.049$, Figure $7 \mathrm{~b})$, and 
that this response was $<1 \%$ of the total tissue fraction. No significant degenerative myofibers were present in TA muscles of old rodents.

Stereological analyses of inflammation: NCI was significantly increased at $120 \mathrm{~h}$ recovery in the LTA of old rodents when compared to the LTA of young rodents $(\mathrm{p}<0.05$, Figure 5 (arrowheads) and Figure 7c). At $6 \mathrm{~h}, 24 \mathrm{~h}$, and $48 \mathrm{~h}$ recovery NCI was increased in the old rodent's contra-lateral limb when compared with young counterparts $(\mathrm{p}<0.05$; Figure $7 \mathrm{c})$. Conversely, the volume density of CI was significantly increased in the exposed muscles, irrespective of age at $72 \mathrm{~h}$ recovery, but this value returned to baseline by $120 \mathrm{~h}$ of the acute phase $(p<0.05$, Figure $7 d)$.

IHC for Developmental Myosin Heavy Chain: Representative images of $\mathrm{MHC}_{\mathrm{dev}}{ }^{+}$myofibers at $120 \mathrm{~h}$ (Figure 8a). At $120 \mathrm{~h}$, the volume of $\mathrm{MHC}_{\mathrm{dev}}{ }^{+}$muscle cells were increased in young rodents compared with old rodents, regardless of limb (Figure 8b). Exposure did increase the volume of $\mathrm{MHC}_{\mathrm{dev}}{ }^{+}$muscle cells versus the contra-lateral control limb, irrespective of age (Figure 8b). Further, the volume of $\mathrm{MHC}_{\mathrm{dev}}{ }^{+}$positive muscle cells was increased in the exposed limb of young rodents compared with their old counterparts. $(p<0.05$, Figure $8 b)$

\section{DISCUSSION}

The major findings of this study are that dorsiflexor muscles from young and old rodents exposed to an acute bout of 80 SSCs do not undergo the extent of myofiber degeneration $(<1 \%$ degenerative myofibers present) that is typically reported for classical contraction-induced muscle injury (Baker et al., 2006a, Baker et al., In press-a, Hesselink et al., 1996, McCully and 
Faulkner, 1985). Additionally, developmental myosin was expressed but to a much lesser extent in the old rodents compared with young rodents. This suggests that the signal to respond to the mechanical stimulus is intact in old rodents, but they were unable fully to meet the demands of the exposure. Moreover, in this study, developmental myosin was expressed in rodent tissue that did not exhibit signs of overt skeletal muscle injury. However, this diminished signal in myofibers that are clearly undergoing adaptation may suggest why old rodents do not have the capacity to adapt to repetitive exposures when compared with their young counterparts. This may also help explain why old rodents were negatively affected functionally compared to young rodents. Additionally, in the absence of muscle degeneration (necrosis), the inflammatory response (both cellular and non-cellular components) may contribute significantly to the collective functional and biological changes observed.

Acute exposures to eccentric-type exercise result in functional decrements that have a well-documented temporal response independent of age (Gosselin, 2000, Koh et al., 2003, Zerba and Faulkner, 1990), while aging exacerbates this response (Zerba et al., 1990, Cutlip et al., 2006, Krajnak et al., 2006). Our results indicate that an acute bout of adaptive SSCs do not follow the same pattern of functional decrement in young rodents as others have reported, since following the current exposure protocol, isometric force and dynamic performance recover as early as $24 \mathrm{~h}$ after the initial exposure. Yet, aging negatively influences functional performance as revealed by our old rodents' inability to recover throughout the $120 \mathrm{~h}$ acute phase. Due to the contraction protocol implemented, it would be anticipated that the lengthening component of the SSCs used would be adequate to induce significant myofiber degeneration (Baker et al., 2006a, Smith et al., 1997, Baker et al., In press-a, Geronilla et al., 2003). This is supported by numerous investigations where exposure to lengthening contractions (or contractions including a 
lengthening component) has resulted in long-lasting isometric force deficit (Warren et al., 1999b, Baker et al., 2006a), structural disruption at the cellular level, and cellular infiltrates as a result of the ensuing inflammatory response (Warren et al., 1999b, Lieber et al., 1996, Faulkner et al., 1989, Warren et al., 1993c, McCully and Faulkner, 1985, Friden et al., 1983b, Baker et al., 2006a, Baker et al., In press-a). Furthermore, the "initial" mechanical injury would be worsened and followed by an inflammatory cascade that is well-documented and known to result in a heightened "secondary" muscle injury (Armstrong, 1990, Tidball, 1995, Toumi, 2006). Recently, we have demonstrated that TA muscles from old rodents compared with young counterparts exhibited long-lasting functional decrements following a single injurious SSC exposure, and this was associated with increased estimates of edema as well as increased satellite cell apoptosis (Krajnak et al., 2006).

In the current study, there was a low level of myofiber degeneration present following $24 \mathrm{~h}$ recovery and in the contra-lateral limb in both the young and old rodents. As suggested by others (Smith et al., 1997) this strongly indicates that there is a low percentage of myofibers undergoing regular turnover (degeneration/ regeneration). However, this normal turnover is insignificant and does not contribute to skeletal muscle injury, while acute exposure to various modes of mechanical stimuli (i.e. physical activity/exercise) has been shown to increase this response and aging further exacerbates this effect (Baker et al., 2006a, Brooks and Faulkner, 1996, Smith et al., 1997). This is also substantiated by the observation that a small degree of myofibers labeled positively for developmental myosin. Understanding the degenerative/ regenerative process in skeletal muscle and whether or not degeneration is a prerequisite for myofiber regeneration is fundamental in our understanding of adaptation following mechanical exposures. Even though aged muscle does have a delayed recovery from a single injurious 
exposure, there appears to be the capacity for adaptation (Conboy et al., 2005), and the current study corroborates this proposition; as old rodents did display increased $\mathrm{MHC}_{\mathrm{dev}}{ }^{+}$labeling in the exposed TA muscle compared with the non-exposed limb. However, this response was diminished significantly compared to exposed TA muscles from young rodents in the current study. Collectively, the paucity of studies investigating the differential influences that result from single versus multiple exposures to the same type of activity, and the contribution that each impacts the resulting functional and biological plasticity limits our understanding of muscle adaptation under these variable conditions. Nevertheless, the current acute protocol suggests that even in the absence of overt skeletal muscle injury, aging severely limits the regenerative capacity of skeletal muscle following mechanical exposure, and, further, when this mechanical exposure protocol is administer chronically the resultant exposure eventually leads to significant mal-adaptation (Cutlip et al., 2006).

Stereological indices for non-cellular interstitium (edema) were increased with aging in the exposed limb by $35 \%$ at $120 \mathrm{~h}$. Thus, unresolved inflammatory changes may have contributed to the decreased functional performance at $120 \mathrm{~h}$. Whether an acute increase in NCI (edema) provides an environment that aggravates long-term signaling and recruitment of inflammatory cells is not known. Here we have demonstrated that the CI of the exposed limb in the aged rodents had returned to control values by $120 \mathrm{~h}$, while NCI continued to remain elevated at this time. In a chronic exposure using this protocol we observed an increase only in CI, with no accompanying increases in edema (Cutlip et al., 2006). Under both conditions we have illustrated that there was no significant myofiber degeneration present in either young or old rodents following this specific SSC exposure protocol. Thus, the alterations in NCI present at $120 \mathrm{~h}$ in our old rodents may contribute to long-term mal-adaptation by negatively modifying 
their local internal environment and ultimately affect muscle regeneration. This is plausible because alterations in the host environment with aging suggest that systemic factors may adversely affecting local tissue regeneration (Conboy et al., 2005).

Since the primary purpose of this study was to quantify the acute-phase functional and morphological response in young and old rodents following an acute bout of adaptive SSCs, the novelty of the data and findings should direct future investigation in improving the early regenerative signals in aged skeletal muscle. Also, identifying the acute inflammatory events (in the absence of myofiber degeneration) that may be responsible for initiating the earliest signaling events in skeletal muscle's adaptive process is essential, particularly when attempting to develop countermeasures in aging populations. Nonetheless, aging negatively affects functional performance (both static and dynamic) as well as changes in cellular permeability (indicated by an unresolved edematous response) that may impair the ability of skeletal muscles from older animals to adapt to an acute mechanical exposure. Evidently, old rodents are unable to overcome this initial mechanical insult due to the diminished expression of $\mathrm{MHC}_{\mathrm{dev}}{ }^{+}$in myofibers of the exposed TA muscle. 


\section{$\underline{\text { References }}$}

ALWAY, S. E., DEGENS, H., KRISHNAMURTHY, G. \& SMITH, C. A. (2002) Potential role for Id myogenic repressors in apoptosis and attenuation of hypertrophy in muscles of aged rats. Am J Physiol Cell Physiol, 283, C66-76.

ARMSTRONG, R. B. (1990) Initial events in exercise-induced muscular injury. Med Sci Sports Exerc, 22, 429-35.

BAKER, B. A., MERCER, R. R., GERONILLA, K. B., KASHON, M. L., MILLER, G. R. \& CUTLIP, R. G. (2006a) Stereological analysis of muscle morphology following exposure to repetitive stretch-shortening cycles in a rat model. Appl Physiol Nutr Metab, 31, 16779.

BAKER, B. A., MERCER, R. R., GERONILLA, K. B., KASHON, M. L., MILLER, G. R. \& CUTLIP, R. G. (In press) Impact of SSC Repetition Number on Muscle Performance and Histological Response. Med Sci Sports Exerc.

BAKER, B. A., RAO, K. M., MERCER, R. R., GERONILLA, K. B., KASHON, M. L., MILLER, G. R. \& CUTLIP, R. G. (2006b) Quantitative histology and MGF gene expression in rats following SSC exercise in vivo. Med Sci Sports Exerc, 38, 463-71.

BROOKS, S. V. \& FAULKNER, J. A. (1990) Contraction-induced injury: recovery of skeletal muscles in young and old mice. Am J Physiol, 258, C436-42.

BROOKS, S. V. \& FAULKNER, J. A. (1994) Isometric, shortening, and lengthening contractions of muscle fiber segments from adult and old mice. Am J Physiol, 267, C50713.

BROOKS, S. V. \& FAULKNER, J. A. (1996) The magnitude of the initial injury induced by stretches of maximally activated muscle fibres of mice and rats increases in old age. $J$ Physiol, 497 ( Pt 2), 573-80.

BROOKS, S. V., OPITECK, J. A. \& FAULKNER, J. A. (2001) Conditioning of skeletal muscles in adult and old mice for protection from contraction-induced injury. J Gerontol A Biol Sci Med Sci, 56, B163-71.

CONBOY, I. M., CONBOY, M. J., WAGERS, A. J., GIRMA, E. R., WEISSMAN, I. L. \& RANDO, T. A. (2005) Rejuvenation of aged progenitor cells by exposure to a young systemic environment. Nature, 433, 760-4.

CUTLIP, R. G., BAKER, B. A., GERONILLA, K. B., MERCER, R. R., KASHON, M. L., MILLER, G. R., MURLASITS, Z. S. \& ALWAY, S. E. (2006) Chronic exposure of stretch-shortening contractions results in skeletal muscle adaptation in young rats and maladaptation in old rats. Applied Physiology, Nutrition and Metabolism, 31, 573-587.

CUTLIP, R. G., GERONILLA, K. B., BAKER, B. A., CHETLIN, R. D., HOVER, I., KASHON, M. L. \& WU, J. Z. (2005) Impact of stretch-shortening cycle rest interval on in vivo muscle performance. Med Sci Sports Exerc, 37, 1345-55.

CUTLIP, R. G., GERONILLA, K. B., BAKER, B. A., KASHON, M. L., MILLER, G. R. \& SCHOPPER, A. W. (2004) Impact of muscle length during stretch-shortening contractions on real-time and temporal muscle performance measures in rats in vivo. $J$ Appl Physiol, 96, 507-16.

CUTLIP, R. G., STAUBER, W. T., WILLISON, R. H., MCINTOSH, T. A. \& MEANS, K. H. (1997) Dynamometer for rat plantar flexor muscles in vivo. Medical \& Biological Engineering \& Computing, 35, 540-3. 
DEGENS, H. \& ALWAY, S. E. (2003) Skeletal muscle function and hypertrophy are diminished in old age. Muscle Nerve, 27, 339-47.

EDDINGER, T. J., CASSENS, R. G. \& MOSS, R. L. (1986) Mechanical and histochemical characterization of skeletal muscles from senescent rats. Am J Physiol, 251, C421-30.

FAULKNER, J. A., JONES, D. A. \& ROUND, J. M. (1989) Injury to skeletal muscles of mice by forced lengthening during contractions. Q J Exp Physiol, 74, 661-70.

FRIDEN, J., SJOSTROM, M. \& EKBLOM, B. (1983) Myofibrillar damage following intense eccentric exercise in man. Int J Sports Med, 4, 170-6.

GERONILLA, K. B., MILLER, G. R., MOWREY, K. F., WU, J. Z., KASHON, M. L., BRUMBAUGH, K., REYNOLDS, J., HUBBS, A. \& CUTLIP, R. G. (2003) Dynamic force responses of skeletal muscle during stretch-shortening cycles. Eur J Appl Physiol, 90, 144-53.

GOSSELIN, L. E. (2000) Attenuation of force deficit after lengthening contractions in soleus muscle from trained rats. J Appl Physiol, 88, 1254-8.

HESSELINK, M. K., KUIPERS, H., GEURTEN, P. \& VAN STRAATEN, H. (1996) Structural muscle damage and muscle strength after incremental number of isometric and forced lengthening contractions. J Muscle Res Cell Motil, 17, 335-41.

INGALLS, C. P., WARREN, G. L., LOWE, D. A., BOORSTEIN, D. B. \& ARMSTRONG, R. B. (1996) Differential effects of anesthetics on in vivo skeletal muscle contractile function in the mouse. Journal of Applied Physiology, 80, 332-40.

KOH, T. J., PETERSON, J. M., PIZZA, F. X. \& BROOKS, S. V. (2003) Passive stretches protect skeletal muscle of adult and old mice from lengthening contraction-induced injury. J Gerontol A Biol Sci Med Sci, 58, 592-7.

KRAJNAK, K., WAUGH, S., MILLER, R., BAKER, B., GERONILLA, K., ALWAY, S. E. \& CUTLIP, R. G. (2006) Proapoptotic factor bax is increased in satellite cells in the tibialis anterior muscles of old rats. Muscle Nerve, 24, 720-730.

LIEBER, R. L., THORNELL, L. E. \& FRIDEN, J. (1996) Muscle cytoskeletal disruption occurs within the first 15 min of cyclic eccentric contraction. $J$ Appl Physiol, 80, 278-84.

MCBRIDE, T. A., GORIN, F. A. \& CARLSEN, R. C. (1995) Prolonged recovery and reduced adaptation in aged rat muscle following eccentric exercise. Mech Ageing Dev, 83, 185$\underline{200 .}$

MCCULLY, K. K. \& FAULKNER, J. A. (1985) Injury to skeletal muscle fibers of mice following lengthening contractions. J Appl Physiol, 59, 119-26.

SMITH, H. K., PLYLEY, M. J., RODGERS, C. D. \& MCKEE, N. H. (1997) Skeletal muscle damage in the rat hindlimb following single or repeated daily bouts of downhill exercise. Int J Sports Med, 18, 94-100.

TIDBALL, J. G. (1995) Inflammatory cell response to acute muscle injury. Med Sci Sports Exerc, 27, 1022-32.

TOUMI, H., F'GUER, S., BEST, T.M. (2006) The role of neutrophils in injury and repair following muscle stretch. J. Anat., 208, 459-470.

UNDERWOOD, E. E. (1970) Quantatative Stereology, Reading, MA, Addison-Wesley Publishing Co.

WARREN, G. L., HAYES, D. A., LOWE, D. A. \& ARMSTRONG, R. B. (1993a) Mechanical factors in the initiation of eccentric contraction-induced injury in rat soleus muscle. $J$ Physiol, 464, 457-75. 
WARREN, G. L., HAYES, D. A., LOWE, D. A., PRIOR, B. M. \& ARMSTRONG, R. B. (1993b) Materials fatigue initiates eccentric contraction-induced injury in rat soleus muscle. J Physiol, 464, 477-89.

WARREN, G. L., LOWE, D. A. \& ARMSTRONG, R. B. (1999) Measurement tools used in the study of eccentric contraction-induced injury. Sports Medicine, 27, 43-59.

WARREN, G. L., LOWE, D. A., HAYES, D. A., KARWOSKI, C. J., PRIOR, B. M. \& ARMSTRONG, R. B. (1993c) Excitation failure in eccentric contraction-induced injury of mouse soleus muscle. J Physiol, 468, 487-99.

WEIBEL, E. R. (1972) The value of stereology in analysing structure and function of cells and organs. J Microsc, 95, 3-13.

WEIBEL, E. R. (1974) Selection of the best method in stereology. J Microsc, 100, 261-9.

WEIBEL, E. R. (1975) Quantitation in morphology: possibilities and limits. Beitr Pathol, 155, 117.

ZERBA, E. \& FAULKNER, J. A. (1990) A Single Lengthening Contraction can Induce Injury to Skeletal Muscle Fibers. Physiologist, 33, A122.

ZERBA, E., KOMOROWSKI, T. E. \& FAULKNER, J. A. (1990) Free radical injury to skeletal muscles of young, adult, and old mice. Am J Physiol, 258, C429-35. 


\section{Table and Figure Legends}

Table 1: Body weight, absolute TA wet-weights, and normalized right and left TA muscle wet-

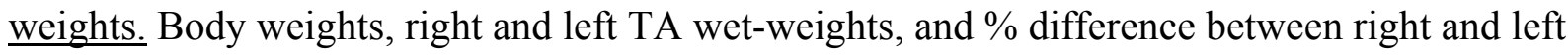
TA muscles are shown as mean values \pm standard error. Each row signifies body weight and the respective normalized muscle wet-weights at the specified recovery point. The left panel of the table represents the young rodents, while the right panel of the table represents the old rodents.

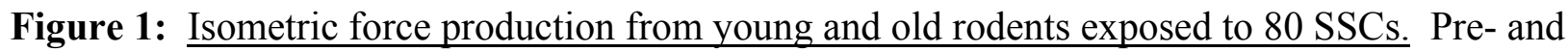
post-isometric force production in the exposed limb of young and old rodents, preceding exposure and throughout the $120 \mathrm{~h}$ acute phase. Old rodents were negatively influenced in their ability to produce isometric force following exposure to $80 \mathrm{SSCs}$. Significance is reported at the 0.05 level and is indicated by different letters. Data are reported as mean values \pm standard error.

Figure 2: Single SSC functional parameters obtained from the single SSC test from young and old rodents exposed to 80 SSCs. A) Peak eccentric force $\left(F_{\text {peak }}\right)$ and B) Minimum force $\left(F_{\min }\right)$ responded similarly, as older age negatively affected dynamic performance throughout the $120 \mathrm{~h}$ acute phase. Significance is reported at the 0.05 level and is indicated by different letters. Data are reported as mean values \pm standard error.

Figure 3: Single SSC work parameters obtained from the single SSC from young and old rodents exposed to $80 \mathrm{SSCs}$. A) Older age negatively affected the ability to absorb work ( $\mathrm{p}=$ 0.0005). B) Older age negatively affected the ability to generate work $(\mathrm{p}<0.0001)$. 
Significance is reported at the 0.05 level and is indicated by different letters. Data are reported as mean values \pm standard error.

Figure 4: Muscle wet-weights of the exposed (left) and control (right) TA muscle (standardized to tibia length) for young and old rodents following exposure to a single SSC protocol. LTA muscle wet-weight was increased compared to the RTA muscle wet-weight in the young rats at 6h recovery ( $\mathrm{p}<0.05$ ), and the LTA muscle wet-weight was increased compared to the RTA muscle wet-weight in old rats at $24 \mathrm{~h}$ recovery $(\mathrm{p}<0.05)$ as a result of exposure. Young rodent's LTA muscle wet-weight was increased compared with older rodents LTA at $6 \mathrm{~h}, 72 \mathrm{~h}$, and $120 \mathrm{~h}$ ( $p=0.002, p=0.005$, and $p<0.001$, respectively). Letters A (limb) and B (age) indicate significance and is reported at the 0.05 level. Data are reported as mean values \pm standard error.

Figure 5: Isometric force normalized to muscle wet weight $(\mathrm{N} / \mathrm{mg})$ of the tibialis anterior following the acute SSC exposure. A) Young rodents had greater muscle quality compared to older counterparts $(\mathrm{p}<0.001)$. B) Further, analysis for temporal muscle quality shows young rodents had significantly greater muscle quality at $24 \mathrm{~h}, 48 \mathrm{~h}$, and $120 \mathrm{~h}$ compared to $6 \mathrm{~h}$ ( $\mathrm{p}<$ 0.001). Letters indicate significance of age and are reported at the 0.05 level. Data are reported as mean values \pm standard error.

Figure 6: Morphological changes in muscle tissue from young and old rodents following exposure to SSCs. No myofiber or interstitial changes were observable in contra-lateral control tissue from young or old rodents. A) Micrograph representing the young exposed limb at $24 \mathrm{~h}$ recovery (note, no myofiber or interstitial disruption present). B) Representative micrograph 
from the young exposed limb at $72 \mathrm{~h}$ recovery displaying $<1 \%$ of myofibers having a degenerative response and also an increased cellular interstitial response (increased CI, arrows). C) Representative micrograph from the young exposed limb at $120 \mathrm{~h}$ recovery, which exhibited few fibers with central nuclei (arrows). D) Micrograph representing the old exposed limb at 24h recovery (note, as with young counterparts, no myofiber or interstitial disruption present). E) Representative micrograph from the old exposed limb at $72 \mathrm{~h}$ recovery displaying an increased cellular interstitial response (increased CI) as well as an increased swelling of the perimysium and endomysium (increased NCI, arrowheads). F) Representative micrograph from the old exposed limb at $120 \mathrm{~h}$ recovery, as observed with young rodents few fibers exhibited central nuclei (arrows). Scale bar shown is $50 \mu \mathrm{m}$. All micrographs are captured at 40X magnification.

Figure 7: The percent volume of normal and degenerative myofibers and cellular and noncellular interstitial components of inflammation from young and old rodents exposed (left) and control (right) TA muscles. Significance is reported at the 0.05 level. Data are reported as mean values \pm standard error.

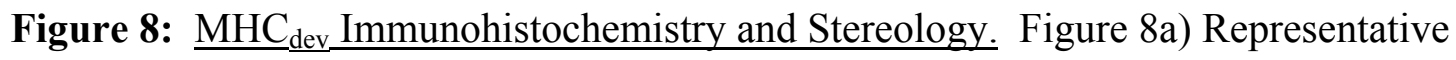
micrographs of MHCdev+ labeled muscle cells at 120h from exposed limbs of A) young and B) old rodents, as well as positive controls from C) SSC injured TA muscle and D) rat pup TA muscle. Scale bar shown is $50 \mu \mathrm{m}$. All micrographs were captured at 40X magnification. Figure $8 \mathrm{~b})$ At $120 \mathrm{~h}$ the $\%$ volume of $\mathrm{MHC}_{\mathrm{dev}}{ }^{+}$muscle cells was increased in young rodents compared with old rodents, regardless of limb. Exposure did increase the $\%$ volume of $\mathrm{MHC}_{\mathrm{dev}}{ }^{+}$muscle cells versus the contra-lateral control limb, irrespective of age. Further, the volume of $\mathrm{MHC}_{\mathrm{dev}}{ }^{+}$ 
muscle cells was increased in the exposed limb of young rodents compared with old counterparts $(p<0.05)$. Significance is reported at the 0.05 level and is indicated by different letters. Data are reported as mean values \pm standard error. 


\section{Table 1}

\begin{tabular}{|c|c|c|c|c|c|c|c|c|}
\hline \multicolumn{5}{|c|}{ Young Rodents } & \multicolumn{4}{|c|}{ Old Rodents } \\
\hline $\begin{array}{c}\text { Recovery } \\
\text { (hours) }\end{array}$ & $\begin{array}{l}\text { Body Weight } \\
\text { (grams) }\end{array}$ & $\begin{array}{c}\text { Normalized } \\
\text { RTA } \\
(\mathrm{mg} / \mathrm{mm})\end{array}$ & $\begin{array}{c}\text { Normalized } \\
\text { LTA } \\
(\mathrm{mg} / \mathrm{mm})\end{array}$ & $\begin{array}{c}\% \\
\text { Difference }\end{array}$ & $\begin{array}{l}\text { Body } \\
\text { Weight } \\
\text { (grams) }\end{array}$ & $\begin{array}{c}\text { Normalized } \\
\text { RTA } \\
(\mathrm{mg} / \mathrm{mm})\end{array}$ & $\begin{array}{c}\text { Normalized } \\
\text { LTA } \\
(\mathrm{mg} / \mathrm{mm})\end{array}$ & $\begin{array}{c}\% \\
\text { Difference }\end{array}$ \\
\hline $6 \mathrm{~h}$ & $322.7 \pm 10.1$ & $14.6 \pm 0.4$ & $16.4 \pm 0.4$ & $12.2 \pm 1.6$ & $592.3 \pm 18.0$ & $14.2 \pm .04$ & $15.0 \pm 0.2$ & $6.4 \pm 4.0$ \\
\hline $24 \mathrm{~h}$ & $296.2 \pm 7.3$ & $14.1 \pm 0.5$ & $15.0 \pm 0.5$ & $6.7 \pm 1.1$ & $560.2 \pm 12.4$ & $14.5 \pm 0.4$ & $16.0 \pm 0.3$ & $10.1 \pm 3.0$ \\
\hline $48 \mathrm{~h}$ & $319.5 \pm 7.0$ & $15.0 \pm 0.2$ & $15.7 \pm 0.2$ & $4.9 \pm 1.3$ & $561.6 \pm 20.3$ & $14.6 \pm 0.4$ & $15.0 \pm 0.4$ & $2.7 \pm 2.1$ \\
\hline $72 \mathrm{~h}$ & $347.3 \pm 8.9$ & $15.6 \pm 0.2$ & $16.6 \pm 0.3$ & $6.7 \pm 0.7$ & $591.2 \pm 28.2$ & $14.8 \pm 0.3$ & $15.1 \pm 0.5$ & $2.6 \pm 2.4$ \\
\hline $120 \mathrm{~h}$ & $344.1 \pm 12.8$ & $15.6 \pm 0.4$ & $15.6 \pm 0.5$ & $-0.4 \pm 1.8$ & $568.6 \pm 7.0$ & $13.6 \pm 0.6$ & $14.4 \pm 0.5$ & $5.9 \pm 1.8$ \\
\hline
\end{tabular}


Figure 1

Isometric Force

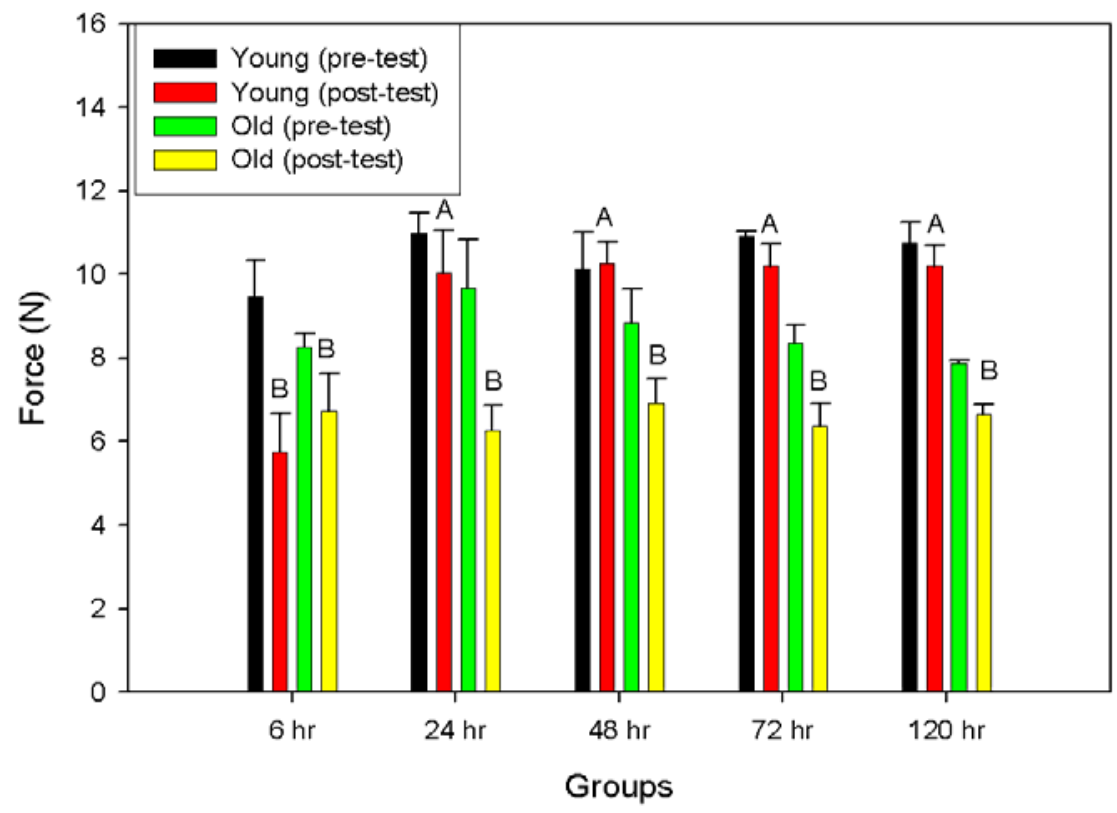


Figure 2a

Peak Eccentric Force (Fpeak)

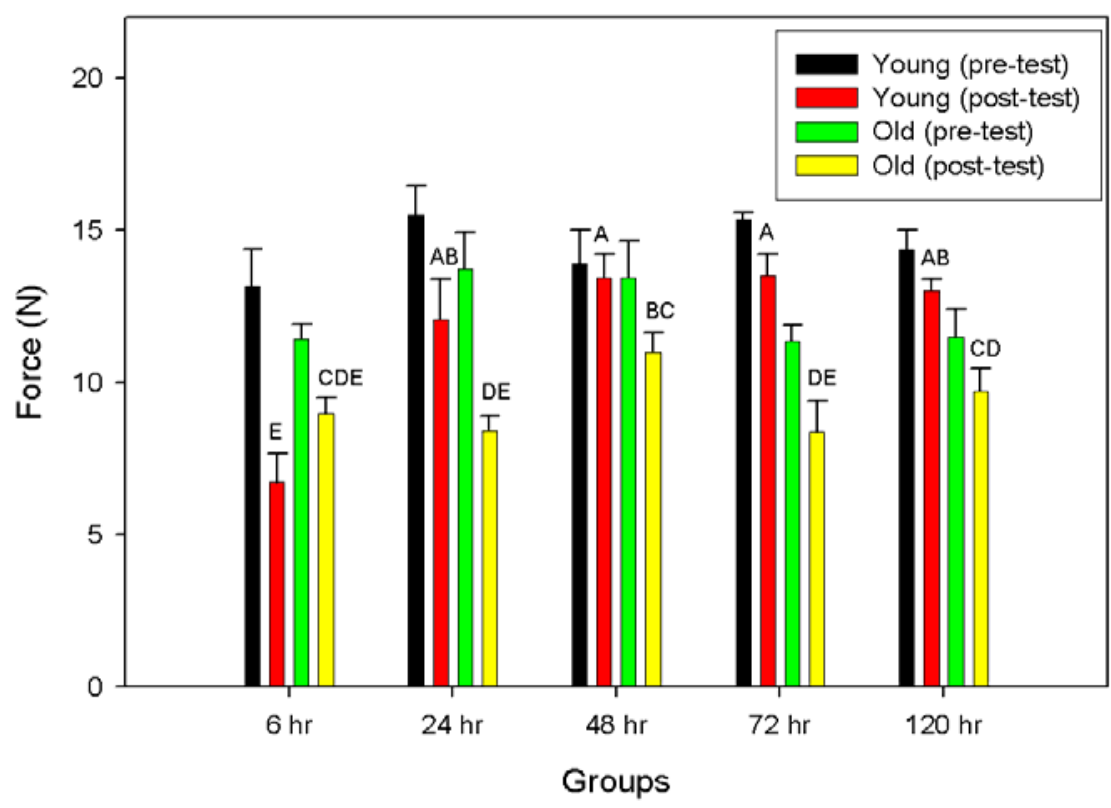

Figure 2b

Minimum Force (Fmin)

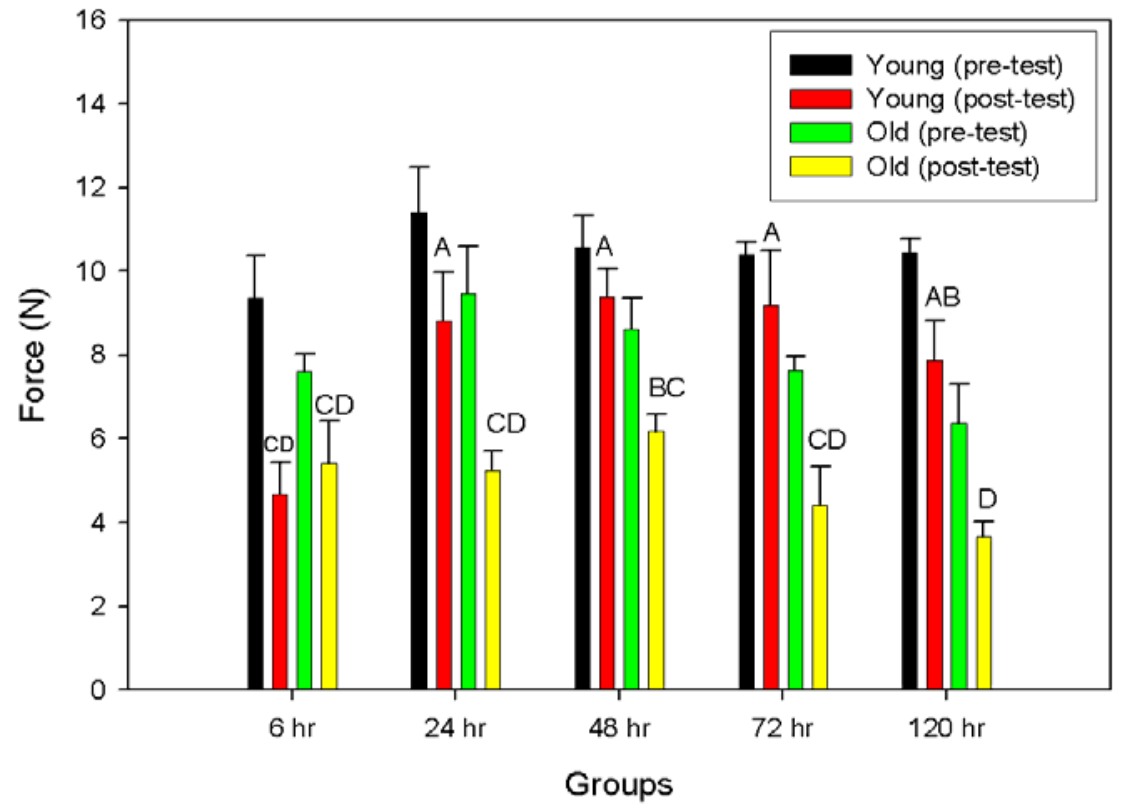


Figure 3a

Negative Work

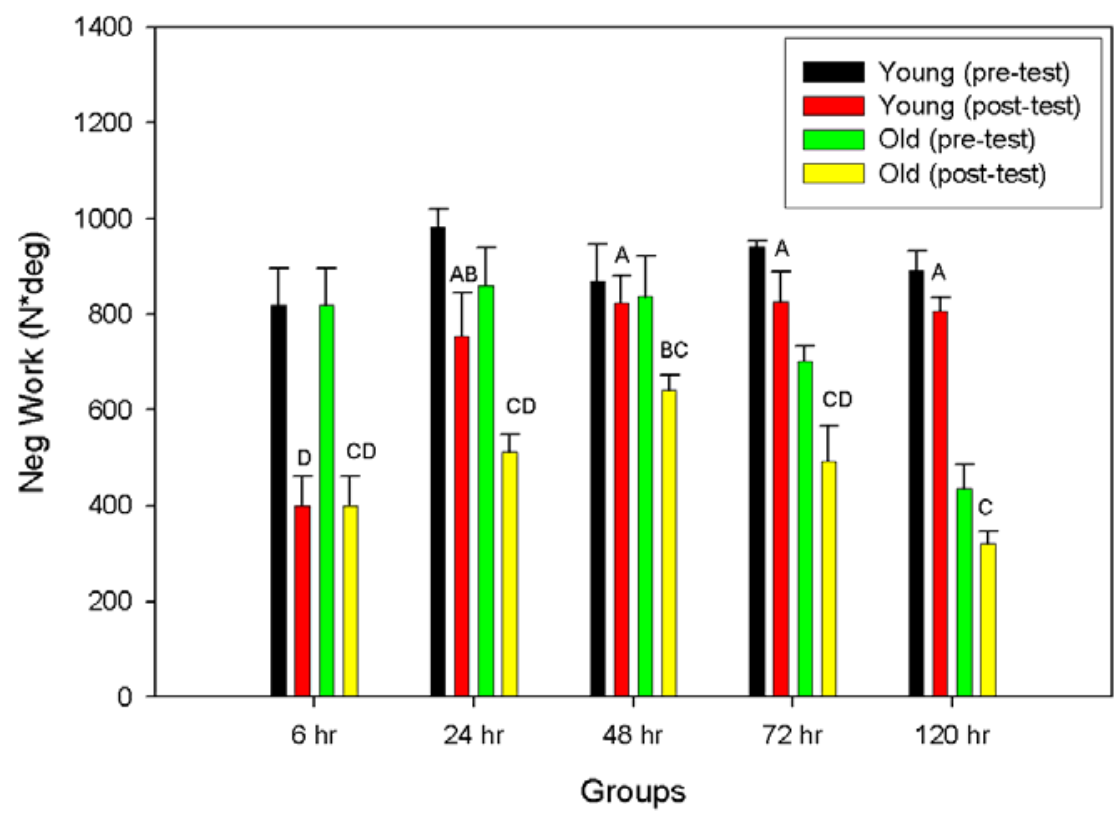

Figure 3b

Positive Work

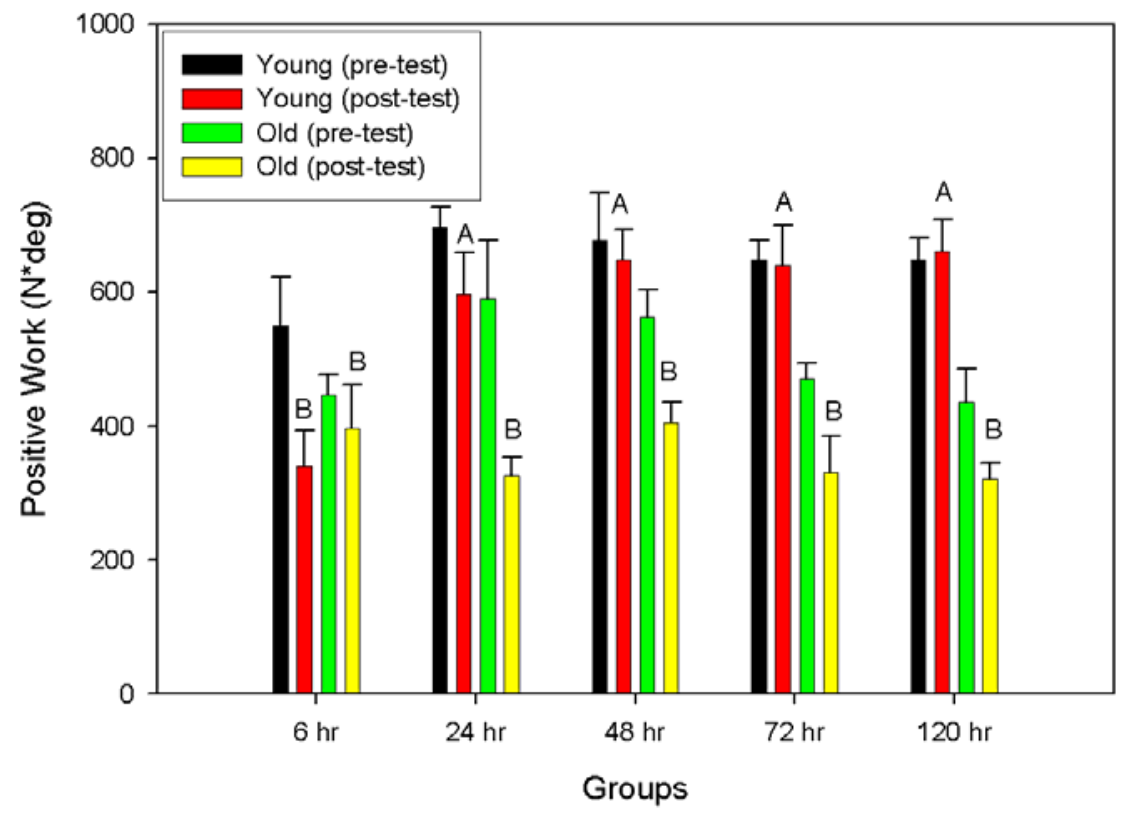


Figure 4

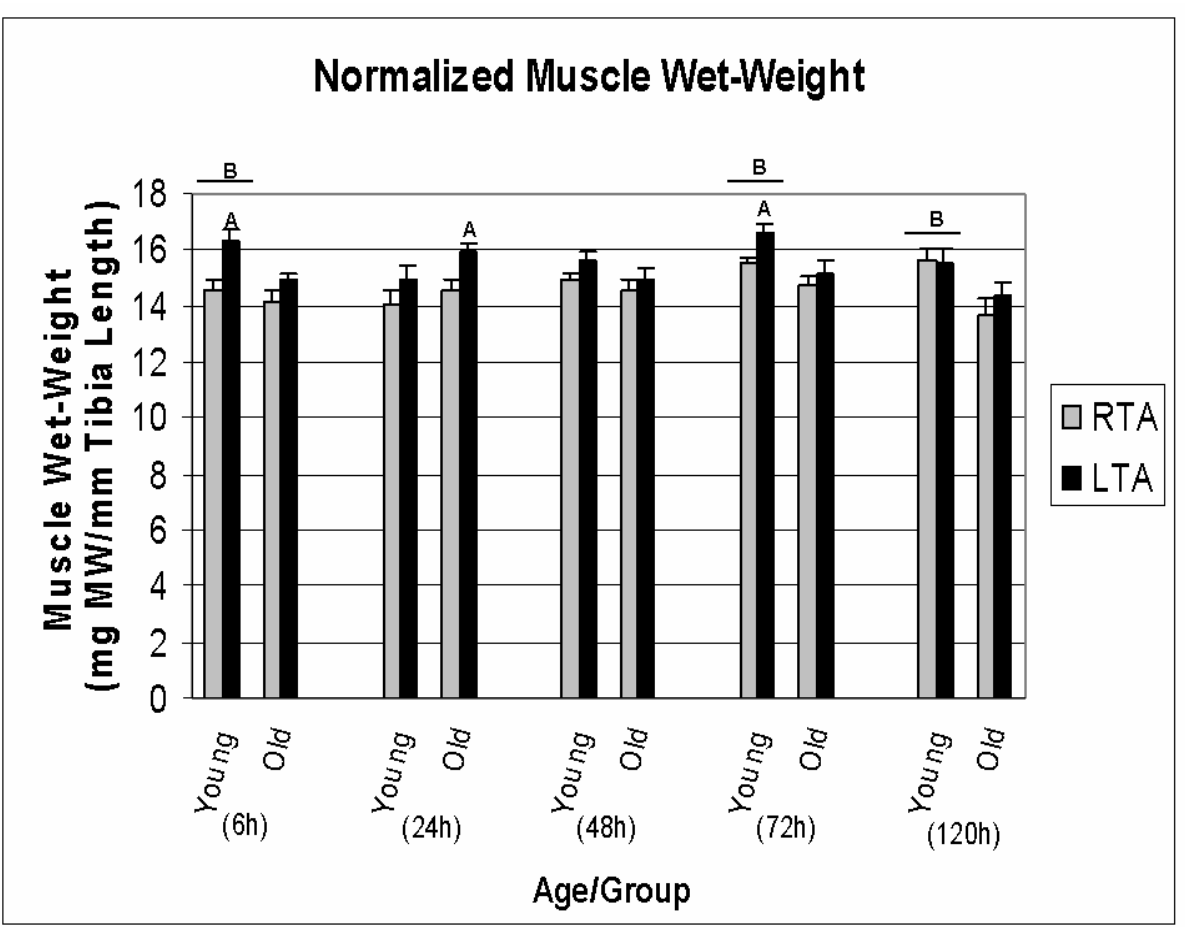


Figure 5a

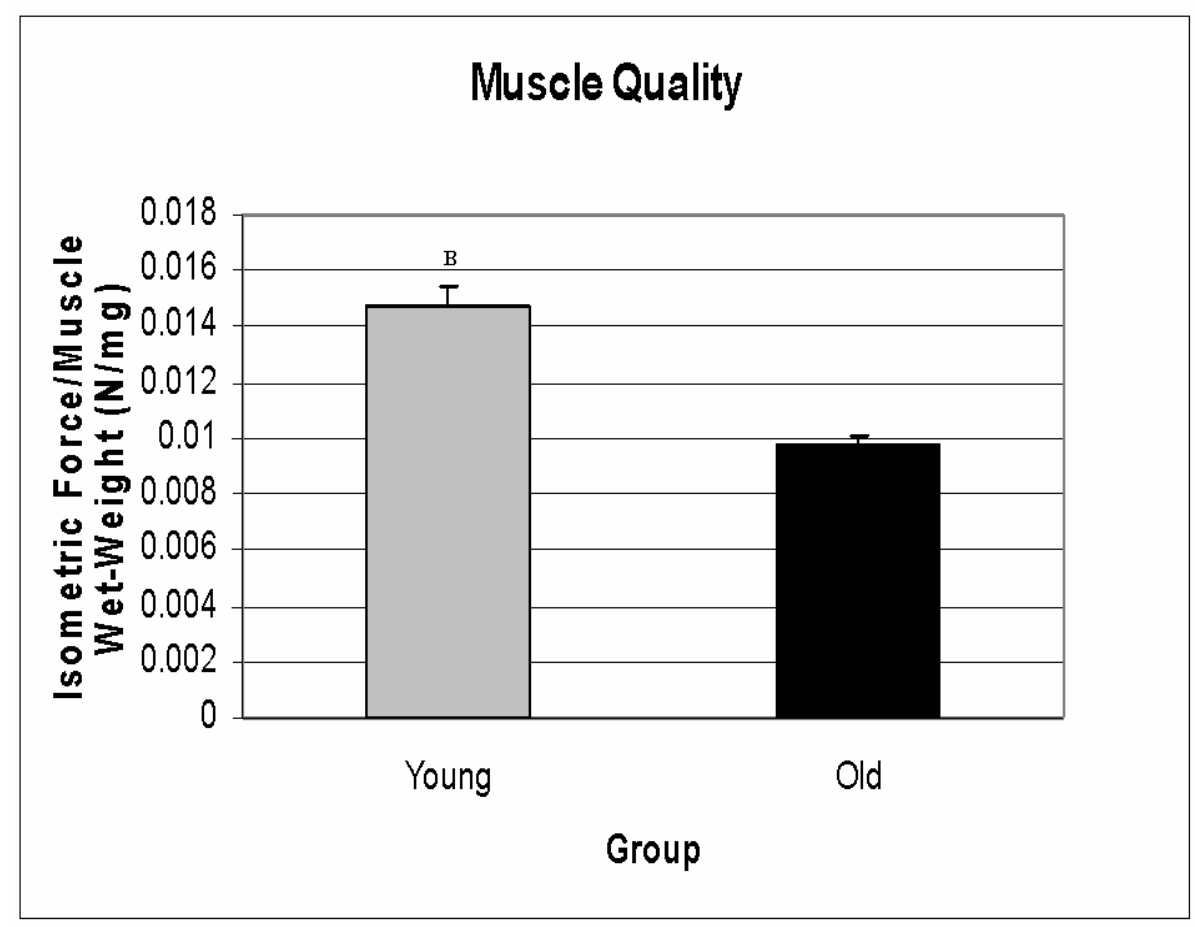

Figure 5b

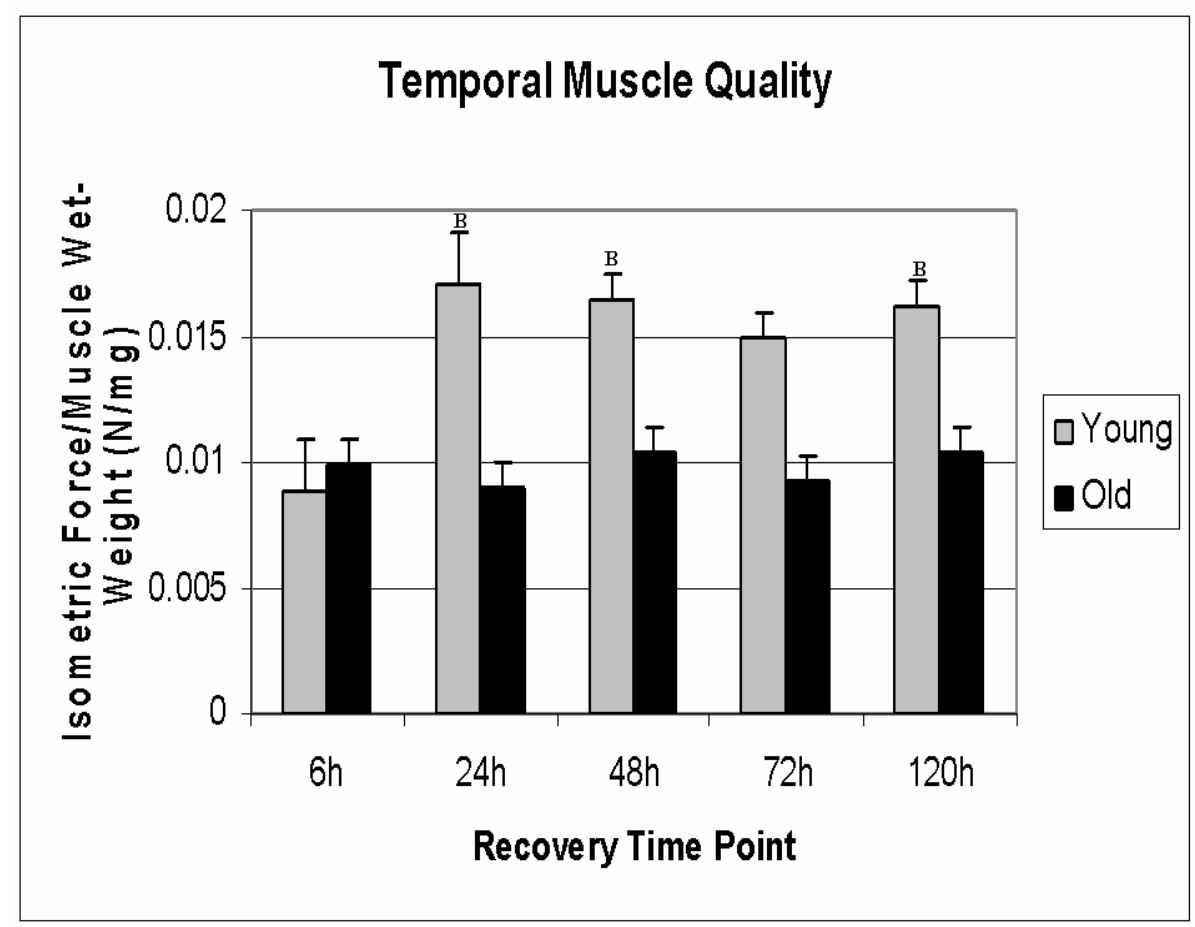


Figure 6
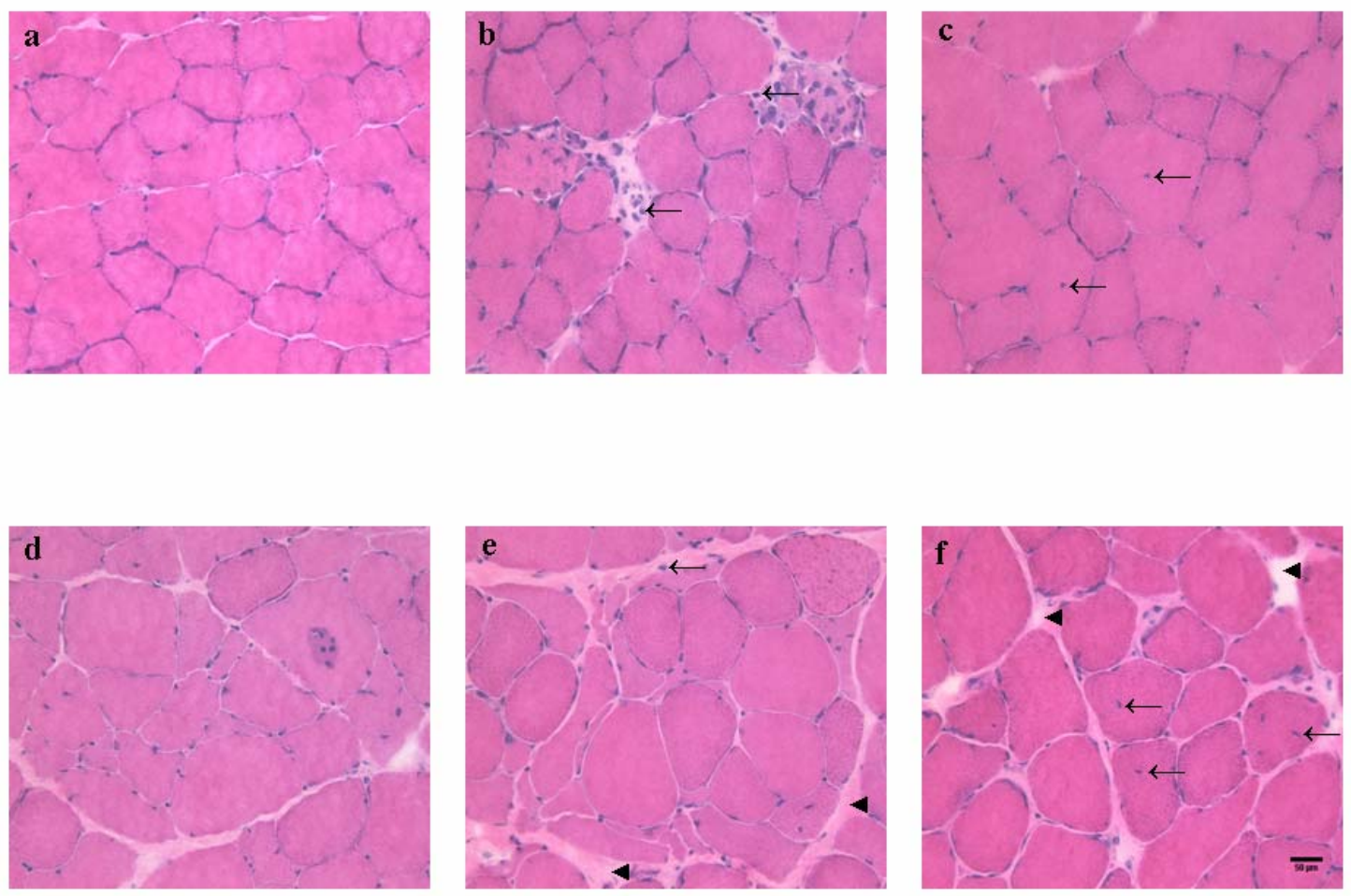
Figure 7a

Acute Exposure-Normal Myofibers

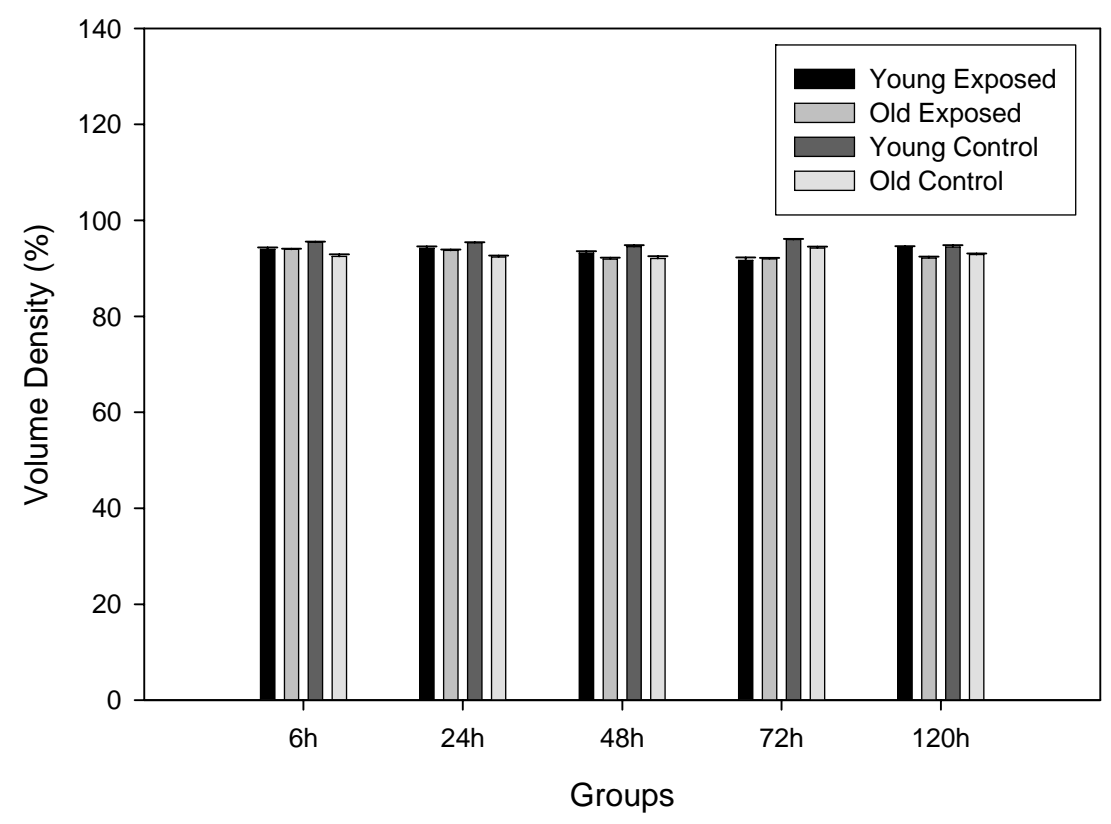

Figure 7b

Acute Exposure-Degenerative Myofibers

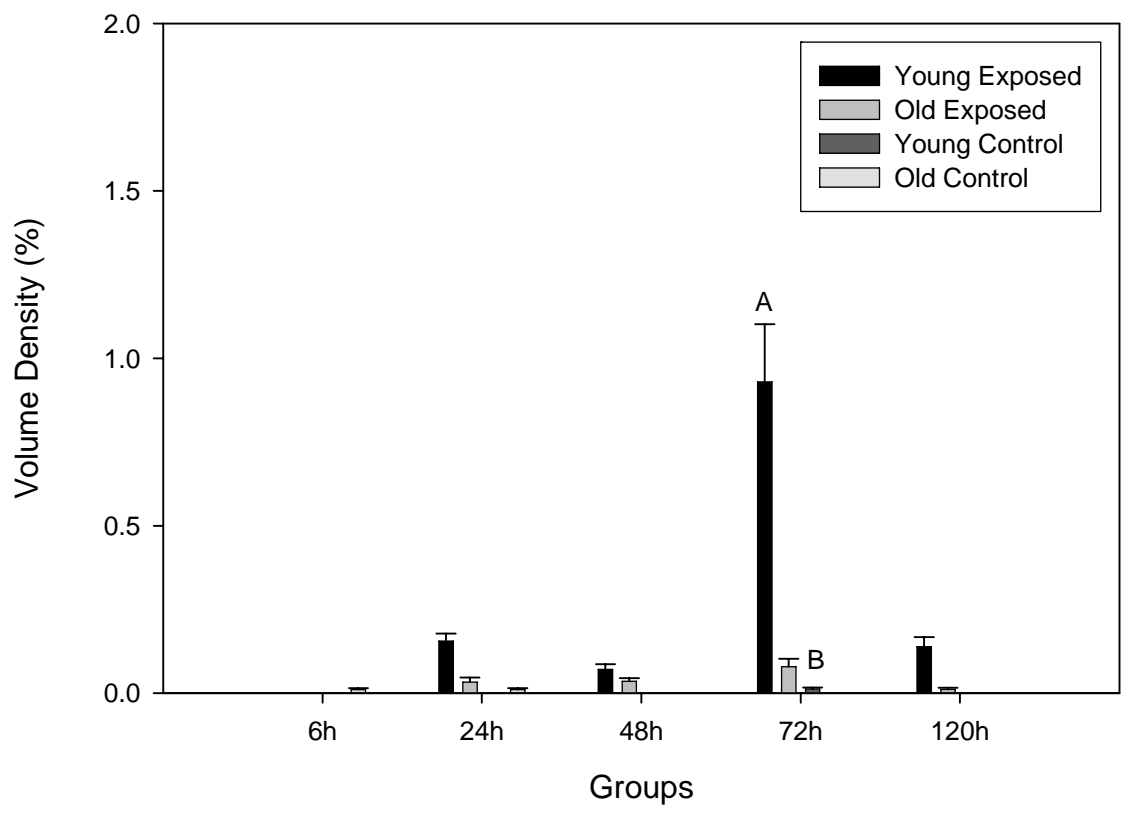




\section{Figure 7c}

Acute Exposure- $\mathrm{NCl}$

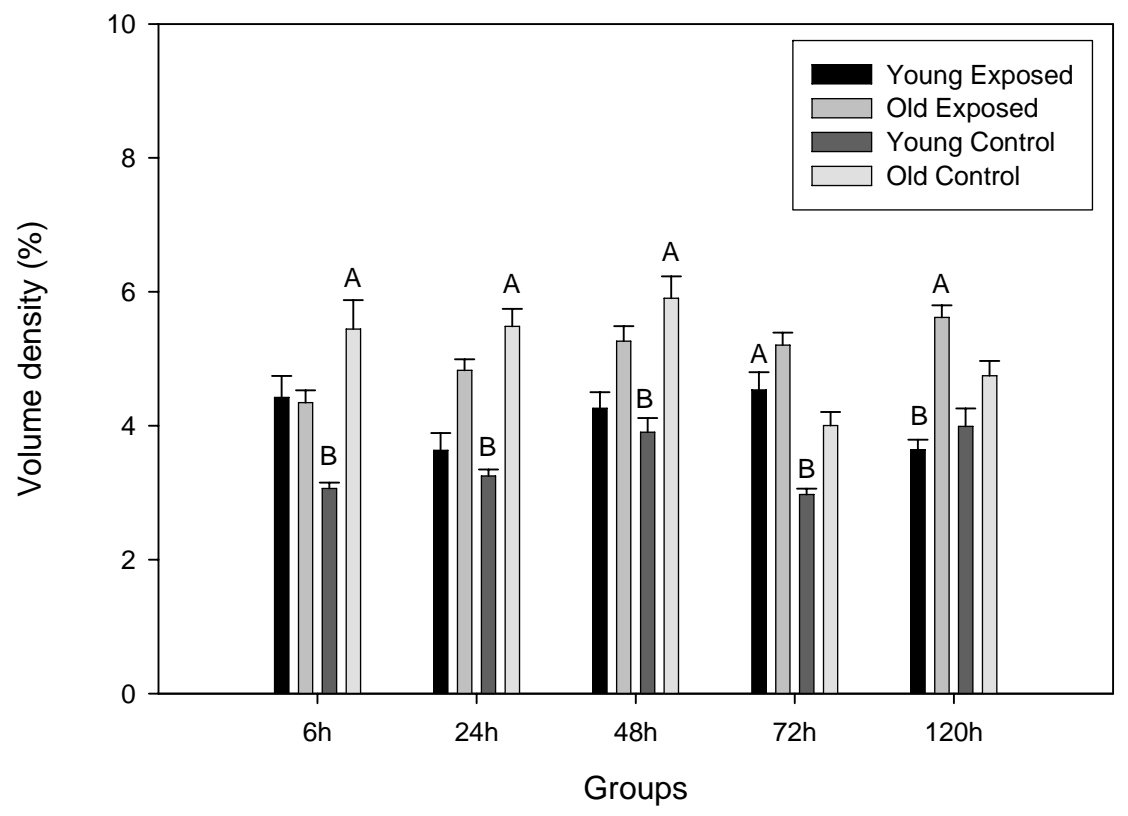

Figure 7d

Acute Exposure-Cl

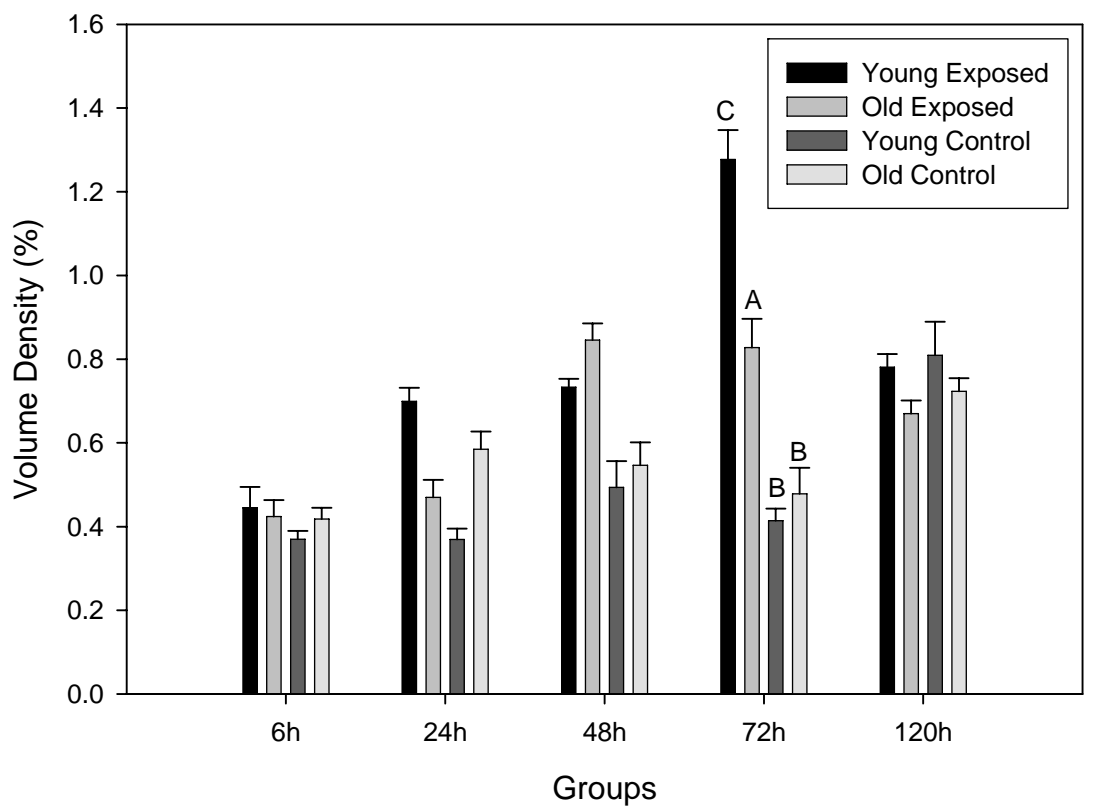


Figure 8a
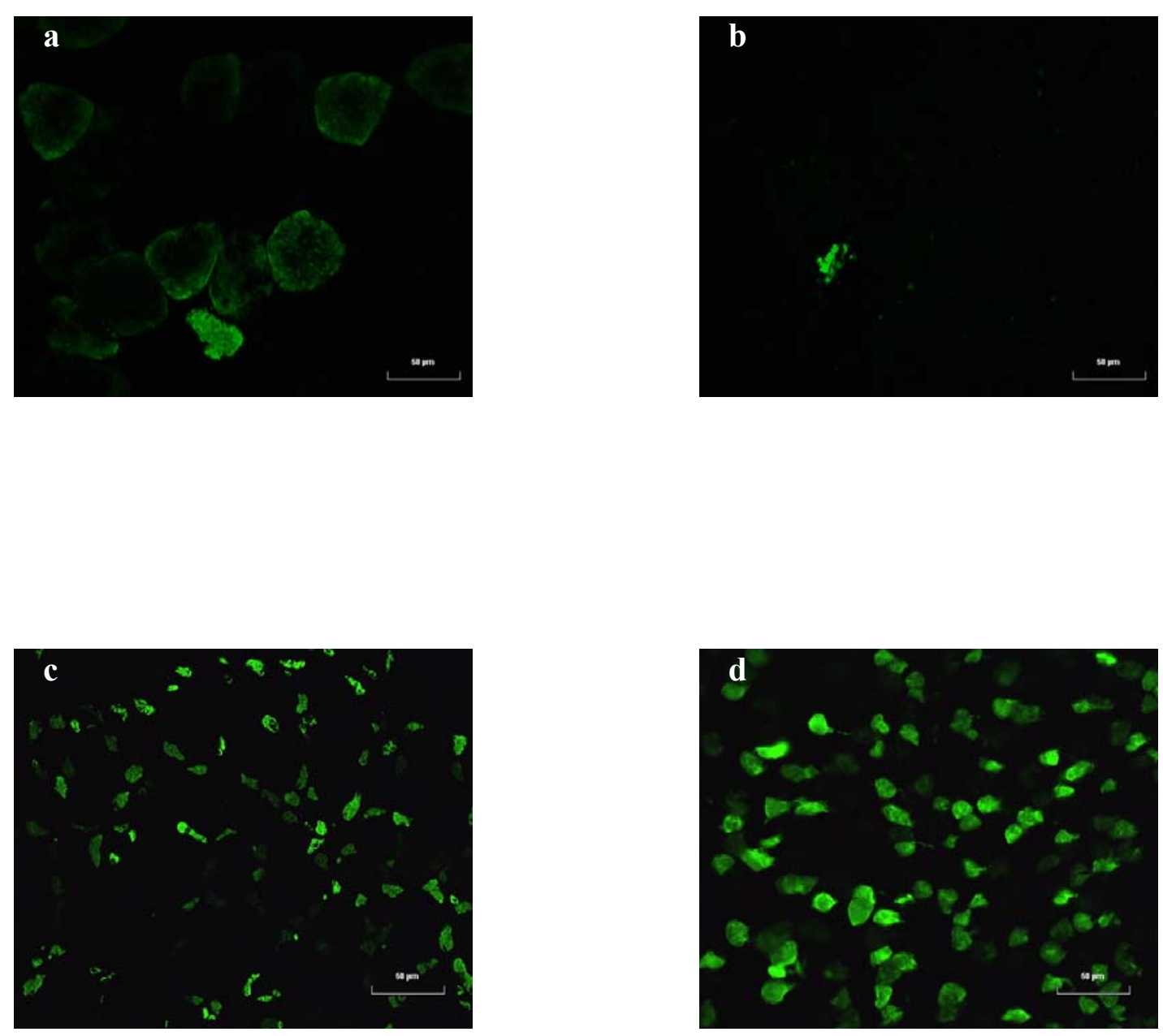
Figure 8b

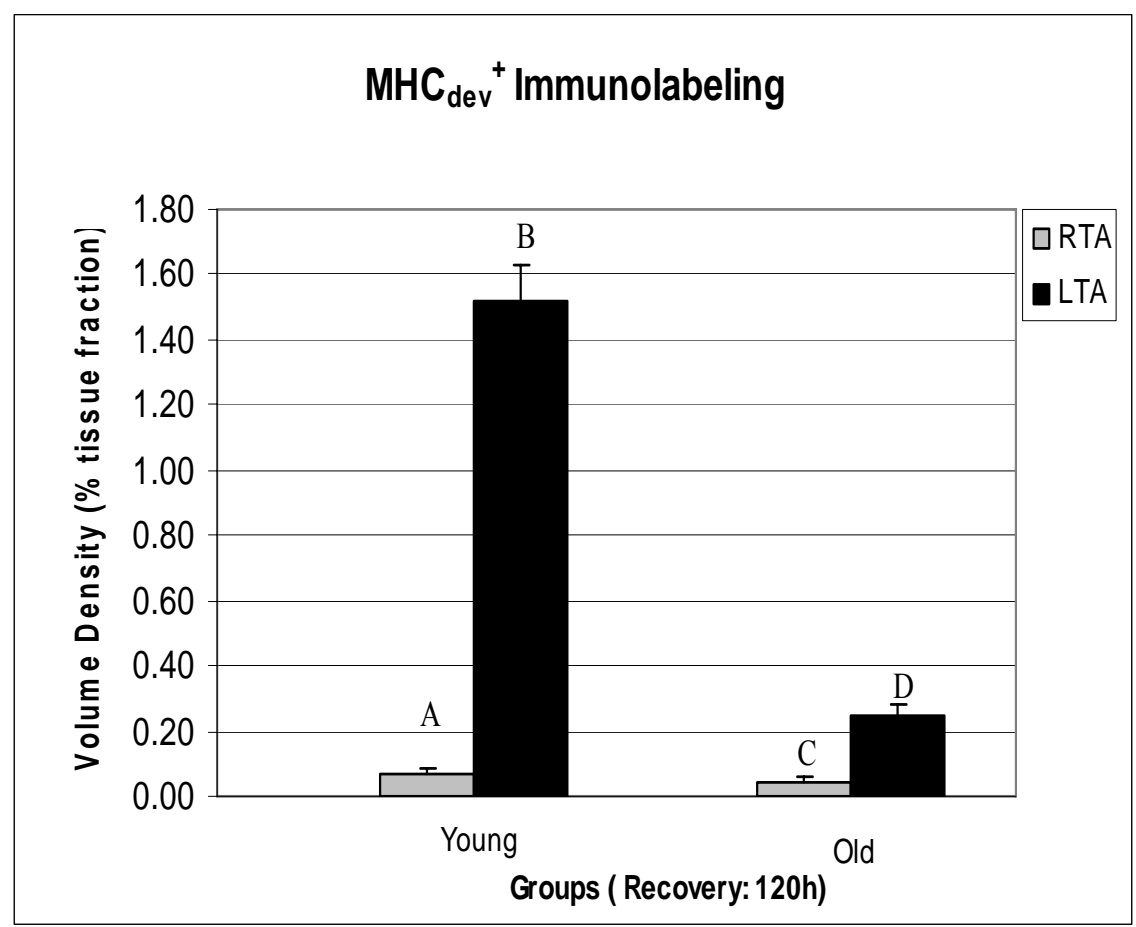




\section{Chapter 4 Amendment:}

The inclusion of this recently published manuscript as part of my dissertation is both necessary (due to this project providing the basis for our subsequent chronic mechanical loading exposures in young and old rodents) and justified (my participation on this project was $100 \%$ - from initiation to completion). 


\section{CHRONIC EXPOSURE TO STRETCH-SHORTENING CONTRACTIONS RESULTS IN SKELETAL MUSCLE ADAPTATION IN YOUNG RATS AND MAL- ADAPTATION IN OLD RATS}

\section{Robert G. Cutlip ${ }^{1}$, Brent A. Baker ${ }^{1}$, Ken B. Geronilla ${ }^{1}$, Robert R. Mercer ${ }^{1}$, Michael L. Kashon ${ }^{1}$, Gerald R. Miller ${ }^{1}$, Zsolt Murlasits ${ }^{2}$ and Stephen E. Alway $^{2}$}

${ }^{1}$ National Institute for Occupational Safety and Health (NIOSH), Health Effects Laboratory Division, Morgantown, WV, 26506, USA. and

${ }^{2}$ Laboratory of Muscle Biology and Sarcopenia, Division of Exercise Physiology, West Virginia University School of Medicine, Morgantown, WV, 26506

Address correspondence to:

Robert G. Cutlip, Ph.D.

National Institute for Occupational Safety and Health Health Effects Laboratory Division

1095 Don Nehlen Drive

$\mathrm{M} / \mathrm{S} 2027$

Morgantown, WV 26505

Fax: (304) 285-6265; Tel: (304) 285-5968; E-mail: rgc8@cdc.gov

Running head: Chronic Stretch-Shortening Cycle Exposure 


\begin{abstract}
Background: Repetitive motion is a major risk factor associated with contraction-induced muscle injury. Aim: The objective of this research was to investigate skeletal muscle response to a chronic administration of stretch-shortening cycles (SSCs) in young and old rats. Research design and methods: Dorsiflexor muscles of old (30 months, $\mathrm{N}=5)$ and young rats (12 weeks, $\mathrm{N}=6$ ) were exposed 3 times per week for 4.5 -weeks to a protocol of 80 maximal SSCs per exposure in vivo. Skeletal muscle response was characterized by isometric and dynamic performance, as well as muscle wet weight and quantitative morphological analyses following the exposure period. Results: The performance of the young and old groups was not statistically different at the start of the exposure. By the end of the exposure, however, a statistical difference was noted as performance increased significantly in the young animals and decreased significantly in the old animals. Muscle wet weight of the left tibialis anterior (TA) in the treated limb was significantly greater in the young compared to old animals $(\mathrm{p}<0.001)$, while there was no difference in the contra-lateral TA. No degenerative myofibers or changes in non-cellular interstitium was noted in either age group, but a significant increase was observed in the volume of the cellular interstitium in the exposed limb of the old animals $(p=0.01)$, which is indicative of an inflammatory response. Conclusions: Thus, a chronic exposure of SSCs results in significant performance increase and muscle hypertrophy in young animals, and a significant performance decrease and an increased cellular interstitial response in old animals. These findings suggest that age may impair the ability of skeletal muscle to adapt to repetitive mechanical loading, even in the absence of degeneration.
\end{abstract}

Keywords: Stretch-shortening cycles, dorsiflexor muscles, repetitive exposure, cellular interstitium 


\section{INTRODUCTION}

Senescent-related changes in strength and skeletal muscle mass have been studied previously (Booth et al., 1994, Evans and Campbell, 1993); however, changes in functional performance and muscle plasticity with training in aged-populations are not fully understood. It is clear that susceptibility to contraction-induced injury increases with age (Bernard et al., 1997) in both humans (Manfredi et al., 1991) and animals (Brooks and Faulkner, 1996, Zerba et al., 1990). After an injurious exposure to eccentric muscle contractions, there is an increased force deficit (Zerba et al., 1990, Brooks and Faulkner, 1996, Koh et al., 2003) and slower recovery of performance (Manfredi et al., 1991, Brooks and Faulkner, 1990, McBride et al., 1995, Sacco and Jones, 1992) in whole muscles (Zerba et al., 1990, Brooks and Faulkner, 1996, Koh et al., 2003, Brooks and Faulkner, 1990, McBride et al., 1995, Sacco and Jones, 1992) and single fibers (Brooks and Faulkner, 1996) of old animals than young animals. The ability to prevent injury in older animals is thought to be attenuated due to higher eccentric force generation during eccentric muscle actions (Brooks and Faulkner, 1994) when compared to young animals, and this difference is magnified with increased stretch velocity (Eddinger et al., 1986). Finally, aging is associated with an impaired adaptation to subsequent exposures of injurious eccentric contractions (McBride et al., 1995).

Few studies have investigated age effects on muscle adaptation and injury following a specific repetitive loading protocol designed to induce hypertrophy. In the case of aerobic training, there is evidence that 10 weeks of treadmill training attenuates eccentric muscle damage in vitro in both young and old rats (Gosselin, 2000). In addition, exposure to 6 weeks of eccentric contractions provided a protective effect in mice by preventing a substantial force deficit and morphological evidence of damage in muscles from both young and old animals 
exposed to a protocol that typically injures non-trained muscles (Brooks et al., 2001). However, older mice adapted more slowly than the young mice (Brooks et al., 2001). While these studies showed that muscles from older animals can be conditioned to be protected from eccentric contraction-induced injury, the conditioning stimulus was inadequate to promote hypertrophy of the target muscles or increase performance. We have previously shown that muscles from old animals are capable of adaptation to increased loads, but aging attenuates loading-induced muscle hypertrophy in rodents (Degens and Alway, 2003, Alway et al., 2002) and birds (Lowe and Alway, 1999, Carson et al., 1995). However, there are no studies to-date that have determined if muscles from old animals can positively adapt to repetitive exposures of resistive muscle contractions that promote adaptation and performance increases in muscles of young animals.

Natural movement is comprised of stretch-shortening cycles (reciprocal concentric and eccentric muscle actions, SSCs), and are an effective means to introduce resistance exercise in skeletal muscle. SSCs have been studied in the context of human locomotion and athletic performance (Avela and Komi, 1998) and have been shown to produce muscle injury due to the eccentric component of the cycle (Horita et al., 1999). Since natural muscle function is comprised of SSCs, this approach provides an improved physiologically relevant exposure model over the traditional eccentric-only injury model (Komi, 2000). The pattern of length changes during SSCs simulates in vivo function more accurately than the ramp stretches typically used (Stevens, 1996).

The purpose of this research was to investigate if aging affects the ability of skeletal muscle to adapt to repetitive exposures of SSCs. Skeletal muscle adaptation was assessed by monitoring the changes in isometric and dynamic performance during the chronic exposure 
period, as well as by morphological changes in skeletal muscle after completion of the SSC protocol. Our general hypothesis is that muscles from older animals have a lower safety threshold or tolerance to repetitive mechanical loading than muscles from young animals. Specifically, we hypothesized that muscles from young animals could adapt to a repetitive mechanical loading protocol that produces hypertrophy, while muscles from older animals would be unable to adapt to the same repetitive loading protocol due to a lower safety threshold or tolerance. Adaptation was defined by a maintenance or increase in contractile performance as a result of the repetitive exposures and the absence of morphological evidence of injury or inflammation. Mal-adaptation was defined as a decrease in contractile performance and the presence of morphological evidence of injury or inflammation as a result of the exposures.

\section{METHODS}

Animal handling: Male Fischer Brown Norway Hybrid rats (F344 x BN F1, N = 11) were obtained from the National Institutes on Aging colony. Young adult $(\mathrm{N}=6,330 \mathrm{~g} \pm 28 \mathrm{~g} \mathrm{SD}, 12$ weeks of age $)$ and old ( $=5,588 \mathrm{~g} \pm 32 \mathrm{~g} \mathrm{SD}, 30$ months) rats were housed in an AAALAC accredited animal quarters. Temperature and light/dark cycle (dark cycle from 7:00 AM to 7:00 PM) were held constant for all animals; food and water were provided ad libitum. After one week of acclimatization, all animals were subjected to a standardized experimental protocol approved by the NIOSH Animal Care and Use Committee.

Experimental setup: The dorsiflexor muscles were tested on a custom-built rodent dynamometer (Cutlip et al., 1997a). The dynamometer provides precise control over the muscle length and muscle force output parameters to be studied. The pennate-fibered dorsiflexor muscle 
group was chosen in our study so that we could directly compare our data to the findings of many previous studies conducted using this muscle group in mice, rats, and rabbits (Benz et al., 1998, Brooks et al., 1995, Cutlip et al., 2004, Davis et al., 2003, Devor and Faulkner, 1999, Faulkner et al., 1989, Geronilla et al., 2003, Lieber and Friden, 1993, Lynch and Faulkner, 1998, Macpherson et al., 1996, Warren et al., 1996).

A Labview-based virtual instrument was developed that governed a National Instruments data acquisition board (PCI-MIO-16XE-10) and Unidex 100 motion controller (Aerotech Inc, Pittsburgh, PA, USA) for precise control of a brushless DC servomotor (1410 DC, Aerotech Inc, Pittsburgh, PA, USA) and muscle stimulator (Model SD9, Grass Medical Instruments, Quincy MA, USA). The software also acquired and stored position, force, and velocity data in real-time as described below.

Rats were anesthetized with $2 \%$ isoflurane gas using a small animal anesthetic system (Surgivet Anesco Inc., Waukesha, WI, USA). Isoflurane was chosen because it has no effect on in vivo force production (Ingalls et al., 1996). After anesthesia, each rat was placed supine on the heated $x-y$ positioning table of the rodent dynamometer, with an anesthetic mask placed over its nose and mouth. The knee was secured in flexion (at $90^{\circ}$ ) with a knee holder. The left foot was secured in the load cell fixture using a custom-built foot holder with the ankle axis (assumed to be between the medial and lateral malleoli) aligned with the axis of rotation of the load cell fixture. Each animal was monitored during the protocol to ensure proper anesthetic depth and body temperature. The animal setup was previously described by Cutlip and colleagues (Cutlip et al., 2004). 
Functional testing: The joint position of the animal was defined by the angle between the tibia and the plantar surface of the foot. The angular position of the load cell fixture corresponded with angular position of the ankle. Vertical forces applied to an aluminum sleeve fitted over the dorsum of the foot were transmitted to a load cell transducer (Sensotec, Inc., Columbus, OH, USA) in the load cell fixture. The force produced by the dorsiflexor muscles was measured at the interface of the aluminum sleeve and the dorsum of the foot. Platinum stimulating electrodes (Grass Medical Instruments, Quincy MA, USA) were placed subcutaneously each exposure session to span the peroneal nerve. Accuracy and reliability of repeated electrode placement was validated via pilot studies measuring isometric forces (coefficient of variation $<9.2 \%$ for 14 repetitive exposures, $\mathrm{N}=6$ ). Activation of the electrical stimulator resulted in muscle contraction of the dorsiflexor muscle group. Stimulator settings were optimized based on pilot studies to maximize dorsiflexor isometric force using a supra-maximal stimulus. Muscle stimulation for all protocols was a $120 \mathrm{~Hz}$ square wave pulse at $0.2 \mathrm{~ms}$ pulse duration, and 4 Volts. To reduce the effect of excitation-contraction fatigue, all electrical stimulation times were kept to a minimum with 2-minutes of recovery time between stimulations (Ingalls et al., 1998).

SSC protocol: The young and old age groups (12 week, $n=6 ; 30$ month, $n=5)$ were exposed to 8 sets of 10 repetitions of SSCs with 2-min intervals between each set. Within each set, there was a 2-s rest between each stretch-shortening contraction (Figure 1a). For each repetition, the dorsiflexor muscles were fully activated for $100 \mathrm{~ms}$ duration via the electrical stimulator, and the eccentric contraction phase was initiated with a $60 \mathrm{deg} / \mathrm{s}$ movement velocity of the load cell fixture over the prescribed range of motion of 90-140 deg ankle angle. The load cell fixture was then immediately returned in the concentric phase at $60 \mathrm{deg} / \mathrm{s}$ to the starting position of $90 \mathrm{deg}$ 
ankle angle. The dorsiflexor muscles were then deactivated $300 \mathrm{~ms}$ later. Total stimulation time per repetition was $2.06 \mathrm{~s}$. The exposure paradigm was designed based on findings from a previous study (Geronilla et al., 2003) that indicated that 70 repetitions of SSCs at a higher velocity $(500 \mathrm{deg} / \mathrm{s})$ produced myofiber injury, thus the repetitions were increased with more rest time between repetitions, and the velocity decreased to $60 \mathrm{deg} / \mathrm{s}$ to promote hypertrophy and adaptation.

Isometric Force Test: A pre-test isometric contraction (pre-test isometric force) was measured on the dorsiflexor muscle group at an ankle angle of 90 deg using a $300 \mathrm{~ms}$ stimulation duration in a similar fashion as Davis et al. (Davis et al., 2003) and Willems and Stauber (Willems and Stauber, 2001). An isometric contraction was also performed immediately following the SSC protocol (post-test isometric force) that consisted of eight sets of ten stretch-shortening contractions.

Single Stretch Shortening Cycle Test: A single stretch-shortening contraction was measured on the dorsiflexor muscle group two minutes preceding and following treatment with the SSC protocol as previously described (Cutlip et al., 2004). This test was used to evaluate the muscle's ability to generate dynamic forces and to perform work during dynamic stretchshortening. The stretch-shortening contraction was performed by activating the dorsiflexor muscles for $300 \mathrm{~ms}$ then moving the load cell fixture from $70-140 \mathrm{deg}$ at an angular velocity of $500 \mathrm{deg} / \mathrm{s}$. The load cell fixture was immediately returned to $70 \mathrm{deg}$, at $500 \mathrm{deg} / \mathrm{s}$ (Figure $1 \mathrm{~b}$ ). Activation was continued for $300 \mathrm{~ms}$ after cessation of the movement. 
Chronic Exposure: The SSC protocol and performance tests were administered three times per week for a total of 14 exposures over a four and a half week period.

Histology: Twenty-four hours after the last exposure, rats were weighed, anesthetized with sodium pentobarbital (ip, $10 \mathrm{mg} / 100 \mathrm{~g}$ body weight) and exsanguinated. The left (exposed LTA) and right (contra-lateral control - RTA) tibialis anterior muscles were dissected and weighed. The midbelly region of the TA was mounted on cork and immersed in optimal cutting temperature medium. Samples were allowed to rest for 3 minutes at room temperature prior to freeze fixation in isopentane cooled in liquid nitrogen and stored at $-80^{\circ} \mathrm{C}$. Transverse frozen sections $(12 \mu \mathrm{m})$ were cut, mounted on pre-coated microscope slides, air dried, and stained using a routine procedure with Harris Hematoxylin and Eosin (H\&E). Tissue sections were viewed using a Leica DMLB microscope.

Muscle quality: Pre-test isometric force measured at the last session of the chronic exposure period was normalized to muscle wet weight of the tibialis anterior of the exposed limb obtained at sacrifice as previously described (Degens and Alway, 2003).

Fiber cross-sectional area: For muscle fiber cross-sectional area (CSA) analysis, ten nonoverlapping digital images were obtained from hematoxylin and eosin-stained muscle sections at a 40X magnification and, subsequently, were analyzed for tibialis anterior fiber CSA $(\mu \mathrm{m} 2)$ with National Institutes of Health imaging software (ImageJ). Each fiber was traced with a handheld mouse, and the number of pixels traced was calibrated to a defined area in square micrometers. Approximately 200 fibers were traced per sample. Preliminary studies have shown that a sample 
size of 200 fibers was adequate because this sample number had no change in the standard deviation or the mean of the fiber CSA as compared with a higher number of fibers sampled (data not shown).

Stereology: Quantitative morphometric methods were used to measure the volume fraction, surface densities, and average thickness of normal myofibers, degenerative myofibers, and the interstitial space (Baker et al., in press-b). The interstitium was divided into endomysium and perimysium spaces, which included capillaries. A standardized stereological technique, as previously discussed (Baker et al., in press-b), was used to quantify the degree of myofiber degeneration and inflammation, which was quantified as either non-cellular interstitium (NCI), indicative of edema, or cellular interstitium (CI), indicative of cellular infiltrates. Fiber volume and surface density were measured using standard morphometric analyses (Underwood, 1970, Weibel, 1972, Weibel, 1974, Weibel, 1975). Briefly, one of the H\&E stained sections was taken from each animal. A stage micrometer was used to identify the mid-point of the section. Point and intercept counts using a 121-point / 11-line overlay graticule (12.5 mm square with 100 divisions) at 40x magnification were taken at 5 equally spaced points across the section. This process was repeated $2 \mathrm{~mm}$ on either side of the mid-point of the section for a total of 1210 points and 110 intercept lines per section. Volume density or percent volume was computed from the percentage of points over the tissue section to points over normal myofibers, degenerative myofibers, cellular interstitium and non-cellular interstitium plus capillaries (Weibel, 1972, Weibel, 1974, Weibel, 1975). Intercepts over the line overlay were counted for the perimeter of normal myofibers, degenerative myofibers, and interstitium to myofiber transitions. Points and intercepts over blood vessels greater than 25 microns $(\mu \mathrm{m})$ in diameter 
were excluded. Average thickness or average distance was computed from two times the ratio of volume to surface density according to standard morphometric analysis (Underwood, 1970). One section per animal with an $\mathrm{n}=6$ for young and an $\mathrm{n}=5$ for old animals per group was evaluated and the results expressed as mean \pm S.E.M. Stereology was used to quantify the degree of myofiber degeneration, and the accompanying changes in the TA muscle from each group. Myofibers were defined by the following criteria: Normal myofibers demonstrated: 1) Complete contact with adjacent myofibers, 2) A smooth outer membrane, and 3) No presence of internal inflammatory cells. Degenerative myofibers displayed: 1) A loss of contact with adjacent myofibers, 2) Presence of internal inflammatory cells, and 3) An outer membrane interdigitated with inflammatory cells.

\section{DATA ANALYSIS}

Single Stretch-Shortening Cycle: The experimental data from each single SSC was used to calculate the group means and standard error of the mean for $\mathrm{F}_{\text {peak }}$ (peak eccentric force), $\mathrm{F}_{\text {min }}$ (isometric pre-stretch force), negative work, and positive work. These force and work parameters were calculated for all 14 exposures over the 4.5 week exposure period. Work was calculated in the same fashion as previous work by Cutlip and colleagues (Cutlip et al., 2004), Ettema (Ettema, 1996), Stevens and Faulkner (Stevens and Faulkner, 2000), and Stevens (Stevens, 1996). The dynamic force parameters $F_{\text {peak }}$, and $F_{\min }$, were calculated in the same fashion as previous work by Cutlip and colleagues (Cutlip et al., 2004), and Geronilla and colleagues (Geronilla et al., 2003). The data analysis for this study was generated using SAS/STAT software, Version 8.2 of the SAS System for Windows (SAS Institute, Cary, NC). Isometric force, dynamic forces, and work measures were analyzed using the mixed model 
analyses of variance (age by day) with repeated measures on day with Proc Mixed. A spatial power covariance structure was used for the repeated measure aspect to appropriately model the spacing of the exposures. Stereological measures were analyzed using a factorial (age by limb) mixed model analysis of variance with animal as the random factor to account for measures in both limbs. Post hoc comparisons between groups at each exposure were analyzed using the slice option in SAS.

Muscle weights: Data for muscle weight differences between the exposed muscle and control muscle were analyzed using a two-sample t-test procedure assuming unequal variances.

Stereology: For the stereology the statistical analyses were conducted using SAS Version 8 (SAS Institute, Cary, NC). Stereological measurements for volume and thickness of cellular and noncellular components and muscle quality were analyzed using two-way mixed model (age x limb) ANOVAs with the animal as the random factor accounting for measures in both limbs.

\section{RESULTS}

\section{Functional performance}

Isometric force: The pre-test isometric force response over the chronic exposure period was different between the old and young age groups $(p=0.0003$, Figure 2$)$. Despite the age difference, the young and old animals generated very similar magnitudes of isometric force on the first day of exposure $(\mathrm{p}=0.455$, Figure 2$)$. However, the isometric forces between the two groups started to diverge by day 8 (fourth exposure) of the exposure period $(p=0.045$, Figure 2 ) and continued to diverge throughout the exposure period, resulting in a substantial difference in 
isometric force generated by the two age groups by the end of the thirty day exposure period (fourteenth exposure, $p<0.0001$, Figure 2). The pre-isometric test showed a $25 \%$ increase in isometric force for the young group after 4.5-weeks, while the old group had a decrement of $34 \%$.

Peak force ( $\left.\mathbf{F}_{\text {peak }}\right)$ : The peak eccentric force response over the chronic exposure period was also quite different for the two age groups $(p=0.0081$, Figure $3 a)$. The young and old animals produced similar $\mathrm{F}_{\text {peak }}$ forces during the pre-exposure $\mathrm{SSC}$ on the first day of exposure $(\mathrm{p}=$ 0.761 , Figure $3 a$ ) but began to diverge by day 15 (seventh exposure, $p=0.013$, Figure $3 a$ ). The $\mathrm{F}_{\text {peak }}$ generated by the two age groups continued to diverge throughout the remainder of the exposure period which resulted in an increase of $27.9 \%$ in the young group and a decrease by $22.4 \%$ in the old group by the end of the 4.5 week exposure period $(p<0.0001$, Figure $3 a)$.

Minimum force $\left(\mathbf{F}_{\mathbf{m i n}}\right)$ : The minimum force response over the chronic exposure period was similar to the peak force and isometric force responses $(\mathrm{p}=0.0011$, Figure $3 b)$. The two age groups produced similar $\mathrm{F}_{\min }$ forces during the single stretch-shortening cycle on the first day of exposure $(\mathrm{p}=0.853$, Figure $3 \mathrm{~b}$ ) but began to diverge by day 19 (ninth exposure, $\mathrm{p}=0.002$, Figure $3 b$ ). The $F_{\min }$ generated by the two age groups continued to diverge throughout the remainder of the exposure period much like the $\mathrm{F}_{\text {peak }}$ and isometric force, thus resulting in an increase in $\mathrm{F}_{\min }$ by $25.9 \%$ in the young group and decrease of $32.5 \%$ in the old group by the end of the 4.5 week exposure period ( $p<0.0001$, Figure $3 b)$. 
Work: The age of the animal also influenced the ability of the dorsiflexor muscles to both absorb work (negative work) and produce work (positive work). The two age groups exhibited the same ability to absorb work during the first exposure $(p=0.455$, Figure $4 a)$. However, the ability to absorb work differed between age groups with subsequent exposures during the chronic exposure period $(p=0.0044$, Figure $4 a)$. Negative work increased by $27.0 \%$ in the young group while negative work decreased by $24.9 \%$ in the old group, resulting in a significant difference by the end of the chronic exposure period $(p<0.0011$, Figure $4 a)$. The change in response became significantly different between the old and young groups on day 15 (exposure 7) of the chronic exposure $(p=0.0063$, Figure $4 a)$.

The two age groups also exhibited the same ability to produce positive work during the first exposure $(p=0.475$, Figure $4 b)$. However, in a similar fashion as negative work, the ability to produce positive work differed between age groups with subsequent exposures during the chronic exposure period $(\mathrm{p}=0.0011$, Figure $4 \mathrm{~b}$ ). Positive work increased by $26.5 \%$ in the young group, while positive work decreased by $39.2 \%$ in the old group, resulting in a substantial difference by the end of the chronic exposure period ( $p<0.0011$, Figure $4 b)$. A significant change in the response was observed between the old and young groups on day 15 (exposure 7) of the chronic exposure $(p=0.016$, Figure $4 b)$.

Muscle wet weights: Weights of the exposed muscles and contra-lateral control muscles were normalized to the body weight at the end of the exposure period as shown in Figure 5. Following 4.5 weeks of SSC contractions, the wet-weight of the exposed muscle from young animals was significantly increased when compared to the contra-lateral control muscles $(\mathrm{p}<0.001)$. However, there was no difference between the exposed muscle and control muscle 
wet-weight in old animals after the experimental intervention. Further analyses revealed a significant increase in the wet-weight of exposed muscles in young animals when compared to the exposed muscles in old animals $(\mathrm{p}<0.001)$. In addition, there was a significant difference between the young contra-lateral control and the old contra-lateral control muscle weight $(\mathrm{p}<0.001)$.

Fiber Cross-sectional area: Review of the histological sections is supportive of the muscle wet weight data. Representative sections from the young exposed LTA (Figure 6a) depict normal morphology and larger fibers than the young non-exercised RTA (Figure 6b), indicative of a hypertrophic response. However, the muscle sections from the old exercised LTA (Figure 6c) depicts cellular infiltrates, and central nucleated fibers that are not evident in the samples from the non-exercised limb in either the old animals (Figure 6d) or young animals (Figures 6a and b). In the contra-lateral control muscle (RTA), approximately $45 \%$ of the fibers from the old animals and $54 \%$ of the fibers from the young animals were $\geq 1500 \mathrm{um}^{2}$ (Figure 7). In the exposed limb (LTA), approximately $46 \%$ of the fibers from the old animals and $76 \%$ of the fibers from the young animals were $\geq 1500 \mathrm{um}^{2}$ (Figure 8). This indicates that exposure to chronic SSCs resulted in an increased percentage of larger fibers in the young animals (from 54\% to $76 \%$ ) without a corresponding shift to larger fibers in the older animals ( $45 \%$ to $46 \%$ ).

Body weights: The average body weight of the young group was 331 grams at the start of the study and increased by and average of 3.3\% over the 4.5 week protocol. The average body weight of the old group was 588 grams at the start of the study and decreased by an average of $11.2 \%$ over the 4.5 week exposure. 
Muscle Quality: The mean value of isometric force normalized to muscle wet weight was 0.017 $\pm 0.001 \mathrm{~N} / \mathrm{mg}$ and $0.010 \pm 0.0009 \mathrm{~N} / \mathrm{mg}$ for the young group and old group, respectively (Figure 9). The young group had a significantly greater isometric force normalized to muscle wet weight in the exercised limb after the chronic SSC protocol than the old group ( $p=0.001$, Figure 9).

Stereological analyses of myofiber degeneration: Statistical analysis showed that there were no degenerative myofibers present in muscle samples analyzed from either the young or old animals.

Stereological analyses of inflammation: We observe no change in NCI as a result of the repetitive loading intervention in either age group which suggests that there was no edema present. Nevertheless, there was a significant effect of age $(p=0.02)$ for the volume density of CI. The volume density of CI in the exposed muscles was significantly greater in old as compared to young animals $(\mathrm{p}=0.01$, Figure 10$)$. Furthermore, the volume of CI was significantly higher in the exposed muscles when compared to the contra-lateral control muscles of old animals ( $\mathrm{p}=0.05$, Figure 10). Also, volume density of CI was significantly higher in the exposed muscles of old animals when compared to the control muscles of young animals $(\mathrm{p}=0.01$, Figure 10).

\section{DISCUSSION}

The major finding of this study is that the dorsiflexor muscles from young animals adapt functionally to 4.5-weeks of SSC exposure, whereas the same SSC protocol results in functional mal-adaptation in muscles from old animals. This maladaptation in old animals does not appear 
to be the result of necrotic myofibers due to an absence of morphological markers of myofiber degeneration. However, the exposed muscles of old animals did exhibit an increased cellular interstitium response, indicative of inflammation, suggesting the myofibers may have been under stress but not to the level of necrosis.

The observation that the functional performance of both the young and old animals was similar at the start of the exposure period is critical in acknowledging, because this establishes that age did not have an affect on the ability of untrained animals to generate force at the onset of the exposure protocol. Additionally, isometric performance and dynamic performance, as indicated by isometric pre-stretch forces $\left(\mathrm{F}_{\text {min }}\right)$ and peak eccentric forces $\left(\mathrm{F}_{\text {peak }}\right)$ during the single SSC, as well as negative and positive work absorbed and produced during the SSC, were not different at the start of the exposure. This is consistent with Brooks et al. (Brooks and Faulkner, 1994) who reported no difference in concentric performance in old and young animals or in isometric performance in soleus muscles of mice. There was, however, evidence of lower forces in the TA of older animals when normalized for muscle dry weight (Brooks and Faulkner, 1988). Brooks and colleagues (Brooks et al., 2001) also showed no initial difference in isometric performance of the TA or extensor digitorum longus (EDL) muscles in adult versus old mice. In earlier studies, a single exposure to injurious eccentric exercise resulted in similar isometric force decrements regardless of age in mice (Brooks and Faulkner, 1996, Zerba and Faulkner, 1990, Koh et al., 2003) and rats (Gosselin, 2000) but older animals can exhibit larger force deficits with more severe exposures (Zerba et al., 1990). However, McBride et al. showed that muscle from adult animals exposed to damaging eccentric contractions in situ exhibit isometric force recovery 14 days after the exposure, while muscles from old animals did not (McBride et al., 1995). Our data are consistent with these findings in that old animals appear 
unable to recover from repeated bouts of SSCs as evidenced by their reduced force generating capacity.

Although aging muscles have a delayed recovery from a single injurious exposure, these muscles do retain some ability to adapt. For example, when damaging eccentric muscle actions are incorporated into a repetitive exposure model, there is evidence that age does not have a deleterious effect on adaptation when this eccentric protocol is administered once per week in vivo (Brooks et al., 2001). In support of this, adult and old mice exhibited similar isometric and peak force deficits of the dorsiflexors after the initial exposure, and the recovery of those forces in rest periods between bouts of exposures was also similar (Brooks et al., 2001). Moreover, following 6 weeks of conditioning, the maximal isometric force was not different between age groups. In spite of this, the amount of rest between exposures can affect the ability to adapt to potentially beneficial and/or injurious contractions, particularly in aged animals. The exposure paradigm used in the current study resulted in an average increase in force of $25 \%$ above the pretest force in young animals (indicative of adaptation), while producing a $34 \%$ deficit in old animals (indicative of mal-adaptation). The peak eccentric force also increased approximately $28 \%$ in young animals, while decreasing $22.4 \%$ in the old animals. In contrast, Brooks et al. (Brooks et al., 2001) reported a significant increase in peak force by week 6 for both adult and old animals over baseline values, however this was reported in mice. Thus, a differential effect may exist between species as well as the previously noted difference in rest between exposures.

To our knowledge, this is the first study to report that changes due to repetitive SSC resulted in a significant increase in negative and positive work in young animals over baseline values that was commensurate with increases in isometric force and peak eccentric forces. Conversely, the protocol produced significant decreases in negative and positive work in the old 
animals as compared to baseline values that was commensurate with losses in isometric force and peak eccentric forces. The results from the repetitive loading model of Brooks et al. (Brooks et al., 2001) indicated that older animals require more time to adapt to injurious muscle contractions as assessed by a force decrement after exposure. Our results are consistent with this observation, since as exposure to injurious contractions is repetitively administered; older animals were less able to adapt.

The morphological changes that occurred during the chronic exposure period were quite different between the two age groups. The exposed muscle mass in young animals was $\sim 17 \%$ greater than the contra-lateral control muscle after 4.5 weeks of repetitive loading. This corresponds well with the magnitude of hypertrophy in young animals reported in previous work in which dorsiflexor muscles from young male mice were exposed to a 6-week conditioning program (Faulkner et al., 1992). Also during 10-week training protocol, the TA muscle of rats exhibited a $16-30 \%$ increase in muscle mass compared to the contra-lateral limb (Wong and Booth, 1990). Moreover, a 6-week conditioning program in dorsiflexor muscles of female mice resulted in protection from contraction-induced injury in both old and young animals, despite no measurable hypertrophy. Brooks et al. (Brooks et al., 2001) hypothesized that this must be due to intrinsic strengthening of the sarcomeres within the myofibrils via regeneration of stronger sarcomeres, which are more resistant to injury. Earlier work conducted by Devor and Faulkner support this hypothesis (Devor and Faulkner, 1999). In our study, performance significantly declined over the 4.5-week exposure period in the old animals, even though eccentric contractions were incorporated in the exposure paradigm.

In our study, stereological analyses detected no degenerative myofibers or indications of edema following the terminal session, even though there was a significant increase in the volume 
of CI, indicative of inflammation, in the exposed muscles when compared to the contra-lateral limb. This absence of degenerative myofibers, or necrotic fibers, is in apparent contrast to previous studies of acute exposure to eccentric contractions (Faulkner et al., 1989, Koh et al., 2003, Van Der Meulen et al., 1997, Devor and Faulkner, 1999, Lieber and Friden, 1993), and SSC exposure (Geronilla et al., 2003), where necrotic fibers have been reported. Moreover, this absence of degenerative myofibers in old animals that exhibited a significant decrement in functional performance is a novel finding, which needs further exploration regarding the causal mechanisms. Thus, an important factor involving the remodeling of skeletal muscle during adaptation is the modifications made to the interstitial space. This is not only accomplished through interactions with the myofiber (muscle specific factors), but, also, through coordinated interaction with the CI (systemic, non-muscle specific factors). Therefore, the inability of old animals to adapt may be more influenced by the interstitial space and extrinsic environment (Conboy et al., 2005).

The data in the present study clearly show that exposed muscles in young rats adapt to the chronic SSC exposure by increasing muscle mass. The increase in muscle wet-weight could have resulted from and be attributed to chronic edema, but based on our findings, we do not believe this is the case. Results from our muscle cross-sectional area data support the observation that there was a substantial degree of myofiber hypertrophy in the young, exposed animals as evidenced by a shift to larger fibers. In addition, stereological analysis of exposed muscle from young rats showed no increases in NCI response, (i.e., no edema). The observed increase in CI response, indicative of inflammation, may have prevented the decline in musclewet weight that would have been expected by the decline in muscle force and power. However, the observation that old animals did not show signs of degeneration supports the hypothesis 
established by Conboy and Rando that there is at least a minimal regenerative potential (Conboy et al., 2005) as indicated by the maintenance of normal myofibers, which is preserved with aging. Presumably, this is accomplished with modifications to the interstitial space via signals to muscle satellite cells (Conboy et al., 2005). Despite previous reports that indicate skeletal muscles in old animals are more susceptible to injury (Brooks and Faulkner, 1996, Zerba et al., 1990), and recover more slowly (McBride et al., 1995, Brooks and Faulkner, 1990) from a single exposure to injurious contractions, in support of our findings is evidence that old animals can be conditioned for protection from contraction-induced myofiber injury (Brooks et al., 2001). Muscle hypertrophy and improvements in force production occurs in response to constant or chronic loading in aged animals, although the extent of muscle enlargement is attenuated relative to young animals (Alway, 1995, Alway et al., 2002, Carson et al., 1995, Klitgaard et al., 1989a, Klitgaard et al., 1989b, Lowe et al., 1998). However, in our current study the frequency and/or intensity of exposures likely exceeded the ability to recover from the preceding SSC exposure in muscles from old animals, thus exceeding their safety threshold or tolerance.

In addition to age, several factors may explain the difference in performance between the two groups during the chronic protocol. The older animals may not have tolerated the repeated exposure to isoflurane as well as their younger counterparts which could have affected contractile performance. Also, there may have been differences in excitation-contraction coupling or muscle activation sensitivity between age groups as the chronic protocol progressed. Myofiber cross sectional area and stereology data from the contralateral limbs of both groups were not different, which suggests that there was not a different systemic response. The lack of myofiber degeneration in the exposed limbs of the old group suggests that the loss of function is not due to fiber necrosis. Further studies are needed to delineate the contributions of repeated 
anesthesia and multiple exposures of SSCs. Reducing the number of exposures per week may allow the older animals to adapt to the repetitive exposures. Titrating the number of exposures per week and number of repetitions per exposure to further explore the safety threshold of aged skeletal muscle is needed.

Our findings indicate that the frequency of exposures may have profound implications on the ability to adapt to repetitive SSCs. Identifying the safety threshold of skeletal muscle under repeated loading conditions and how it is affected by age, physical training, and lifestyle (e.g. diet, supplementation), is of major importance when designing preventative strategies in vocational and recreation arenas, as well as for understanding the etiology of repetitive loading injuries. In the present study, we show concurrent adaptation in young animals and maladaptation in old animals in the absence of myofiber degeneration. This strongly suggests that there is a level of exposure where the ability to adapt to mechanical exposure is severely compromised by age (Degens and Alway, 2003). Whereas muscle strength may be maintained and, in some instances, enhanced in senescence via resistive loading, the frequency of loading may play a key role in the ability of skeletal muscle to adapt in elderly populations. Although identifying the mechanisms responsible for the mal-adaptation to repetitive loading in aged muscles is beyond the scope of this study, further investigation is warranted. 


\section{REFERENCES}

ALWAY, S. E. (1995) Slowing of contractile properties in quail skeletal muscle with aging. $J$ Gerontol A Biol Sci Med Sci, 50A, B26-33.

ALWAY, S. E., DEGENS, H., KRISHNAMURTHY, G. \& SMITH, C. A. (2002) Potential role for Id myogenic repressors in apoptosis and attenuation of hypertrophy in muscles of aged rats. Am J Physiol Cell Physiol, 283, C66-76.

AVELA, J. \& KOMI, P. V. (1998) Reduced stretch reflex sensitivity and muscle stiffness after long-lasting stretch-shortening cycle exercise in humans. Eur J Appl Physiol Occup Physiol, 78, 403-10.

BAKER, B. A., MERCER, R. R., GERONILLA, K. B., KASHON, M. L., MILLER, G. R. \& CUTLIP, R. G. (2006) Stereological analysis of muscle morphology following exposure to repetitive stretch-shortening cycles in a rat model. Appl Physiol Nutr Metab, 31, 167-79.

BENZ, R. J., FRIDEN, J. \& LIEBER, R. L. (1998) Simultaneous stiffness and force measurements reveal subtle injury to rabbit soleus muscles. Molecular \& Cellular Biochemistry, $179,147-58$.

BERNARD, B. P., PUTZ-ANDERSON, V., BURT, S. E., COLE, L. L., FAIRFIELD-ESTILL, C., FINE, L. J., GRANT, K. A., GJESSING, C., JENKINS, L., HURRELL, J. J., NELSON, N., PFIRMAN, D., ROBERTS, R. L., STETSON, D., HARING-SWEENEY, M. \& TANAKA, S. (1997) Musculoskeletal disorders and workplace factors. A critical review of epidemiologic evidence for work-related musculoskeletal disorders of the neck, upper extremity, and low back. Cincinnati, NIOSH.

BOOTH, F. W., WEEDEN, S. H. \& TSENG, B. S. (1994) Effect of aging on human skeletal muscle and motor function. Med Sci Sports Exerc, 26, 556-60.

BROOKS, S. V. \& FAULKNER, J. A. (1988) Contractile properties of skeletal muscles from young, adult and aged mice. $J$ Physiol, 404, 71-82.

BROOKS, S. V. \& FAULKNER, J. A. (1990) Contraction-induced injury: recovery of skeletal muscles in young and old mice. Am J Physiol, 258, C436-42.

BROOKS, S. V. \& FAULKNER, J. A. (1994) Isometric, shortening, and lengthening contractions of muscle fiber segments from adult and old mice. Am J Physiol, 267, C507-13. 
BROOKS, S. V. \& FAULKNER, J. A. (1996) The magnitude of the initial injury induced by stretches of maximally activated muscle fibres of mice and rats increases in old age. $J$ Physiol, 497 ( Pt 2), 573-80.

BROOKS, S. V., OPITECK, J. A. \& FAULKNER, J. A. (2001) Conditioning of skeletal muscles in adult and old mice for protection from contraction-induced injury. J Gerontol A Biol Sci Med Sci, 56, B163-71.

BROOKS, S. V., ZERBA, E. \& FAULKNER, J. A. (1995) Injury to muscle fibres after single stretches of passive and maximally stimulated muscles in mice. $J$ Physiol, 488 ( Pt 2), 459-69.

CARSON, J. A., ALWAY, S. E. \& YAMAGUCHI, M. (1995) Time course of hypertrophic adaptations of the anterior latissimus dorsi muscle to stretch overload in aged Japanese quail. $J$ Gerontol A Biol Sci Med Sci, 50, B391-8.

CONBOY, I. M., CONBOY, M. J., WAGERS, A. J., GIRMA, E. R., WEISSMAN, I. L. \& RANDO, T. A. (2005) Rejuvenation of aged progenitor cells by exposure to a young systemic environment. Nature, 433, 760-4.

CUTLIP, R. G., GERONILLA, K. B., BAKER, B. A., KASHON, M. L., MILLER, G. R. \& SCHOPPER, A. W. (2004) Impact of muscle length during stretch-shortening contractions on real-time and temporal muscle performance measures in rats in vivo. J Appl Physiol, 96, 507-16.

CUTLIP, R. G., STAUBER, W. T., WILLISON, R. H., MCINTOSH, T. A. \& MEANS, K. H. (1997) Dynamometer for rat plantar flexor muscles in vivo. Medical \& Biological Engineering \& Computing, 35, 540-3.

DAVIS, J., KAUFMAN, K. R. \& LIEBER, R. L. (2003) Correlation between active and passive isometric force and intramuscular pressure in the isolated rabbit tibialis anterior muscle. $J$ Biomech, 36, 505-12.

DEGENS, H. \& ALWAY, S. E. (2003) Skeletal muscle function and hypertrophy are diminished in old age. Muscle Nerve, 27, 339-47.

DEVOR, S. T. \& FAULKNER, J. A. (1999) Regeneration of new fibers in muscles of old rats reduces contraction-induced injury. J Appl Physiol, 87, 750-6.

EDDINGER, T. J., CASSENS, R. G. \& MOSS, R. L. (1986) Mechanical and histochemical characterization of skeletal muscles from senescent rats. Am J Physiol, 251, C421-30.

ETTEMA, G. J. (1996) Mechanical efficiency and efficiency of storage and release of series elastic energy in skeletal muscle during stretch-shorten cycles. J Exp Biol, 199 ( Pt 9), 1983-97. 
EVANS, W. J. \& CAMPBELL, W. W. (1993) Sarcopenia and age-related changes in body composition and functional capacity. $J$ Nutr, 123, 465-8.

FAULKNER, J. A., JONES, D. A. \& ROUND, J. M. (1989) Injury to skeletal muscles of mice by forced lengthening during contractions. Q J Exp Physiol, 74, 661-70.

FAULKNER, J. A., OPITECK, J. A. \& BROOKS, S. V. (1992) Injury to skeletal muscle during altitude training: induction and prevention. Int J Sports Med, 13 Suppl 1, S160-2.

GERONILLA, K. B., MILLER, G. R., MOWREY, K. F., WU, J. Z., KASHON, M. L., BRUMBAUGH, K., REYNOLDS, J., HUBBS, A. \& CUTLIP, R. G. (2003) Dynamic force responses of skeletal muscle during stretch-shortening cycles. Eur J Appl Physiol, 90, 144-53.

GOSSELIN, L. E. (2000) Attenuation of force deficit after lengthening contractions in soleus muscle from trained rats. J Appl Physiol, 88, 1254-8.

HORITA, T., KOMI, P. V., NICOL, C. \& KYROLAINEN, H. (1999) Effect of exhausting stretch-shortening cycle exercise on the time course of mechanical behaviour in the drop jump: possible role of muscle damage. Eur J Appl Physiol Occup Physiol, 79, 160-7.

INGALLS, C. P., WARREN, G. L., LOWE, D. A., BOORSTEIN, D. B. \& ARMSTRONG, R. B. (1996) Differential effects of anesthetics on in vivo skeletal muscle contractile function in the mouse. Journal of Applied Physiology, 80, 332-40.

INGALlS, C. P., WARREN, G. L., WILliAMS, J. H., WARD, C. W. \& ARMSTRONG, R. B. (1998) E-C coupling failure in mouse EDL muscle after in vivo eccentric contractions. J Appl Physiol, 85, 58-67.

KLITGAARD, H., BRUNET, A., MATON, B., LAMAZIERE, C., LESTY, C. \& MONOD, H. (1989a) Morphological and biochemical changes in old rat muscles: effect of increased use. $J$ Appl Physiol, 67, 1409-17.

KLITGAARD, H., MARC, R., BRUNET, A., VANDEWALLE, H. \& MONOD, H. (1989b) Contractile properties of old rat muscles: effect of increased use. J Appl Physiol, 67, 1401-8.

KOH, T. J., PETERSON, J. M., PIZZA, F. X. \& BROOKS, S. V. (2003) Passive stretches protect skeletal muscle of adult and old mice from lengthening contraction-induced injury. $J$ Gerontol A Biol Sci Med Sci, 58, 592-7.

KOMI, P. V. (2000) Stretch-shortening cycle: a powerful model to study normal and fatigued muscle. J Biomech, 33, 1197-206. 
LIEBER, R. L. \& FRIDEN, J. (1993) Muscle damage is not a function of muscle force but active muscle strain. J Appl Physiol, 74, 520-6.

LOWE, D. A. \& ALWAY, S. E. (1999) Stretch-induced myogenin, MyoD, and MRF4 expression and acute hypertrophy in quail slow-tonic muscle are not dependent upon satellite cell proliferation. Cell Tissue Res, 296, 531-9.

LOWE, D. A., LUND, T. \& ALWAY, S. E. (1998) Hypertrophy-stimulated myogenic regulatory factor mRNA increases are attenuated in fast muscle of aged quails. Am J Physiol, 275, C155-62.

LYNCH, G. S. \& FAULKNER, J. A. (1998) Contraction-induced injury to single muscle fibers: velocity of stretch does not influence the force deficit. Am J Physiol, 275, C1548-54.

MACPHERSON, P. C., SCHORK, M. A. \& FAULKNER, J. A. (1996) Contraction-induced injury to single fiber segments from fast and slow muscles of rats by single stretches. Am J Physiol, 271, C1438-46.

MANFREDI, T. G., FIELDING, R. A., O'REILLY, K. P., MEREDITH, C. N., LEE, H. Y. \& EVANS, W. J. (1991) Plasma creatine kinase activity and exercise-induced muscle damage in older men. Med Sci Sports Exerc, 23, 1028-34.

MCBRIDE, T. A., GORIN, F. A. \& CARLSEN, R. C. (1995) Prolonged recovery and reduced adaptation in aged rat muscle following eccentric exercise. Mech Ageing Dev, 83, 185-200.

SACCO, P. \& JONES, D. A. (1992) The protective effect of damaging eccentric exercise against repeated bouts of exercise in the mouse tibialis anterior muscle. Exp Physiol, 77, 757-60.

STEVENS, E. D. (1996) Effect of phase of stimulation on acute damage caused by eccentric contractions in mouse soleus muscle. J Appl Physiol, 80, 1958-62.

STEVENS, E. D. \& FAULKNER, J. A. (2000) The capacity of mdx mouse diaphragm muscle to do oscillatory work. J Physiol, 522 Pt 3, 457-66.

UNDERWOOD, E. E. (1970) Quantatative Stereology, Reading, MA, Addison-Wesley Publishing Co.

VAN DER MEULEN, J. H., MCARDLE, A., JACKSON, M. J. \& FAULKNER, J. A. (1997) Contraction-induced injury to the extensor digitorum longus muscles of rats: the role of vitamin E. J Appl Physiol, 83, 817-23.

WARREN, G. L., 3RD, WILLIAMS, J. H., WARD, C. W., MATOBA, H., INGALLS, C. P., HERMANN, K. M. \& ARMSTRONG, R. B. (1996) Decreased contraction economy in mouse EDL muscle injured by eccentric contractions. J Appl Physiol, 81, 2555-64. 
WEIBEL, E. R. (1972) The value of stereology in analysing structure and function of cells and organs. J Microsc, 95, 3-13.

WEIBEL, E. R. (1974) Selection of the best method in stereology. J Microsc, 100, 261-9.

WEIBEL, E. R. (1975) Quantitation in morphology: possibilities and limits. Beitr Pathol, 155, 117.

WILLEMS, M. E. \& STAUBER, W. T. (2001) Force deficits after repeated stretches of activated skeletal muscles in female and male rats. Acta Physiol Scand, 172, 63-7.

WONG, T. S. \& BOOTH, F. W. (1990) Protein metabolism in rat tibialis anterior muscle after stimulated chronic eccentric exercise. J Appl Physiol, 69, 1718-24.

ZERBA, E. \& FAULKNER, J. A. (1990) A Single Lengthening Contraction can Induce Injury to Skeletal Muscle Fibers. Physiologist, 33, A122.

ZERBA, E., KOMOROWSKI, T. E. \& FAULKNER, J. A. (1990) Free radical injury to skeletal muscles of young, adult, and old mice. Am J Physiol, 258, C429-35. 


\section{Figure Legends}

Figure 1: A) Real-time force and position trace for one set of ten intermittent SSCs. The displayed force is in Newtons and angular position is in degrees. B) Real-time force and position trace for a single SSC. The displayed force is in Newtons and angular position is in degrees.

Figure 2: Pre-test isometric force of the young and old groups at each of the fourteen exposures during the chronic exposure period. The pre-test isometric force response over the chronic exposure period was quite different for the old and young age groups $(\mathrm{p}=0.0003)$. The old and young groups generated very similar magnitudes of isometric force on the first day of exposure $(p=0.455)$. The isometric forces between the two groups diverged throughout the exposure period, resulting in a substantial difference in isometric force generated by the two age groups by the end of the thirty day exposure period (fourteenth exposure, $\mathrm{p}<0.0001$ ). Data are reported as mean values $+/$ - standard error.

Figure 3: A) Peak force ( $\left.\mathrm{F}_{\text {peak }}\right)$ of the young and old groups at each of the fourteen exposures during the chronic exposure period. The peak force response over the chronic exposure period was quite different for the old and young age groups $(\mathrm{p}=0.0081)$. The old and young groups generated very similar magnitudes of peak force on the first day of exposure $(p=0.761)$. The peak forces between the two groups diverged throughout the exposure period, resulting in a substantial difference in peak force generated by the two age groups by the end of the thirty day exposure period (fourteenth exposure, $\mathrm{p}<0.0001$ ). Data are reported as mean values $+/$ - standard error. B) Minimum force $\left(\mathrm{F}_{\mathrm{min}}\right)$ of the young and old groups at each of the fourteen exposures during the chronic exposure period. The minimum force response over the chronic exposure 
period was quite different for the old and young age groups $(\mathrm{p}=0.0011)$. The old and young groups generated very similar magnitudes of minimum force on the first day of exposure $(p=$ 0.853). The minimum forces between the two groups diverged throughout the exposure period, resulting in a substantial difference in minimum force generated by the two age groups by the end of the thirty day exposure period (fourteenth exposure, $p<0.0001$ ). Data are reported as mean values $+/$ - standard error.

Figure 4: Single SSC work parameters from the single SSCs performed prior to each SSC protocol. A) The two age groups exhibited the same ability to absorb negative work during the first exposure $(\mathrm{p}=0.455)$. The ability to absorb negative work differed between age groups with subsequent exposures during the chronic exposure period $(\mathrm{p}=0.0044)$. The young group increased its negative work by an average of $27.0 \%$, while the old groups' negative work decreased by $24.9 \%$, resulting in a substantial difference by the end of the chronic exposure period $(\mathrm{p}<0.0011)$. Data are reported as mean values $+/$ - standard error. B) The two age groups exhibited the same ability to produce positive work during the first exposure $(\mathrm{p}=0.475)$. The ability to produce positive work differed between age groups with subsequent exposures during the chronic exposure period $(\mathrm{p}=0.0011)$. The young group increased its' positive work by an average of $26.5 \%$, while the old groups' positive work decreased by $39.2 \%$, resulting in a substantial difference by the end of the chronic exposure period $(\mathrm{p}<0.0011)$. Data are reported as mean values $+/$ - standard error.

Figure 5: Muscle wet weights of the left (exercised) and right (control) tibialis anterior muscle (standardized to body weight) for young and old animals after the chronic exposure. Exercised 
muscles from the young animals are significantly greater than the control muscles from the young animals and exercised and control muscles from the old animals. Control muscles from the young animals are significantly greater than both the control and exercised muscles from the old animals. The exercised muscles from the old animals are not statistically different from the control muscles in the old animals. Different letters denote statistical significance at the 0.05 level. Data are reported as mean values +/- standard error.

Figure 6: Hematoxylin and Eosin stained sections of the exercised tibialis anterior muscle (LTA) and non-exercised tibialis anterior muscle (RTA) from young rats (Figures 5a and b) and old rats (Figures 5c and d) respectively. Micrographs shown are representative of the overall groups. The micrograph from the LTA of the young animals (Figure 5a) depicts normal morphology and larger fibers than the non-exercised RTA (Figure 5b). The micrograph from the LTA of old animals (Figure 5c) depicts cellular infiltrates, and central nucleated fibers that are not evident in the samples from the non-exercised limb in either the old animals (Figure 5d) or young animals (Figures 5a and b). All micrographs are captured at 40X magnification.

Figure 7: Mean cross-sectional area $\left(\mathrm{CSA}, \mu \mathrm{m}^{2}\right)$ distribution in young and old contra-lateral, non-exercised tibialis anterior muscle (RTA). The frequency histogram depicts the frequency of fibers which lie above/below our arbitrary cut-off of $1500 \mathrm{um}^{2}$. Approximately $45 \%$ of the fibers from the older animals and $54 \%$ of the fibers from the younger animals were $\geq 1500 \mathrm{um}^{2}$.

Figure 8: Mean cross-sectional area $\left(\mathrm{CSA}, \mu \mathrm{m}^{2}\right)$ distribution in young and old exercised tibialis anterior muscle (LTA). The frequency histogram depicts the frequency of fibers which lie 
above/below our arbitrary cut-off of $1500 \mathrm{um}^{2}$. Approximately $46 \%$ of the fibers from the older animals and $76 \%$ of the fibers from the younger animals were $\geq 1500 \mathrm{um}^{2}$. Note the occurrence of small fibers $\left(\leq 1500 \mathrm{um}^{2}\right)$ in the old, exercised tibialis anterior muscle.

Figure 9: Isometric force normalized to muscle wet weight of the tibialis anterior after the chronic exposure period (N/mg). The young group exhibited values that are significantly greater than the old group. Significance is reported at the 0.05 level. Data are reported as mean values +/- standard error.

Figure 10: Volume density (\% volume fraction) of the cellular interstium (CI) in the young and old animals' left (treated) and right (control) tibialis anterior. The volume density of the CI was greater in the treated limb of the old animals than all other groups. All other comparisons were not significantly different. Different letters denote significance at the 0.05 level. Data are reported as mean values $+/$ - standard error. 


\section{Figure 1}

Typical Force and Angular Position Graph for Intermittent SSCs

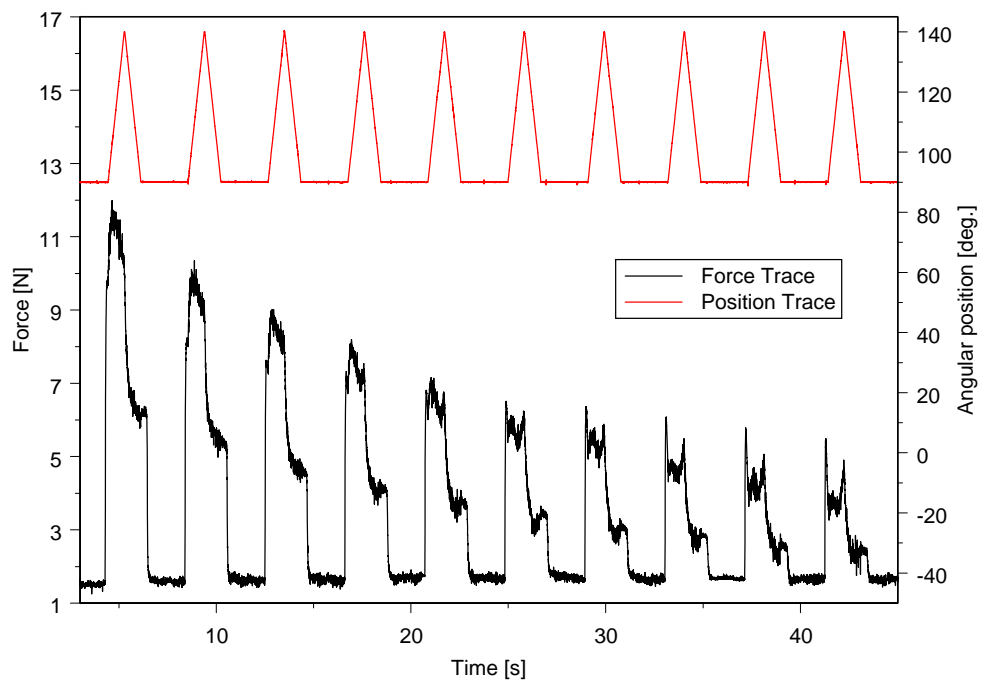

(a)

Typical Force and Angular Position Graph for Single SSC

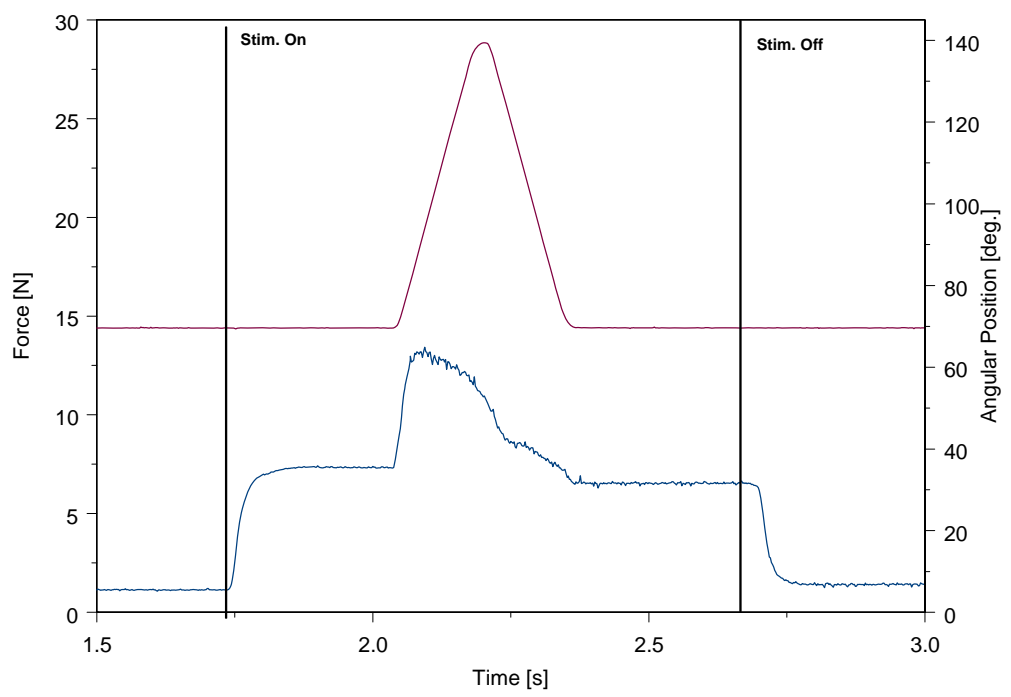

(b) 
Figure 2

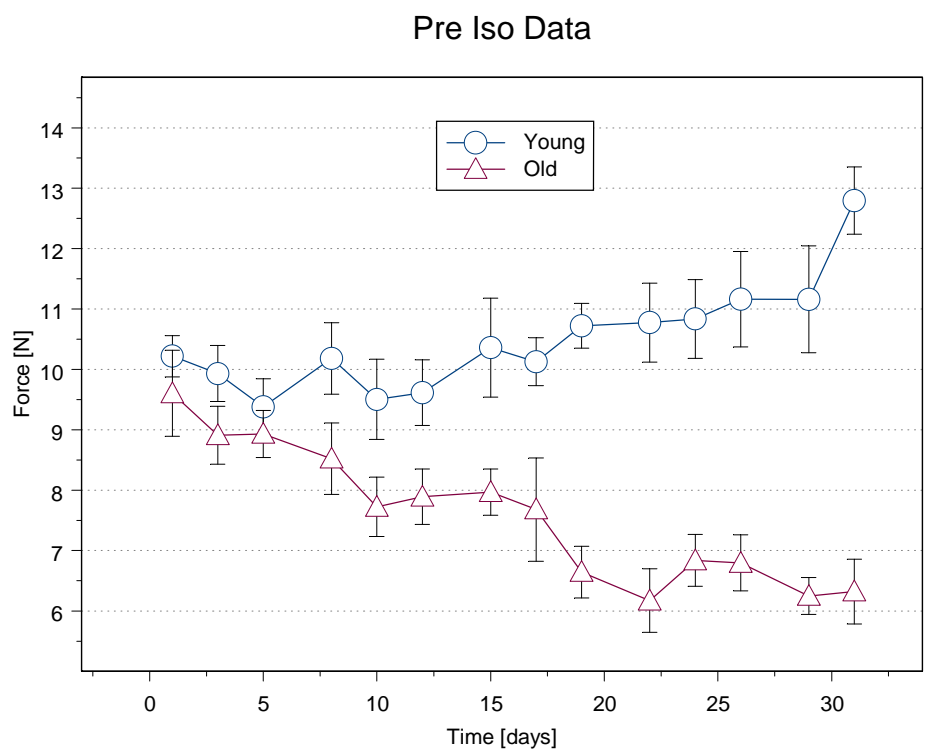


Figure 3

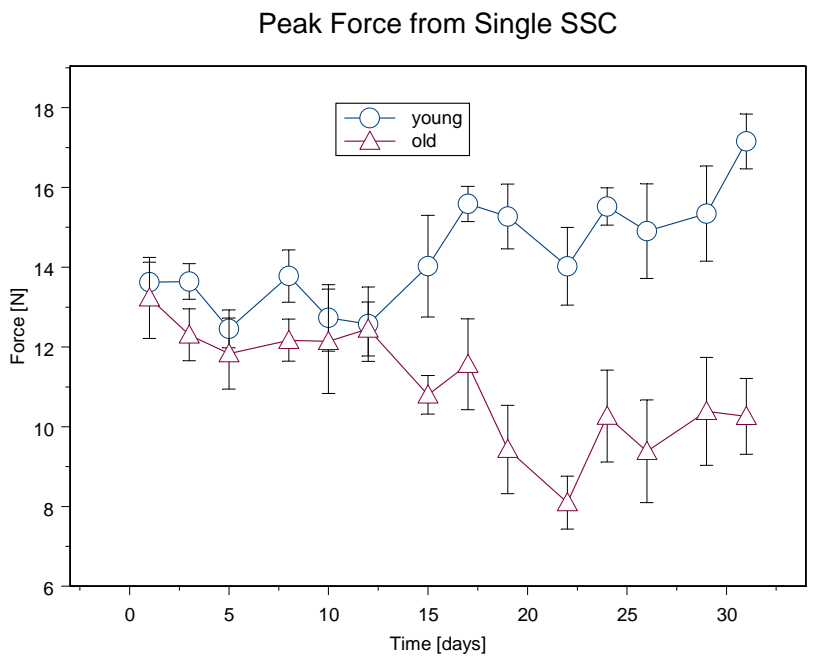

(a)

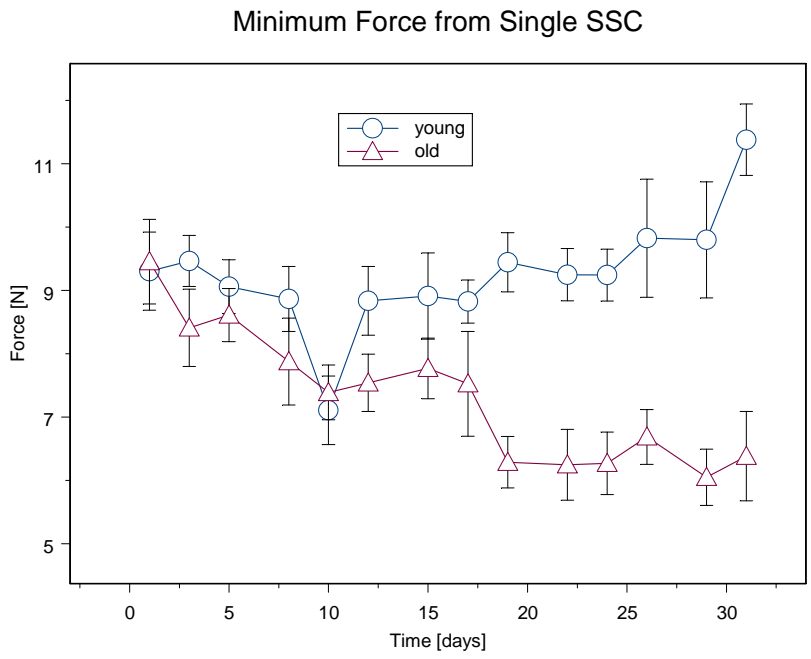

(b) 


\section{Figure 4}

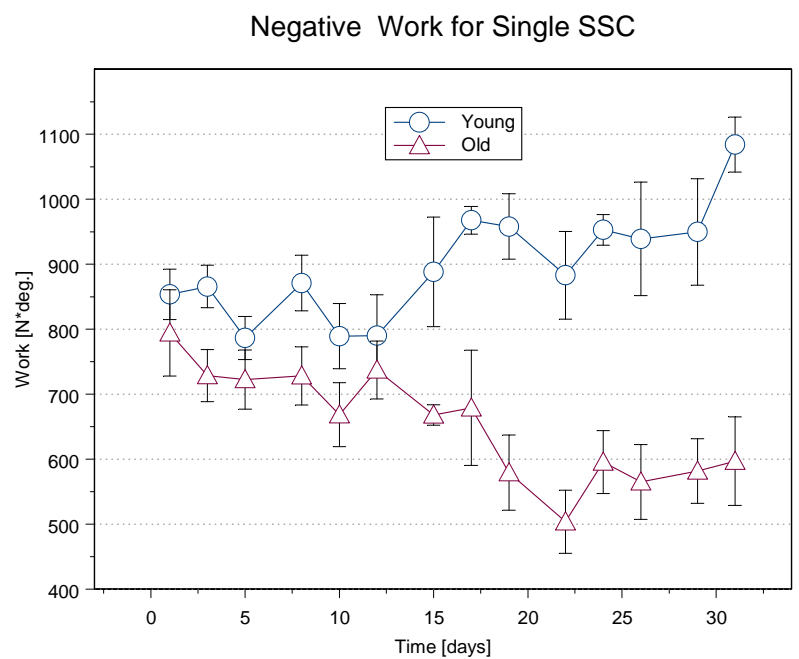

(a)

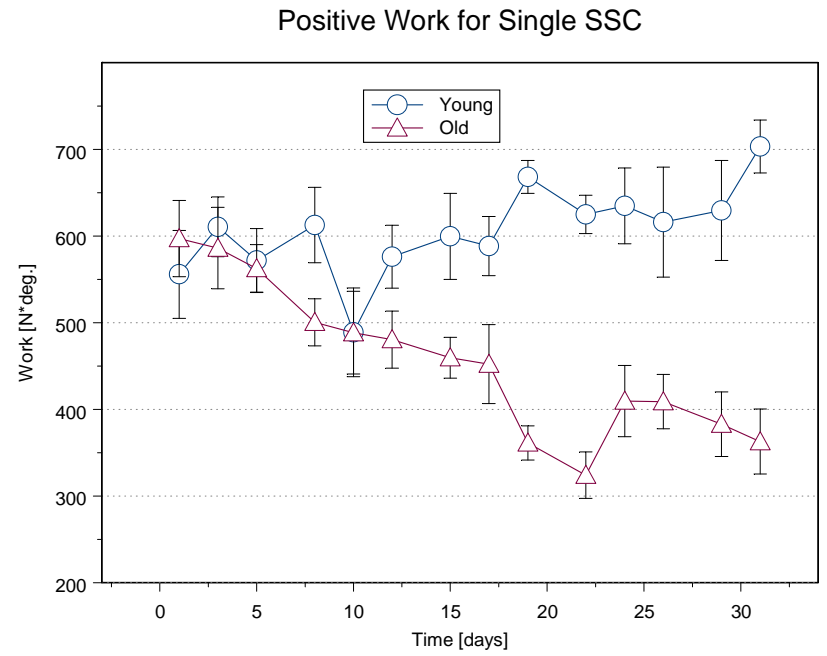

(b) 
Figure 5

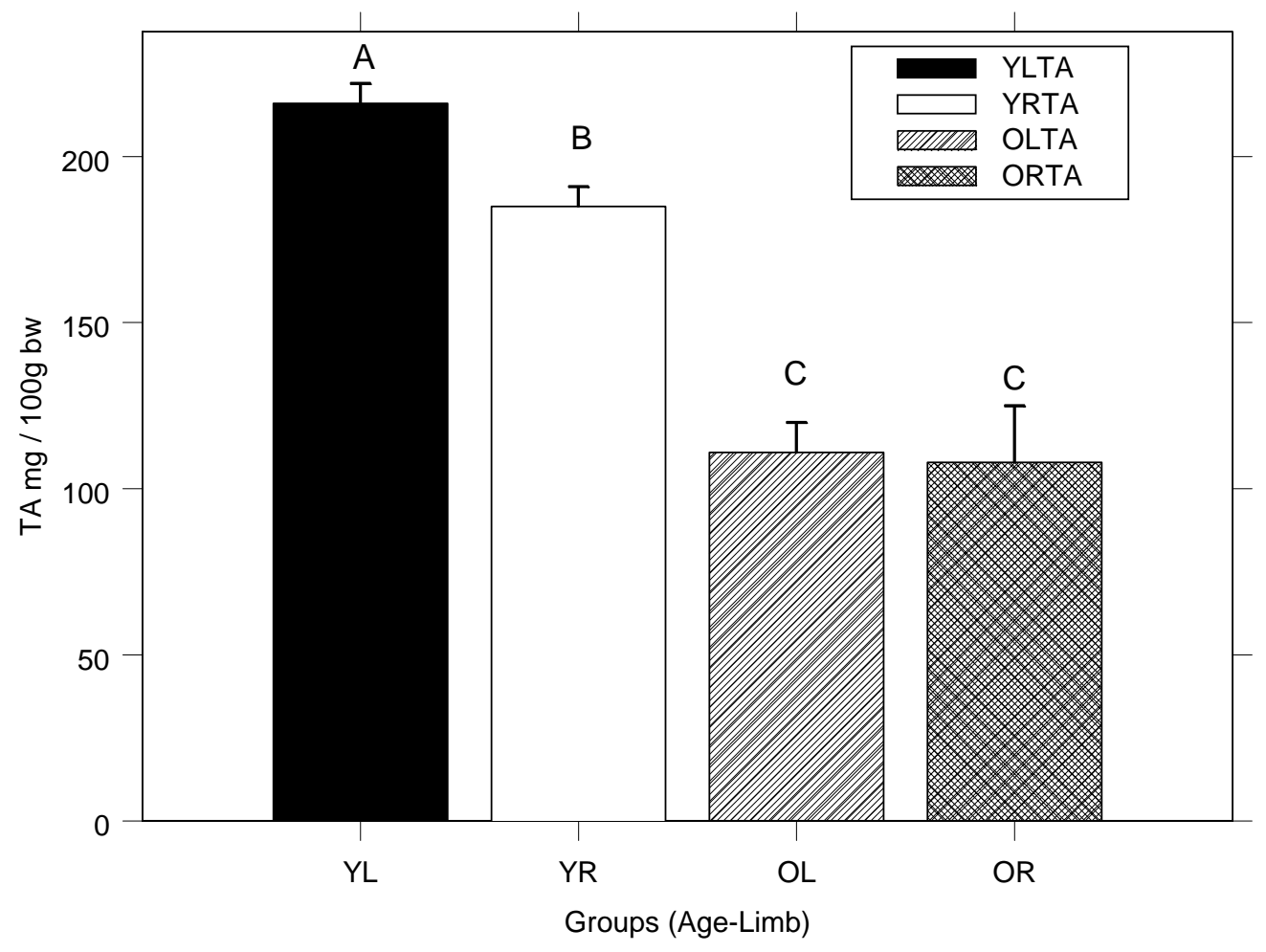


Figure 6

Young LTA

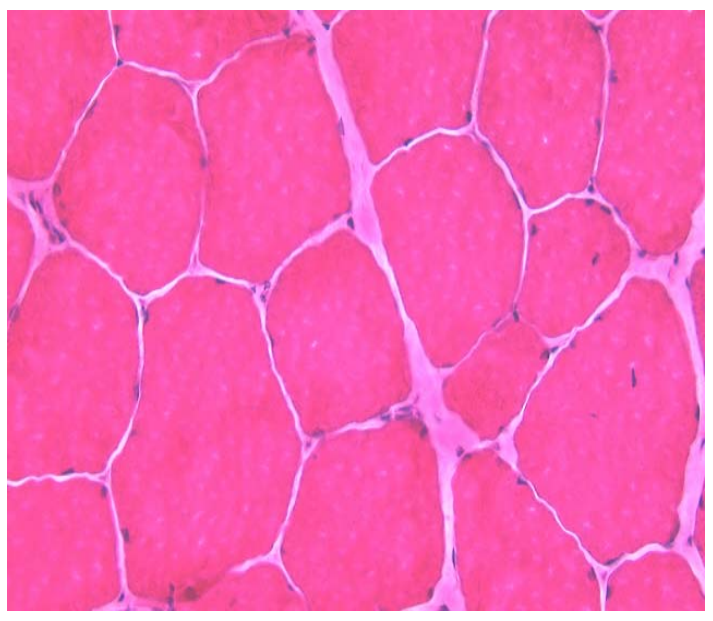

(a)

Old LTA

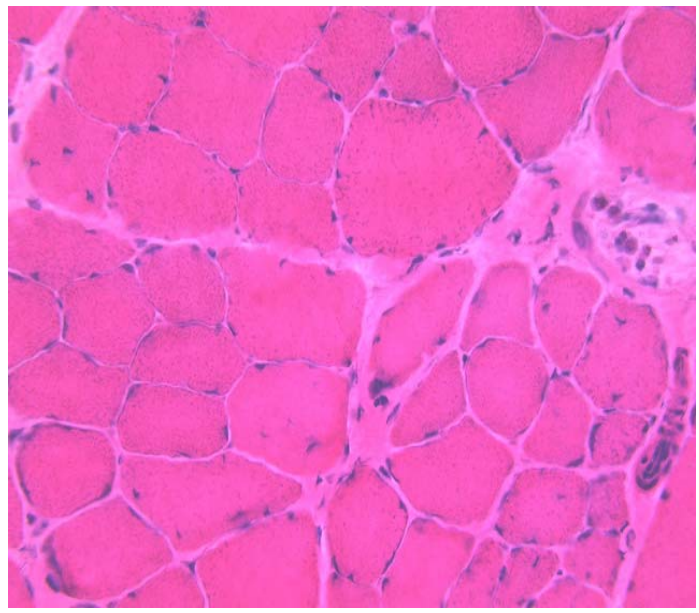

(c)
Young RTA

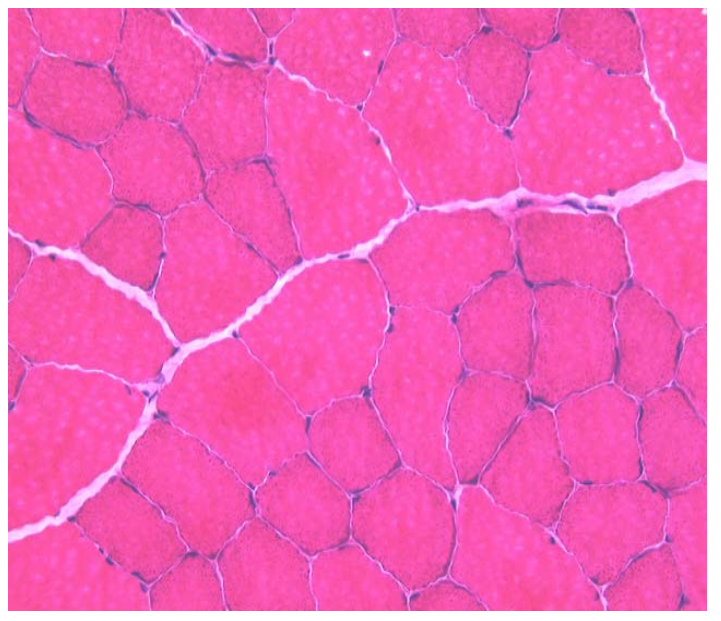

(b)

\section{Old RTA}

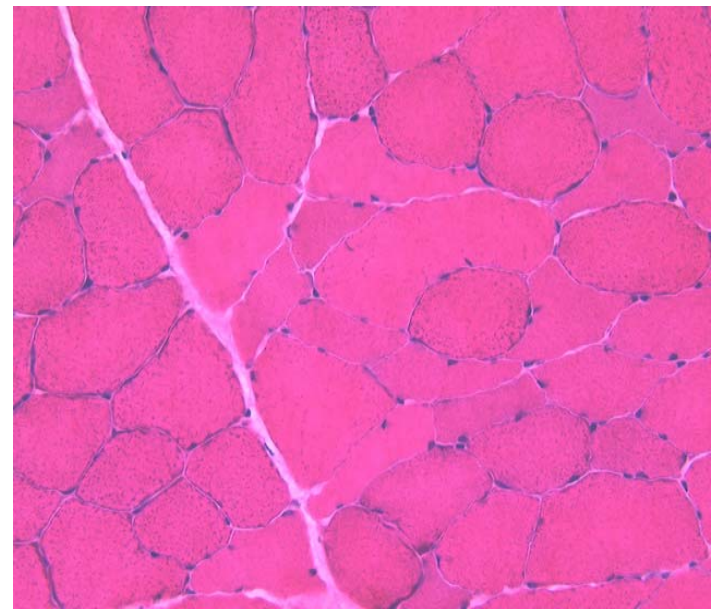

(d) 
Figure 7

Right

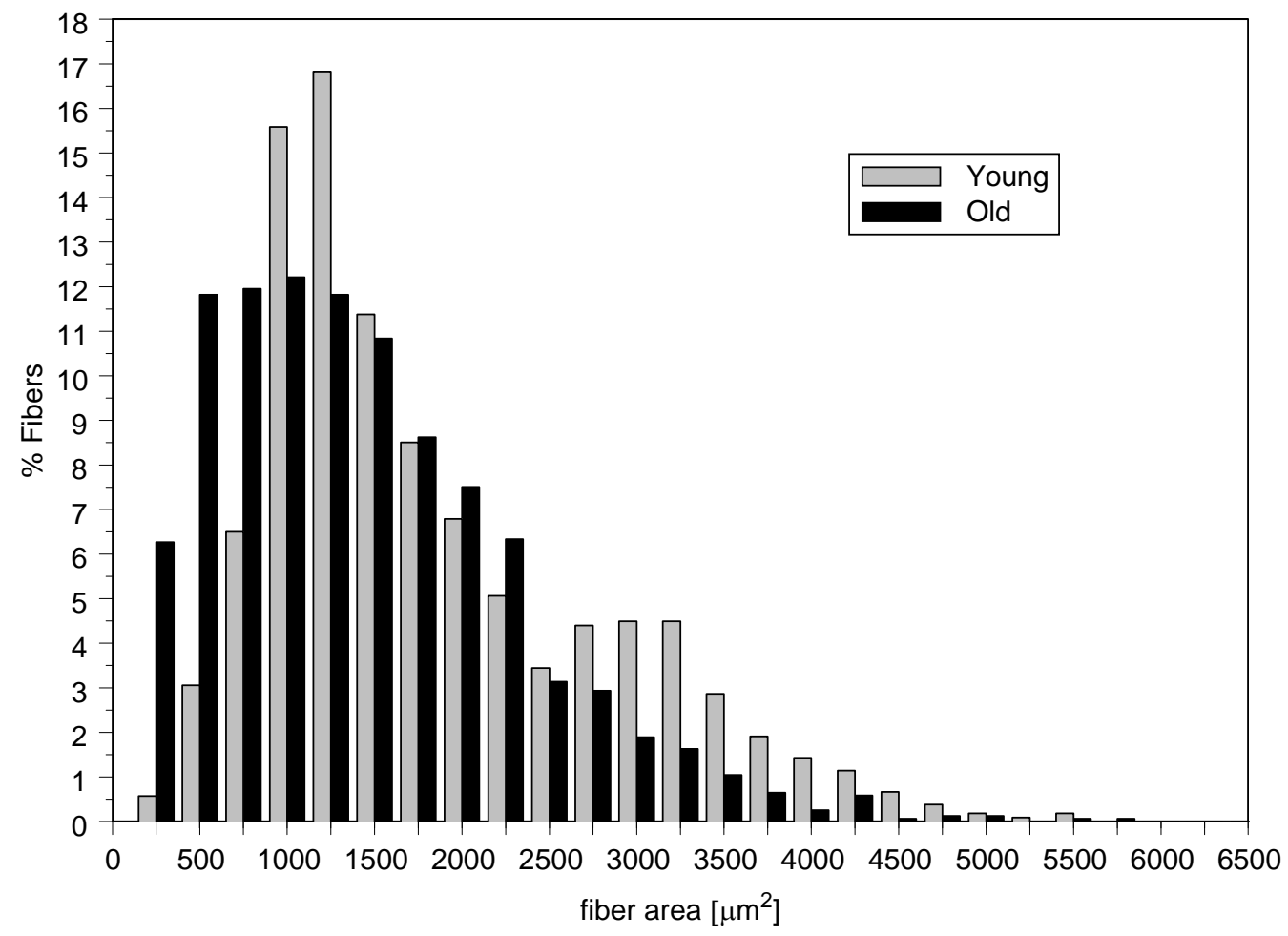


Figure 8

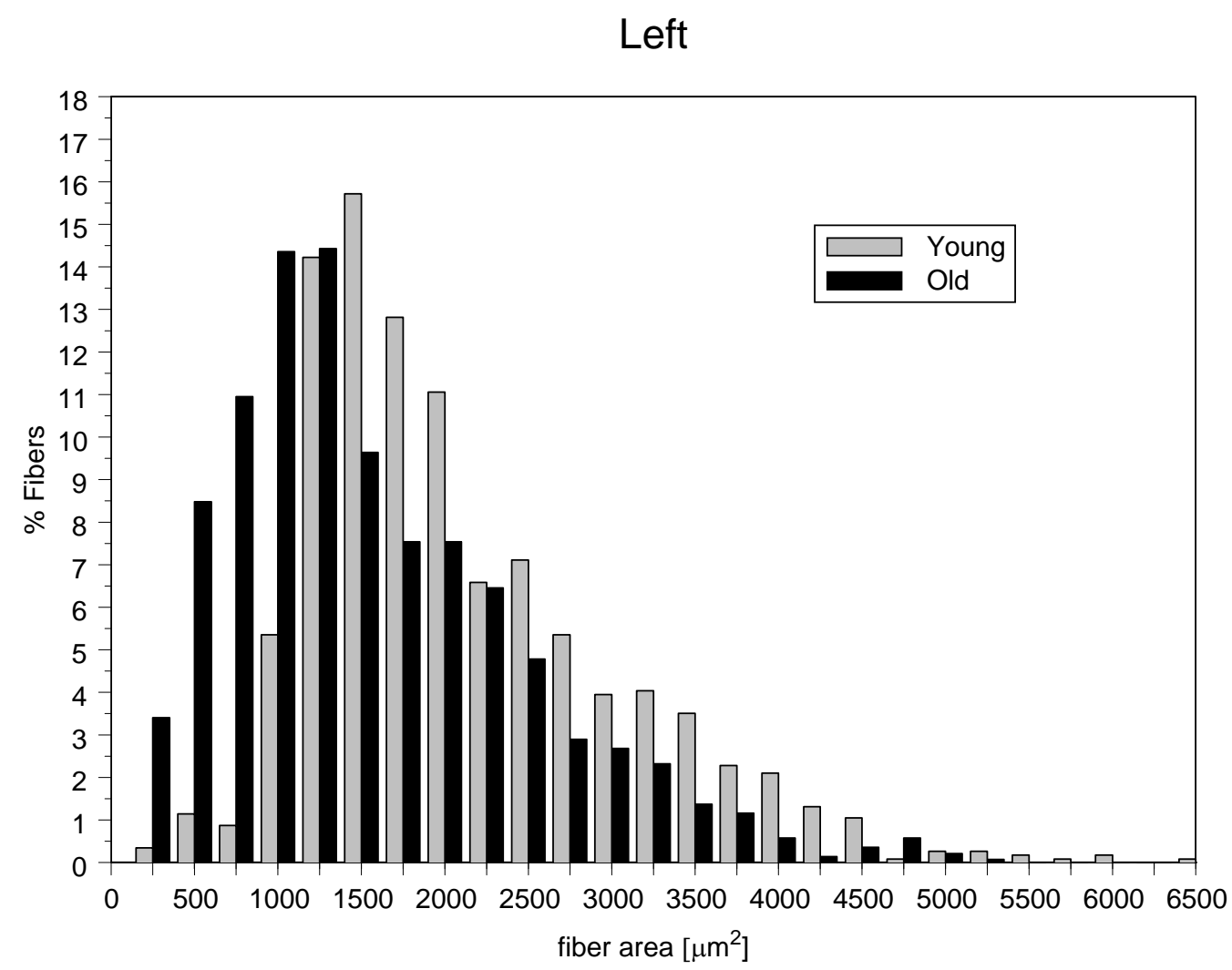


Figure 9

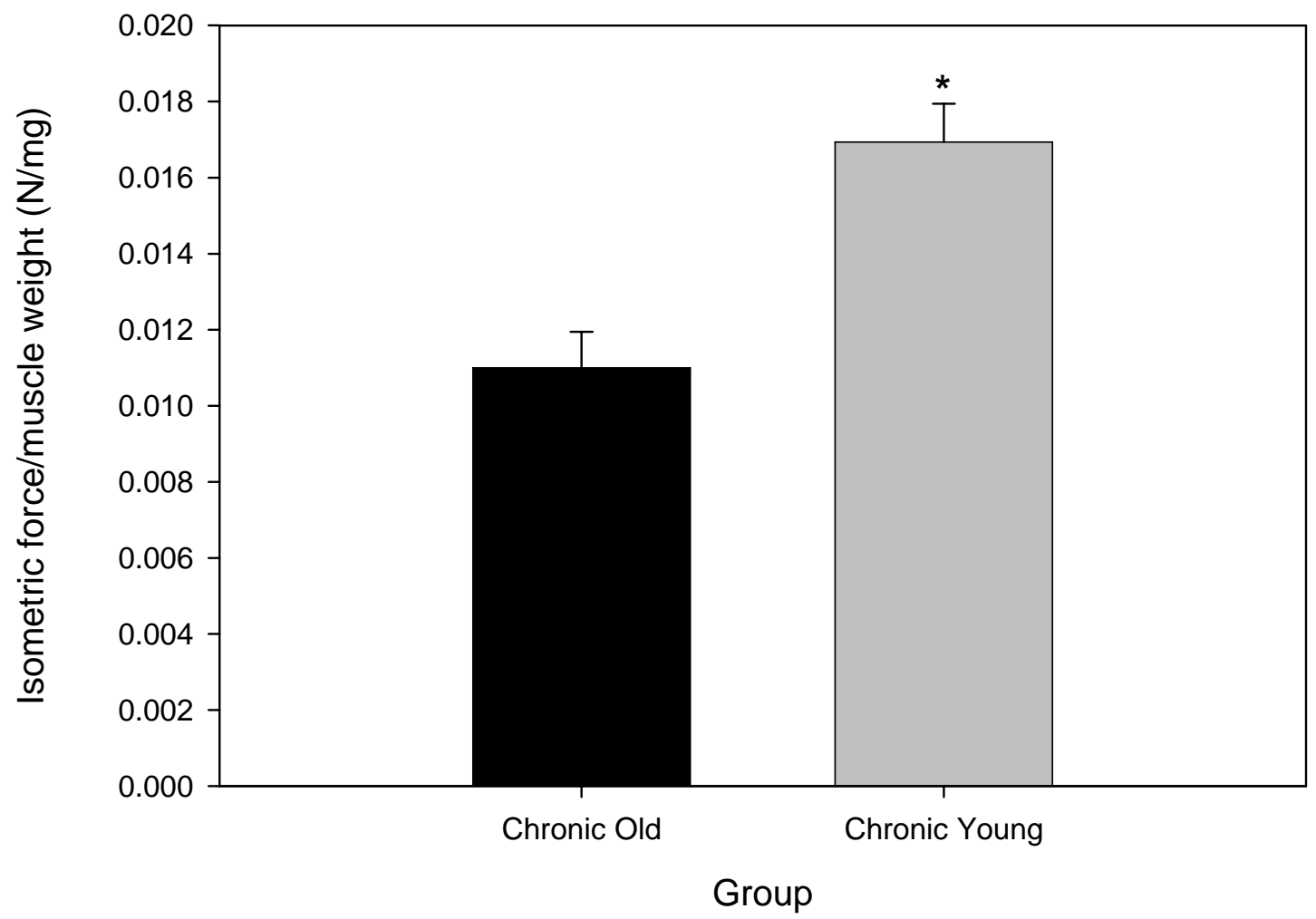


Figure 10

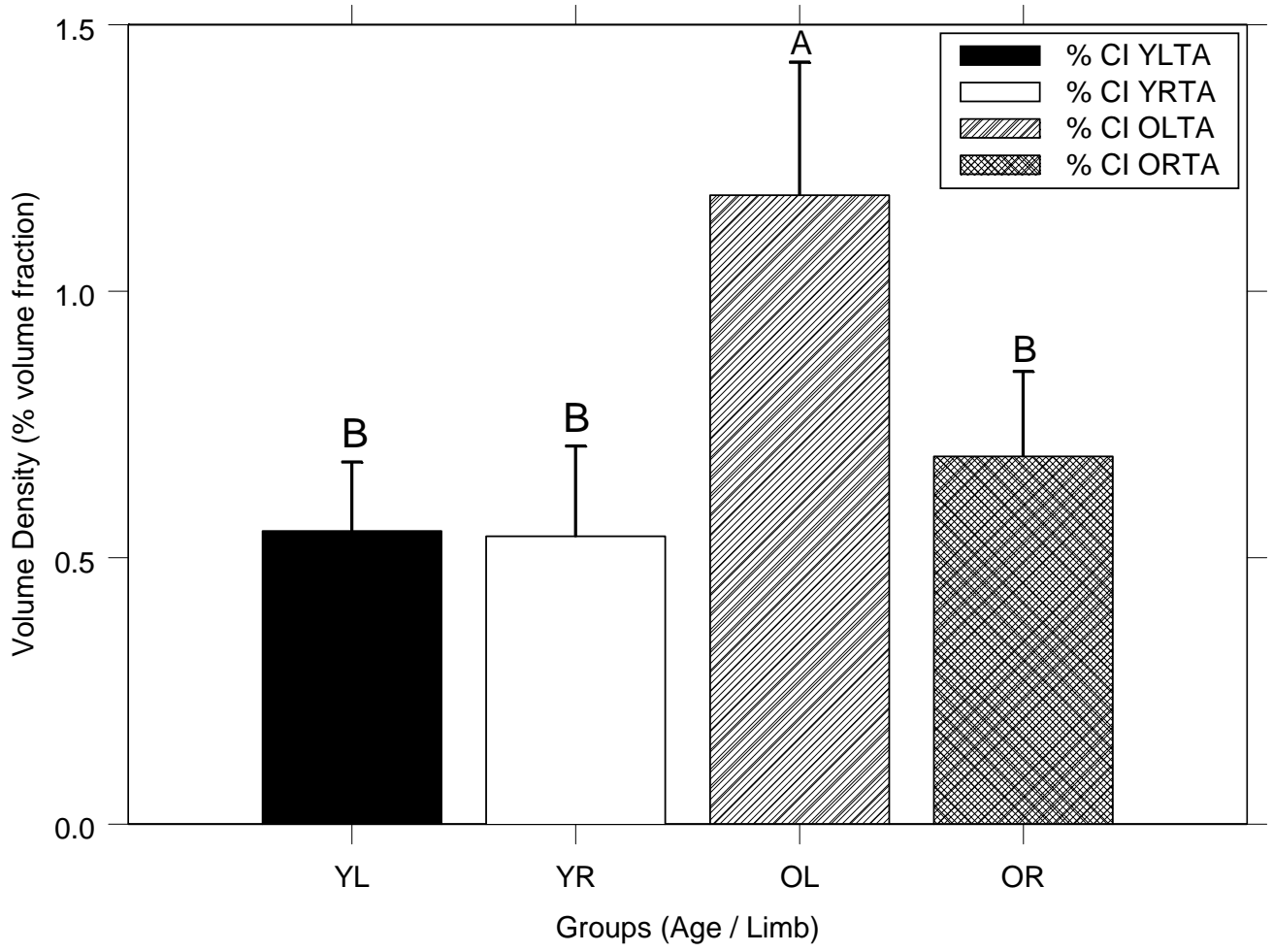


Effects of Glutathione Depletion and Age on Performance and Skeletal Muscle Morphology Following Chronic Stretch-Shortening Contraction Exposure

\section{Brent A. Baker ${ }^{1}$, Melinda S. Hollander ${ }^{1}$, Michael L. Kashon ${ }^{1}$, Robert G. Cutlip $^{1}$}

${ }^{1}$ National Institute for Occupational Safety and Health (NIOSH), Health Effects Laboratory Division, Morgantown, WV, 26506, USA.

Address correspondence to:

Robert G. Cutlip, Ph.D.

National Institute for Occupational Safety and Health

Health Effects Laboratory Division

1095 Don Nehlen Drive

$\mathrm{M} / \mathrm{S} 2027$

Morgantown, WV 26505

Fax: (304) 285-6265; Tel: (304) 285-5968; E-mail: rgc8@cdc.gov

Running head: Glutathione Depletion and Chronic Stretch-Shortening Contraction Exposure 


\section{ABSTRACT}

Background: The involvement of glutathione in the adaptive response of skeletal muscle following repetitive, high-intensity mechanical loading is not known. Aim: The purpose of this research was to examine the influence dietary supplementation of a glutathione antagonist (LButhionine Sulfoximine (BSO)) has on the adaptability of skeletal muscle during chronic highintensity mechanical loading via stretch-shortening contractions in young and old rodents.

Research design and methods: Left dorsiflexor muscles of young (12 weeks, $N=16)$ and old (30 months, $\mathrm{N}=16$ ), vehicle- and BSO-treated rodents were exposed 3 times per week for 4.5weeks to a protocol of 80 maximal SSCs per exposure in vivo. Skeletal muscle response was characterized by static and dynamic performance, as well as muscle wet-weight and quantitative morphological analyses following the exposure period. Results: BSO treatment had little deleterious effect on muscle performance and morphology following chronic SSC exposures. Generally, muscle performance increased in the young rodents following the chronic exposure to SSC, while no significant change was noted in the old rodents. Muscle-wet weight was increased following loading of the exposed limb compared with the contra-lateral control limb, irrespective of age. Further, young rodents had significantly increased muscle wet-weight compared to old. No degenerative myofibers were noted in either age group, but a significant increase was observed in the volume of non-cellular interstitium as a result of aging $(\mathrm{p}<0.05)$, which is indicative of edema. Conclusions: Even though adaptive changes are observed with old rodents, aging negatively influences the adaptive response to chronic SSC exposure by limiting performance as well as physiological adaptation; however glutathione depletion does not adversely effect nor exacerbate this adaptive profile.

Keywords: Aging, Glutathione, Stretch-Shortening Contractions, Muscle Hypertrophy, Inflammation

\section{Publications Disclaimers:}

"The findings and conclusions in this report are those of the author(s) and do not necessarily represent the views of the National Institute for Occupational Safety and Health." 


\section{INTRODUCTION}

\section{Paragraph 1}

High-intensity mechanical loading (as encountered during occupational exposure or resistance training) is comprised of high relative force production due to increased intensity/load and may be influenced by duty cycle, velocity of movement, muscle length, and number of repetitions (Cutlip et al., 2005, Cutlip et al., 2004, Warren et al., 1991, Warren et al., 1993a, Stauber, 2004, Baker et al., In press-a). Following mechanical loading of skeletal muscle, we and others have shown this to result in skeletal muscle injury (Baker et al., 2006b, Clarkson and Hubal, 2002, Baker et al., 2006a, Geronilla et al., 2003). However, overt skeletal muscle injury does not always occur following mechanical loading in naive rodents (Cutlip et al., 2006) (Baker et al., In review 2007). Recently, we have demonstrated that chronic exposure of rodents to stretchshortening contractions (SSCs) does not produce skeletal muscle degeneration, regardless of age, and aging may influence the adaptive capacity to training via unresolved, low-grade inflammation (Cutlip et al., 2006). Imperative in the understanding of adaptation resulting from mechanical exposure is that not all perturbations result in skeletal muscle degeneration, but may be influenced across an adaptive continuum by the inflammatory response or possibly changes due to oxidative stress mismanagement. Consistent with the literature is a recent study from our lab, which has shown that depletion of total glutathione levels in the target muscle can be achieved using L-Buthionine Sulfoximine (BSO), a glutathione antagonist, irrespective of age (Hollander et al., In preparation 2007). Additionally, BSO treatment increased measures of hydrogen peroxide in both young and old rodents (indicating increased oxidative stress), yet this increase in hydrogen peroxide was prevented with chronic, repetitive SSC exposure in young rodents only. Also chronic SSC exposure increased indices of lipid peroxidation and cell death 
in aged rodents only, but had no effect on hydrogen peroxide, irrespective of age. These findings suggest that oxidative stress and apoptosis may contribute to the diminished adaptation following mechanical loading observed with aging. Interestingly, apoptosis of satellite cells has been recently implicated in limiting the recovery following injurious SSC exposure in aged rodents (Krajnak et al., 2006).

\section{Paragraph 2}

As mentioned above, oxidative stress production following high-intensity mechanical loading has been suggested to lead to negative changes in force production (McArdle et al., 2001) and cellular responses (McBride, 1998) especially with aged skeletal muscle. It has also been suggested that reactive oxygen species (ROS) may be a mechanism contributing to the injurious response observed in skeletal muscle following resistance training/mechanical loading (Uchiyama et al., 2006). Given that oxidative stress (or the inherent balance between oxidant production and antioxidant defenses) may be a critical stimulus for skeletal muscle adaptation (Urso and Clarkson, 2003) in the absence of myofiber degeneration, modulating the oxidative stress profile and quantifying the resulting adaptive response would prove beneficial in broadening our understanding with respect to mechanical-loading induced adaptations.

\section{Paragraph 3}

Glutathione is the most important non-protein thiol source that maintains most cells in a reduced environment (Meister, 1991). Further, glutathione is a major antioxidant in the cell and serves various functions such as directly acting as a reducing agent, serving as a substrate for glutathione peroxidase (and others), and recycling radicals generated from other antioxidant 
molecules. Since skeletal muscle represents the largest pool of total glutathione in the body (due to the vast percentage of muscle comprising the body), it seems plausible that modulation of the glutathione concentration would provide insight into the adaptive capacity of skeletal muscle that may be under pro-oxidant/antioxidant regulation. As mentioned previously, glutathione depletion can be accomplished using BSO, which is an irreversible inhibitor of $\gamma$ glutamylcysteine synthase (GCS) (Meister, 1991). BSO has been well documented both physiologically and biochemically to chronically deplete levels of glutathione, and these animals would be expected to be more vulnerable to mechanical loading-induced oxidative stress (Martensson, 1989, Leeuwenburgh and Ji, 1995) (Hollander et al., In preparation 2007).

\section{Paragraph 4}

The purpose of this research was to investigate the influence dietary supplementation of a glutathione antagonist has on the adaptability of skeletal muscle during high-intensity mechanical loading (via stretch-shortening contractions (SSCs)), and to determine if aging inherently opposes the adaptive response observed in young rodents (Cutlip et al., 2006). Skeletal muscle adaptation was assessed by monitoring the changes in isometric and dynamic performance during the chronic exposure period, as well as by morphological changes in skeletal muscle after completion of the SSC protocol. Adaptation was defined by a maintenance or increase in contractile performance as a result of the repetitive exposures and the absence of morphological evidence of degeneration or inflammation. Mal-adaptation was defined as a decrease in contractile performance and the presence of morphological evidence of degeneration or inflammation as a result of the exposures. Our general hypothesis is that muscles depleted of glutathione will have a lower tolerance to chronic mechanical loading than muscles from those 
that are not glutathione depleted and present with mal-adaptation, and aging will exacerbate this mal-adaptation. Specifically, we hypothesized that muscles from young BSO rodents will not adapt to chronic mechanical loading exposures, while young rodents receiving no BSO treatment will adapt. Similarly, old BSO rodents will not adapt to the same chronic loading protocol, and old rodents will exhibit greater indices of mal-adaptation than younger counterparts supplemented with BSO. Old rodents receiving no BSO will not adapt to the same repetitive loading protocol due to a lower safety threshold or tolerance.

\section{METHODS}

\section{Paragraph 5}

Animal handling: Male Fischer Brown Norway Hybrid rats (F344 x BN F1, N = 32) were obtained from the National Institutes on Aging colony. Young adult $(\mathrm{N}=16 ; 12$ weeks) and old $(\mathrm{N}=16 ; 30$ months) rats were housed in an AAALAC accredited animal quarters. After one week of acclimatization young and old rats were randomly divided into L-Buthionine Sulfoximine (BSO-treated, $(n=6))$, regular drinking water (vehicle, VEH-treated $(n=6))$, or cage-control groups (Cage-CON $(\mathrm{n}=4))$, and all animals were subjected to a standardized experimental protocol approved by the NIOSH Animal Care and Use Committee. Temperature and light/dark cycle (dark cycle from 7:00 AM to 7:00 PM) were held constant for all animals; food and water were provided ad libitum for all groups, while water consumption was monitored for the experimental groups.

\section{Paragraph 6}


Glutathione Depletion: Rats randomized to the BSO-treated group received $300 \mathrm{ml}$ of water weekly supplemented with $10 \mathrm{mM}$ BSO in their drinking water, and water consumption was monitored weekly for all experimental groups. No differences were noted in water consumption during and after the exposure period with respect to age or treatment groups. The supplemented dose of BSO used in the current study was determined from a dose-response study in which total glutathione was significantly depleted in the tibialis anterior (TA) at the $10 \mathrm{mM}$ BSO concentration to approximately $65 \%$ of control levels (unpublished data).

\section{Paragraph 7}

Experimental setup: The dorsiflexor muscles were tested on a custom-built rodent dynamometer (Cutlip et al., 1997a). The experimental setup has been described in detail previously (Cutlip et al., 2004). Briefly, the dynamometer provides precise control over the muscle length and muscle force output parameters to be studied. The software acquired and stored position, force, and velocity data in real-time as described elsewhere (Cutlip et al., 2004). Rats were anesthetized with $2 \%$ isoflurane gas using a small animal anesthetic system (Surgivet Anesco Inc., Waukesha, WI, USA). Isoflurane was chosen because it has no effect on in vivo force production (Ingalls et al., 1996). After anesthesia, each rat was placed supine on the heated $\mathrm{x}-\mathrm{y}$ positioning table of the rodent dynamometer, with an anesthetic mask placed over its nose and mouth. The knee was secured in flexion (at $90^{\circ}$ ) with a knee holder. The left foot was secured in the load cell fixture using a custom-built foot holder with the ankle axis (assumed to be between the medial and lateral malleoli) aligned with the axis of rotation of the load cell fixture. Each animal was monitored during the protocol to ensure proper anesthetic depth and body temperature. 


\section{Paragraph 8}

Functional testing: As with the experimental set-up, the functional testing has been described in detail previously (Cutlip et al., 2004). Briefly, the joint position of the animal was defined by the angle between the tibia and the plantar surface of the foot. The angular position of the load cell fixture corresponded with the angular position of the ankle. The force produced by the dorsiflexor muscles was measured at the interface of the aluminum sleeve and the dorsum of the foot. Platinum stimulating electrodes (Grass Medical Instruments, Quincy MA, USA) were placed subcutaneously each exposure session to span the peroneal nerve. Activation of the electrical stimulator resulted in muscle contraction of the dorsiflexor muscle group. To reduce the effect of excitation-contraction fatigue, all electrical stimulation times were kept to a minimum with 2-minutes (min) of recovery time between stimulations (Ingalls et al., 1998).

\section{Paragraph 9}

SSC protocol: The SSC protocol implemented in the current study has been previously described in detail (Cutlip et al., 2006). Briefly, young and old experimental groups were exposed to 8 sets of 10 repetitions of SSCs with 2-min intervals between each set. Within each set, there was a 2-s rest between each stretch-shortening contraction. For each repetition, the dorsiflexor muscles were fully activated for $100 \mathrm{~ms}$ duration via the electrical stimulator, and the eccentric contraction phase was initiated with a $60 \mathrm{deg} / \mathrm{s}$ movement velocity of the load cell fixture over the prescribed range of motion of 90-140 deg ankle angle. The load cell fixture was then immediately returned in the concentric phase at $60 \mathrm{deg} / \mathrm{s}$ to the starting position of $90 \mathrm{deg}$ ankle angle. The dorsiflexor muscles were then deactivated $300 \mathrm{~ms}$ later. Total stimulation time per repetition was $2.06 \mathrm{~s}$. 


\section{Paragraph 10}

Isometric Force Test: A pre-test isometric contraction (pre-test isometric force) was measured on the dorsiflexor muscle group at an ankle angle of 90 deg using a 300 ms stimulation duration in a similar fashion as Davis et al. (Davis et al., 2003) and Willems and Stauber (Willems and Stauber, 2001). An isometric contraction was also performed immediately following the SSC protocol (post-test isometric force) that consisted of eight sets of ten stretch-shortening contractions.

\section{Paragraph 11}

Dynamic Force Test: A single stretch-shortening contraction was measured on the dorsiflexor muscle group two minutes preceding and following treatment with the SSC protocol as previously described (Cutlip et al., 2004). This test was used to evaluate the muscle's ability to generate dynamic forces and to perform work during dynamic stretch-shortening. The stretchshortening contraction was performed by activating the dorsiflexor muscles for $300 \mathrm{~ms}$ then moving the load cell fixture from 70-140 deg at an angular velocity of $500 \mathrm{deg} / \mathrm{s}$. The load cell fixture was immediately returned to $70 \mathrm{deg}$, at $500 \mathrm{deg} / \mathrm{s}$. Activation was continued for $300 \mathrm{~ms}$ after cessation of the movement.

\section{Paragraph 12}

Chronic Exposure: The SSC protocol and performance tests were administered three times per week for a total of 14 exposures over a four and a half week period.

\section{Paragraph 13}


Histology: Twenty-four hours after the final exposure, rats were weighed, anesthetized with sodium pentobarbital (ip, $10 \mathrm{mg} / 100 \mathrm{~g}$ body weight) and exsanguinated. The left (exposed LTA) and right (contra-lateral control - RTA) tibialis anterior muscles were dissected, weighed, divided into 5 equal-distant "zones" ("Zone 1" most proximal - "Zone 5" most distal), mounted onto cork board with OTC (VWR, West Chester, Pennsylvania), frozen in liquid nitrogen-cooled isopentane, and stored at $-80^{\circ} \mathrm{C}$ until sectioned for histology. Collection and preparation of samples was standardized, 3 minutes at room temperature was allowed for all procedures prior to freeze fixation in isopentane cooled in liquid nitrogen. "Zone 3" was selected to obtain the maximum tissue sample corresponding to the TA mid-belly. Transverse sections were cut at 12 micron $(\mu \mathrm{m})$ thickness, mounted on pre-coated microscope slides, air dried, and stained with hematoxylin \& eosin using Harris' procedure. Muscle morphology was assessed using standard stereological methods as used previously and described below.

\section{Paragraph 14}

Muscle quality: Pre-test isometric force measured at the last session of the chronic exposure period was normalized to muscle wet weight of the tibialis anterior of the exposed limb.

\section{Paragraph 15}

Fiber cross-sectional area: For muscle fiber cross-sectional area (CSA) analysis, ten nonoverlapping digital images were obtained from hematoxylin and eosin-stained muscle sections at a 40X magnification and were analyzed for fiber CSA $(\mu \mathrm{m} 2)$ with Optimas Image Analysis Software. Each fiber was traced with a handheld mouse, and the number of pixels traced was 
calibrated to a defined area in square micrometers. Approximately 200 fibers were traced per sample as previously described (Cutlip et al., 2006).

\section{Paragraph 16}

Stereology: Quantitative morphometric methods were used to measure the volume fraction, surface densities, and average thickness of normal myofibers, degenerative myofibers, and the interstitial space (Baker et al., 2006a). The interstitium was divided into endomysium and perimysium spaces, which included capillaries. A standardized stereological technique, as previously discussed (Baker et al., 2006a), was used to quantify the degree of myofiber degeneration and inflammation, which was quantified as either non-cellular interstitium (NCI), indicative of edema, or cellular interstitium (CI), indicative of cellular infiltrates. Fiber volume and surface density were measured using standard morphometric analyses (Weibel, 1972, Weibel, 1974, Weibel, 1975, Underwood, 1970). Briefly, one of the H\&E stained sections was taken from each animal. A stage micrometer was used to identify the mid-point of the section. Point and intercept counts using a 121-point / 11-line overlay graticule (12.5 mm square with 100 divisions) at 40X magnification were taken at 5 equally spaced points across the section. This process was repeated $2 \mathrm{~mm}$ on either side of the mid-point of the section for a total of 1210 points and 110 intercept lines per section. Volume density or percent volume was computed from the percentage of points over the tissue section to points over normal myofibers, degenerative myofibers, cellular interstitium and non-cellular interstitium plus capillaries (Weibel, 1972, Weibel, 1974, Weibel, 1975). Intercepts over the line overlay were counted for the perimeter of normal myofibers, degenerative myofibers, and interstitium to myofiber transitions. Points and intercepts over blood vessels greater than 25 microns $(\mu \mathrm{m})$ in diameter 
were excluded. One section per animal per group was evaluated and the results expressed as mean \pm S.E.M. Stereology was used to quantify the degree of myofiber degeneration, and the accompanying changes in the TA muscle from each group. Myofibers were defined by the following criteria: Normal myofibers demonstrated: 1) Complete contact with adjacent myofibers, 2) A smooth outer membrane, and 3) No presence of internal inflammatory cells. Degenerative myofibers displayed: 1) A loss of contact with adjacent myofibers, 2) Presence of internal inflammatory cells, and 3) An outer membrane interdigitated with inflammatory cells.

\section{DATA ANALYSIS}

\section{Paragraph 17}

Dynamic Force Test: The experimental data from exposure 1 and exposure 2 was averaged as well as data from exposure 13 and exposure 14 from the SSCs and was used to calculate the group means and standard error of the mean for isometric force, $F_{\text {peak }}$ (peak eccentric force), $F_{\min }$ (isometric pre-stretch force), negative work, and positive work. Work was calculated as previously reported by Cutlip and colleagues (Cutlip et al., 2006), as was the dynamic force parameters $\mathrm{F}_{\text {peak }}$ and $\mathrm{F}_{\min }$. Isometric force, dynamic forces, and work measures were analyzed using a two-way analyses of variance with repeated measures (treatment x age). All data are presented as means \pm SEM unless otherwise noted, and differences with a probability of $\mathrm{p}<0.05$ were considered significant.

\section{Paragraph 18}

Muscle wet-weights: Data for muscle wet-weight was normalized to tibia length and differences between the groups were analyzed using a three-way (treatment $\mathrm{x}$ age $\mathrm{x}$ limb) ANOVA with 
animal as the random factor accounting for measures in both limbs. Differences in muscle wetweights between the exposed limb for each group following exposure and the corresponding contra-lateral control muscle were analyzed using an unpaired $t$ test.

\section{Paragraph 19}

Stereology: Stereological measurements for volume of cellular and non-cellular components and muscle quality were analyzed using three-way mixed model (treatment $\mathrm{x}$ age $\mathrm{x}$ limb) ANOVA with the animal as the random factor accounting for measures in both limbs. All data were analyzed using JMP v. 5.1 (SAS Institute Inc., Cary, NC).

\section{$\underline{\text { RESULTS }}$}

\section{Paragraph 20}

Isometric force: Despite the age difference, the young and old animals generated very similar magnitudes of isometric force on the first day of exposure $(p=0.314)$, regardless of treatment. Even though the pre-test isometric force response at the conclusion of the exposure period was not different between the young and old age groups there was a trend towards significance $(\mathrm{p}=$ 0.08), with young rodents producing $24.8 \%$ greater isometric force than their older counterparts at the conclusion of the exposure period, regardless of treatment.

\section{Paragraph 21}

Peak force $\left(\mathbf{F}_{\text {peak }}\right)$ : The peak eccentric force response was not different at the beginning of the exposure period, but differed with age following the chronic exposure $(\mathrm{p}<0.05$, Figure $1 \mathrm{a})$, with young rodents producing significantly greater peak eccentric force than their older counterparts. 
The young rodent's peak eccentric force output was $17 \%$ greater than the old rodents at the conclusion of the exposure period. In addition, treatment resulted in an increase in the young BSO- and VEH-treated rodent's peak eccentric forces following exposure $(p<0.05$, Figure $1 b)$. All other comparisons were not significant.

\section{Paragraph 22}

Minimum force $\left(\mathbf{F}_{\mathbf{m i n}}\right)$ : The minimum force response at the onset of the exposure period differed with age, with young rodents producing greater minimum force than old rodents $(\mathrm{p}<$ 0.05 , Figure 1c), regardless of treatment. The minimum force also differed with respect to age at the conclusion of the exposure period between the young and old age groups $(\mathrm{p}<0.05$, Figure 1c), with young rodents producing significantly greater minimum force than their older counterparts irrespective of treatment. The young rodent's minimum force output was $37.5 \%$ greater than the old rodents at the conclusion of the exposure period. All other comparisons were not significant.

\section{Paragraph 23}

Work: Age also influenced the ability of the dorsiflexor muscles to both absorb work (negative work) and produce work (positive work). Treatment and age had no effect on the rodent's ability to absorb work at the beginning of the exposure period. While treatment had no effect following exposure, the ability to absorb work differed with respect to age at the conclusion of the exposure period $(\mathrm{p}<0.05$, Figure $2 \mathrm{a})$, with young rodents showing a significantly greater ability to absorb work than their older counterparts. The young rodent's ability to absorb work was $21.6 \%$ greater than the old rodents at the conclusion of the exposure period. The young rodent's 
ability to produce work was $34.7 \%$ greater than the old rodents at the conclusion of the exposure period. Specifically, negative work was greater prior to exposure in the young BSO- and VEHtreated rodents compared with old BSO- and VEH-treated rodents, and negative work was also greater at the conclusion of the exposure period in the young BSO- and VEH-treated rodents compared with old BSO- and VEH-treated rodents, respectively ( $p<0.05$, Figure $2 b)$. Unlike the observation for negative work, the ability to produce work differed with age at the onset of the exposure period irrespective of treatment, with young rodents producing significantly greater work than old rodents ( $p<0.05$, Figure $2 \mathrm{c}$ ). As was the case for negative work, similar changes were observed for the ability to produce work at the conclusion of the exposure period between young and old rodents, regardless of treatment. The young rodents produced significantly greater work than their older counterparts $(\mathrm{p}<0.05$, Figure $2 \mathrm{c})$. All other comparisons were not significant.

\section{Paragraph 24}

Body weights: The young rodents significantly increased average body weight over the 4.5 week exposure period, regardless of treatment group, while the old rodents significantly decreased body weight over the same 4.5 week period, regardless of treatment.

\section{Paragraph 25}

Muscle wet-weights: Overall, young rodents had an increased muscle wet-weight when compared to old rodents, regardless of treatment ( $p<0.05$, Figure 3$)$. Following 4.5 weeks of exposure, muscle wet-weights from the exposed LTA was significantly increased compared the 
contra-lateral control RTA, irrespective of age or treatment $(p<0.05$, Figure 3$)$. All other comparisons were not significant.

\section{Paragraph 26}

Muscle Quality: Regardless of treatment, age influenced the mean value of isometric force normalized to muscle wet-weight $(0.015 \pm 0.0001 \mathrm{~N} / \mathrm{mg}$ and $0.012 \pm 0.0001 \mathrm{~N} / \mathrm{mg}$ for the young group and old group, respectively). The young group had a significantly greater isometric force normalized to muscle wet-weight in the exposed limb after the chronic SSC protocol than the old group ( $\mathrm{p}=0.05$, Figure 4$)$.

\section{Paragraph 27}

Stereological analyses of normal myofibers: Old age decreased the volume density of normal myofibers compared to younger counterparts, regardless of treatment or $\operatorname{limb}(\mathrm{p}<0.001$, Figure 5a). Additionally, despite age or limb, there was a decrease with treatment in the volume density of normal myofibers in the BSO-treated group when compared with cage-CON rodents $(\mathrm{p}<$ 0.05, Figure 5b). All other comparisons were not significant.

\section{Paragraph 28}

Stereological analyses of myofiber degeneration: Statistical analysis showed that there were no degenerative myofibers present in muscle samples analyzed from any groups.

\section{Paragraph 29}

Stereological analyses of inflammation: Aging influenced the volume density of non-cellular interstitium, indicative of edema, as old rodents showed increased NCI compared to young 
counterparts, regardless of treatment or limb $(\mathrm{p}<0.001$, Figure 5c). Further, BSO-treated rodents displayed increased NCI when compared to cage-control rodents, as age and limb had no effect $(p<0.05$, Figure 5d). Aging did not influence the response of cellular interstitium, indicative of cellular infiltrates.

\section{Paragraph 30}

Fiber cross-sectional area: Muscle cross-sectional area cut-offs were established using young and old cage-CON animals. Cross-sectional area data from young rodents showed that approximately $46 \%$ of RTA and $48 \%$ of LTA muscle fibers were located at $\geq 2,000$ um $^{2}$. Crosssectional area data from the old rodent's revealed similar results, with approximately $50 \%$ of RTA and $46 \%$ of LTA muscle fibers located at $\geq 2,000 \mathrm{um}^{2}$. Thus, a cut-off of $\geq 2,000 \mathrm{um}^{2}$ was established. Review of the histological sections is supportive of the muscle wet weight data. Representative sections from the young, VEH-treated LTA (Figure 6a) depict normal morphology and larger fibers than the corresponding young contra-lateral RTA (Figure 6b), indicative of a hypertrophic response. Even though a hypertrophic-response is present, the muscle sections from the old, VEH-treated LTA (Figure 6c) depict fibers with a blunted hypertrophic response and evidence of mild cellular infiltrates that are not evident in the samples from the non-exposed limb in either the old (Figure 6d) or young, VEH-treated rodents (Figure $6 \mathrm{~b})$. In the contra-lateral control RTA, approximately $50 \%$ of the fibers from the old, VEHtreated animals and $47 \%$ of the fibers from the young, VEH-treated rodents were $\geq 2,000 \mathrm{um}^{2}$ (Figure 8a). In the exposed LTA, approximately $61 \%$ of the fibers from the old, VEH-treated animals and $65 \%$ of the fibers from the young, VEH-treated rodents were $\geq 2,000 \mathrm{um}^{2}$ (Figure 8b). This indicates that exposure to chronic SSCs resulted in an increased percentage of larger 
fibers in the young, VEH-treated rodents (from $47 \%$ to $65 \%$ ) with a diminished shift to larger fibers in the old, VEH-treated rodents (50\% to $61 \%)$. BSO treatment resulted in similar fiber cross-sectional area shifts with respect to exposure when compared with VEH-treated rodents, regardless of age. Representative sections from the young, BSO-treated LTA (Figure 7a) depict normal morphology and larger fibers than the corresponding young contra-lateral RTA (Figure 7b), indicative of a hypertrophic response. However, the muscle sections from the old, BSOtreated LTA (Figure 7c) depict fibers with a blunted hypertrophic response compared with the contra-lateral control RTA (Figure 7d), and no evidence of cellular infiltrates that are evident in the samples from the exposed LTA in the old, VEH-treated rodents (Figure 7c). In the contralateral control RTA, approximately $50 \%$ of the fibers from the old, BSO-treated animals and $46 \%$ of the fibers from the young, BSO-treated animals were $\geq 2,000 \mathrm{um}^{2}$ (Figure $8 \mathrm{c}$ ). In the exposed LTA, approximately $62 \%$ of the fibers from the old, BSO-treated animals and $68 \%$ of the fibers from the young, BSO-treated animals were $\geq 2,000 \mathrm{um}^{2}$ (Figure $8 \mathrm{~d}$ ). This indicates that exposure to chronic SSCs resulted in an increased percentage of larger fibers in the young animals (from $46 \%$ to $68 \%$ ) with a diminished shift to larger fibers in the older animals (50\% to $62 \%)$.

\section{DISCUSSION}

\section{Paragraph 31}

To our knowledge, this is the first investigation in rodents to report increased muscle hypertrophy following glutathione depletion as a means to study the adaptive response with aging following high-intensity mechanical loading via SSCs. Novel findings reported in the present study do oppose our initial hypotheses. First, glutathione depletion, via BSO treatment, 
did not result in overt mal-adaptation in young rodents. As indicated in our methods, the dose of BSO used to deplete total glutathione was $10 \mathrm{mM}$; this resulted in total glutathione levels being depleted $\sim 65 \%$ when compared to control levels (unpublished data). Using this dosing strategy in a recent study in our lab resulted in decreased glutathione content in the TA muscle, as well as increased markers of oxidative stress and cell death in aged rodents compared with young counterparts (Hollander et al., In preparation 2007). In the current study, exacerbation of the mal-adaptive state was not observed in old BSO-treated rodents, and to the contrary, all BSOtreated rodents displayed a minimal capacity to adapt with respect to various dependent measures. It was hypothesized that depleting the internal host environment of glutathione would have increased oxidative stress, and aging would have intensified this response due the damaging and cumulative effects of ROS that evolve over time (Bejma and Ji, 1999). Surprisingly an increase in cellular infiltrates was not present (this is in contrast to what we have previously reported (Cutlip et al., 2006)), although a trend did exist in the old rodents. Thus, the attenuation of the cellular component of inflammation may have facilitated the observed adaptation with the old, exposed rodents via various cell signaling pathways. Nevertheless, we have shown that young rodents generally continue to adapt functionally and morphologically to chronic SSC exposure in the presence of glutathione depletion. Given the correct stimulus, as mentioned above, it is also apparent that there may be some residual capacity for this to occur in aging skeletal muscle as well. This may alternately be achieved by specifically and selectively conserving glutathione in metabolically active tissue and allowing for maintenance of increased oxidative stress (Leeuwenburgh and Ji, 1995) or by compensatory mechanisms following glutathione depletion. Thus, it may be concluded that glutathione depletion in rodents exposed 
to high-intensity mechanical loading via SSC exposure does not directly induce a mal-adaptive state.

\section{Paragraph 32}

The differences observed with respect to initial and final isometric and dynamic performance measures in the current investigation with those published by our lab recently (Cutlip et al., 2006) may be the result of the variation we observed in the size of the TA muscle fibers.

Previously, our morphometric data showed that young and old Fischer Hybrid rodent TA muscle fibers (of the same age in the current study) were smaller in size regardless of training status than observed in this study. This may have resulted in the disparity we observed between both the initial force generating capacity of the rodents in this study, as well as the adaptive/mal-adaptive response to exposure. However, the observation that the isometric performance of both the young and old animals was similar at the start of the exposure period is critical in acknowledging, because this establishes that age did not have an effect on the ability of untrained animals to generate isometric force at the onset of the exposure protocol. Data reported for the VEH-treated rodents is consistent with results from our lab (Cutlip et al., 2006) as well as others (Brooks and Faulkner, 1994); Brooks et al., reported no difference in isometric or concentric performance in old and young muscles of mice and even increased performance during lengthening contractions in old mice. Even though old rodents in the current study did not increase functional performance, the ability to generally maintain static and dynamic performance is an important finding. Given that following this type of mechanical exposure, adaptation does occur, recapitulation of the regenerative pathway in skeletal muscle would be a plausible mechanism for inducing this adaptive response. In a very recent study in our lab, we 
have illustrated that an acute exposure of the current protocol results in minimal degenerative myofibers $(<1 \%)$ temporally, regardless of age, however immunolabeling for developmental myosin heavy chain 5 days following exposure revealed that there is a diminished response in aged rodents when compared to young counterparts (Baker et al., In review 2007). In this study aged rodents did increase expression of developmental myosin in the exposed limb compared to the control limb, suggesting that the initial signal for regeneration is retained but the capacity to respond as young rodents do to the same stimulus is reduced significantly. These findings support the view that although aging muscles have a delayed recovery following acute exposure, muscles of old rodents do retain some ability to adapt. Brooks and colleagues (Brooks et al., 2001) have reported this finding and shown that when damaging eccentric muscle actions are incorporated into a repetitive exposure model, there is evidence that age does not have a deleterious effect on adaptation when this eccentric protocol is administered once per week in vivo. The exposure paradigm used in the current study resulted in an average increase in isometric force of $4.2 \%$ above the pre-test force in young, VEH-treated rodents, while producing a $12.9 \%$ deficit in old, VEH-treated rodents. The same general response was observed in the young and old rodents receiving BSO treatment with respect to isometric performance. Even though this was not significant, the general pattern of adaptation/mal-adaptation was observed with aging, as we have previously shown (Cutlip et al., 2006). Furthermore, peak eccentric force increased in both the young, VEH-treated (12.1\%) and young, BSO-treated (17.9\%) rodents but did not increase in the old, VEH- or BSO-treated rodents. The data reported here for our VEH rodents is in general agreement with Brooks et al. (Brooks et al., 2001) who reported a significant increase in peak force by week 6 for both adult and old animals over baseline values in mice. Disparities between our current performance data and that which has been reported 
previously may be related to differences between and within species and/or strains, as well as to differences we have noted with respect to muscle fiber size heterogeneity within this strain.

\section{Paragraph 33}

Stereological analyses detected no degenerative myofibers or cellular infiltrates following the terminal session in the current study, but did show a significant increase in the volume of noncellular interstitium, indicative of edema, in the muscles of old rodents. Further, degeneration and cellular components of inflammation do not appear to be influenced by glutathione depletion; however the BSO-treated rodents did have increased measures of NCI. Collectively, the precise composition and magnitude of the general exposure response (degeneration, inflammation, and swelling) may be an important factor in signaling skeletal muscle adaptation (Tidball, 2005). Thus, an important factor involving the remodeling of skeletal muscle during adaptation continues to indicate that modifications made to the interstitial space are critical, and the inability of old rodents to fully adapt may be significantly influenced by the internal host environment as hypothesized by others (Conboy et al., 2005). Furthermore, the adaptation observed in this study involving the old rodents may have resulted from attenuation of the cellular interstitial response that we have recently reported (Cutlip et al., 2006). By decreasing the cellular component of inflammation, a more favorable host environment may have resulted in terms of skeletal muscle adaptation. In addition we are again reporting the absence of degenerative myofibers following high-intensity mechanical loading, which supports a recent investigation from our lab (Cutlip et al., 2006), where the exposed muscles of rodents did not exhibit any degenerative myofiber response. This may validate the assumption that not all highintensity mechanical loading leads to overt skeletal muscle injury. This is especially important 
in light of our model, which uses supra-maximal electrical stimulation to elicit muscle contractions. Even though this type of muscle activation rarely is encountered in human populations, we are continuing to find that not all muscle contractions result in muscle degeneration (even those comprised of "damaging eccentric contractions"). We postulate that human muscular contractions undergoing chronic adaptation, even those individuals encountering maximal exposure intensity, rarely undergo overt muscle degeneration.

\section{Paragraph 34}

The data in the present study clearly show that exposed muscles in young rodents adapt to chronic SSC exposure by increasing muscle mass $(20.8 \%$ increase over the contra-lateral control muscle for young, VEH-treated and $21.9 \%$ increase over the contra-lateral control muscle for young, BSO-treated), while this response was diminished with the old rodents $(10.3 \%$ increase over the contra-lateral control muscle for old, VEH-treated and 9.4\% increase over the contralateral control muscle for old, BSO-treated). The increase in muscle wet-weight could have resulted from and be attributed to chronic edema, but based on our findings, we do not believe this is the case. Results from our muscle cross-sectional area data support the observation that there was a substantial degree of myofiber hypertrophy in the young, exposed animals as evidenced by a shift to larger fibers (a shift from $46 \%$ to $68 \%$ of fibers $\geq 2,000 \mathrm{um}^{2}$ ). In addition, stereological analysis of exposed muscle from young rats showed no increases in NCI response, (i.e., no edema). On the contrary, the observed increase in the NCI response of old rodents, indicative of edema, may have prevented the decline in muscle-wet-weight, however morphological data support that myofibers in old rodents were increasing fiber area (a shift from $50 \%$ to $62 \%$ of fibers $\geq 2,000 \mathrm{um}^{2}$ ). Despite previous reports that indicate skeletal muscles in 
old animals are more susceptible to injury (Brooks and Faulkner, 1996, Zerba et al., 1990), and recover more slowly (McBride et al., 1995, Brooks and Faulkner, 1990) from a single exposure to injurious contractions, in support of our findings is evidence that old animals can be conditioned for protection from contraction-induced myofiber injury (Brooks et al., 2001). Muscle hypertrophy and improvements in force production occurs in response to constant or chronic loading in aged animals, although the extent of muscle enlargement is attenuated relative to young animals (Alway, 1995, Alway et al., 2002, Carson et al., 1995, Klitgaard et al., 1989a, Klitgaard et al., 1989b, Lowe et al., 1998). Since myofiber cross sectional area and stereology data from the contra-lateral limbs of both groups were not different, this suggests that there was not a different systemic response. The lack of myofiber degeneration in the exposed limbs of the old groups suggests that the diminished capacity to adapt is not due to fiber degeneration, but necessitates the need for future research into the causal factors influencing the adaptive response with aging.

\section{Paragraph 35}

As well as age, several factors may explain the difference in performance, physiological, and morphological measures between the two groups during the current chronic exposure and differences observed with a recent investigation from our lab (Cutlip et al., 2006). 1) The mode of exposure may significantly contribute to changes observed following repetitive exposure (anaerobic versus aerobic exposures). 2) The older animals may not have tolerated the repeated exposure to isoflurane as well as their younger counterparts, which could have affected contractile performance. 3) However, performance data clearly shows that old rodents have some capacity to adapt and that an alternate explanation to the differences observed may be influenced 
by inherent variability within or between cohorts of an animal strain or between species examined. Recently, we have demonstrated that old animal's mal-adapt to the identical exposure paradigm both functionally, physiologically, and morphologically. In the current study, overt mal-adaptation that we observed previously was not as striking.

\section{Paragraph 36}

Our findings suggest that glutathione depletion in rodents exposed to repetitive SSCs does not induce a mal-adaptive state. Yet, we are unable to rule out completely the involvement of other oxidative pathways that may be influential in the producing adaptation/mal-adaptation to chronic SSC exposures. Isolating elements such as increased oxidative stress and/or increased inflammation in skeletal muscle under repetitive high-intensity mechanical loading that may influence the response of the oxidant/antioxidant profile continues to be of major importance when designing preventative strategies that will need to manage skeletal muscle mal-adaptation in workplace and recreation settings. Difficulties exist in drawing definitive conclusions as to the influence glutathione has on adaptation with aging due to disparities that exist between modes of exposure. Also, there is a paucity of studies examining the role glutathione and other antioxidants have on skeletal muscle adaptation with mechanical loading and aging. In the present study, we show that adaptive changes are diminished in old rodents, and aging negatively influences this adaptive response by limiting performance as well as physiological adaptation; however glutathione depletion does not adversely effect nor exacerbate this adaptive profile. 


\section{REFERENCES}

ALWAY, S. E. (1995) Slowing of contractile properties in quail skeletal muscle with aging. $J$ Gerontol A Biol Sci Med Sci, 50A, B26-33.

ALWAY, S. E., DEGENS, H., KRISHNAMURTHY, G. \& SMITH, C. A. (2002) Potential role for Id myogenic repressors in apoptosis and attenuation of hypertrophy in muscles of aged rats. Am J Physiol Cell Physiol, 283, C66-76.

BAKER, B. A., MERCER, R. R., GERONILLA, K. B., KASHON, M. L., MILLER, G. R. \& CUTLIP, R. G. (2006a) Stereological analysis of muscle morphology following exposure to repetitive stretch-shortening cycles in a rat model. Appl Physiol Nutr Metab, 31, $167-$ 79.

BAKER, B. A., RAO, K. M., MERCER, R. R., GERONILLA, K. B., KASHON, M. L., MILLER, G. R. \& CUTLIP, R. G. (2006b) Quantitative histology and MGF gene expression in rats following SSC exercise in vivo. Med Sci Sports Exerc, 38, 463-71.

BAKER, B. A., HOLLANDER, M. S., MERCER, R. R., KASHON, M. L., \& CUTLIP, R. G. (In review 2007) Adaptive stretch-shortening contractions: diminished regenerative capacity with aging.

BEJMA, J. \& JI, L. L. (1999) Aging and acute exercise enhance free radical generation in rat skeletal muscle. J Appl Physiol, 87, 465-70.

BROOKS, S. V. \& FAULKNER, J. A. (1990) Contraction-induced injury: recovery of skeletal muscles in young and old mice. Am J Physiol, 258, C436-42.

BROOKS, S. V. \& FAULKNER, J. A. (1994) Isometric, shortening, and lengthening contractions of muscle fiber segments from adult and old mice. Am J Physiol, 267, C50713.

BROOKS, S. V. \& FAULKNER, J. A. (1996) The magnitude of the initial injury induced by stretches of maximally activated muscle fibres of mice and rats increases in old age. $J$ Physiol, 497 ( Pt 2), 573-80.

BROOKS, S. V., OPITECK, J. A. \& FAULKNER, J. A. (2001) Conditioning of skeletal muscles in adult and old mice for protection from contraction-induced injury. J Gerontol A Biol Sci Med Sci, 56, B163-71. 
CARSON, J. A., ALWAY, S. E. \& YAMAGUCHI, M. (1995) Time course of hypertrophic adaptations of the anterior latissimus dorsi muscle to stretch overload in aged Japanese quail. J Gerontol A Biol Sci Med Sci, 50, B391-8.

CLARKSON, P. M. \& HUBAL, M. J. (2002) Exercise-induced muscle damage in humans. Am J Phys Med Rehabil, 81, S52-69.

CONBOY, I. M., CONBOY, M. J., WAGERS, A. J., GIRMA, E. R., WEISSMAN, I. L. \& RANDO, T. A. (2005) Rejuvenation of aged progenitor cells by exposure to a young systemic environment. Nature, 433, 760-4.

CUTLIP, R. G., BAKER, B. A., GERONILLA, K. B., MERCER, R. R., KASHON, M. L., MILLER, G. R., MURLASITS, Z. S. \& ALWAY, S. E. (2006) Chronic exposure of stretch-shortening contractions results in skeletal muscle adaptation in young rats and maladaptation in old rats. Applied Physiology, Nutrition and Metabolism, 31, 573-587.

CUTLIP, R. G., GERONILlA, K. B., BAKER, B. A., CHETLIN, R. D., HOVER, I., KASHON, M. L. \& WU, J. Z. (2005) Impact of stretch-shortening cycle rest interval on in vivo muscle performance. Med Sci Sports Exerc, 37, 1345-55.

CUTLIP, R. G., GERONILLA, K. B., BAKER, B. A., KASHON, M. L., MILLER, G. R. \& SCHOPPER, A. W. (2004) Impact of muscle length during stretch-shortening contractions on real-time and temporal muscle performance measures in rats in vivo. $J$ Appl Physiol, 96, 507-16.

CUTLIP, R. G., STAUBER, W. T., WILLISON, R. H., MCINTOSH, T. A. \& MEANS, K. H. (1997) Dynamometer for rat plantar flexor muscles in vivo. Medical \& Biological Engineering \& Computing, 35, 540-3.

DAVIS, J., KAUFMAN, K. R. \& LIEBER, R. L. (2003) Correlation between active and passive isometric force and intramuscular pressure in the isolated rabbit tibialis anterior muscle. $J$ Biomech, 36, 505-12.

GERONILLA, K. B., MILLER, G. R., MOWREY, K. F., WU, J. Z., KASHON, M. L., BRUMBAUGH, K., REYNOLDS, J., HUBBS, A. \& CUTLIP, R. G. (2003) Dynamic force responses of skeletal muscle during stretch-shortening cycles. Eur J Appl Physiol, 90, 144-53.

HOLLANDER, M.S. (In preparation 2007)

INGALLS, C. P., WARREN, G. L., LOWE, D. A., BOORSTEIN, D. B. \& ARMSTRONG, R. B. (1996) Differential effects of anesthetics on in vivo skeletal muscle contractile function in the mouse. Journal of Applied Physiology, 80, 332-40. 
INGALLS, C. P., WARREN, G. L., WILliAMS, J. H., WARD, C. W. \& ARMSTRONG, R. B. (1998) E-C coupling failure in mouse EDL muscle after in vivo eccentric contractions. $J$ Appl Physiol, 85, 58-67.

KLITGAARD, H., BRUNET, A., MATON, B., LAMAZIERE, C., LESTY, C. \& MONOD, H. (1989a) Morphological and biochemical changes in old rat muscles: effect of increased use. J Appl Physiol, 67, 1409-17.

KLITGAARD, H., MARC, R., BRUNET, A., VANDEWALLE, H. \& MONOD, H. (1989b) Contractile properties of old rat muscles: effect of increased use. J Appl Physiol, 67, 1401-8.

LEEUWENBURGH, C. \& JI, L. L. (1995) Glutathione Depletion in Rested and Exercised Mice: Biochemical Consequence and Adaptation. Arch Biochem Biophys, 316, 941-949.

LOWE, D. A., LUND, T. \& ALWAY, S. E. (1998) Hypertrophy-stimulated myogenic regulatory factor mRNA increases are attenuated in fast muscle of aged quails. Am J Physiol, 275, C155-62.

MARTENSSON, J., MEISTER, A. (1989) Mitochondrial damage in muscle occurs after marked depletion of glutathione and is prevented by giving glutathione monoester. Proc Natl Acad Sci U S A, 86, 471-475.

MCARDLE, A., PATTWELL, D., VASILAKI, A., GRIFFITHS, R. D. \& JACKSON, M. J. (2001) Contractile activity-induced oxidative stress: cellular origin and adaptive responses. Am J Physiol Cell Physiol, 280, 621-627.

MCBRIDE, J. M., KRAEMER, W.J., TRIPLETT-MCBRIDE, T., SEBASTIANELLI, W. (1998) Effect of resistance exercise on free radical production. Med Sci Sports Exerc, 30, 67-72.

MCBRIDE, T. A., GORIN, F. A. \& CARLSEN, R. C. (1995) Prolonged recovery and reduced adaptation in aged rat muscle following eccentric exercise. Mech Ageing Dev, 83, 185200.

MEISTER, A. (1991) Glutathione deficiency produced by inhibition of its synthesis, and its reversal; applications in research and therapy. Pharmac. Ther., 51.

STAUBER, W. T. (2004) Factors involved in strain-induced injury in skeletal muscles and outcomes of prolonged exposures. J Electromyogr Kinesiol, 14, 61-70.

TIDBALL, J. G. (2005) Inflammatory processes in muscle injury and repair. Am J Physiol Regul Integr Comp Physiol, 288, 345-353. 
UCHIYAMA, S., TSUKAMOTO, H., YOSHIMURA, S. \& TAMAKI, T. (2006) Relationship between oxidative stress in muscle tissue and weight-lifting-induced muscle damage. Eur J Physiol, 452, 109-116.

UNDERWOOD, E. E. (1970) Quantatative Stereology, Reading, MA, Addison-Wesley Publishing Co.

URSO, M. L. \& CLARKSON, P. M. (2003) Oxidative stress, exercise, and antioxidant supplementation. Toxicology, 189, 41-54.

WARREN, G. L., HAYES, D. A., LOWE, D. A. \& ARMSTRONG, R. B. (1993) Mechanical factors in the initiation of eccentric contraction-induced injury in rat soleus muscle. $J$ Physiol, 464, 457-75.

WARREN, G. L., HAYES, D. A., LOWE, D. A., GUO, W. \& ARMSTRONG, R. B. (1991) Mechanical Factors in Exercise-Induced Muscle Injury. FASEB Journal, 5.

WEIBEL, E. R. (1972) The value of stereology in analysing structure and function of cells and organs. J Microsc, 95, 3-13.

WEIBEL, E. R. (1974) Selection of the best method in stereology. J Microsc, 100, 261-9.

WEIBEL, E. R. (1975) Quantitation in morphology: possibilities and limits. Beitr Pathol, 155, 117.

WILLEMS, M. E. \& STAUBER, W. T. (2001) Force deficits after repeated stretches of activated skeletal muscles in female and male rats. Acta Physiol Scand, 172, 63-7.

ZERBA, E., KOMOROWSKI, T. E. \& FAULKNER, J. A. (1990) Free radical injury to skeletal muscles of young, adult, and old mice. Am J Physiol, 258, C429-35. 


\section{FIGURE LEGENDS}

Figure 1: Single SSC force parameters from the single SSCs performed prior to each SSC protocol. A) Peak force $\left(\mathrm{F}_{\text {peak }}\right)$ of the young and old groups at the beginning of the exposure period and the conclusion of the chronic exposure period. ** Significant effect of aging ( $\mathrm{p}$ $<0.05)$. Data are reported as mean values $+/$ - standard error. B) Peak force $\left(\mathrm{F}_{\text {peak }}\right)$ of the young $\mathrm{BSO}$ and $\mathrm{VEH}$ groups at the beginning of the exposure period and the conclusion of the chronic exposure period. * Significant effect of exposure $(p<0.05)$. Data are reported as mean values $+/-$ standard error. C) Minimum force $\left(\mathrm{F}_{\min }\right)$ of the young and old groups at the beginning of the exposure period and the conclusion of the chronic exposure period. ** Significant effect of aging $(\mathrm{p}<0.05)$. Data are reported as mean values $+/$ - standard error.

Figure 2: Single SSC work parameters from the single SSCs performed prior to each SSC protocol. A) The ability to absorb work (negative work) exhibited by the young and old age groups at the beginning of the exposure period and the conclusion of the chronic exposure period. ** Significant effect of aging $(\mathrm{p}<0.05)$. Data are reported as mean values $+/$ - standard error. B) Ability to absorb negative work at the beginning and following the chronic exposure period in the young BSO and VEH groups * Significant effect of exposure $(p<0.05)$. Data are reported as mean values +/- standard error. C) The ability to produce work (positive work) exhibited by the young and old age groups at the beginning of the exposure period and the conclusion of the chronic exposure period. ** Significant effect of aging $(p<0.05)$. Data are reported as mean values $+/$ - standard error. 
Figure 3: Muscle wet-weights of the exposed LTA and contra-lateral control RTA muscle were standardized to tibia length (TL) for young and old rodents following the chronic exposure period. * Significant effect of limb $(\mathrm{p}<0.05)$. ** Significant effect of aging $(\mathrm{p}<0.05)$. Data are reported as mean values $+/$ - standard error.

Figure 4: Isometric force normalized to muscle wet-weight of the tibialis anterior for young and old rodents following the chronic exposure period $(\mathrm{N} / \mathrm{mg})$. ** Significant effect of aging $(\mathrm{p}=$ 0.05). Data are reported as mean values $+/$ - standard error.

Figure 5: A \& B) The volume density (\% tissue fraction) of normal myofibers in the young and old rodents' exposed LTA and contra-lateral control RTA muscle was determined using standard stereological analyses. * Significant effect of treatment $(\mathrm{p}<0.05) . * *$ Significant effect of aging $(\mathrm{p}<0.001)$. Data are reported as mean values $+/-$ standard error. $\mathbf{C} \& \mathbf{D})$ The volume density $(\%$ tissue fraction) of non-cellular interstitium (NCI) in the young and old rodents' exposed LTA and contra-lateral control RTA muscle was determined using standard stereological analyses. * Significant effect of treatment $(\mathrm{p}<0.05)$. ** Significant effect of aging $(\mathrm{p}<0.001)$. Data are reported as mean values $+/$ - standard error.

Figure 6: Hematoxylin and Eosin stained sections of the exposed LTA and contra-lateral control RTA muscle from young, vehicle-treated (Figures $6 \mathrm{a}$ and $6 \mathrm{~b}$ ) and old, vehicle-treated rodents (Figures 6c and 6d), respectively. All micrographs shown are representative of the overall groups and are captured at $40 \mathrm{X}$ magnification. 
Figure 7: Hematoxylin and Eosin stained sections of the exposed LTA and contra-lateral control RTA muscle from young, BSO-treated (Figures 7a and 7b) and old, BSO-treated rodents (Figures 7c and 7d), respectively. All micrographs shown are representative of the overall groups and are captured at $40 \mathrm{X}$ magnification.

Figure 8: A) Mean cross-sectional area $\left(\mathrm{CSA}, \mu \mathrm{m}^{2}\right)$ distribution in young and old VEH-treated contra-lateral control RTA muscle. The frequency histogram depicts the frequency of fibers, which lie above/below our cut-off of 2,000 $\mathrm{um}^{2}$. Approximately $50 \%$ of the fibers from the older rodents and $47 \%$ of the fibers from the younger rodents were $\geq 2,000 \mathrm{um}^{2}$. B) Mean crosssectional area $\left(\mathrm{CSA}, \mu \mathrm{m}^{2}\right)$ distribution in young and old VEH-treated exposed LTA muscle. Approximately $61 \%$ of the fibers from the older rodents and $65 \%$ of the fibers from the younger rodents were $\geq 2,000 \mathrm{um}^{2}$. C) Mean cross-sectional area $\left(\mathrm{CSA}, \mu \mathrm{m}^{2}\right)$ distribution in young and old BSO-treated contra-lateral control RTA muscle. Approximately 50\% of the fibers from the older rodents and $47 \%$ of the fibers from the younger rodents were $\geq 2,000 \mathrm{um}^{2}$. D) Mean crosssectional area $\left(\mathrm{CSA}, \mu^{2}\right)$ distribution in young and old BSO-treated exposed LTA muscle. Approximately $61 \%$ of the fibers from the older rodents and $65 \%$ of the fibers from the younger rodents were $\geq 2,000 \mathrm{um}^{2}$. Regardless of treatment, note the existing population of small fibers $\left(\leq 1,000 \mathrm{um}^{2}\right)$ in the old rodent's muscle. 
Figure 1a

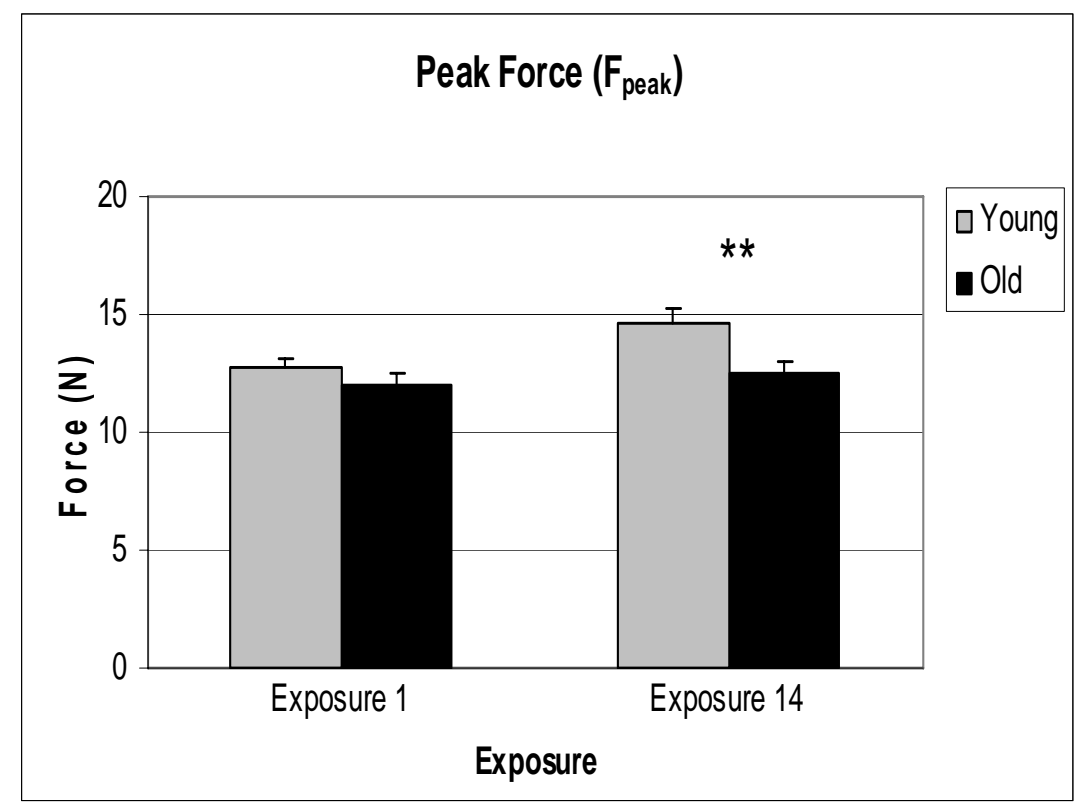

Figure 1b

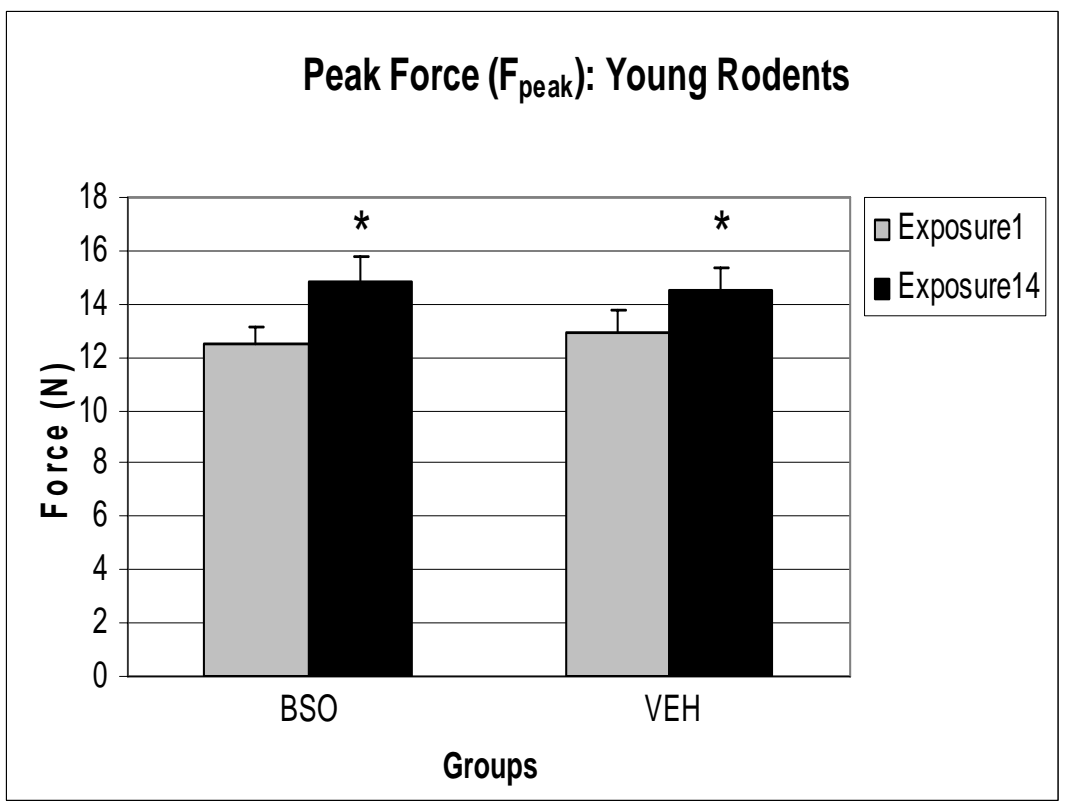


Figure 1c

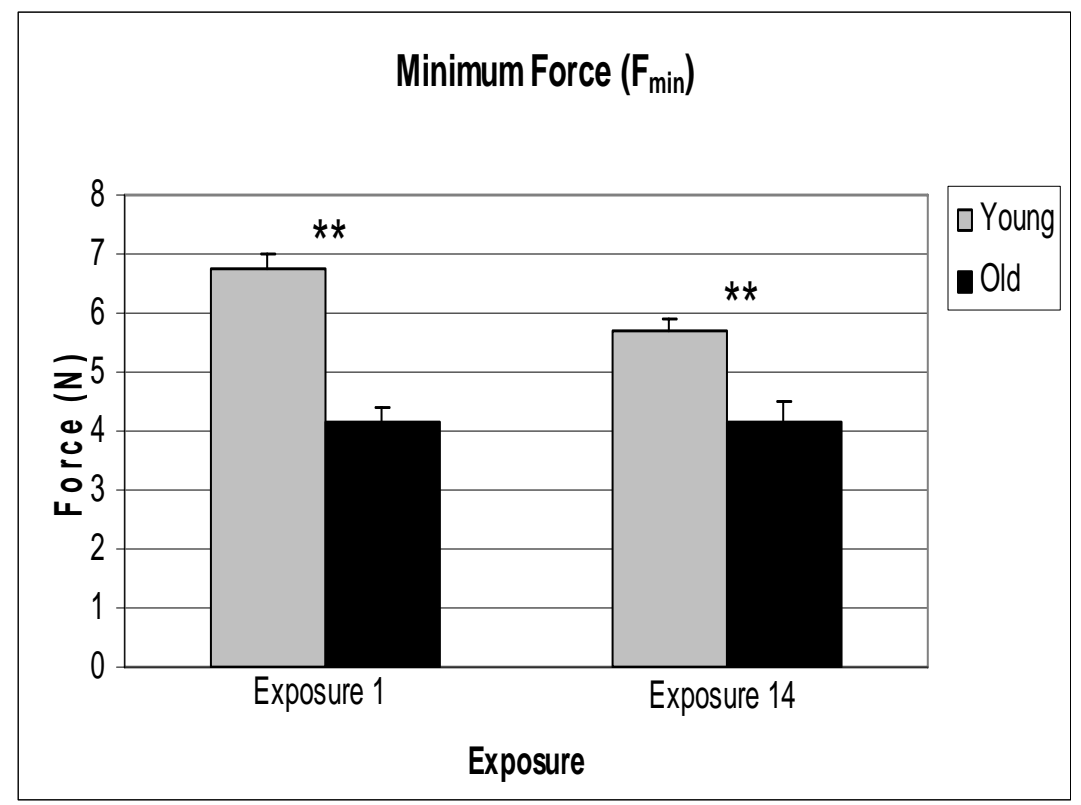


Figure 2a

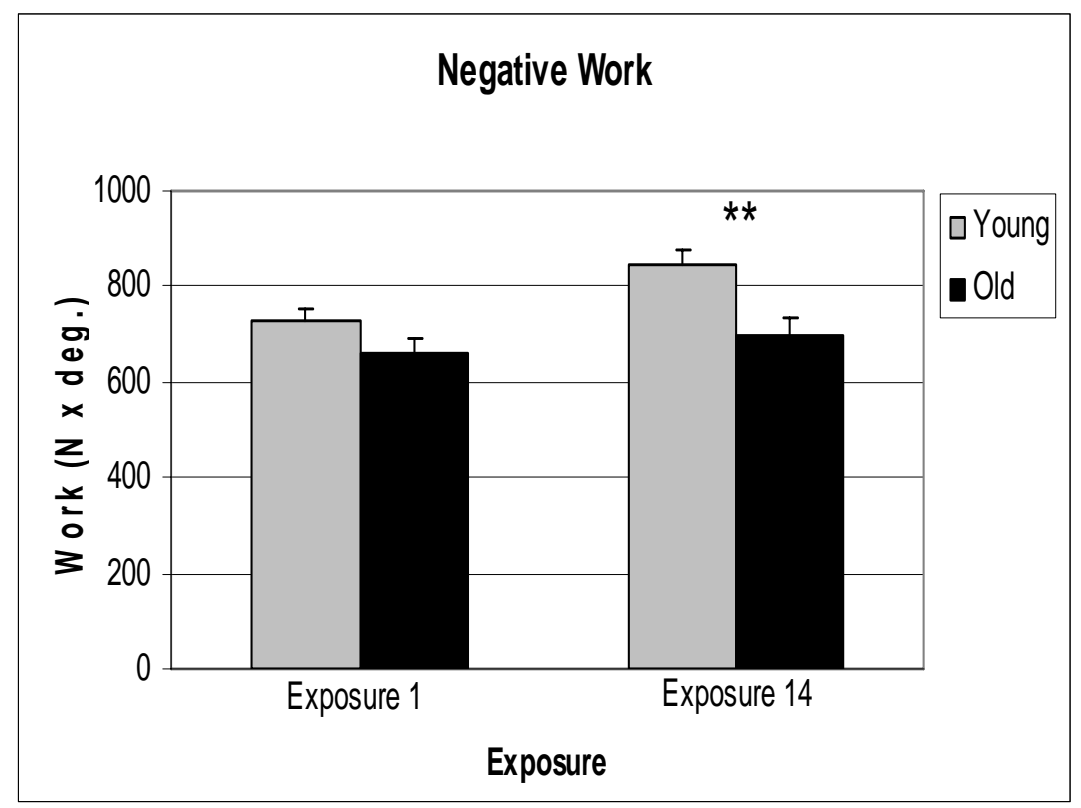

Figure 2b

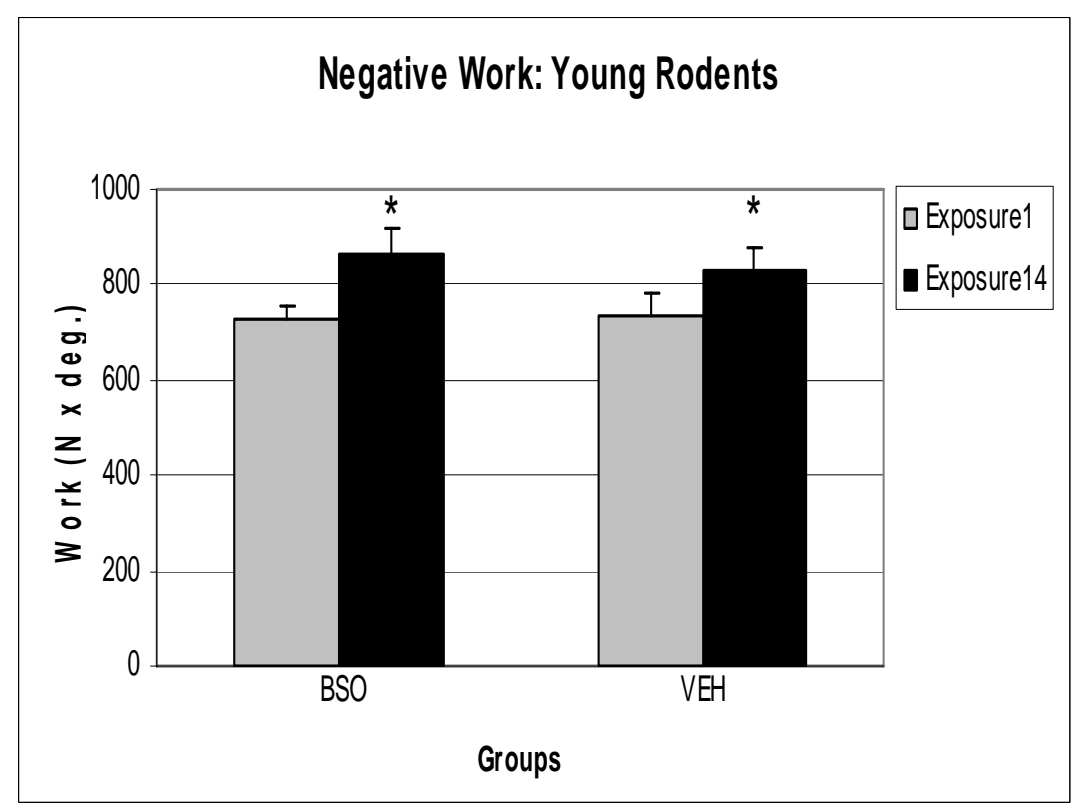


Figure 2c

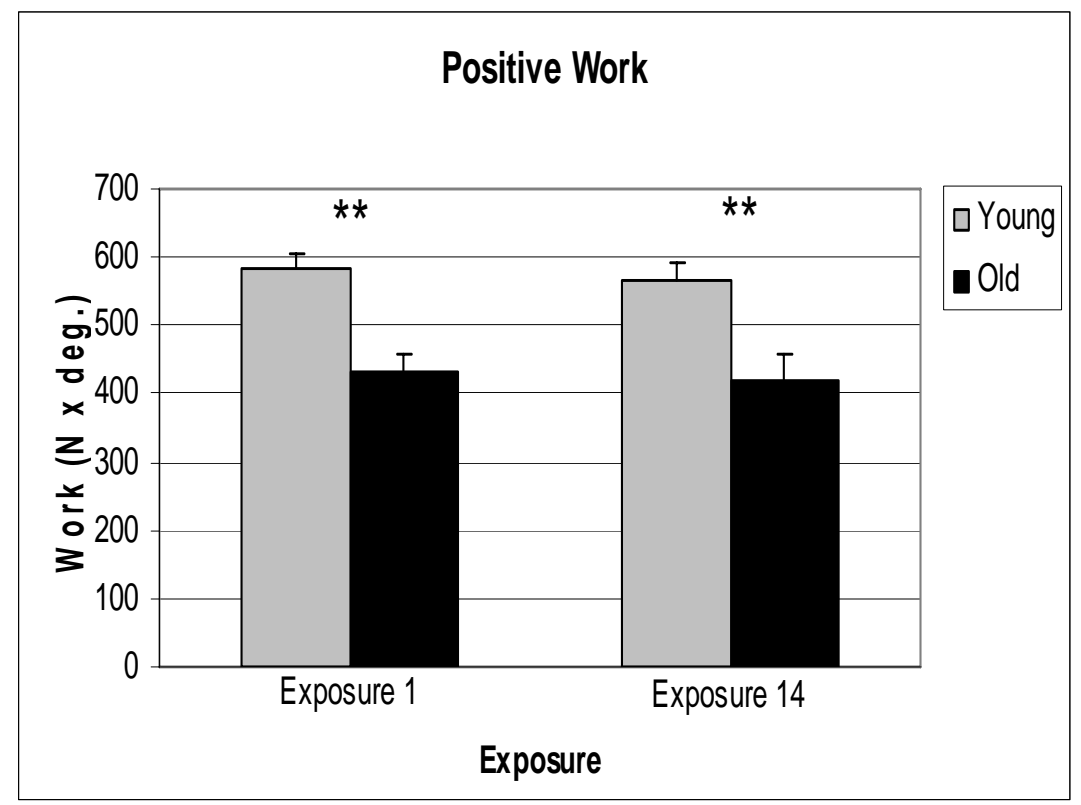


Figure 3

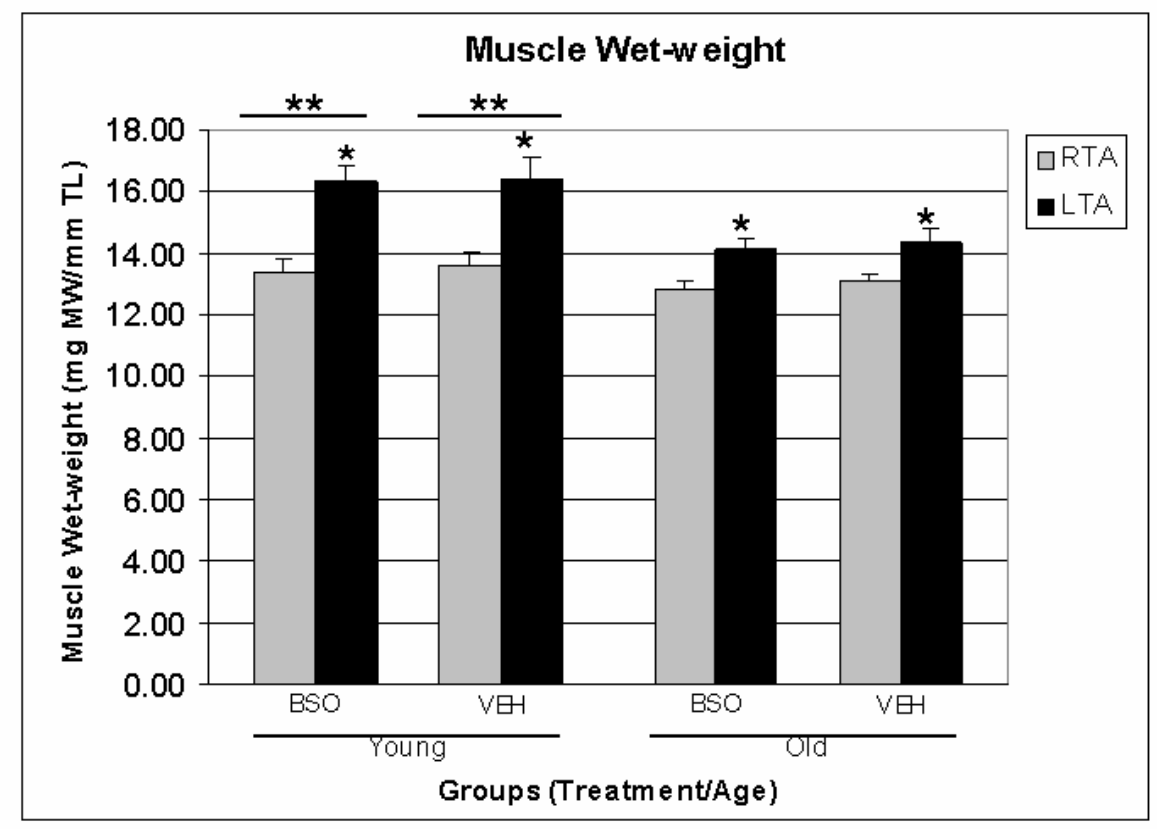


Figure 4

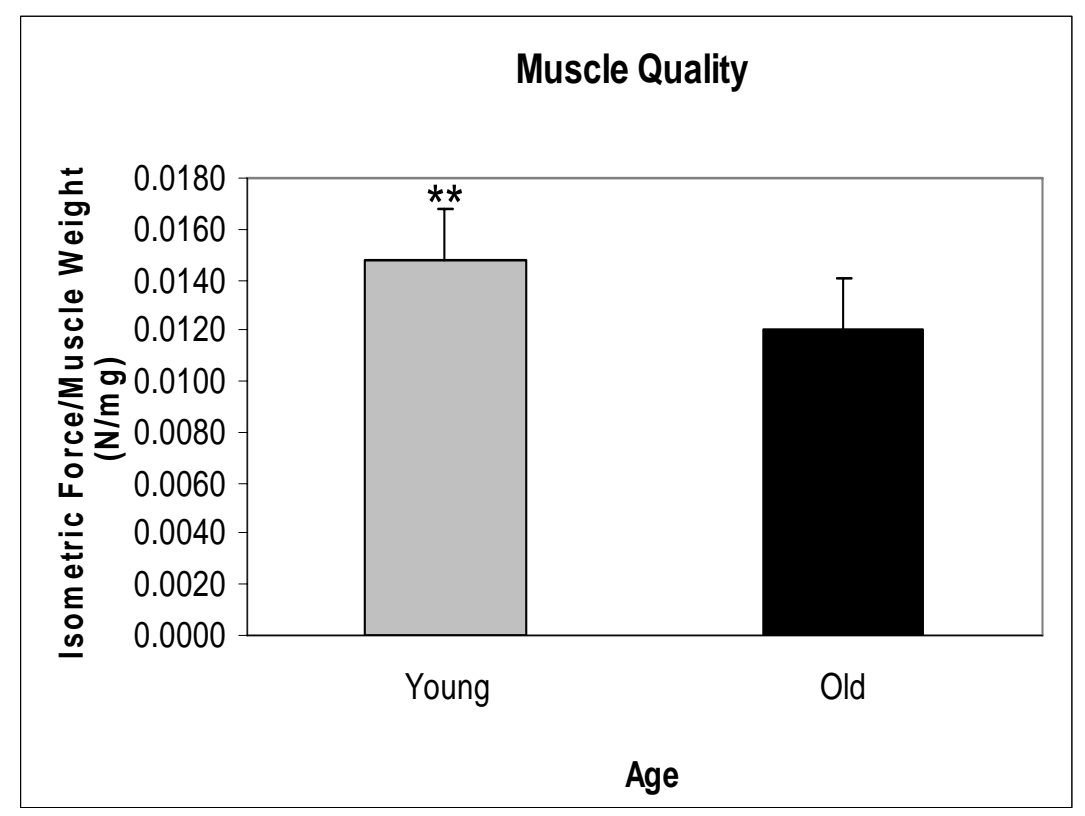




\section{Figure 5a}

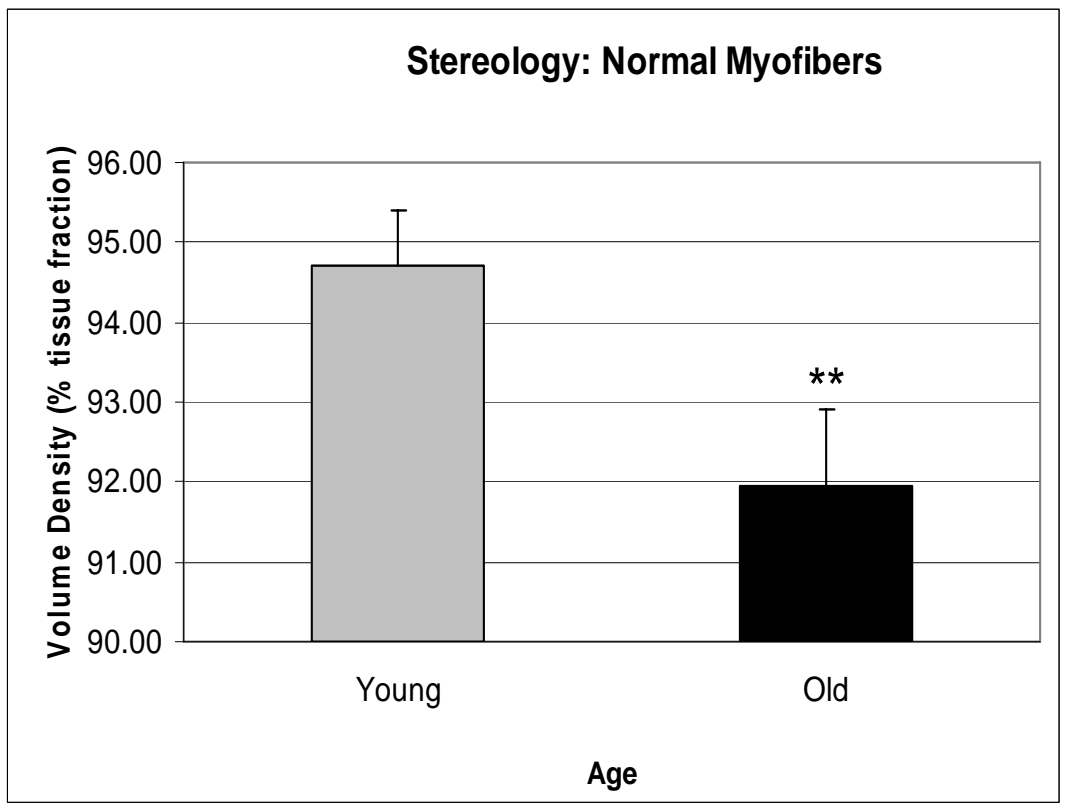

Figure 5b

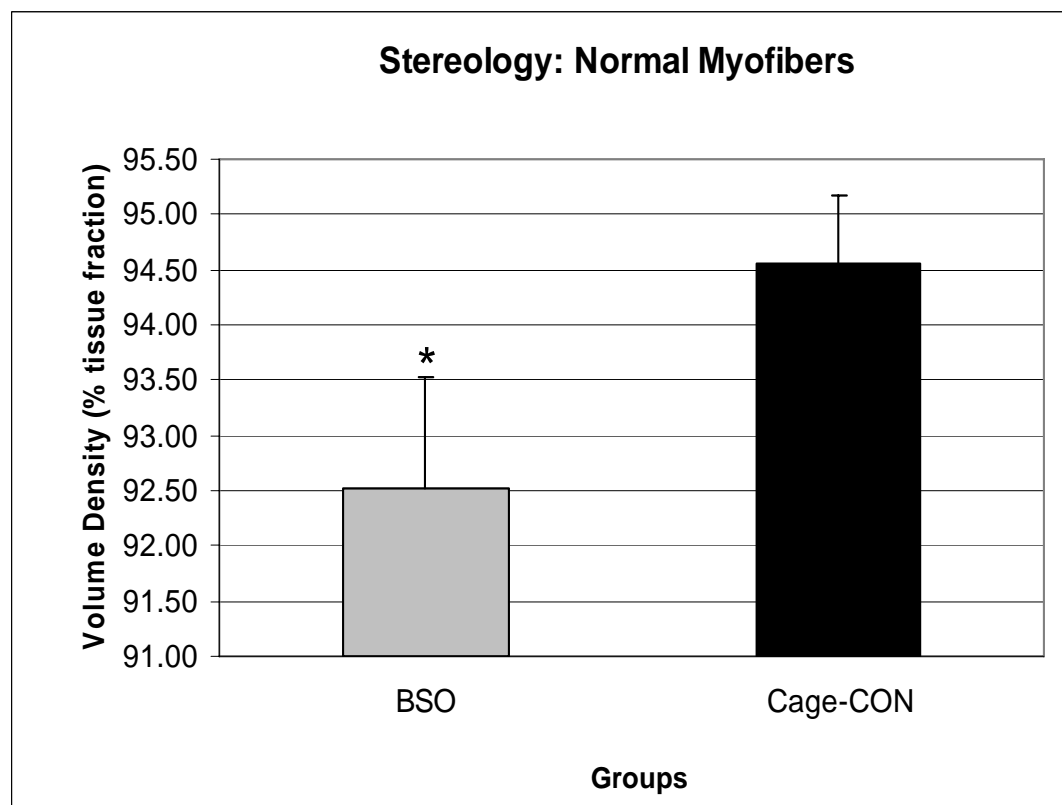




\section{Figure 5c}

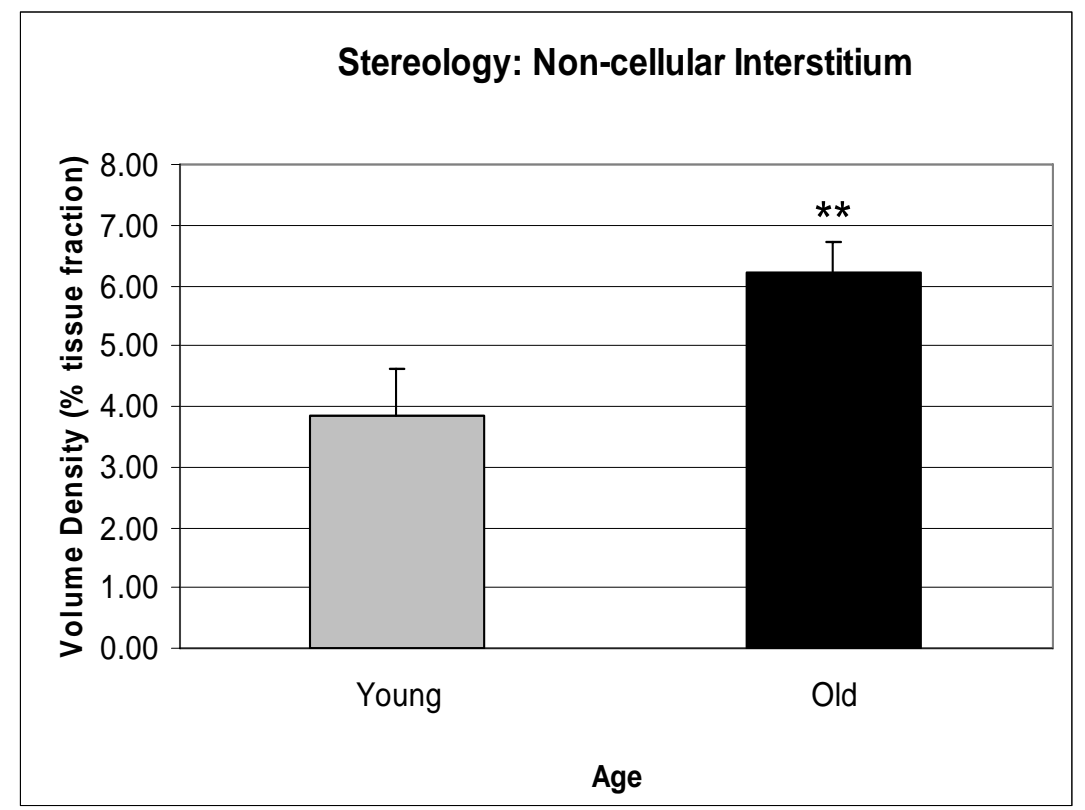

Figure 5d

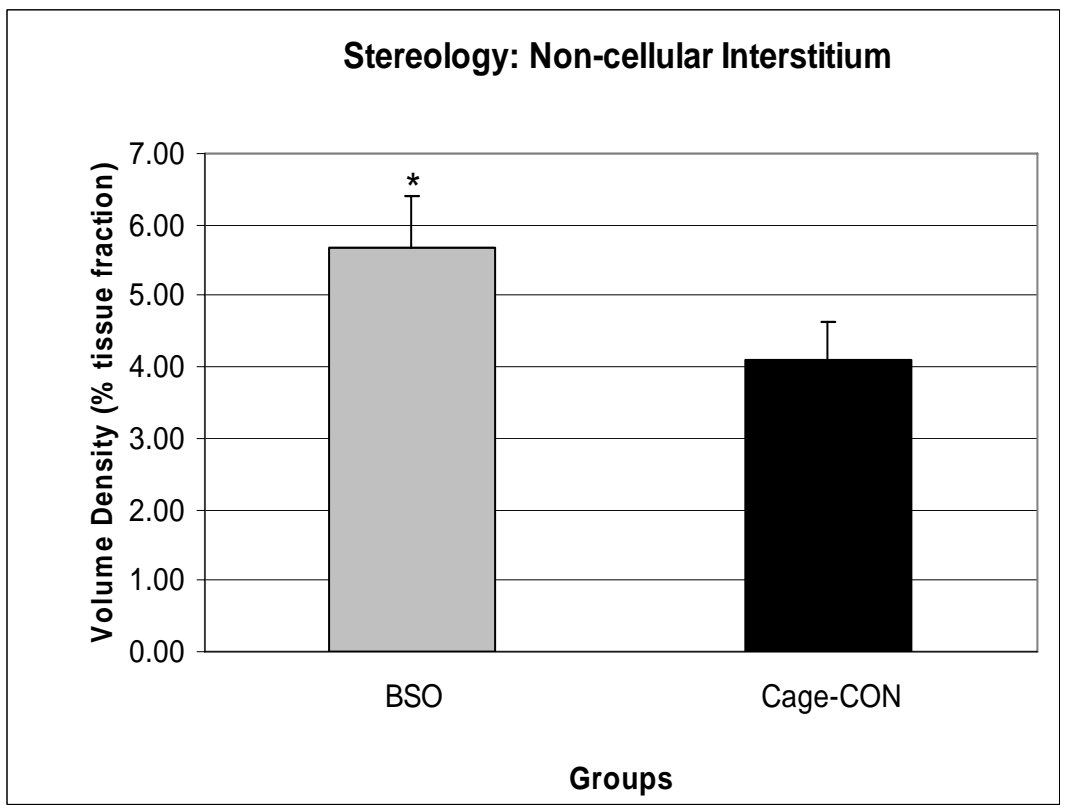


Figure 6

A) Young LTA

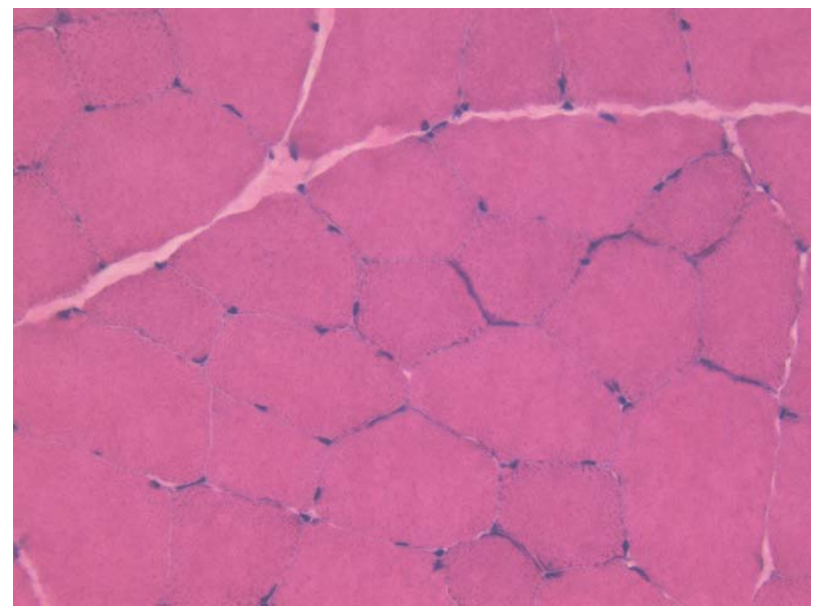

C) Old LTA

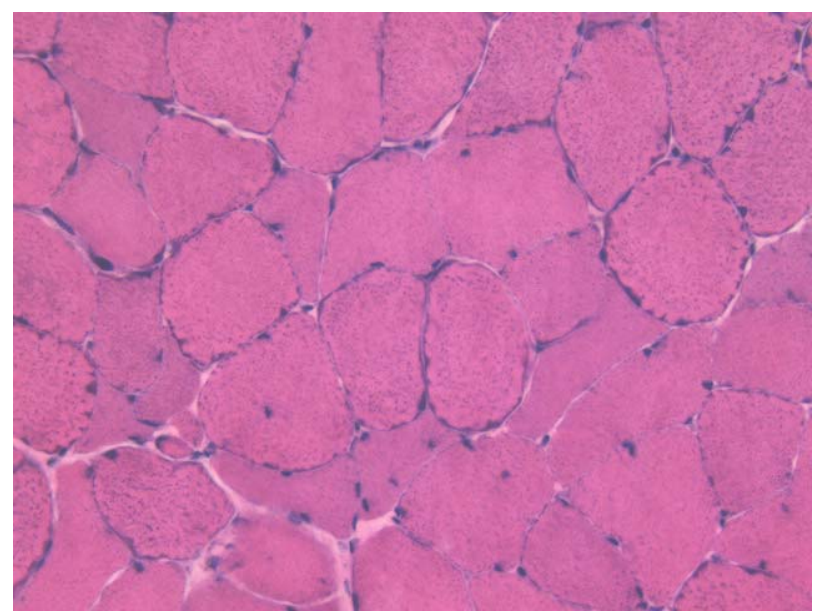

B) Young RTA

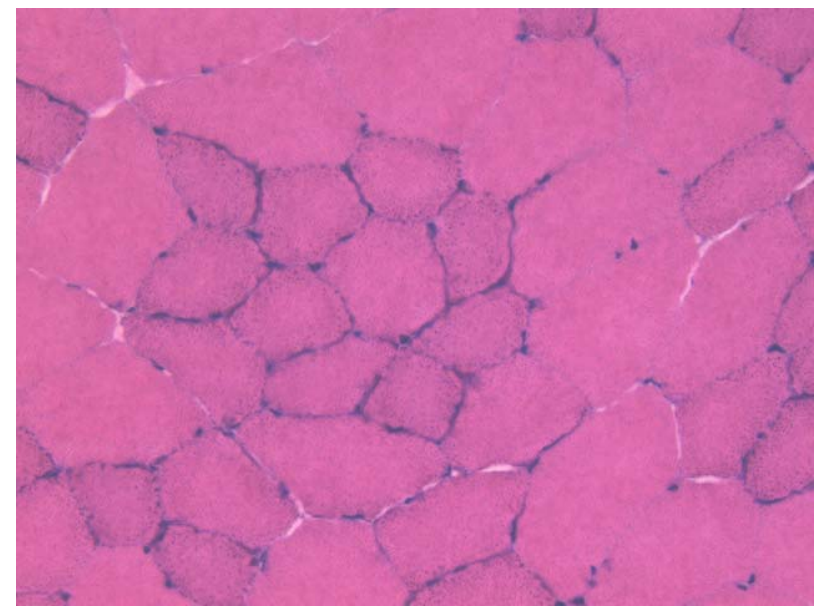

D) Old RTA

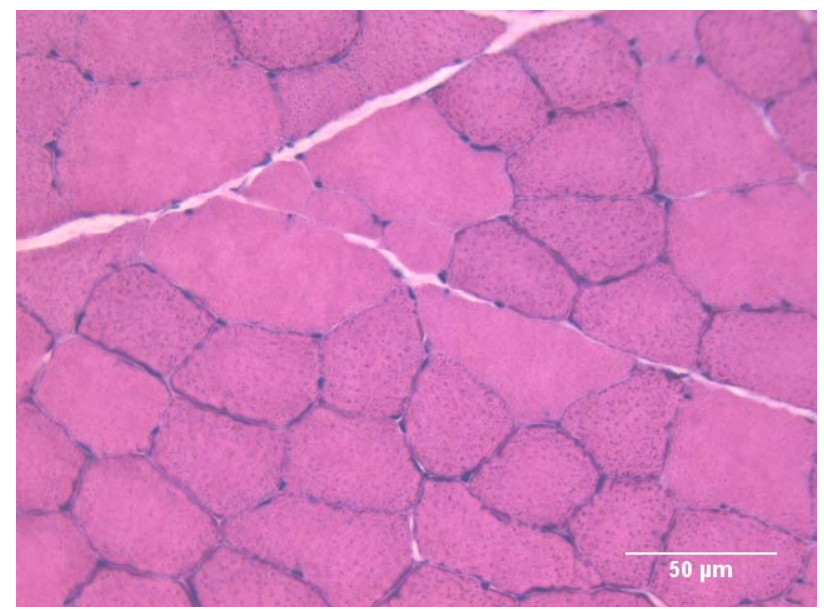


Figure 7

A) Young LTA

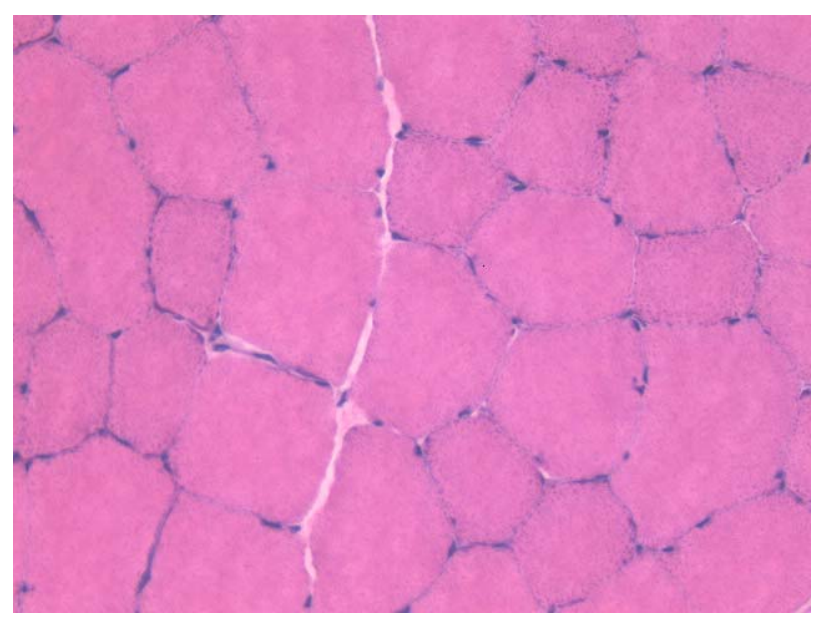

C) Old LTA

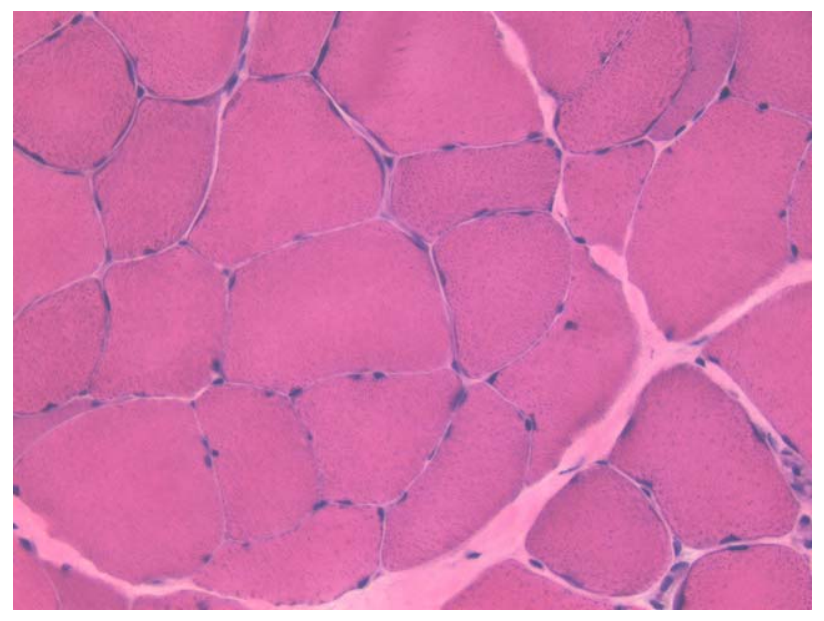

B) Young RTA

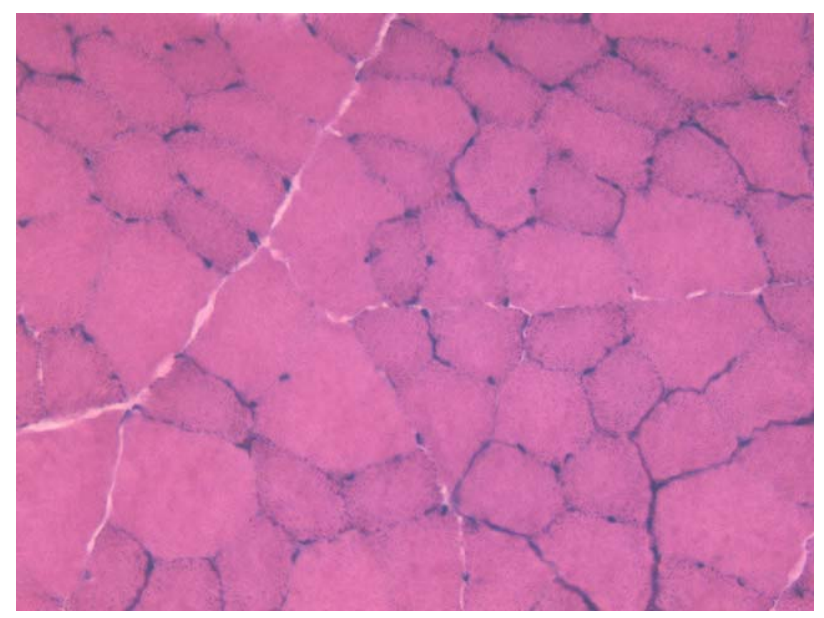

D) Old RTA

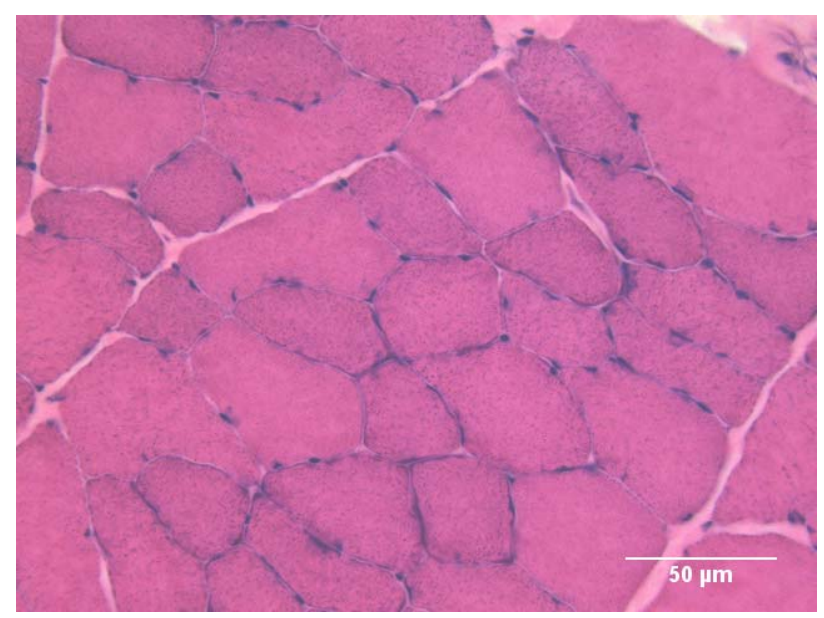


Figure 8a

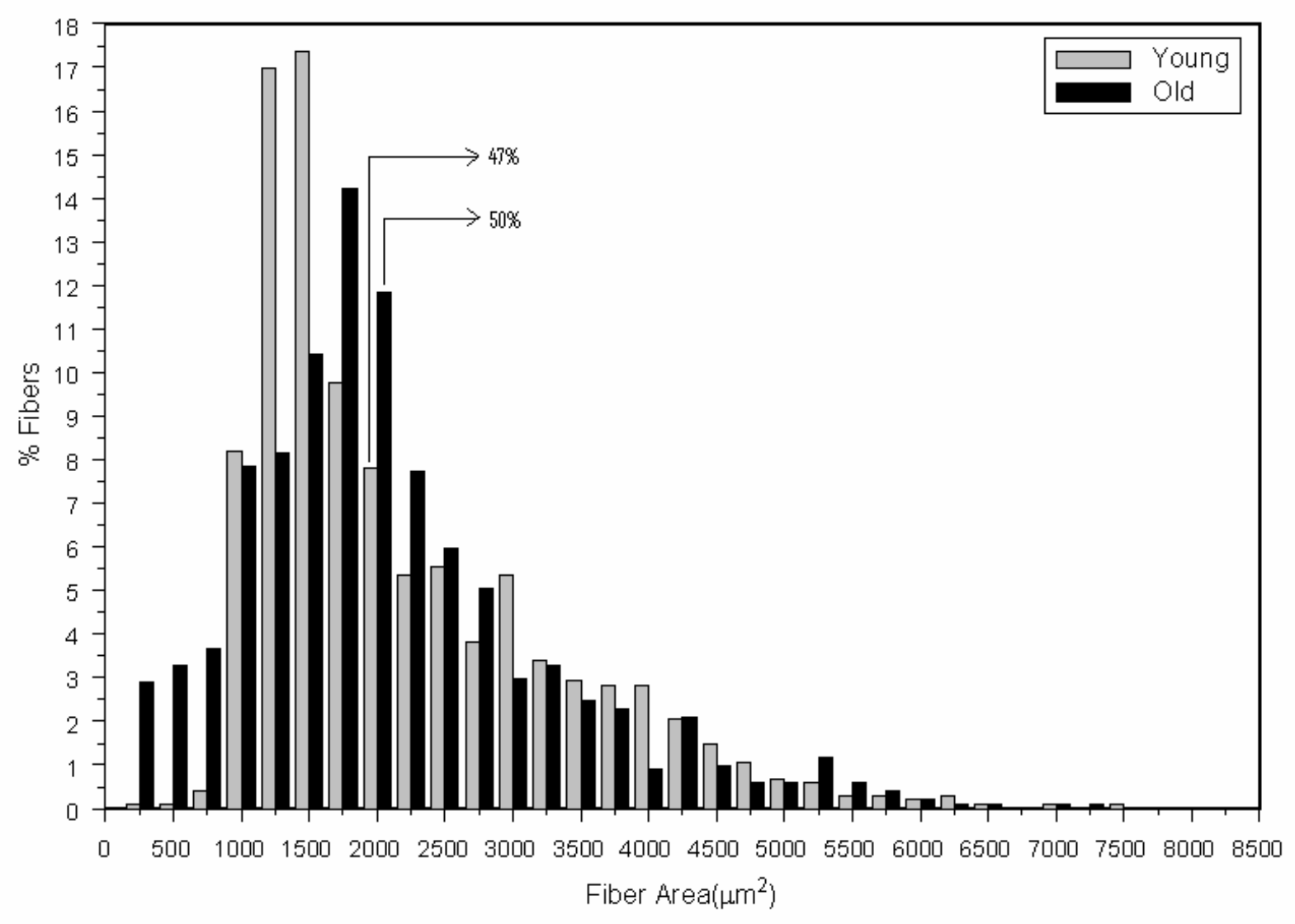


Figure 8b

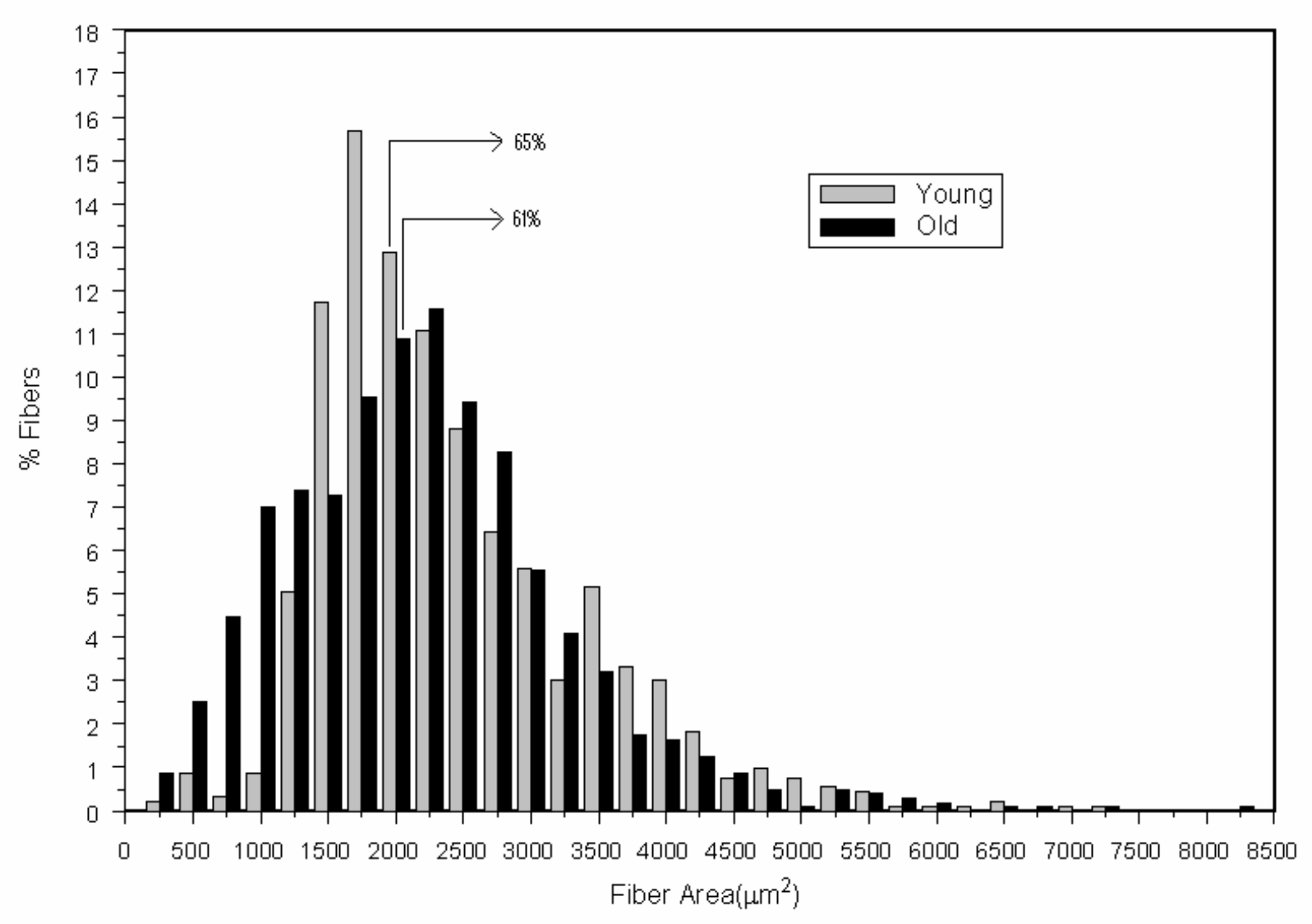


Figure 8c

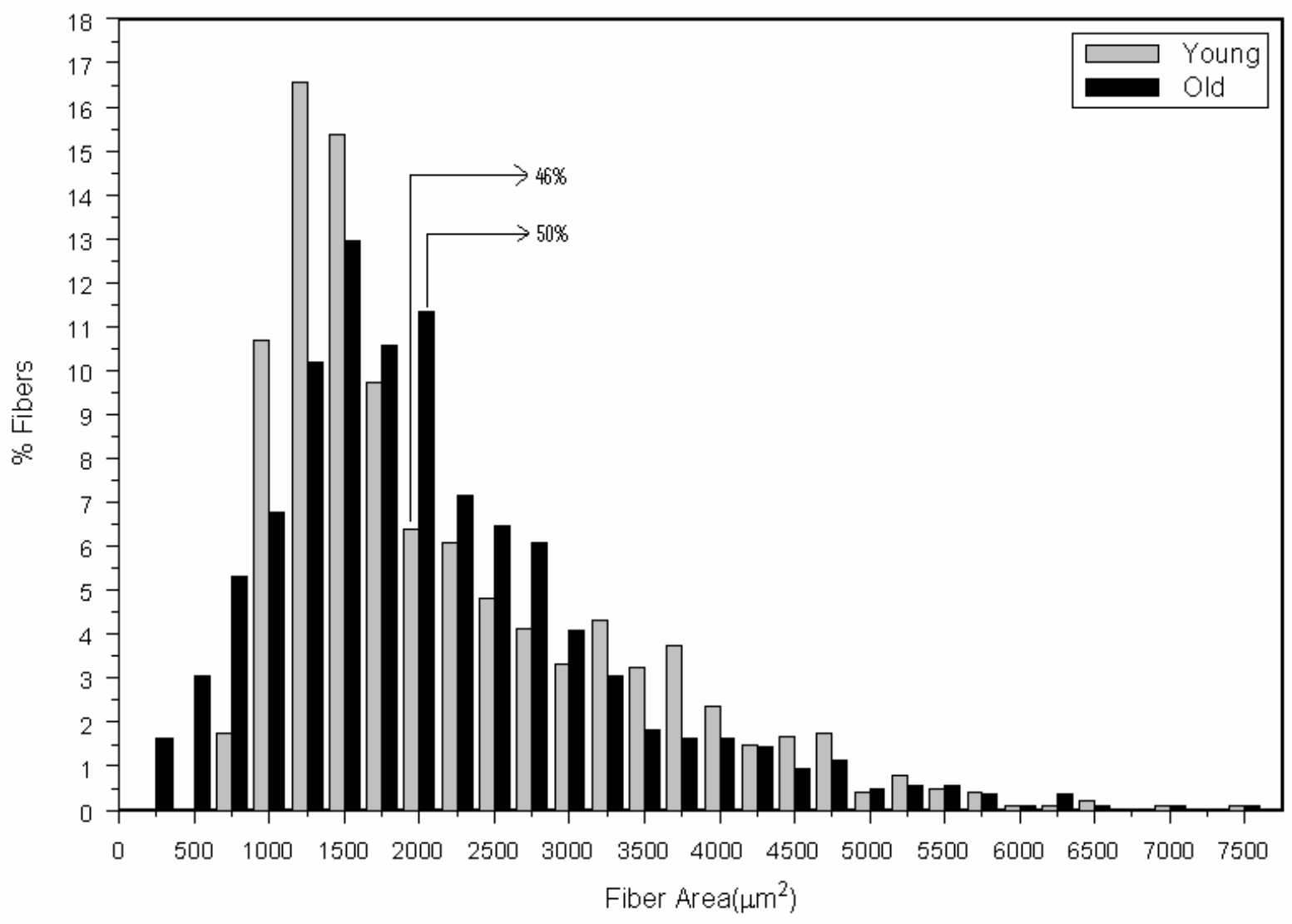


Figure 8d

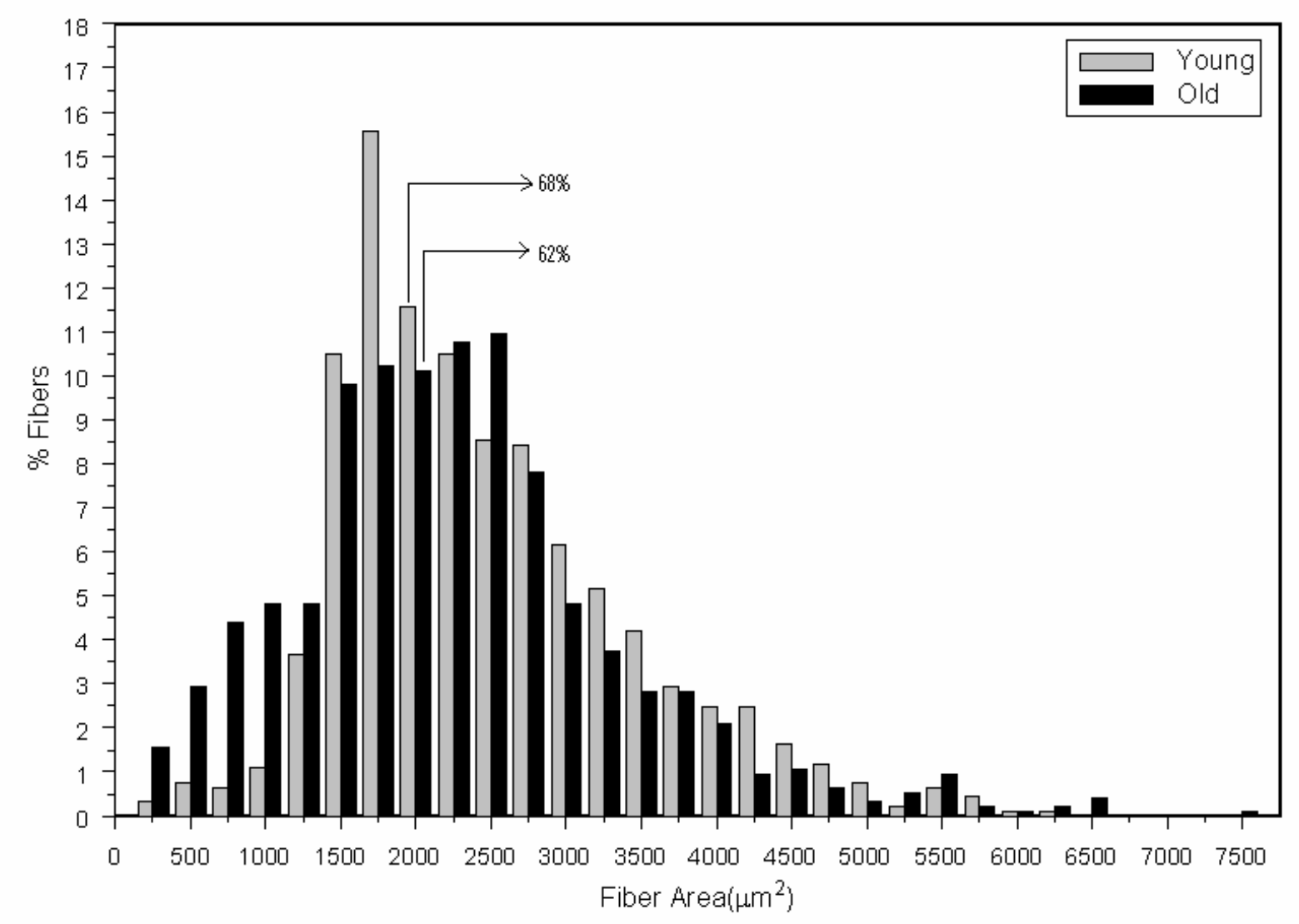


Myosin Heavy Chain Immunohistochemistry and Fiber Adaptation Following Chronic Stretch-Shortening Contraction Exposure: Influence of Glutathione Depletion and Age

\title{
Brent A. Baker ${ }^{1}$, Melinda S. Hollander ${ }^{1}$, Michael L. Kashon ${ }^{1}$, Robert G. Cutlip ${ }^{1}$
}

\author{
${ }^{1}$ National Institute for Occupational Safety and Health (NIOSH), Health Effects \\ Laboratory Division, Morgantown, WV, 26506, USA. \\ Address correspondence to: \\ Robert G. Cutlip, Ph.D. \\ National Institute for Occupational Safety and Health \\ Health Effects Laboratory Division \\ 1095 Don Nehlen Drive \\ $\mathrm{M} / \mathrm{S} 2027$ \\ Morgantown, WV 26505
}

Fax: (304) 285-6265; Tel: (304) 285-5968; E-mail: rgc8@cdc.gov

\section{Running head: Myosin Heavy Chain IHC Following Chronic Stretch-Shortening Contraction Exposure}




\section{ABSTRACT}

Background: The extent and contribution of myosin heavy chain plasticity in the adaptive response of skeletal muscle undergoing glutathione modulation following repetitive, highintensity mechanical loading is not known. Aim: The purpose of this research was to determine the influence dietary supplementation with a glutathione antagonist L-Buthionine Sulfoximine (BSO) has on the adaptability of skeletal muscle during chronic high-intensity mechanical loading via stretch-shortening contractions in young and old rodents. Research design and methods: Left dorsiflexor muscles of young (12 weeks, $\mathrm{N}=22$ ) and old (30 months, $\mathrm{N}=22$ ), vehicle- and BSO-treated rodents were exposed 3 times per week for 4.5-weeks to a protocol of 80 maximal SSCs per exposure in vivo. Myofiber plasticity was characterized by myosin heavy chain $\left(\mathrm{MHC}_{\text {slow }}{ }^{+}, \mathrm{MHC}_{\text {fast }}{ }^{+}\right.$, or $\left.\mathrm{MHC}_{\mathrm{dev}}{ }^{+}\right)$response by immunohistochemical analyses, as well as by changes in muscle cross-sectional area distribution following the exposure period. Results: $\mathrm{MHC}_{\text {slow }}{ }^{+}$content was increased in the old rodents, irrespective of treatment or exposure. Conversely, $\mathrm{MHC}_{\text {fast }}{ }^{+}$content was increased in the young rodents, regardless of treatment of exposure. $\mathrm{MHC}_{\mathrm{dev}}{ }^{+}$content was not changed in the current study. Shifts to larger fiber crosssectional areas were apparent for all exposed groups. Conclusions: $\mathrm{MHC}_{\mathrm{dev}}{ }^{+}$is not expressed following chronic mechanical loading. Aging skeletal muscle does retain some capacity for adaptation; however aging apparently diminishes the adaptive response following chronic SSC exposure. Evidently, a population of small myofibers does exist that do not undergo regeneration in aged skeletal muscle, perhaps accounting for the disparity observed between morphological adaptation and functional performance.

Keywords: Aging, Glutathione, Stretch-Shortening Contractions, Myosin Heavy Chain, Muscle Hypertrophy

\section{Publications Disclaimers:}

"The findings and conclusions in this report are those of the author(s) and do not necessarily represent the views of the National Institute for Occupational Safety and Health." 


\section{INTRODUCTION}

\section{Paragraph 1}

Injury to skeletal muscle has been investigated and primarily induced using an acute exposure to demanding or unfamiliar physical activity (Smith et al., 1997, Baker et al., 2006a, Baker et al., 2006b, Armstrong, 1990, Armstrong et al., 1983). Chronic physical exposure has been less successful in inducing overt skeletal muscle injury distinguished by its histopathology, however chronic exposure may lead to skeletal muscle mal-adaptation and dysfunction characterized and influenced by functional loss, pain, inflammation, fibrosis, and/or oxidative stress mismanagement (Sjogaard and Sogaard, 1998, Cutlip et al., 2006, Stauber, 2004, McArdle et al., 2002). More specifically, mechanical loading, via stretch-shortening contractions (SSCs), can result in adaptation that is represented by a shift in contractile, physiological, and morphological properties in young populations (Cutlip et al., 2006). This adaptive response appears to occur in aged populations as well, but it is delayed temporally and to a diminished extent (Brooks et al., 2001) (Baker et al. In review 2007a). Previously, we have shown that chronic SSC exposure does not produce myofiber degeneration, yet a population of small myofibers $\left(\leq 1,000 \mu \mathrm{m}^{2}\right)$ exist even in unperturbed rodent skeletal muscle and this is independent of age (Cutlip et al., 2006) (Baker et al. In preparation 2007b). Following chronic SSC exposure, this population of fibers is decreased in young rodents, but the reduction of this population of fibers in old rodents is not as noticeable. Examining if this population of myofibers contributes to the adaptive response may increase our understanding as to how aged skeletal muscle is able to respond to chronic mechanical loading.

\section{Paragraph 2}


Myosin heavy chain (MHC) expression is modulated in young and aged populations in response to altered neural and functional demands (Roy et al., 1997, Alway et al., 1995). Consequently, chronic SSC exposure may lead to changes in MHC expression and distribution with age. Following injury, electrical stimulation, and/or mechanical stretch to tissue, the developmental MHC isoform is re-expressed, and is an important factor in the regenerative state of skeletal muscle (Lieber et al., 1994, Alway et al., 1995, Smith et al., 1999) (Baker et al. In review 2007a). Yet the role this MHC isoform and others have on chronic SSC-induced muscle hypertrophy are less clearly understood.

\section{Paragraph 3}

Oxidative stress resulting from increased free radical production following physical exposure has been suggested to lead to negative changes in functional (McArdle et al., 2001) and cellular responses (McBride, 1998, McArdle et al., 1999) which is exacerbated in aged skeletal muscle (Zerba et al., 1990). Additionally, a recent study from our lab has shown that depletion of total glutathione levels in the target muscle can be achieved with L-Buthionine Sulfoximine (BSO), a glutathione antagonist, irrespective of age (Hollander et al., In preparation 2007). Additionally, BSO treatment increased measures of hydrogen peroxide in both young and old rodents (indicating increased oxidative stress), yet this increase in hydrogen peroxide was prevented with chronic, repetitive SSC exposure in young rodents only. Also chronic SSC exposure increased indices of lipid peroxidation and cell death in aged rodents only, but had no effect on hydrogen peroxide, irrespective of age. More specifically reactive oxygen species (ROS) may be a mechanism contributing to the injurious response observed in skeletal muscle following resistance training/mechanical loading (Uchiyama et al., 2006). Oxidative stress (or the balance 
in the host environment between the pro- and antioxidant status) may be a critical stimulus for skeletal muscle adaptation (Urso and Clarkson, 2003, McArdle et al., 2002). Without myofiber degeneration present, it is intriguing to postulate that modulating the oxidative stress profile may influence the extent to which individual MHC isoforms impact skeletal muscle hypertrophy.

\section{Paragraph 4}

Skeletal muscle represents the largest pool of total glutathione in the body, and glutathione is the most important non-protein thiol source that maintains most cells in a reduced environment (Meister, 1991). Since glutathione is a major antioxidant in the cell and serves various functions such as directly acting as a reducing agent, serving as a substrate for glutathione peroxidase (and others), and recycling radicals generated from other antioxidant molecules, the adaptive responses ensuing from oxidative stress modulation may be ascertained. Glutathione depletion can be accomplished using BSO, which is an irreversible inhibitor of $\gamma$-glutamylcysteine synthase (GCS) (Meister, 1991). BSO has been documented both physiologically and biochemically to chronically deplete levels of glutathione, and these rodents would be expected to be more vulnerable to mechanical loading-induced elevations in oxidative stress (Martensson, 1989, Leeuwenburgh and Ji, 1995) (Hollander et al., In preparation 2007).

\section{Paragraph 5}

Thus, the purpose of this study is to examine the influence glutathione depletion has on the adaptability of skeletal muscle MHCs and on muscle morphology with aging following chronic SSC exposure. Skeletal muscle adaptation was assessed by monitoring the changes in isometric and dynamic performance during the chronic exposure period, as well as cellular changes after 
completion of the SSC protocol that are detailed fully elsewhere (Baker et al., In preparation 2007b). Since developmental myosin expression has been coupled with the regenerative state following muscle injury, and previous results indicate that no myofiber degeneration was present following chronic SSC-induced adaptation in this model (Cutlip et al., 2006); we hypothesize that muscles undergoing glutathione depletion and chronic SSC exposure will not express developmental myosin due to decreased muscle regeneration. Further, the population of small myofibers that exist in old rodents following SSC exposure will not express developmental myosin, because these fibers contribute to age-related mal-adaptation.

\section{METHODS}

\section{Paragraph 6}

Animal handling: Male Fischer Brown Norway Hybrid rats (F344 x BN F1, N = 44) were obtained from the National Institutes on Aging colony. Young adult $(\mathrm{N}=22 ; 276 \pm 26$ grams, 12 weeks of age $)$ and old $(\mathrm{N}=22 ; 567 \pm 39$ grams, 30 months $)$ rats were housed in an AAALAC accredited animal quarters. After one week of acclimatization young and old rats were randomly divided into L-Buthionine Sulfoximine (BSO-treated, $(n=6)$ ), regular drinking water (vehicle, VEH-treated $(n=6))$, or cage-control groups $(\mathrm{CON}(\mathrm{n}=4))$, and all animals were subjected to a standardized experimental protocol approved by the NIOSH Animal Care and Use Committee. Temperature and light/dark cycle (dark cycle from 7:00 AM to 7:00 PM) were held constant for all animals; food and water were provided ad libitum for all groups, while water consumption was monitored for the experimental groups.

\section{Paragraph 7}


Glutathione Depletion: Rats randomized to the BSO-treated group received $300 \mathrm{ml}$ of water weekly supplemented with $10 \mathrm{mM}$ BSO in their drinking water, and water consumption was monitored weekly for all experimental groups. No differences were noted in water consumption during and after the exposure period with respect to age or treatment groups. The supplemented dose of BSO used in the current study was determined from a dose-response study in which total glutathione was significantly depleted in the tibialis anterior (TA) at the $10 \mathrm{mM}$ BSO concentration to approximately $65 \%$ of control levels (unpublished data).

\section{Paragraph 8}

Experimental setup: The dorsiflexor muscles were tested on a custom-built rodent dynamometer (Cutlip et al., 1997a). The experimental setup has been described in detail previously (Cutlip et al., 2004). Briefly, the dynamometer provides precise control over the muscle length and muscle force output parameters to be studied. The software acquired and stored position, force, and velocity data in real-time as described elsewhere (Cutlip et al., 2004). Rats were anesthetized with $2 \%$ isoflurane gas using a small animal anesthetic system (Surgivet Anesco Inc., Waukesha, WI, USA). Isoflurane was chosen because it has no effect on in vivo force production (Ingalls et al., 1996). After anesthesia, each rat was placed supine on the heated $\mathrm{x}-\mathrm{y}$ positioning table of the rodent dynamometer, with an anesthetic mask placed over its nose and mouth. The knee was secured in flexion (at $90^{\circ}$ ) with a knee holder. The left foot was secured in the load cell fixture using a custom-built foot holder with the ankle axis (assumed to be between the medial and lateral malleoli) aligned with the axis of rotation of the load cell fixture. Each animal was monitored during the protocol to ensure proper anesthetic depth and body temperature. 


\section{Paragraph 9}

Functional testing: As with the experimental set-up, the functional testing has been described in detail (Cutlip et al., 2004). Briefly, the joint position of the animal was defined by the angle between the tibia and the plantar surface of the foot. The angular position of the load cell fixture corresponded with the angular position of the ankle. The force produced by the dorsiflexor muscles was measured at the interface of the aluminum sleeve and the dorsum of the foot. Platinum stimulating electrodes (Grass Medical Instruments, Quincy MA, USA) were placed subcutaneously each exposure session to span the peroneal nerve. Activation of the electrical stimulator resulted in muscle contraction of the dorsiflexor muscle group. To reduce the effect of excitation-contraction fatigue, all electrical stimulation times were kept to a minimum with 2minutes (min) of recovery time between stimulations (Ingalls et al., 1998).

\section{Paragraph 10}

SSC protocol: Recently, the SSC protocol implemented in the current study has been described in detail (Cutlip et al., 2006). Briefly, young and old experimental groups were exposed to 8 sets of 10 repetitions of SSCs with 2-min intervals between each set. Within each set, there was a 2seconds (s) rest between each stretch-shortening contraction. For each repetition, the dorsiflexor muscles were fully activated for $100 \mathrm{~ms}$ duration via the electrical stimulator, and the eccentric contraction phase was initiated with a $60 \mathrm{deg} / \mathrm{s}$ movement velocity of the load cell fixture over the prescribed range of motion of 90-140 deg ankle angle. The load cell fixture was then immediately returned in the concentric phase at $60 \mathrm{deg} / \mathrm{s}$ to the starting position of $90 \mathrm{deg}$ ankle angle. The dorsiflexor muscles were then deactivated $300 \mathrm{~ms}$ later. Total stimulation time per repetition was $2.06 \mathrm{~s}$. 


\section{Paragraph 11}

\section{Isometric and Dynamic Force Tests:}

Testing for isometric and single SSCs have been detailed in length previously (Cutlip et al., 2004). Briefly, an isometric and a single SSC were measured on the dorsi-flexor muscle group preceding and immediately following the SSC protocol for each exposure period.

\section{Paragraph 12}

Chronic Exposure: The SSC protocol and performance tests were administered three times per week for a total of 14 exposures over a four and a half week period.

\section{Paragraph 13}

Muscle Processing: Twenty-four hours after the final exposure, rats were weighed, anesthetized with sodium pentobarbital (ip, $10 \mathrm{mg} / 100 \mathrm{~g}$ body weight) and exsanguinated. The left (exposed LTA) and right (contra-lateral control - RTA) tibialis anterior muscles were dissected, weighed, and divided into 5 equal-distant "zones" ("Zone 1" most proximal - "Zone 5" most distal), and mounted onto cork board with O.C.T. embedding compound (VWR, West Chester, Pennsylvania), frozen in liquid nitrogen-cooled isopentane, and stored at $-80^{\circ} \mathrm{C}$ until sectioned for histology. Collection and preparation of samples was standardized, $3 \mathrm{~min}$ at room temperature was allowed for all procedures prior to freeze fixation in isopentane cooled in liquid nitrogen. "Zone 3" was selected to obtain the maximum tissue sample; this corresponds to the TA mid-belly.

\section{Paragraph 14}


Histology and Immunohistochemistry (IHC): Frozen, serial transverse sections were cut at 12 micron $(\mu \mathrm{m})$ thickness from "Zone 3", thaw mounted onto pre-coated microscope slides, air dried, and stored at $-80^{\circ} \mathrm{C}$ until processing. Immunohistochemistry for slow-type myosin heavy chain $\left(\mathrm{MHC}_{\text {slow }}{ }^{+}\right)$, fast-type myosin heavy chain $\left(\mathrm{MHC}_{\text {fast }}{ }^{+}\right)$, and developmental-type myosin heavy chain $\left(\mathrm{MHC}_{\mathrm{dev}}{ }^{+}\right.$) was performed using serial sections (one slide) from the RTA and LTA of each group. All antibodies were diluted in 0.01 M phosphate buffered saline (PBS, pH 7.4) containing 4\% Triton-X 100 (PBS-TX). The MHC primary antibodies were obtained from Nova Castra Labs (Newcastle, UK), and the fluorescently tagged secondary antibody (Cy2) and normal donkey serum for blocking were obtained from Jackson Immuno-Research Laboratories Inc. (West Grove, PA).

\section{Paragraph 15}

MHC IHC: Frozen sections used for $\mathrm{MHC}$ immunolabeling that were stored at $-80^{\circ} \mathrm{C}$ were brought to room temperature and rinsed in PBS + 0.1M Glycine for $5 \mathrm{~min}$. All slides were incubated in blocking buffer (5\% normal donkey serum diluted in PBS + Triton X-100) at room temperature for $2 \mathrm{~h}$. Slides were then incubated in either mouse anti- $\mathrm{MHC}_{\text {slow }}{ }^{+},-\mathrm{MHC}_{\text {fast }}{ }^{+}$, or $\mathrm{MHC}_{\mathrm{dev}}{ }^{+}$antibody diluted in blocking buffer at $\left(1: 50,1: 25\right.$, or $1: 20$, respectively) for $1 \mathrm{~h}$ at $4{ }^{\circ} \mathrm{C}$. Following incubation, sections were rinsed in PBS and incubated for $1 \mathrm{~h}$ at RT in Cy2 labeled donkey anti-mouse IgG (1:200). Slides were then rinsed, incubated in DAPI to stain nuclei (1:1000, Sigma, St. Louis, MO), coverslipped using Prolong Gold antifade reagent (Molecular Probes), and allowed to dry in a cool, dark area. A number of slides were processed in the absence of the primary antibody as controls for non-specific binding. 


\section{Paragraph 16}

MHC Localization and Quantification: Photomicrographs were captured and saved using an Olympus Photomicroscope and Simple PCI Image Analysis Software. Quantification of positively immunolabeled MHC sections was performed using 1 section per slide from the contra-lateral control RTA and exposed LTA muscles from each group. Digital color images were analyzed using standard quantitative morphometric methods (Weibel, 1972, Weibel, 1974, Weibel, 1975, Underwood, 1970) to measure the volume density (\% tissue fraction) of $\mathrm{MHC}_{\text {slow }}{ }^{+}, \mathrm{MHC}_{\text {fast }}{ }^{+}$, or $\mathrm{MHC}_{\mathrm{dev}}{ }^{+}$positively labeled myofibers. Briefly, each of the MHC sections was quantified independently, as one section per slide was analyzed from each animal per group. A stage micrometer was used to identify the mid-point of the section. Point and intercept counts using a 121-point / 11-line overlay graticule (12.5 mm square with 100 divisions) at $40 \mathrm{X}$ magnification were taken at 5 equally spaced points across the section. This process was repeated $2 \mathrm{~mm}$ on either side of the mid-point of the section for a total of 1210 points per section. Volume density (\% tissue fraction) was computed from the percentage of points over the tissue section to points over each of the $\mathrm{MHC}_{\text {slow }}{ }^{+}, \mathrm{MHC}_{\text {fast }}{ }^{+}$, or $\mathrm{MHC}_{\mathrm{dev}}{ }^{+}$labeled myofibers. MHC data are reported as mean \pm SE and analyzed as described below.

\section{Paragraph 17}

Fiber cross-sectional area: For muscle fiber cross-sectional area (CSA) analysis, ten nonoverlapping digital images were obtained from hematoxylin and eosin-stained muscle sections at a 40X magnification and, subsequently, were analyzed for TA fiber CSA $(\mu \mathrm{m} 2)$ with Optimas Image Analysis Software. Each fiber was traced with a handheld mouse, and the number of pixels traced was calibrated to a defined area in square micrometers. Approximately 200 fibers 
were traced per sample as previously described (Cutlip et al., 2006) and cumulative frequency histograms were generated for comparisons of myofiber CSA distributions.

\section{DATA ANALYSIS}

\section{Paragraph 18}

MHC IHC: MHC volume density was analyzed using three-way mixed model (treatment $\mathrm{x}$ age $\mathrm{x}$ limb) ANOVA with the animal as the random factor accounting for measures in both limbs. Differences with a probability of $p<0.05$ were considered significant. All data were analyzed using JMP v. 5.1 (SAS Institute Inc., Cary, NC).

\section{$\underline{\text { RESULTS }}$}

\section{Paragraph 19}

Performance Data: The complete set of functional data for all groups illustrating the force and work response at the onset and following exposure are reported elsewhere (Baker et al., In preparation 2007b). These data demonstrate that young rodents, regardless of treatment, generally increased muscle function following exposure. However old rodents, regardless of treatment, were unsuccessful in increasing muscle function after the exposure period.

\section{Paragraph 20}

Body and TA muscle wet-weights weights: The complete data set for the average body weights and muscle wet-weights for the young and old rodents are also reported elsewhere (Baker et al., In preparation 2007b). 


\section{Paragraph 21}

Fiber Cross-sectional area: Muscle fiber cross-sectional areas (CSA) have recently been reported in their entirety (Baker et al., In preparation 2007b). Cumulative frequency histograms clearly depict a population of small myofibers $(\leq 1,000 \mu \mathrm{m})$ that comprise $\sim 20 \%$ of the total fiber population in old rodents, regardless of treatment (Figures 1a-1c). This population exists and is equally distributed in both limbs in the old, CON rodents (Figure 1c), and still persists following exposure, however exposure tends to decrease the percentage of these fibers. Cumulative frequency histograms illustrate that exposed muscles from young rodents exhibited marked hypertrophy, regardless of treatment (Figures 2a-2c). Cumulative frequency histograms from old rodents reveal that while muscle hypertrophy was present in the VEH- and BSOtreated, aging diminished this effect (Figures 1a, 1b).

\section{Paragraph 22}

MHC IHC: Age influenced the volume density of both the $\mathrm{MHC}_{\text {slow }}{ }^{+}$labeled myofibers and $\mathrm{MHC}_{\text {fast }}{ }^{+}$labeled myofibers. Old rodents had increased $\mathrm{MHC}_{\text {slow }}{ }^{+}$labeling compared to young counterparts, irrespective of treatment or $\operatorname{limb}(\mathrm{p}<0.05$, Figure $3(\mathrm{~A}-\mathrm{F})$ and Figure 4$) . \mathrm{MHC}_{\text {fast }}{ }^{+}$ labeled myofibers were increased in young rodents compared to old rodents, regardless of treatment or limb $(\mathrm{p}<0.05$, Figure $5(\mathrm{~A}-\mathrm{F})$ and Figure 6). All other comparisons were not significant.

\section{DISCUSSION}

\section{Paragraph 23}


BSO treatment resulted in a marked decrease in total glutathione levels in the TA muscle tissue (Hollander et al., In preparation 2007), and this is consistent with the literature (Leeuwenburgh et al., 1994). Even though muscle adaptation does occur following repetitive mechanical loading in young rodents (this response is diminished with aging), developmental MHC was not present at 4.5 weeks in either age group. We hypothesized this was due to the lack of degeneration incurred during the initial as well as subsequent exposure/s (Baker et al., In review 2007a, Baker et al, In preparation 2007b). Whether developmental MHC is an important stimulus or is expressed at earlier time points following chronic SSC exposure has not been reported, but we and others have reported this event at early time points in the overall adaptive response following acute exposure (Roy et al., 1997) (Baker et al., In review 2007a). Although insignificant, a lowlevel of developmental MHC labeling was present in both young and old rodent's limbs in the present study, and this is consistent with previous reports that there is a minimal expression of developmental MHC in naïve rodent muscle (Smith et al., 1999). Regardless of the void in the developmental MHC labeling profile, there still existed a sizeable population $(\sim 15$ in the exposed limbs and $\sim 20 \%$ in the cage control limbs) of small myofibers in muscles from old rodents that visibly do not undergo regeneration following exposure. Thus, the contribution of these myofibers to SSC-induced muscle hypertrophy appears not to be significant. An alternate explanation may be that this population of small fibers was the result of exposure-induced atrophy, and thus it would be plausible from our hypothesis that these myofibers may be contributing to age-related muscle mal-adaptation. Again, this does not appear to occur, since in the old, VEH- and BSO-treated rodents there was moderate hypertrophy of skeletal muscle fibers with respect to young counterparts, and this occurred without a noticeable decrease in performance (Baker et al., In preparation 2007b). Additionally, chronic SSC exposure of old 
rodents slightly decreased the percentage of this population of small myofibers. A lesser percentage of small fibers existed in the cage control limbs of young rodents $(\sim 10 \%)$ when compared with old counterparts $(\sim 20 \%)$ and exposure further decreased the percentage of small fibers in young rodents $(\sim 3 \%)$ and old rodents $(\sim 15 \%)$, regardless of treatment. This may provide a general explanation to the discrepancy that exists between the differential amount of adaptation between young and old populations. Since a considerable population of small fibers exist in old rodents even after chronic exposure, these small fibers constituting a remaining motor unit may be a limiting factor for performance as well as physiological adaptation. In contrast to old rodents, if at an earlier time-point during the 4.5 weeks of exposure the small myofiber population in the young rodents transiently expressed developmental myosin in response to an adaptive stimulus, then the decrease in the percentage of these fibers may impact the hypertrophy process in young rodents.

Paragraph 24: Avian models using stretch overload have successfully documented the generation of new fiber formation that accompanies the overall rapid and robust hypertrophic response (Alway et al., 1995, McCormick and Schultz, 1992). Underlying this mechanism of new fiber formation is the premise that the existence of this particular population of small fibers we observed would express some form of developmental myosin, since it has been shown that new fiber formation also is accompanied by an acute phase as well as long-lasting expression of this MHC (McCormick and Schultz, 1992, Alway et al., 1995). However, in our 4.5 week SSC exposure model, the impact this mechanism's contribution has on this population of small myofibers is not significant. This is consistent with the contention that fiber number remains constant with other models of mechanical loading as well (Roy et al., 1997). Nonetheless, we 
have shown that young rodents adapt with marked hypertrophy to chronic SSC exposure regardless of how the glutathione system is modulated. Given the correct stimulus (as applied in the present study), it is also apparent that there may be some residual capacity for this to occur in aging skeletal muscle as well, since we did see muscle hypertrophy in old, VEH- and BSOtreated rodents. This may be achieved by specifically and selectively conserving glutathione in metabolically active tissue and allowing for maintenance of increased oxidative stress (Leeuwenburgh and Ji, 1995) or by compensatory mechanisms following glutathione depletion. Thus, an alternate mechanism/s owing to the existence of both the population of small myofibers and those fibers undergoing hypertrophy may exist.

Paragraph 25: Aging influenced both the slow and fast $\mathrm{MHC}$ distribution. We observed an increase in the slow MHC in old rodents compared to young rodents, while there was an increase in proportion of fast MHC in the young rodents compared with old counterparts. This differential MHC distribution with aging is consistent with what has been reported previously in the literature (Alnaqeeb and Goldspink, 1987, Holloszy et al., 1991, Roy et al., 1997). Similarities in the volume density of the slow and fast $\mathrm{MHC}$ following exposure, indicates that 4.5 weeks of SSC exposure has little impact on fiber phenotype. Furthermore, modulation of the glutathione system did not influence the plasticity of the MHC profile.

Paragraph 26: From a qualitative perspective, the general observation that small myofibers expressed fast MHC whereas slow MHC was seldom expressed in small fibers is important, and indicates that this population of small myofibers in old rodents may be undergoing denervation. This is corroborated by reports demonstrating that the proportion of type II fibers decreases with 
age and may result from denervation with subsequent reinnervation (Holloszy et al., 1991, Pettigrew and Gardner, 1987). Recently, Krajnak and colleagues (Krajnak et al., 2006) have shown that satellite cells in old rodents express increased levels of the proapoptotic factor Bax compared with young counterparts, and this may be influential in the limiting recovery from mechanical exposure. It is attractive to postulate that these satellite cells expressing the proapoptotic Bax protein exist primarily in type II fibers and contribute to limiting age-related adaptation. Additionally, a recent study in our lab has illustrated that myofibers from aged rodents have diminished immunopositive developmental myosin labeling compared with young rodents at 5 day recovery when acutely exposed to the current adaptive SSC protocol (Baker et al., In review 2007). Together these data suggest that aged rodents not only preferentially are undergoing apoptosis of satellite cells, but also have a diminished regenerative capacity compared with young counterparts which may negatively regulate their adaptation to mechanical loading.

Paragraph 27: There are certain factors that limit the interpretations of our findings in the current study. First, with respect to fiber type distribution, our monoclonal antibody only detected fast MHC, as subtypes were not differentiated. Additionally, difficulties exist in drawing definitive conclusions as to the influence glutathione has on a more general adaptive response to exposure with aging, since there are obvious disparities that exist between modes of exposure. Also, there is a clear void in the literature regarding the role of glutathione as well as other antioxidants and their impact on skeletal muscle adaptation with mechanical loading and aging. 
Paragraph 28: These findings indicate that manipulating the glutathione system has no impact on MHC expression following chronic SSC exposure. Additionally, glutathione depletion does not adversely limit muscle hypertrophy, since both young and old rodents responded with muscle hypertrophy. Aging, however, does limit the response of skeletal muscle hypertrophy to mechanical loading. Thus, following chronic SSC exposure, developmental MHC is not expressed, yet muscular adaptation clearly results. That the temporal expression of developmental forms of myosin following an acute bout of this type of SSCs (modified to produce adaptation in the absence of myofiber degeneration) may be indicative of long-term adaptation is intriguing and must be examined in detail. 


\section{REFERENCES}

ALNAQEEB, M. A. \& GOLDSPINK, G. (1987) Changes in fibre type, number and diameter in developing and ageing skeletal muscle. J Anat, 153, 31-45.

ALWAY, S. E., DEGENS, H., KRISHNAMURTHY, G. \& SMITH, C. A. (2002) Potential role for Id myogenic repressors in apoptosis and attenuation of hypertrophy in muscles of aged rats. Am J Physiol Cell Physiol, 283, C66-76.

ARMSTRONG, R. B. (1990) Initial events in exercise-induced muscular injury. Med Sci Sports Exerc, 22, 429-35.

ARMSTRONG, R. B., OGILVIE, R. W. \& SCHWANE, J. A. (1983) Eccentric exercise-induced injury to rat skeletal muscle. J Appl Physiol, 54, 80-93.

BAKER, B. A., MERCER, R. R., GERONILLA, K. B., KASHON, M. L., MILLER, G. R. \& CUTLIP, R. G. (2006a) Stereological analysis of muscle morphology following exposure to repetitive stretch-shortening cycles in a rat model. Appl Physiol Nutr Metab, 31, 16779.

BAKER, B. A., RAO, K. M., MERCER, R. R., GERONILLA, K. B., KASHON, M. L., MILLER, G. R. \& CUTLIP, R. G. (2006b) Quantitative histology and MGF gene expression in rats following SSC exercise in vivo. Med Sci Sports Exerc, 38, 463-71.

BAKER, B. A., HOLLANDER, M. S., KASHON, M. L., \& CUTLIP, R. G.

(In review 2007a) Adaptive stretch-shortening contractions: diminished regenerative capacity with aging.

BAKER, B. A., HOLLANDER, M. S., MERCER, R. R., KASHON, M. L., \& CUTLIP, R. G. (In preparation 2007b) Glutathione Depletion and Age on Performance and Skeletal Muscle Morphology Following Chronic Stretch-Shortening Contraction Exposure.

CUTLIP, R. G., BAKER, B. A., GERONILLA, K. B., MERCER, R. R., KASHON, M. L., MILLER, G. R., MURLASITS, Z. S. \& ALWAY, S. E. (2006) Chronic exposure of stretch-shortening contractions results in skeletal muscle adaptation in young rats and maladaptation in old rats. Applied Physiology, Nutrition and Metabolism, 31, 573-587.

CUTLIP, R. G., GERONILLA, K. B., BAKER, B. A., KASHON, M. L., MILLER, G. R. \& SCHOPPER, A. W. (2004) Impact of muscle length during stretch-shortening contractions on real-time and temporal muscle performance measures in rats in vivo. $J$ Appl Physiol, 96, 507-16. 
CUTLIP, R. G., STAUBER, W. T., WILliSON, R. H., MCINTOSH, T. A. \& MEANS, K. H. (1997) Dynamometer for rat plantar flexor muscles in vivo. Medical \& Biological Engineering \& Computing, 35, 540-3.

HOLLANDER, M.S., (In preparation 2007)

HOLlOSZY, J. O., CHEN, M., CARTEE, G. D. \& YOUNG, J. C. (1991) Skeletal muscle atrophy in old rats: differential changes in the three fiber types. Mech Ageing Dev, 60, 199-213.

INGALLS, C. P., WARREN, G. L., LOWE, D. A., BOORSTEIN, D. B. \& ARMSTRONG, R. B. (1996) Differential effects of anesthetics on in vivo skeletal muscle contractile function in the mouse. Journal of Applied Physiology, 80, 332-40.

INGALlS, C. P., WARREN, G. L., WILliAMS, J. H., WARD, C. W. \& ARMSTRONG, R. B. (1998) E-C coupling failure in mouse EDL muscle after in vivo eccentric contractions. $J$ Appl Physiol, 85, 58-67.

KRAJNAK, K., WAUGH, S., MILLER, R., BAKER, B., GERONILLA, K., ALWAY, S. E. \& CUTLIP, R. G. (2006) Proapoptotic factor bax is increased in satellite cells in the tibialis anterior muscles of old rats. Muscle Nerve, 24, 720-730.

LEEUWENBURGH, C. \& JI, L. L. (1995) Glutathione Depletion in Rested and Exercised Mice: Biochemical Consequence and Adaptation. Arch Biochem Biophys, 316, 941-949.

LIEBER, R. L., SCHMITZ, M. C., MISHRA, D. K. \& FRIDEN, J. (1994) Contractile and cellular remodeling in rabbit skeletal muscle after cyclic eccentric contractions. $J$ Appl Physiol, 77, 1926-34.

MARTENSSON, J., MEISTER, A. (1989) Mitochondrial damage in muscle occurs after marked depletion of glutathione and is prevented by giving glutathione monoester. Proc Natl Acad Sci U S A, 86, 471-475.

MCARDLE, A., PATTWELL, D., VASILAKI, A., GRIFFITHS, R. D. \& JACKSON, M. J. (2001) Contractile activity-induced oxidative stress: cellular origin and adaptive responses. Am J Physiol Cell Physiol, 280, 621-627.

MCARDLE, A., VAN DER MEULEN, J. H., CATAPANO, M., SYMONS, M. C., FAULKNER, J. A. \& JACKSON, M. J. (1999) Free radical activity following contraction-induced injury to the extensor digitorum longus muscles of rats. Free Radic Biol Med, 26, 1085-91. 
MCARDLE, A., VASILAKI, A. \& JACKSON, M. J. (2002) Exercise and skeletal muscle ageing: cellular and molecular mechanisms. Ageing Research Reviews, 1, 79-93.

MCBRIDE, J. M., KRAEMER, W.J., TRIPLETT-MCBRIDE, T., SEBASTIANELLI, W. (1998) Effect of resistance exercise on free radical production. Med Sci Sports Exerc, 30, 67-72.

MCCORMICK, K. M. \& SCHULTZ, E. (1992) Mechanisms of nascent fiber formation during avian skeletal muscle hypertrophy. Dev Bio, 150, 319-334.

MEISTER, A. (1991) Glutathione deficiency produced by inhibition of its synthesis, and its reversal; applications in research and therapy. Pharmac. Ther., 51.

PETTIGREW, F. P. \& GARDNER, P. F. (1987) Changes in rat plantaris motor unit profiles with advanced age. Mech Ageing Dev, 40, 243-259.

ROY, R. R., TALMADGE, R. J., FOX, K., LEE, M., ISHIHARA, A. \& EDGERTON, V. R. (1997) Modulation of MHC isoforms in funtionally overloaded and exercised rat plantaris fibers. J Appl Physiol, 83, 280-290.

SJOGAARD, G. \& SOGAARD, K. (1998) Muscle injury in repetitive motion disorders. Clin Orthop, 21-31.

SMITH, H. K., PLYLEY, M. J., RODGERS, C. D. \& MCKEE, N. H. (1997) Skeletal muscle damage in the rat hindlimb following single or repeated daily bouts of downhill exercise. Int J Sports Med, 18, 94-100.

SMITH, H. K., PLYLEY, M. J., RODGERS, C. D. \& MCKEE, N. H. (1999) Expression of developmental myosin and morphological characteristics in adult rat skeletal muscle following exercise-induced injury. Eur J Physiol, 80, 84-91.

STAUBER, W. T. (2004) Factors involved in strain-induced injury in skeletal muscles and outcomes of prolonged exposures. J Electromyogr Kinesiol, 14, 61-70.

UCHIYAMA, S., TSUKAMOTO, H., YOSHIMURA, S. \& TAMAKI, T. (2006) Relationship between oxidative stress in muscle tissue and weight-lifting-induced muscle damage. Eur J Physiol, 452, 109-116.

UNDERWOOD, E. E. (1970) Quantatative Stereology, Reading, MA, Addison-Wesley Publishing Co.

URSO, M. L. \& CLARKSON, P. M. (2003) Oxidative stress, exercise, and antioxidant supplementation. Toxicology, 189, 41-54. 
WEIBEL, E. R. (1972) The value of stereology in analysing structure and function of cells and organs. J Microsc, 95, 3-13.

WEIBEL, E. R. (1974) Selection of the best method in stereology. J Microsc, 100, 261-9.

WEIBEL, E. R. (1975) Quantitation in morphology: possibilities and limits. Beitr Pathol, 155, 117.

ZERBA, E., KOMOROWSKI, T. E. \& FAULKNER, J. A. (1990) Free radical injury to skeletal muscles of young, adult, and old mice. Am J Physiol, 258, C429-35. 


\section{FIGURE LEGENDS}

Figure 1: Muscle fiber cumulative frequency histograms for old VEH- or BSO-treated and $\mathrm{CON}$ rodents following the 4.5 week chronic exposure period. In the old $\mathrm{CON}$ rodents note the existence of a population of small myofibers $\left(\leq 1,000 \mu \mathrm{m}^{2}\right)$ accounting for $\sim 18-20 \%$ of the total number of fibers sampled, regardless of limb. Exposure leads to a slight decrease in the percentage of this population of small fibers in old, VEH- and BSO-treated rodents.

Figure 2: Muscle fiber cumulative frequency histograms for young VEH- or BSO-treated and CON rodents following the 4.5 week chronic exposure period. In the young $\mathrm{CON}$ rodents note the existence of a population of small myofibers $\left(\leq 1,000 \mu \mathrm{m}^{2}\right)$ accounting for $\sim 8-10 \%$ of the fibers sampled, regardless of limb. Exposure leads to a decrease in the percentage of this population of small fibers in young rodents, regardless of treatment.

Figure 3: Representative photomicrographs illustrating $\mathrm{MHC}_{\text {slow }}{ }^{+}$immunopositive cells in TA muscle's of young (A, C, E) and old (B, D, F) rodents. Images A and B are from VEH-treated rodents, images $\mathrm{C}$ and $\mathrm{D}$ are from $\mathrm{BSO}$-treated rodents, and images $\mathrm{E}$ and $\mathrm{F}$ are from $\mathrm{CON}$ rodents. All micrographs shown are captured at 40X magnification.

Figure 4: Volume density was computed from the percentage of points over the tissue section to points over each of the $\mathrm{MHC}_{\text {slow }}{ }^{+}$labeled myofibers. $* *$ Significant effect of aging $(\mathrm{p}<0.05)$. MHC data are reported as mean values +/- standard error. 
Figure 5: Representative photomicrographs illustrating $\mathrm{MHC}_{\text {fast }}{ }^{+}$immunopositive cells in TA muscle's of young (A, C, E) and old (B, D, F) rodents. Images A and B are from VEH-treated rodents, images $\mathrm{C}$ and $\mathrm{D}$ are from $\mathrm{BSO}$-treated rodents, and images $\mathrm{E}$ and $\mathrm{F}$ are from $\mathrm{CON}$ rodents. All micrographs shown are captured at 40X magnification.

Figure 6: Volume density was computed from the percentage of points over the tissue section to points over $\mathrm{MHC}_{\text {fast }}{ }^{+}$labeled myofibers. $* *$ Significant effect of aging $(\mathrm{p}<0.05)$. MHC data are reported as mean values $+/$ - standard error. 
Figure 1a

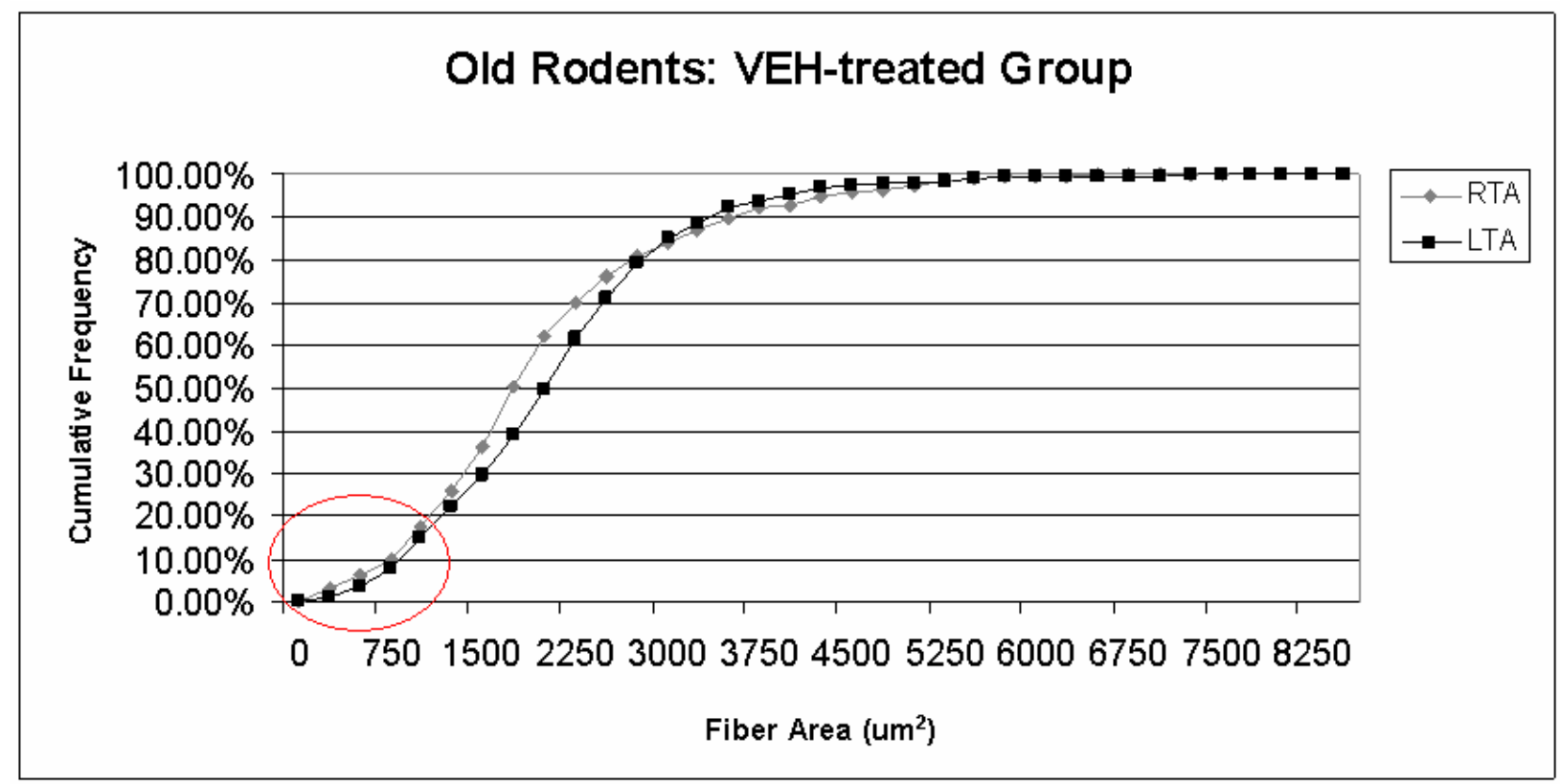

Figure 1b

\section{Old Rodents: BSO-treated Group}

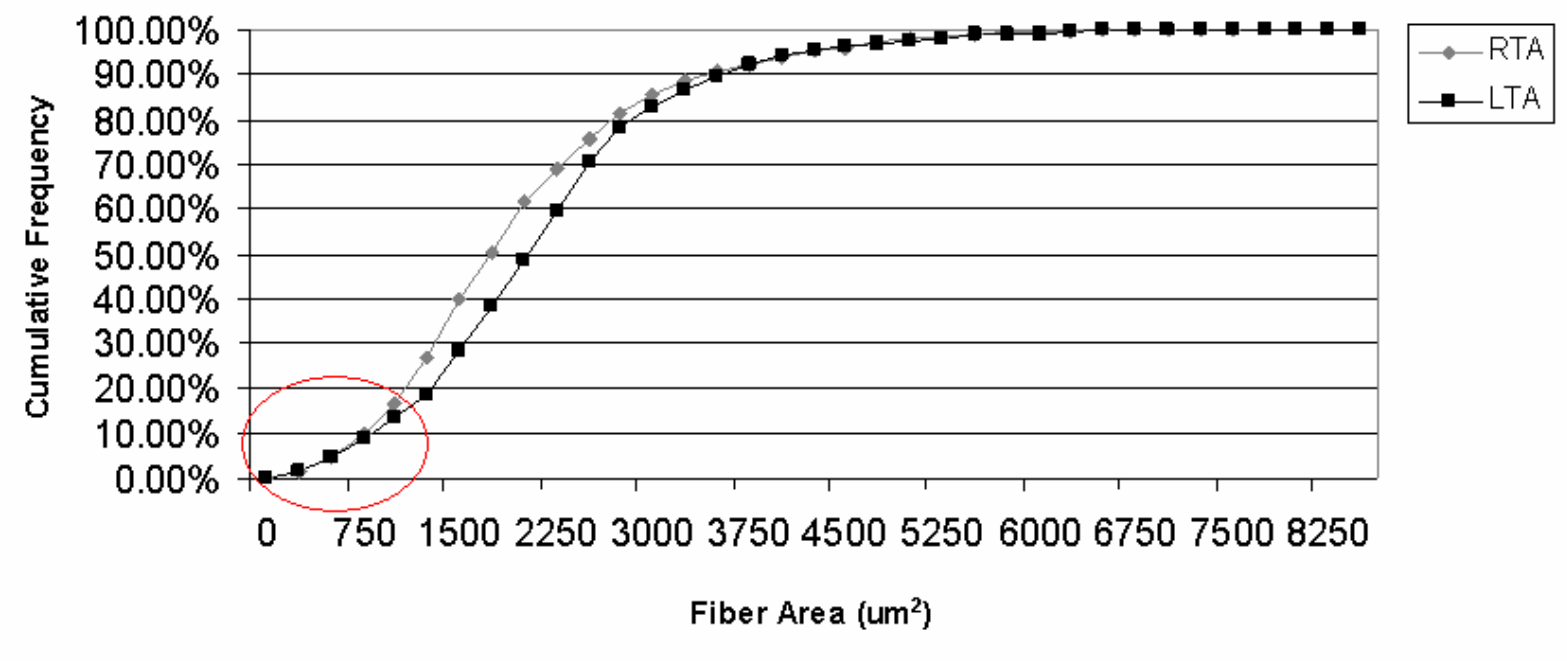


Figure 1c

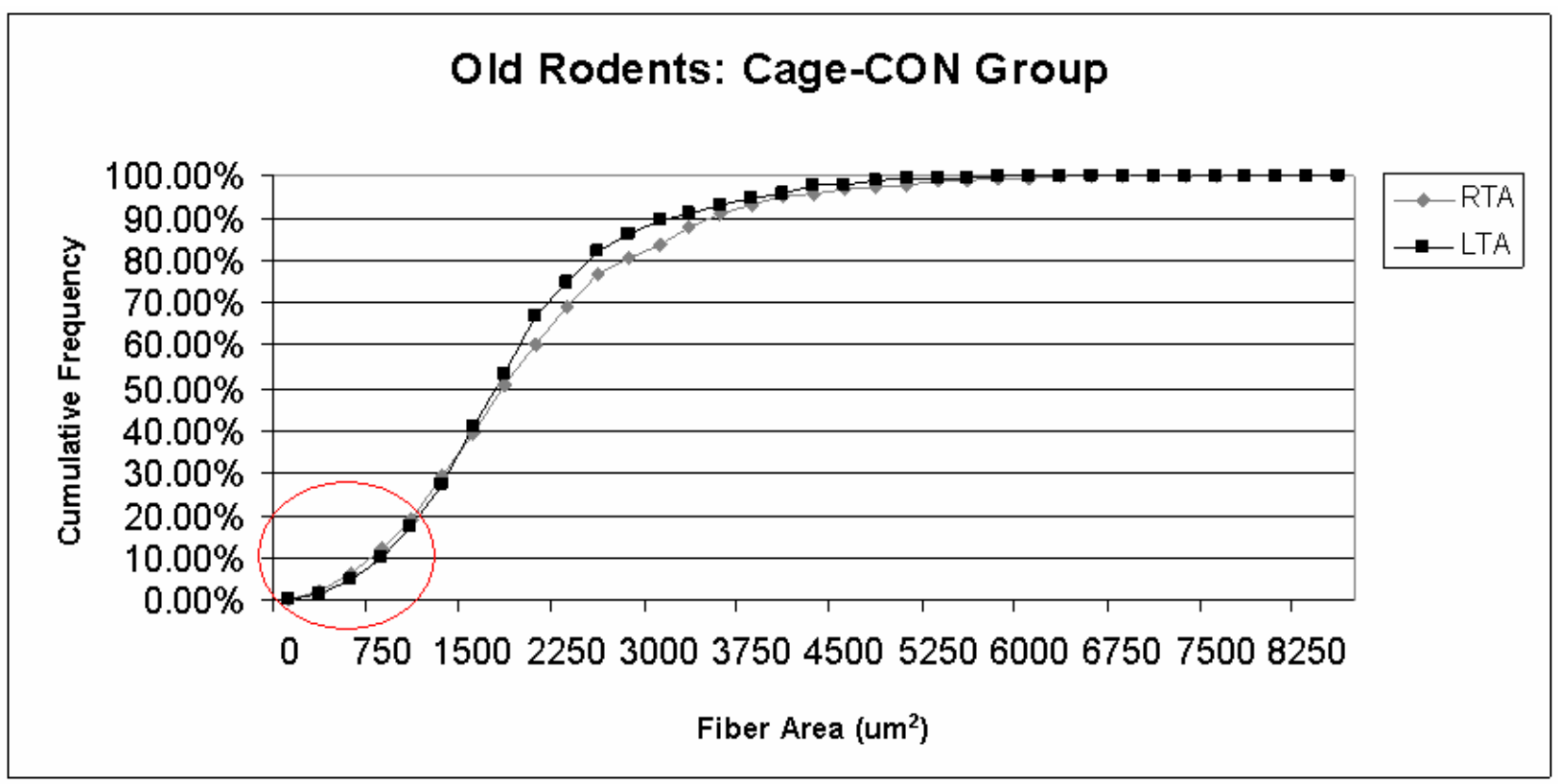


Figure 2a

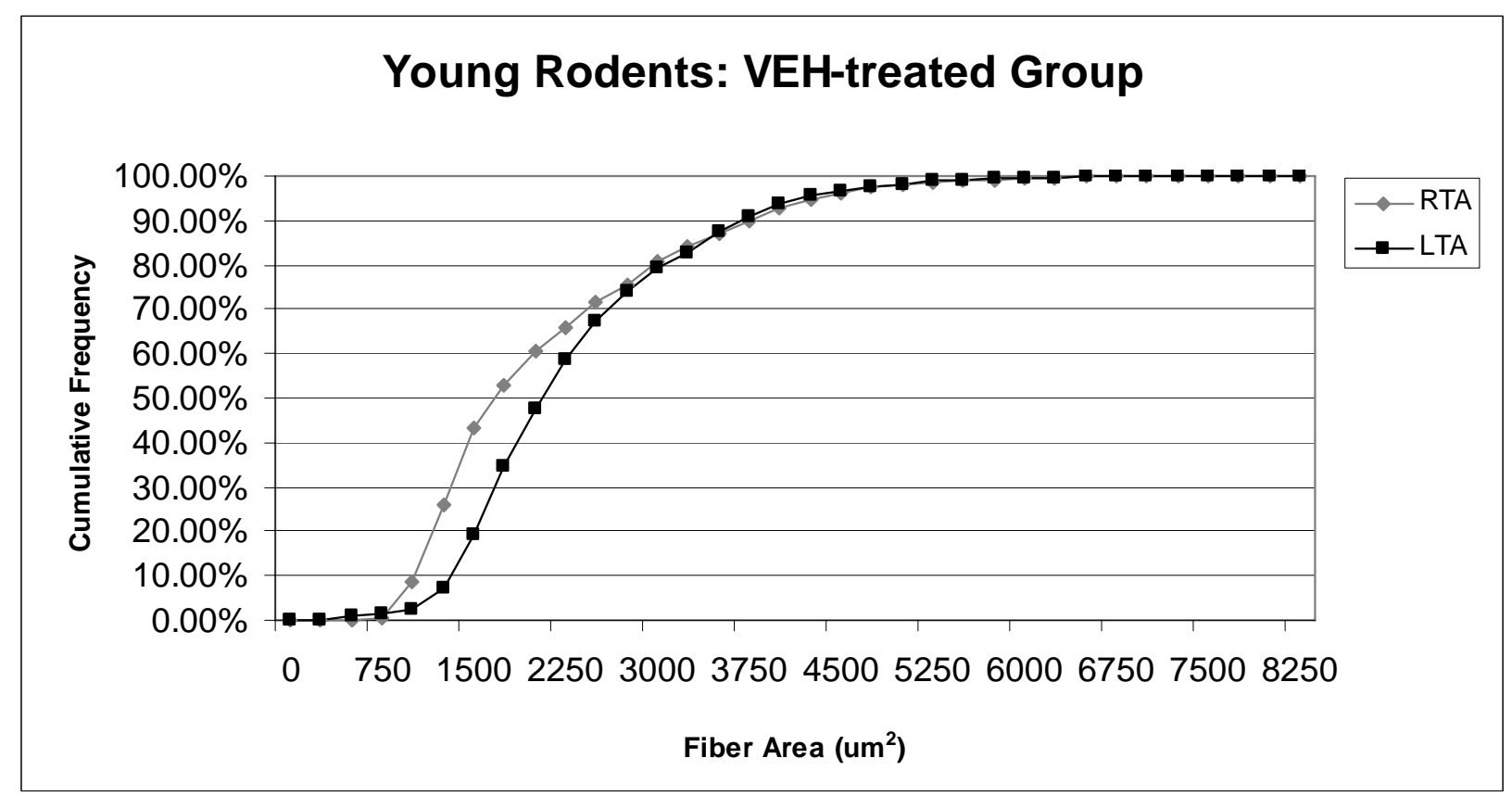

Figure 2b

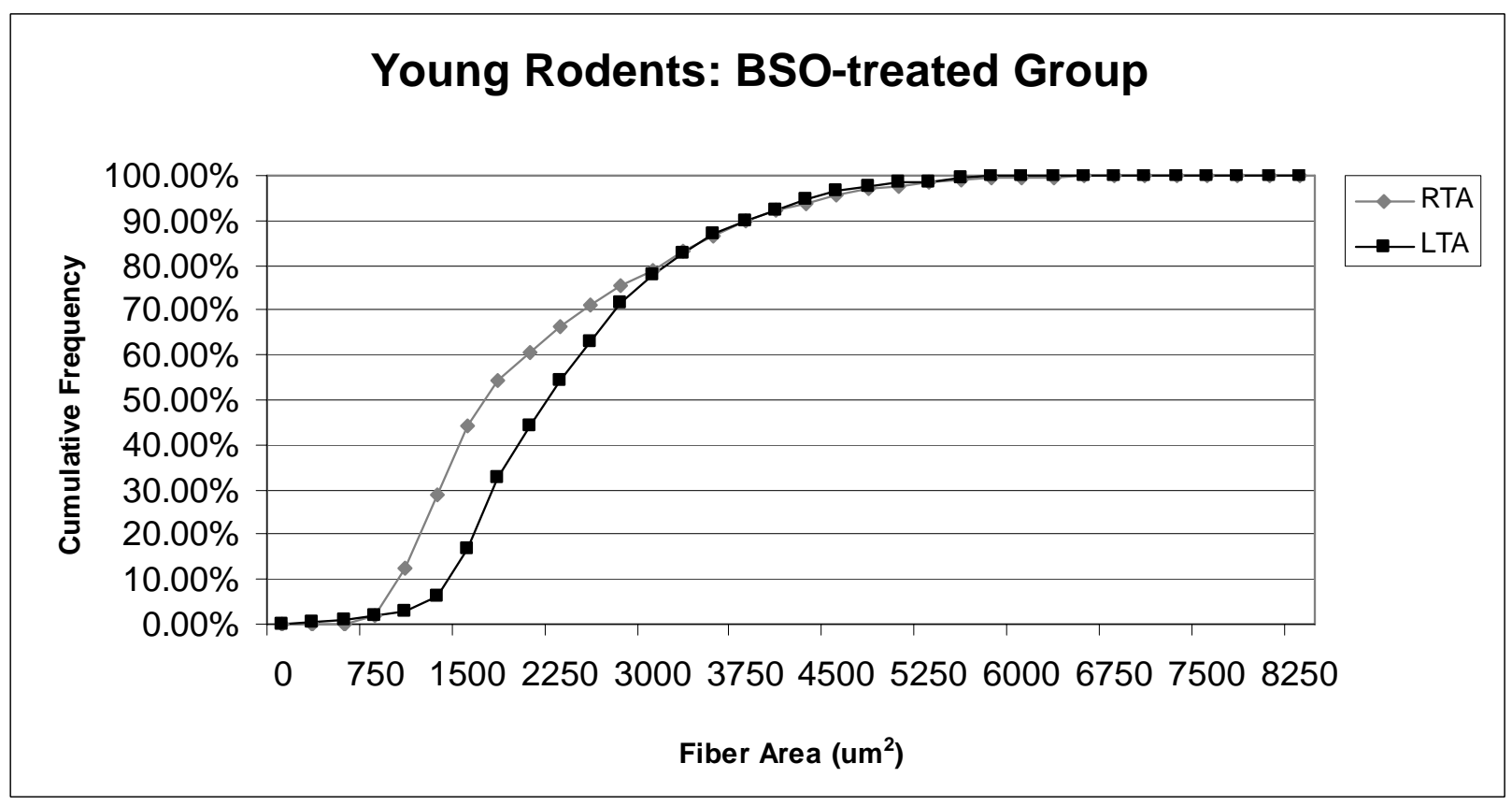


Figure 2c

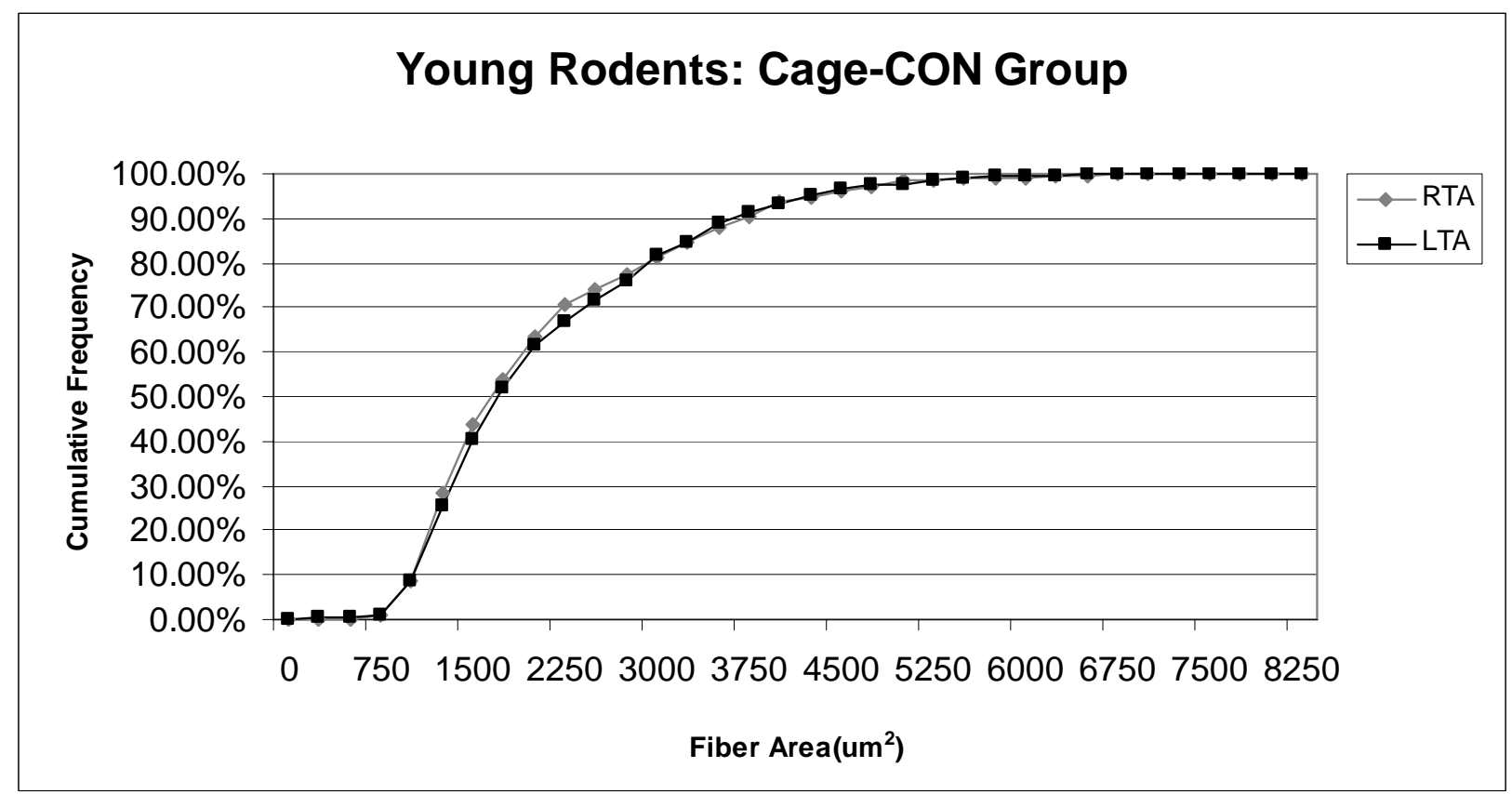


Figure 3 (A-D)

A) Young VEH-treated

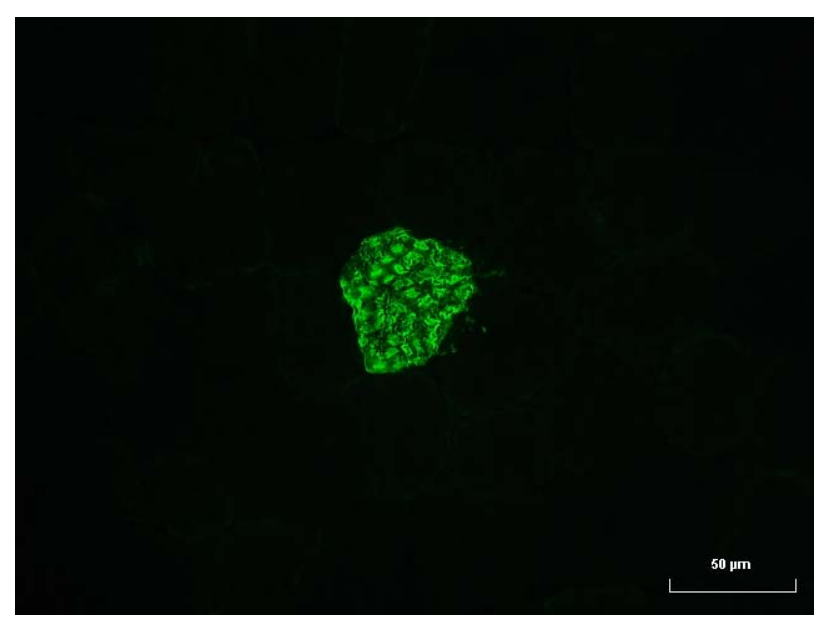

B) Old VEH-treated

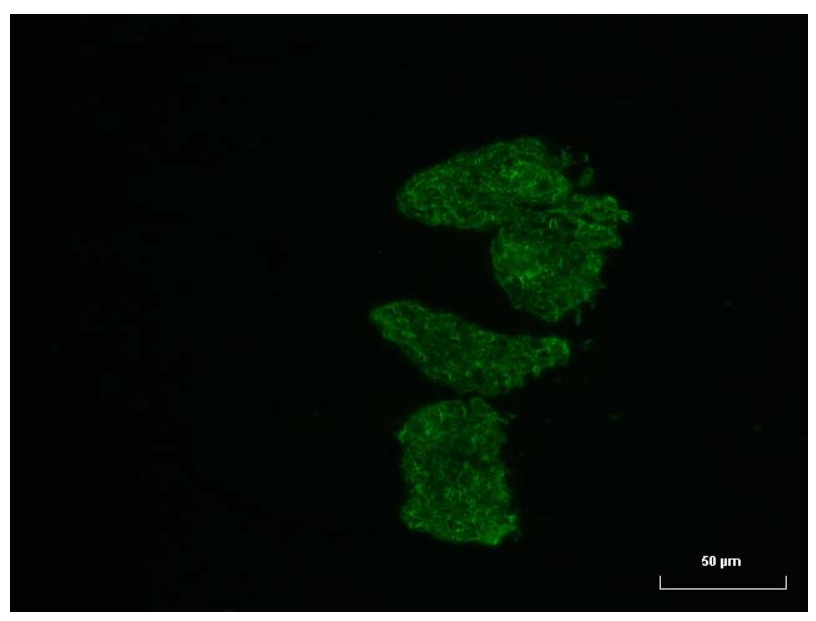

C) Young BSO-treated

D) OLD BSO-treated
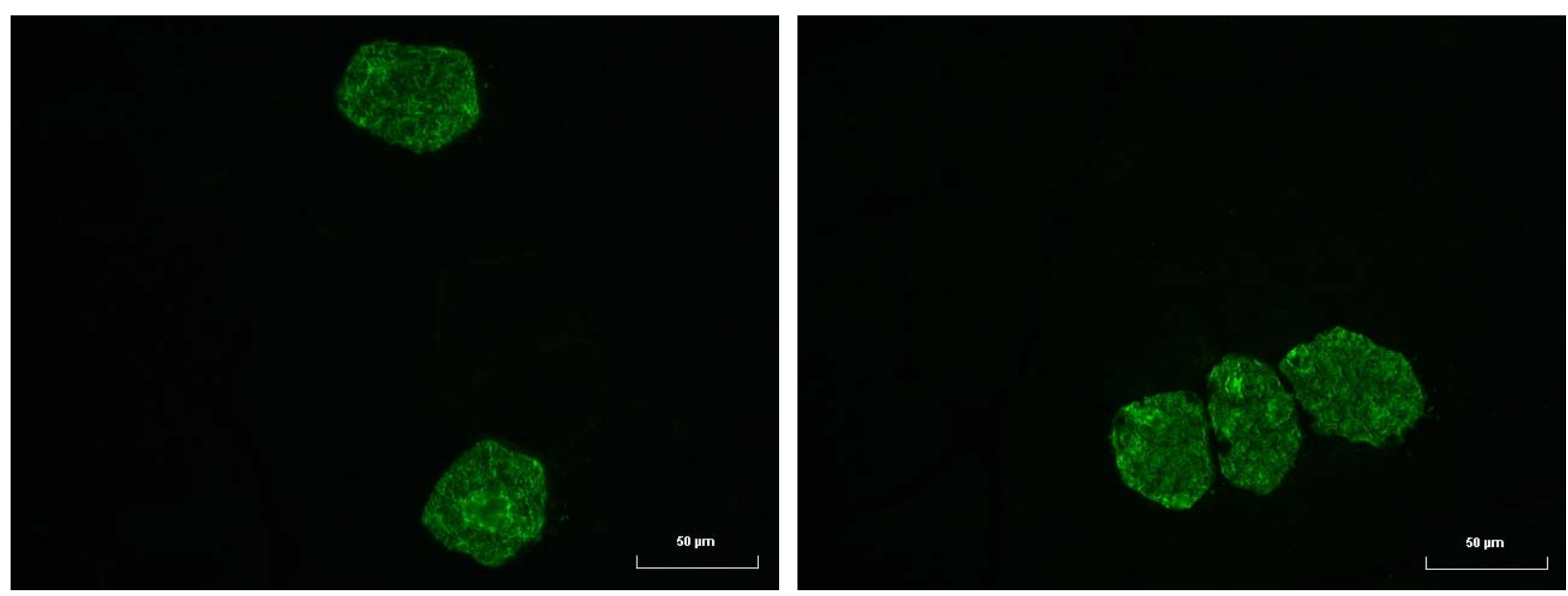
Figure 3 (E-F)

E) Young CON

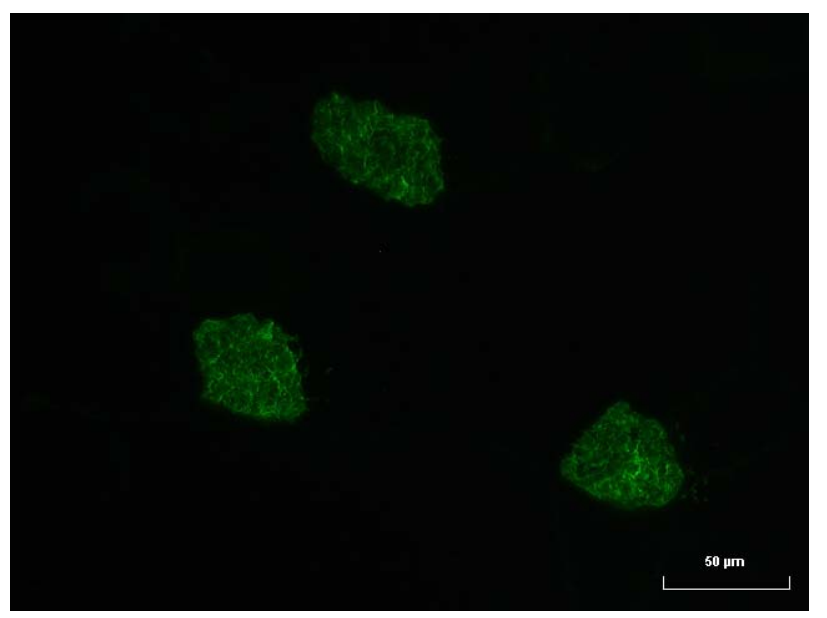

F) Old CON

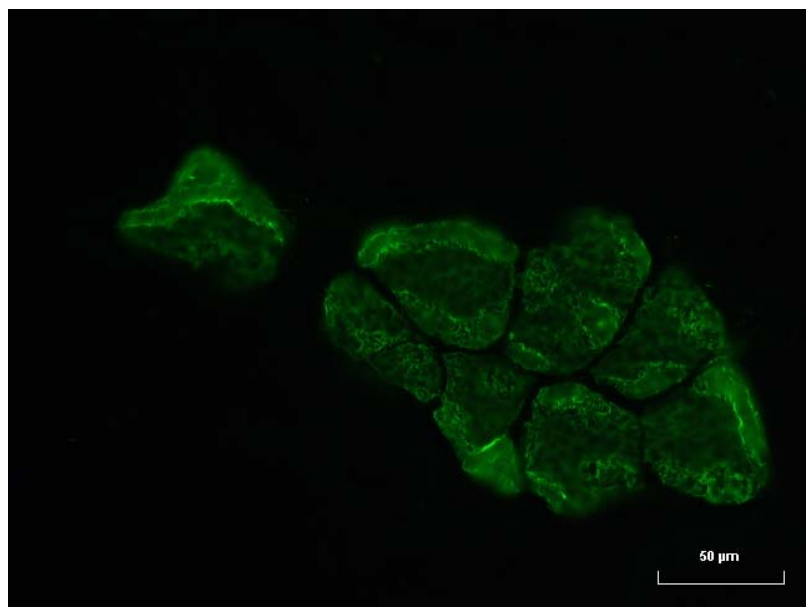


Figure 4

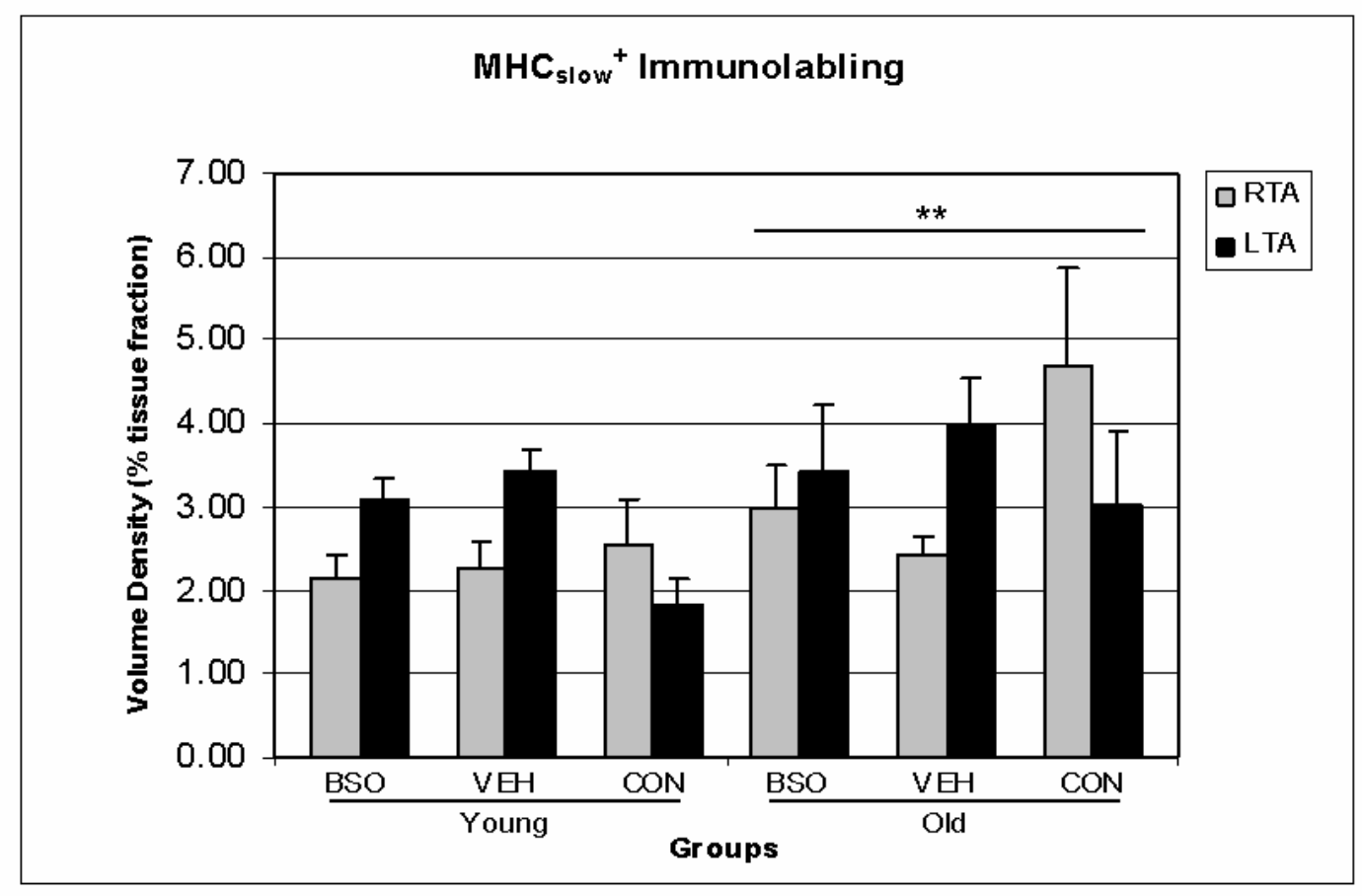


Figure 5 (A-D)

A) Young VEH-treated

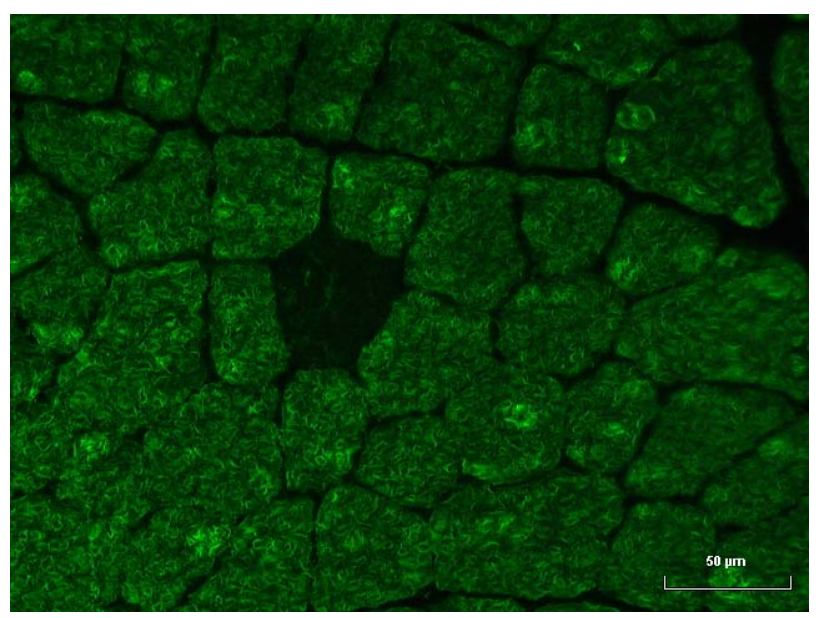

B) Old VEH-treated

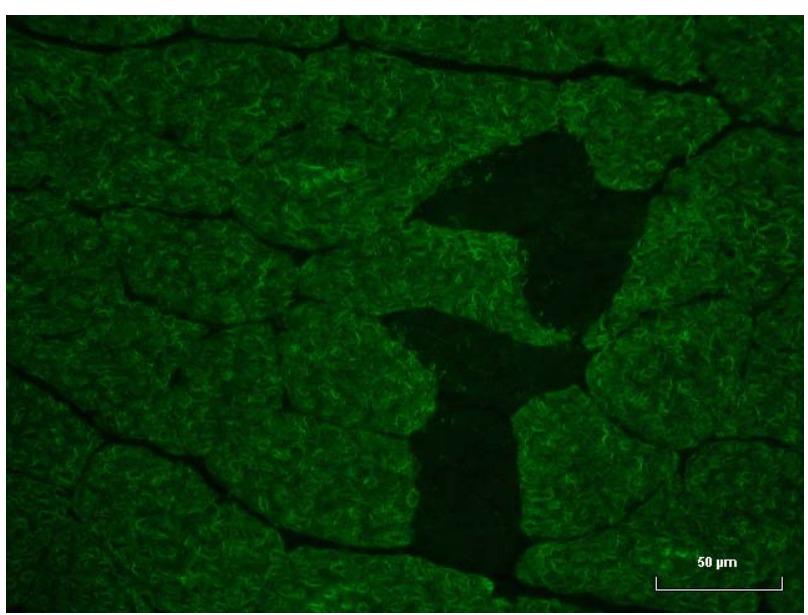

C) Young BSO-treated

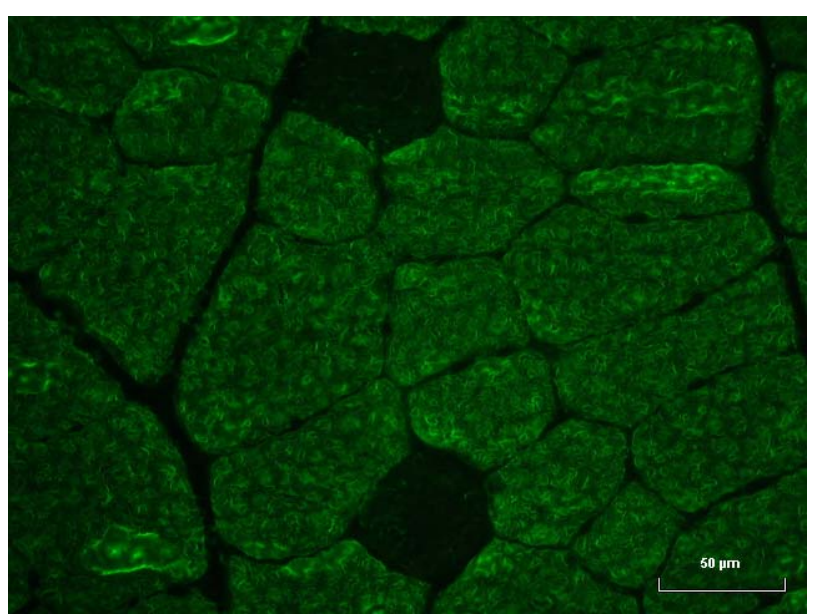

D) Old BSO-treated

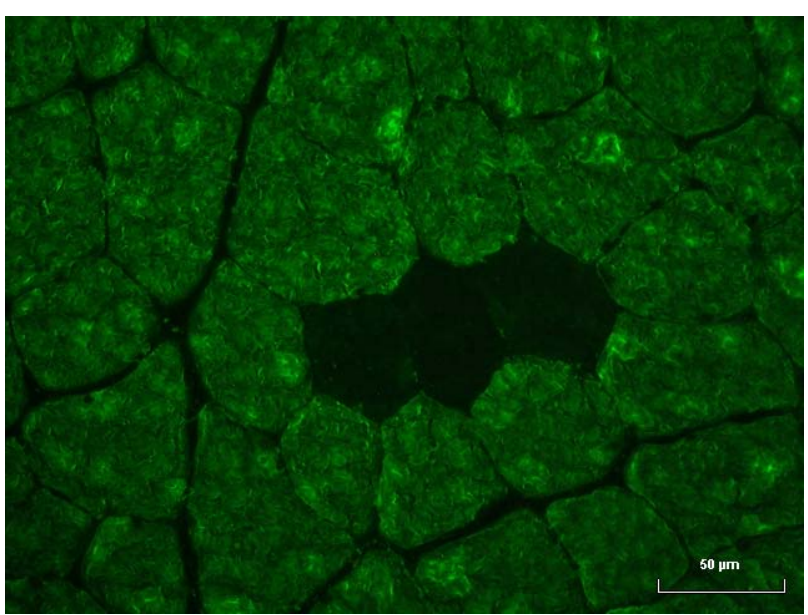


Figure 5 (E-F)

E) Young CON

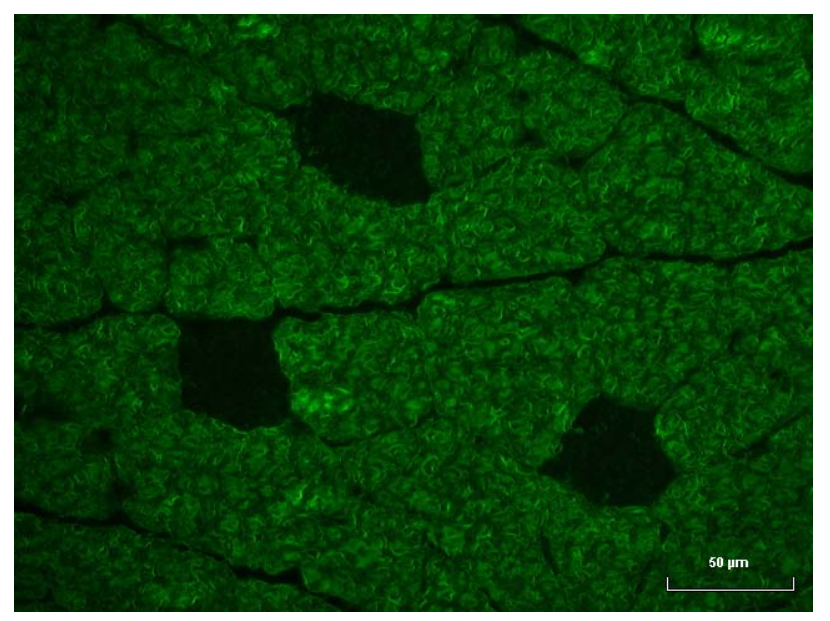

F) Old CON

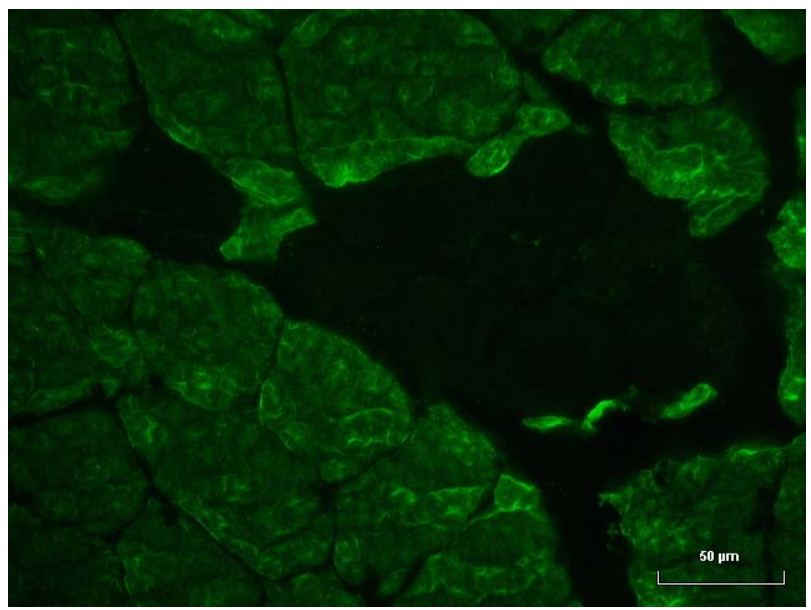


Figure 6

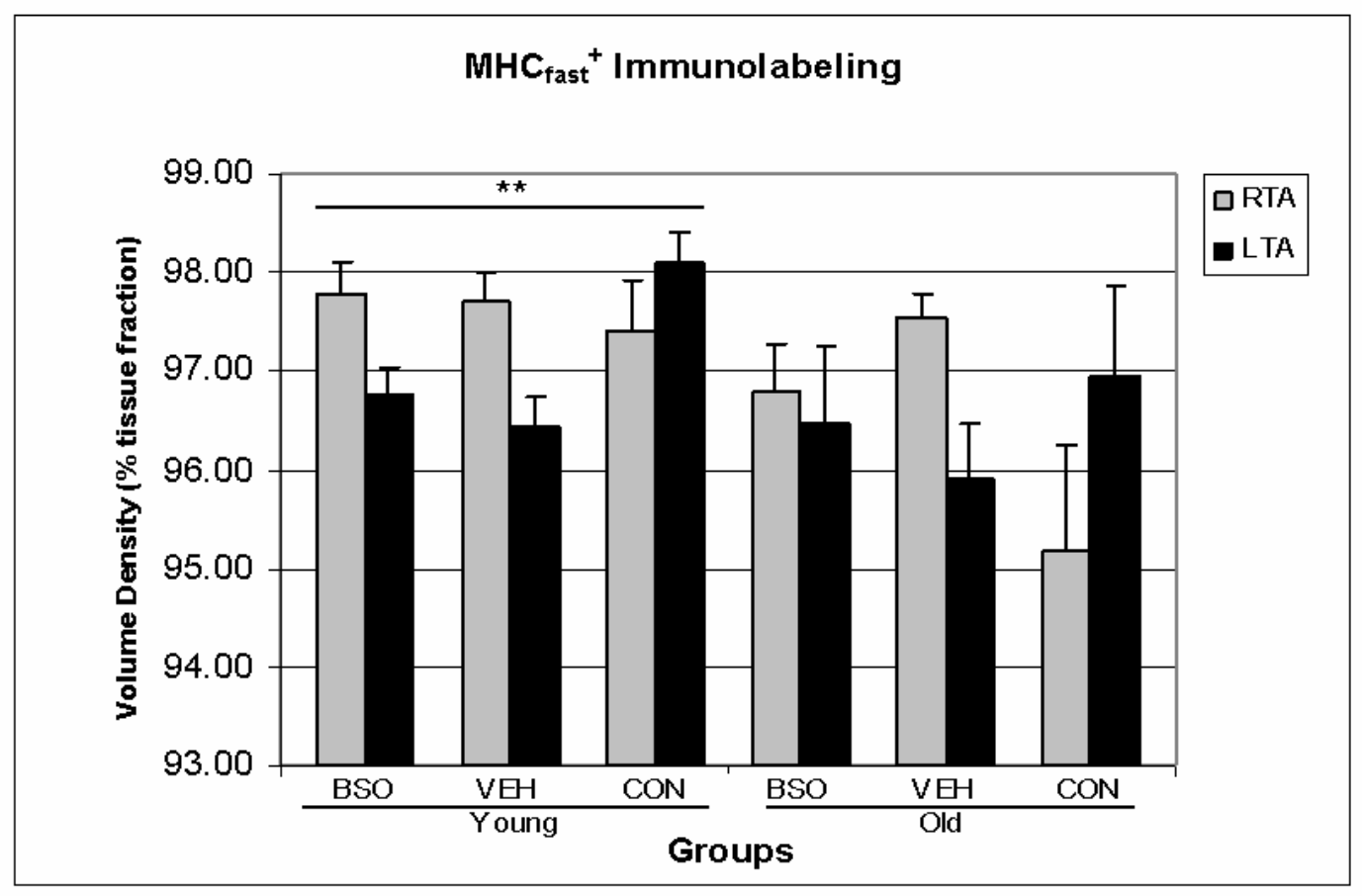




\section{General Discussion:}

Determining and characterizing both time- and dose-dependent responses for skeletal muscle injury and adaptation that follows exposure to both acute and chronic mechanical loading is essential in addressing the current voids and misconceptions. Previous studies have associated the majority of contraction-induced muscle damage into a single phenomenological event. This is not the case, since adaptive responses may occur over a broad continuum even with highintensity mechanical loading exposure/s. The effects of age only make this task more difficult in discriminating and differentiating the time frame, capacity, and mechanisms that are associated with repair, regeneration, and adaptation following mechanical loading exposure/s. In order to determine the influence age has on these effects, we needed to establish measures that adequately represented the events generated by acute and chronic, repetitive SSC mechanical loading in both time and intensity dependent manners, as well as for those exposures that were outside the "normal" injurious state. To do this we characterized and quantified:

1) The effects of acute SSC contraction-induced skeletal muscle injury in both time- and dose-dependent responses.

2) How age influences the acute temporal response to adaptive SSC loading.

3) The effects age has on the adaptive response following repetitive SSC loading.

4) If oxidative stress influences the adaptive response following repetitive SSC loading with aging.

5) The impact age has on acute early events involving muscle regeneration following adaptive SSC loading.

Collectively, these aims allowed us to address current disparities and quantify both performance measures as well as soft-tissue mechanobiology underlying these differential paradigms. 


\section{Acute Contraction-induced Skeletal Muscle Injury:}

Overt skeletal muscle injury has been well documented previously (Armstrong et al., 1983, Armstrong, 1990, Geronilla et al., 2003, McCully and Faulkner, 1985), yet the majority of previous studies (Devor and Faulkner, 1999, Van Der Meulen et al., 1997, Lieber and Friden, 1993) are limited by having reported the number, percent, or cross-sectional area of damaged fibers at single time points following exposure. Also, previous histological studies have failed to directly quantify myofiber degeneration and inflammation and its relationship to evident functional deficits following SSC mechanical loading. It was essential to devise a rapid and sensitive method that would be proficient in establishing highly reproducible results and collectively expand our understanding of contraction-induced muscle injury by determining both time- and dose-dependent responses following mechanical loading. In our initial studies, we reported time-dependent changes that occurred in rodent TA muscle following SSC-induced muscle injury, and that the levels of myofiber degeneration, inflammation, and related changes in the interstitial space using our standardized stereological technique. Additionally, degenerative myofibers and interstitial space changes were associated with functional performance temporally (Baker et al., 2006a). These results are in agreement with data reported previously (McCully and Faulkner, 1985), yet previous histological studies have failed to directly quantify myofiber degeneration and its relationship to evident functional deficits following contraction-induced exposure. While the ability to characterize early-phase muscle injury is essential in understanding skeletal muscle degeneration/regeneration kinetics, it is also important to understand and quantify dose-response characteristics following mechanical loading exposure. For this reason we investigated the effect increasing numbers of SSCs had on muscle performance and morphology. Our results indicate that increasing indices of myofiber 
degeneration and inflammation paralleled the decrease in functional deficit exhibited by the decline in isometric force production in groups exposed to increasing numbers of SSCs (Isometric, $30 \mathrm{SSCs}, 70 \mathrm{SSCs}$, and $150 \mathrm{SSCs}$, respectively). The results indicate an apparent division with respect to the number of SSC repetitions required to induce the subsequent inflammatory cascade and degenerative response, thus surpassing the TA's safety threshold at 70 SSC repetitions. No myofiber degeneration or inflammatory response was observed in the contra-lateral control limb, the isometric-control group, or $30 \mathrm{SSC}$ group. These measures illustrate a clear delineation for the target muscle's safety threshold (or tolerance) with increasing number of repetitions, and that there is a level of exposure where the capacity to withstand the initial injury is compromised, and hence exceeds the muscle's safety threshold. Collectively, time- and dose-dependent factors impact the safety threshold of skeletal muscle and are critical when designing preventative strategies in vocational and recreation arenas and in rehabilitative medicine, as well as for understanding the etiology of acute-loading injuries.

\section{Influence of Age on Skeletal Muscle:}

Throughout the course of one's life, there is an inevitable decline in skeletal muscle mass, and this phenomenon has been termed sarcopenia and has been documented extensively (Evans, 1995, Roubenoff, 2001). Coincidental with the loss of skeletal muscle mass is the decline in function, however whether this is causal or one of the resulting factors is less clear. More specifically, muscle strength declines approximately 15-30 percent in healthy population following the seventh decade of life. Muscle mass decreases approximately 40 percent starting at the third decade with more rapid loss in the later decades of life (Evans, 1995). There are multiple factors associated with the aging process that may be indicative of sarcopenic-like 
changes, which have been previously cited, they are: contraction-induced skeletal muscle injury (Faulkner et al., 1995), increases in the inflammatory milieu as well as specific components of inflammation (Bruunsgaard et al., 1997, Roubenoff et al., 2003, Bruunsgaard et al., 2001), denervation with subsequent reinnervation of motor units principally affecting type II muscle fibers (Pettigrew and Gardner, 1987, Larsson et al., 1979), circulating and muscle-specific modifications in the "growth" hormone profile that drives both muscle growth as well as maintenance (Bross et al., 1999, Tatar et al., 2003), changes in muscle energy metabolism (Evans, 1995), and increased reactive oxygen/nitrogen species (Bejma and Ji, 1999, Weindruch, 1995). More recently mitochondrial dysfunction (Dirks and Leeuwenburgh, 2002, Aiken et al., 2002) and the loss of specific muscle nuclei and myofibers via apoptotic events (Krajnak et al., 2006, Siu and Alway, 2005, Dirks and Leeuwenburgh, 2002) have been implicated as mechanisms contributing to sarcopenia. Irrespective of total contribution, this multitude of variables ultimately is driving healthcare costs related with sarcopenia to new heights, especially since the population in the United States is growing considerably older. The workforce in the United States is aging too, for approximately 20 percent of the labor force is comprised of workers over the age of 55 ((NRC) and (IM), 2001). Thus, sarcopenic changes with aging are detrimental, not only with activities associated with daily living but also with more arduous labor-intensive tasks.

Numerous countermeasures exist that have been suggested in mitigating the deleterious effects of the aging process while concurrently improving the quality of life, and these actions must be put into practice to enhance muscle growth/maintenance and functional output. Indeed, daily physical activity (aerobic and/or anaerobic) coupled with modifications in dietary intake may ultimately provide the greatest protection against the rapidity of these inevitable losses. 
Additionally, understanding the focal mechanisms contributing to the commencement of sarcopenia will particularly impact how clinicians implement individual therapeutic strategies.

\section{Adaptive Mechanical Loading Paradigm and the Influence of Age:}

The best known strategy to increase skeletal muscle mass is with mechanical exposures (resistance/weight training), however, prescribing this mode of training is cautioned in aged populations due to the general condition of the older individual - sarcopenic state. Yet, our results indicate that not all high-intensity mechanical loading produces overt muscle damage, for this is exemplified by skeletal muscle performance and morphology in both young and old rodents following both acute and chronic mechanical loading that display different characteristics than that typically observed following "normal" contraction-induced muscle injury. Here we sought to mimic the results of high-intensity resistance training that have been reported in exercise/athletic populations, which have investigated the ability of young and old subjects to increase muscle hypertrophy (Welle et al., 1996, Ivey et al., 2000). In order to advance these previous findings, it was our intent to illustrate possible factors involved in the adaptive phase following chronic exposure and the influence of oxidant/antioxidant profile and age. Since the loss of skeletal muscle will directly impact skeletal muscle force production (muscle strength and power), administration of chronic mechanical loading exposures should be utilized to create an adaptive environment in which muscle fiber hypertrophy would be advantageous. One caveat that exists with respect to the historical perspective involving highintensity mechanical loading is the propensity to induce overt skeletal muscle damage. Recently, we have reported that this overt muscle damage does not occur, regardless of age in male Fischer 344 x Brown Norway F1 rats that were exposed to adaptive SSCs chronically (Cutlip et al., 
2006). Importantly, rodents that were administered an acute exposure of the same protocol paradigm also exhibited minimal myofiber degeneration $(<1 \%$ tissue fraction). Moreover, this is in contrast with rodents exposed to injurious SSCs from our lab previously, which have exhibited pronounced muscle degeneration following exposure ( $\sim 10 \%$ tissue fraction). Once again, in this dissertation we are reporting that overt muscle damage (myofiber degeneration/necrosis) not only does not occur with any significance, but should not be regarded as a limiting factor when designing and prescribing resistance exercise regimens for healthy-aged populations (those aged populations that present with minimal risk factors or comorbidities). Moreover, optimizing the biomechanical loading signature and the intensity of the mechanical loading exposure for an individual is paramount especially in those individuals presenting with risk factors associated with disease states, so that they too may experience the benefits of resistance exercise. Supporting this assertion is that in the current model, electrodes activate the target muscle via supra-maximal electrical stimulation, an intensity that will seldom (if ever) be attained in human populations. The absence of an obvious degenerative effect observed following high-intensity mechanical loading in this and recent studies (Cutlip et al., 2006) (Baker et al., In preparation 2007) is very fascinating, since most published literature has grouped the "muscle injury response" following this mode of exposure with that response of an overt muscle injury. Thus, the approach used in this dissertation should be considered using a translational approach with respect to human populations, since very recent reports in older men and women exposed chronically to high-intensity resistance training concluded that this mode of exposure may be the most advantageous for improving the quality of life in aged populations (Hartman et al., 2007). Yet, a caveat does exist in that the dynamometer drives the activated muscle action, compared to volitional movements in humans and rodents which are under control of the central 
nervous system. Furthermore, even though the physiological "driver" is replaced in the scenario of in vivo dynamometry (non-volitional exposure), the resulting advantage is quite apparent since the biomechanical loading parameters used to induce the exposure are under complete control, irrespective of voluntary initiative (voluntary exposure). This results in precise control over the exposure without confounding factors such as central drive, muscle activation, and fiber recruitment patterns influencing the resulting exposure and responses. Thus, from a basic biological/physiological "worst case scenario" (supra-maximal electrical stimulation and a deprived host state) in our current work, not all mechanical loading of skeletal muscle will result in overt damage. Furthermore, this may lead to improved maintenance of functional measures via plastic changes occurring within the target muscle, yet the clear void of human studies is that they often do not investigate biological changes within the target muscle.

\section{Influence of Oxidative Stress:}

Even though the current investigation does not support the contention that glutathione depletion negatively affects the adaptive process in young or old rodents, various pathological conditions have implicated and shown causal relationships for the involvement of oxidative stress in disease manifestation and progression, including aging (Warner, 1994, Toscano et al., 2005, Sohal and Weindruch, 1996, Rodriguez and Tarnopolsky, 2003, Houstis et al., 2006, Bohr and Anson, 1995). Irrespective of disease state, it is apparent that tissue damage from oxidative stress ensues following an imbalance or disturbed state between pro and antioxidant activity. With regards to skeletal muscle, sources of oxidative stress are numerous and are the consequences of both nonenzymatic and enzymatic chemical reactions. As well, both oxygen and nitrogen containing radicals and oxygen and nitrogen containing non-radicals participate in oxidative stress-induced 
tissue damage. It is important, however, to understand that oxidative stress also serves as a stimulus for and a regulator of signal transduction pathways, membrane integrity, enzymatic control, protein function, reduction-oxidation reactions (redox balance), and tissue adaptation following mechanical exposure/s (Urso and Clarkson, 2003). Since these oxidative products may be both extra- and intra-cellularly derived, pinpointing the exact location as well as contribution of any particular agent is difficult. Besides determining the effects of glutathione depletion has on oxidative stress production and it's involvement in chronic mechanical loading exposures, there are other contributing sources of oxidative stress that may have be involved following the SSC loading of skeletal muscle: 1) nicotinamide adenine dinucleotide phosphate (NADPH) oxidase, 2) xanthine oxidase, 3) nitric oxide, and 4) mitochondrial generated oxidative stress.

1) NADPH is an enzyme complex that resides at the plasma membrane in various cell types (myocytes, neutrophils, monocytes, and macrophages) that produces increased amounts of superoxide by catalyzing the transfer of a single electron from the reduced form of NADPH or NADH to oxygen. It has been reported that the NADPH oxidase that resides in skeletal muscle is variably located. Such sites include the sarcolemna (Javesghani et al., 2002) and the sarcoplasmic reticulum (Xia et al., 2003), where the former has been suggested to be involved in signal transduction and the latter has been suggested to be involved in $\mathrm{Ca}^{2+}$ regulatory events and muscle contraction (Xia et al., 2003). Additionally, NADPH oxidase is found in the mitochondria of cells as well as in neutrophils, monocytes, and macrophages where increased amounts of superoxide are produced and may have deleterious effects (Fridovich, 1995). 
2) Xanthine oxidase (as well as xanthine dehydrogenase are interconvertible forms of the same gene product - xanthine oxidoreductase) catalyzes the formation of hydrogen peroxide and superoxide from purine substrates such as xanthine and hypoxanthine (Borges et al., 2002). Primarily located in the cytosol, xanthine oxidoreductase can be found in various tissues including liver, heart, and skeletal muscle and this is where the potential to cause oxidative stress-induced damage occurs (Saugstad, 1996).

3) Nitric oxide synthase (NOS) is present in at least three distinct isoforms: 1) neuronal NOS, 2) inducible NOS, and 3) endothelial NOS (Stamler and Meissner, 2001). nNOS is the predominant form of nitric oxide in skeletal muscle, and exists in the alternatively spliced form nNOS $\mu$ (Silvagno et al., 1996), and is anatomically located at the subsarcolemma and is associated with the dystrophin complex. It has been suggested that nNOS in skeletal muscle may influence excitation-contraction coupling, regulation of blood flow, mitochondrial energy production, and glucose metabolism (Stamler and Meissner, 2001).

4) Another very important source of oxidative stress production is the mitochondrial electron transport chain, specifically at complexes I (via NADH / CoQ oxidoreductase) and III (via Ubiquinol / Cytochrome c oxidoreductase) (Jezek and Hlavata, 2005). Damage to the mitochondria resulting from oxidative stress and that which occurs during aging may be a result of mitochondria complex II damage (via succinate dehydrogenase) (Zhang et al., 1998). Collectively, these complexes result in the increased production of superoxide radicals, which is then converted to hydrogen peroxide. Hydrogen peroxide then may be converted to the more damaging hydroxyl 
radical. As a result, hydrogen peroxide and the hydroxyl radical may target more specific anatomical sites that lead to preferential damage of proteins, lipids, and/or DNA. Although many studies have investigated the impact oxidative stress (as well as the counterregulatory effects of antioxidants) has with respect to aerobic exercise exposure/s, few studies have investigated the relationship between oxidative stress and mechanical loading (resistance exercise). Even though acute mechanical loading exposures generally result in increased myofiber degeneration and inflammation (Baker et al., 2006b, Tamaki et al., 1997) it is unknown if oxidative stress is a prerequisite with mechanical loading of skeletal muscle, and if it is associated with inflammation and myofiber degeneration that ultimately ensue. Very recently a study by Uchiyama and colleagues addressed this question, and showed that ROS-induced muscle damage was a consequence of weight-lifting exercise (Uchiyama et al., 2006). In this case, not only was the ischemia-reperfusion response similar to that observed in humans, but Uchiyama and colleagues also showed that mechanical loading of skeletal muscle does in fact lead to an anoxic state with ROS production, as confirmed indirectly by significant increases in antioxidant activities of the enzymes superoxide dismutase, glutathione peroxidase, and catalase. Further, the investigators concluded that muscle cell membrane microdamage may play an important role in weight-lifting-induced muscle damage in addition to the initial mechanical damage. This was hypothesized to be due to both immediate and secondary changes that were observed in antioxidant profiles. Initially, increased antioxidant enzyme activity would have been expected due to ischemic-reperfusion state that elicited ROS production in the active muscle. Secondarily, changes in antioxidant enzyme activity days following (24-72 hours) the exercise exposure would have relied upon migration of cellular infiltrates (neutrophils and macrophages) into the injured muscle to aid in repair and regenerative states. Since oxidative 
stress' involvement in muscle damage is plausible, we wanted to consider the impact that oxidative stress may have on chronic loading with aging. To do this we modulated the antioxidant profile, which we believed would be most successfully achieved by manipulating the glutathione system. Glutathione is conceivably the most influential antioxidant/antioxidant system in vivo, and skeletal muscle represents the largest pool of total glutathione in the body. Given this fact, glutathione greatly impacts multiple pathways and functions acting directly as a reducing agent, serving as a substrate for glutathione peroxidase (and other enzymes), and functioning in the recycling of radicals generated from other molecules; thus the adaptive response ensuing from manipulating the glutathione system may be ascertained with respect to age and chronic mechanical exposures. Even though depletion of the glutathione system has been shown to negatively impact exercise performance in young mice (Leeuwenburgh and Ji, 1995), we found little impact with treatment, irrespective of age. Furthermore, exercise training has not been beneficial in countering oxidative stress in aged, senescent muscle mostly because aging negatively affects increases in antioxidant potential that are typically observed following exercise training (Leeuwenburgh et al., 1994). Depleting the glutathione status of the TA muscle did not impair muscle hypertrophy, irrespective of age. Our results demonstrate that in aged rodents there is a deleterious effect that severely limits the muscle's hypertrophic response to mechanical loading. However, we suggest this detrimental effect does not depend on glutathione status.

\section{Influence of Age on Skeletal Muscle Regenerative Capacity Following Mechanical Loading:}

Based on the cumulative morphological changes following the 4.5 week SSC protocol, it is evident a limited capability exists for SSC-induced adaptation to occur in aging skeletal muscle. 
Indeed, morphological adaptation is considerably limited and we suggest that this is due to the initial impediment of the regenerative process following a single mechanical exposure. Our results indicate that the expression of developmental MHC in individual TA muscle fibers is reduced in old rodents compared with young counterparts following a single bout of SSCs. In addition, the use of supramaximal electrical stimulation does not appear to influence the developmental MHC expression profile in myofibers, since volitional studies have reported comparable incidences of myofibers labeling positively for developmental MHC following mechanical exposure in young rodents (Smith et al., 1999). Even though aged muscle does appear to have the capacity for adaptation (as old rodents did display increased developmental MHC labeling in the exposed TA muscle compared with the non-exposed limb), this response is clearly diminished compared to exposed TA muscles from young rodents. Furthermore, this diminished signal in myofibers of old rodents that are stimulated to undergo adaptation, may suggest why old rodents do not have the capacity to adapt to chronic exposures when compared with young counterparts. When a chronic, repetitive bout of SSCs is administered over a 4.5 week period there is minimal developmental MHC expression in young and old rodents, yet young rodent's exhibit marked adaptation following the exposure. When oxidative stress' influence on muscle adaptation was examined following chronic SSC exposure, it was shown that the adaptive response is not influenced by depleting the glutathione system. We cannot rule out the role of other oxidant/antioxidant systems that may be initiated or engaged during the specific manipulation of a given component, thus, these compensatory factors need to be explored further.

In summary, we have illustrated that there is a differentiation which clearly exists 
between overt skeletal muscle injury (classically defined as eccentric- or contraction-induced muscle injury) and adaptive muscle contractions (acute and chronic SSCs that incorporate eccentric movements). In this report, we 1) quantified (using a novel stereological approach in skeletal muscle) and characterized both time- and dose-dependent effects of skeletal muscle following acute, injurious SSCs in young rodents, and 2) illustrated the influence of age on the adaptive response following acute mechanical loading. Further, we 3) examined and quantified the influence age has on chronic SSC loading, and 4) if oxidative stress influences the chronic adaptive response in the absence of overt skeletal muscle injury. Finally, we 5) investigated the acute events that are involved in skeletal muscle regeneration following adaptive SSC loading, and considered the role aging has on this process. Our results from this final objective indicate initial muscle regeneration is a critical element in assuring successful adaptation, thus investigating the mechanisms involved in initiating successful muscle regeneration following exposure will be beneficial in aging populations. Again, it is essential and cannot be understated that not all acute and chronic mechanical loading (specifically loading comprised of eccentric movements) results in overt skeletal muscle damage, which has been reported for more than twenty years. Remarkably the capacity to respond efficiently to an initial mechanical stimulus as one ages, may be one of the most important factors that ultimately regulates adaptation of skeletal muscle. Optimizing the initial exposure for maximum adaptation (i.e. number of repetitions, intensity, duty cycle, velocity, etc.) as well as improving the muscle's host environment with age (i.e. via supplements, therapeutic agents, etc.) may improve the responsiveness of skeletal muscle to acute and chronic exposure that has currently been shown to be negatively influenced by age. Finally, as chronic exposure of skeletal muscles to highintensity mechanical loading has been shown to be the most desirable means to attenuate the 
effects of sarcopenia (Lambert and Evans, 2005, Evans, 1995), it too may be the most effective and appealing mode of physical activity to counteract the effects often observed with musculoskeletal dysfunction in the workplace.

\section{$\underline{\text { Future Research }}$}

The direction of future research stemming directly from this current work becomes an enviable task to any researcher. Deciphering the exact mechanism/s that contributes to the diminished age-related adaptation following mechanical loading is paramount. Furthermore, it will be fascinating to discern whether there is a distinct mechanism (i.e. a decreased recapitulation of the developing muscle's signal) that limits adaptability, or more probable, the interaction of various mechanisms that lead to the overall reduction in muscle adaptation following acute and chronic mechanical exposure/s that is incurred with age. The captivating and very elusive question is can we do something or anything about this limitation? A variety of countermeasure exist in warding off the negative effects of age-induced muscle loss, however it is plausible that with a clearer understanding of the mechanistic control of this loss we may employ mechanism-specific approaches that will better offset this detrimental effect. Since mechanical loading is very specific in nature, involving only the local muscle/s that undergoes exposure, systemic factors will exert a lesser role in the overall adaptive response. Thus, one of the most appropriate steps in realizing the contribution of exact factors involved in this process is to investigate and gain a more comprehensive awareness of how local factors mediate cell signaling and gene expression, growth and regeneration, and cell death and survival. Variables that have influencing effects on the factors mentioned above are: physical activity (various modes applied independently or in combination), pharmacological agents (either alone or in combination), therapeutic modalities 
used in clinical settings (ice, heat, electrical stimulation, ultrasound, and massage to name only a few) and the myriad of nutrition and supplement interventions. Investigating the beneficial (as well as possible detrimental) effects of these factors, and their efficacy in improving muscle adaptation with aging should be a principal focus in future research. 


\section{$\underline{\text { References }}$}

(NRC), N. R. C. \& (IM), I. F. M. (2001) Musculoskeletal Disorders and the Workplace, Washington, D.C., National Academy Press.

AIKEN, J., BUA, E., CAO, Z., LOPEZ, M., WANAGAT, J., MCKENZIE, D. \& MCKIERNAN, S. (2002) Mitochondrial DNA deletion mutations and sarcopenia. . Ann NY Acad Sci, $959,412-423$.

ARMSTRONG, R. B. (1990) Initial events in exercise-induced muscular injury. Med Sci Sports Exerc, 22, 429-35.

ARMSTRONG, R. B., OGILVIE, R. W. \& SCHWANE, J. A. (1983) Eccentric exercise-induced injury to rat skeletal muscle. J Appl Physiol, 54, 80-93.

BAKER, B. A., MERCER, R. R., GERONILLA, K. B., KASHON, M. L., MILLER, G. R. \& CUTLIP, R. G. (2006a) Stereological analysis of muscle morphology following exposure to repetitive stretch-shortening cycles in a rat model. Appl Physiol Nutr Metab, 31, 16779.

BAKER, B. A., RAO, K. M., MERCER, R. R., GERONILLA, K. B., KASHON, M. L., MILLER, G. R. \& CUTLIP, R. G. (2006b) Quantitative histology and MGF gene expression in rats following SSC exercise in vivo. Med Sci Sports Exerc, 38, 463-71.

BEJMA, J. \& JI, L. L. (1999) Aging and acute exercise enhance free radical generation in rat skeletal muscle. J Appl Physiol, 87, 465-70.

BOHR, V. \& ANSON, R. (1995) DNA damage, mutation and fine structure DNA repair in aging. Mutat Res, 338, 25-34.

BORGES, F., FERNANDES, E. \& ROLEIRA, F. (2002) Progress towards the discovery of xanthine oxidase inhibitors. . Curr Med Chem, 9, 195-217.

BROSS, R., JAVANBAKHT, M. \& BHASIN, S. (1999) Anabolic interventions for agingassociated sarcopenia. . J Clin Endocrinol Metab, 84, 3420-3430.

BRUUNSGAARD, H., GALBO, H., HALKJAER-KRISTENSEN, J., JOHANSEN, T. L., MACLEAN, D. A. \& PEDERSEN, B. K. (1997) Exercise-induced increase in serum interleukin-6 in humans is related to muscle damage. Journal of Physiology, 499, 833-41.

BRUUNSGAARD, H., PEDERSEN, M. \& PEDERSEN, B. K. (2001) Aging and proinflammatory cytokines. . Curr Opin Hematol, 8, 131-136. 
DEVOR, S. T. \& FAULKNER, J. A. (1999) Regeneration of new fibers in muscles of old rats reduces contraction-induced injury. J Appl Physiol, 87, 750-6.

DIRKS, A. \& LEEUWENBURGH, C. (2002) Apoptosis in skeletal muscle with aging. Am J Physiol Regul Integr Comp Physiol, 282, 519-527.

EVANS, W. J. (1995) What is sarcopenia? J Gerontol A Biol Sci Med Sci, 50, 5-8.

FAULKNER, J. A., BROOKS, S. V. \& ZERBA, E. (1995) Muscle atrophy and weakness with aging: contraction-induced injury as an underlying mechanism. J Gerontol A Biol Sci Med Sci, 50 Spec No, 124-9.

FRIDOVICH, I. (1995) Superoxide radical and superoxide dismutases. Annu Rev Biochem, 64, 97-112.

GERONILLA, K. B., MILLER, G. R., MOWREY, K. F., WU, J. Z., KASHON, M. L., BRUMBAUGH, K., REYNOLDS, J., HUBBS, A. \& CUTLIP, R. G. (2003) Dynamic force responses of skeletal muscle during stretch-shortening cycles. Eur J Appl Physiol, 90, 144-53.

HARTMAN, M. J., FIELDS, D. A., BYRNE, N. M. \& HUNTER, G. R. (2007) Resistance training improves metabolic economy during functional tasks in older adults. $J$ Strength Cond Res, 21, 91-95.

HOUSTIS, N., ROSENT, E. \& LANDER, E. (2006) Reactive oxygen species have a causal role in multiple forms of insulin resistance. Nature, 440, 944-948.

IVEY, F. M., ROTH, S. M., FERRELL, R. E., TRACY, B. L., LEMMER, J. T., HURLBUT, D. E., MARTEL, G. F., SIEGEL, E. L., FOZARD, J. L., JEFFREY METTER, E., FLEG, J. L. \& HURLEY, B. F. (2000) Effects of age, gender and myostatin genotype on the hypertrophic response to heavy resistance strength training. J Gerontol A Biol Sci Med Sci, 55, 641-648.

JAVESGHANI, D., MAGDER, S. A., BARREIRO, E., QUINN, M. T. \& HUSSAIN, S. (2002) Molecular characterization of a superoxide-generating NAD $(\mathrm{P}) \mathrm{H}$ oxidase in the ventilatory muscles. Am J Respir Crit Care Med, 165, 412-418.

JEZEK, P. \& HLAVATA, L. (2005) Mitochondria in homeostasis of reactive oxygen species in cell, tissues, and organism. Int J Biochem Cell Biol, 37, 2478-2503.

KRAJNAK, K., WAUGH, S., MILLER, R., BAKER, B., GERONILLA, K., ALWAY, S. E. \& CUTLIP, R. G. (2006) Proapoptotic factor bax is increased in satellite cells in the tibialis anterior muscles of old rats. Muscle Nerve, 24, 720-730. 
LAMBERT, C. P. \& EVANS, W. J. (2005) Adaptations to aerobic and resistance exercise in the elderly. Rev Endocr Metab Disord, 6, 137-143.

LARSSON, L., GRIMBY, G. \& KARLSSON, J. (1979) Muscle strength and speed of movement in relation to age and muscle morphology. J Appl Physiol, 46, 451-456.

LEEUWENBURGH, C., FIEBIG, R., CHANDWANEY, R. \& JI, L. L. (1994) Aging and exercise training in skeletal muscle: responses of glutathione and antioxidant enzyme systems. Am J Physiol, 267, R439-45.

LEEUWENBURGH, C. \& JI, L. L. (1995) Glutathione Depletion in Rested and Exercised Mice: Biochemical Consequence and Adaptation. Arch Biochem Biophys, 316, 941-949.

LIEBER, R. L. \& FRIDEN, J. (1993) Muscle damage is not a function of muscle force but active muscle strain. J Appl Physiol, 74, 520-6.

MCCULLY, K. K. \& FAULKNER, J. A. (1985) Injury to skeletal muscle fibers of mice following lengthening contractions. J Appl Physiol, 59, 119-26.

PETTIGREW, F. P. \& GARDNER, P. F. (1987) Changes in rat plantaris motor unit profiles with advanced age. Mech Ageing Dev, 40, 243-259.

RODRIGUEZ, M. \& TARNOPOLSKY, M. (2003) Patients with dystophinopathy show evidence of increased oxidative stress. Free Radic Biol Med, 34, 1217-1220.

ROUBENOFF, R. (2001) Origins and clinical relevance of sarcopenia. Can J Appl Physiol, 26, 78-89.

ROUBENOFF, R., PARISE, H., PAYETTE, H. A., ABAD, L. W., D'AGOSTINO, R., JACQUES, P. F., WILSON, P. W., DINARELLO, C. A. \& HARRIS, T. B. (2003) Cytokines, insulin-like growth factor 1, sarcopenia, and mortality in very old communitydwelling men and women: the Framingham Heart Study. . Am J Med, 115, 429-435.

SAUGSTAD, O. D. (1996) Role of xanthine oxidase and its inhibitor in hypoxia: reoxygenation injury. Pediatrics, 98, 103-107.

SILVAGNO, F., XIA, H. \& BREDT, D. S. (1996) Neuronal nitric-oxide synthase-mu, an alternatively spliced isoform expressed in differentiated skeletal muscle. J Biol Chem, 271, 11204-11208.

SIU, P. M. \& ALWAY, S. E. (2005) Mitochondria-associated apoptotic signalling in denervated rat skeletal muscle. J Physiol, 565, 309-323. 
SMITH, H. K., PLYLEY, M. J., RODGERS, C. D. \& MCKEE, N. H. (1999) Expression of developmental myosin and morphological characteristics in adult rat skeletal muscle following exercise-induced injury. Eur J Physiol, 80, 84-91.

SOHAL, R. \& WEINDRUCH, R. (1996) Oxidative stress, caloric restriction, and aging. Science, 273, 59-63.

STAMLER, J. S. \& MEISSNER, G. (2001) Physiology of nitric oxide in skeletal muscle. Physiol Rev, 81, 209-237.

TAMAKI, T., AKATSUKA, A., TOKUNAGA, M., ISHIGE, K., UCHIYAMA, S. \& SHIRAISHI, T. (1997) Morphological and biochemical evidence of muscle hyperplasia following weight-lifting exercise in rats. Am J Physiol, 273, 246-256.

TATAR, M., BARTKE, A. \& ANTEBI, A. (2003) The endocrine regulation of aging by insulinlike signals. Science, 299, 1346-51.

TOSCANO, A., MESSINA, S., CAMP, G., DI LEO, R., MUSUMECI, O. \& RODOLICO, C. (2005) Oxidative stress in myotonic dystrophy type 1. Free Radic Res, 39, 771-776.

UCHIYAMA, S., TSUKAMOTO, H., YOSHIMURA, S. \& TAMAKI, T. (2006) Relationship between oxidative stress in muscle tissue and weight-lifting-induced muscle damage. Eur J Physiol, 452, 109-116.

URSO, M. L. \& CLARKSON, P. M. (2003) Oxidative stress, exercise, and antioxidant supplementation. Toxicology, 189, 41-54.

VAN DER MEULEN, J. H., MCARDLE, A., JACKSON, M. J. \& FAULKNER, J. A. (1997) Contraction-induced injury to the extensor digitorum longus muscles of rats: the role of vitamin E. J Appl Physiol, 83, 817-23.

WARNER, H. (1994) Superoxide dismutase, aging, and degenerative disease. Free Radic Biol Med, 17, 249-258.

WEINDRUCH, R. (1995) Interventions based on the possibility that oxidative stress contributes to sarcopenia. J Gerontol A Biol Sci Med Sci, 50, 157-161.

WELLE, S., TOTTERMAN, S. \& THORNTON, C. (1996) Effect of age on muscle hypertrophy induced by resistance training. J Gerontol A Biol Sci Med Sci, 51, 270-275.

XIA, R., WEBB, J. A., GNALL, L. L., CUTLER, K. \& ABRAMSON, J. J. (2003) Skeletal muscle sarcoplasmic reticulum contains a NADH-dependent oxidase that generates superoxide. Am J Physiol Cell Physiol, 285, 215-221. 
ZHANG, L., YU, L. \& YU, C. A. (1998) Generation of superoxide anion by succinatecyctochrome c reductase from bovine heart mitochrondia. J Biol Chem, 273, 3397233976. 